\title{
NANOPARTICLE INCORPORATED POLYMER BRUSH FILMS FOR SENSING INTERFACES
}

FERHAN OKTOBRIADI PUTRA ABDUL RAHIM

School of Chemical and Biomedical

Engineering

A thesis submitted to the Nanyang Technological University in partial fulfilment of the requirement for the degree of

Doctor of Philosophy 
Dedicated to my parents, sister,

my wife and my lovely daughter. 


\section{ACKNOWLEDGEMENTS}

The author would like to express his utmost gratitude to his supervisor Associate Professor Kim Donghwan Richie, for providing guidance, support and imparting valuable knowledge and skills throughout the period of candidature.

He wishes to thank fellow group members Dr. Guo Longhua, Dr. Huang Youju, Dr. Palanisamy Kannan, Dr. Anirban Dandapat, Dr. Chen Hailan and Dr. Si Peng for countless sessions of meaningful discussions as well as for their continual support and encouragement during this period.

He also wishes to thank collaborators Prof. Li Changming, Dr. Donna Zhou, Dr. Fan Hongjin and Dr. Liu Yingshuai, Dr. Wang Yang and Dr. Wu Fang for the sharing of experimental resources and kind assistance. 


\section{ABSTRACT}

The use of polymer brushes has been attracting attention in recent years due to its ability to reduce non-specific adsorption of proteins on a surface. At the same time, noble metal nanoparticles has been widely exploited for chemical and biomolecular sensing applications, particularly via localized surface plasmon resonance and surface enhanced raman spectroscopy. While many conventional plasmonic, nanoparticle solution-based detection methods offer direct observation with high sensitivity and selectivity, they have several limitations. For example, it is difficult to discern color hue or intensity changes in colored samples or complex mixtures such as blood and serum. Nanoparticle in solution are also prone to aggregation over time, reducing shelf life.

This work explores the use of polymer brushes as a matrix for the incorporation of nanoparticles. Sensing interfaces were facilely fabricated by loading unmodified gold nanoparticles (AuNPs) and gold nanorods (AuNRs) into poly(oligo(ethylene glycol) methacrylate) (POEGMA) brushes grown on glass. Depending on their size, the AuNPs would either infiltrate into the brush or adsorb on the brush/solution interface. The attainment of a three-dimensional (3D) assembly of non-infiltrating AuNP was also achieved via a novel process described as in-stacking. The AuNPPOEGMA nanocomposite structure with infiltrated AuNPs was used for lead detection based on lead ion-induced release of thiosulfate-functionalized AuNPs. The decrease in colorimetric intensity over increasing lead concentrations could easily be observed 
via unaided eyes. We obtained a limit-of-detection as low as $25 \mathrm{pM}$ and a dynamic range as wide as 4 orders of magnitude. Lead detection in human serum gave recoveries of $91.0-109.9 \%$, testimony of the effective role of POEGMA as a nonfouling polymer matrix. The AuNP-POEGMA nanostructure with adsorbed AuNPs were employed for the detection of proteins. We found that large plasmonic peak shifts of $\sim 50 \mathrm{~nm}$ were actuated upon in situ functionalization as well as during target detection. They were used for thrombin detection based on aggregation and deaggregation induced upon aptamerization and protein sensing respectively. We obtained a limit-of-detection as low as $0.64 \mathrm{pM}$. Finally, AuNR-POEGMA nanocomposite structures was conveniently achieved by initially studying the influence of ionic strength and surfactant concentration on the assembly of AuNRs on a polystyrene-sulfonate (PSS)-modified substrate. The same principle was eventually applied to obtain tunable assemblies of AuNRs incorporated within the POEGMA. AuNR-POEGMA nanocomposites with low AuNR densities were suitable to be used as LSPR-based sensors while those with high AuNR densities for SERS-based sensors. 


\section{TABLE OF CONTENTS}

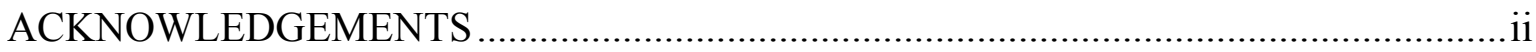

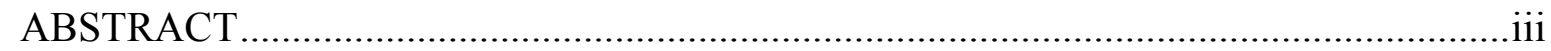

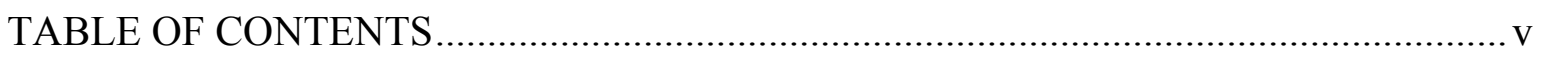

NOTATIONS AND ABBREVIATIONS ..................................................................

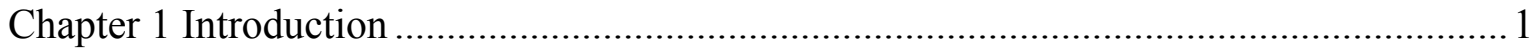

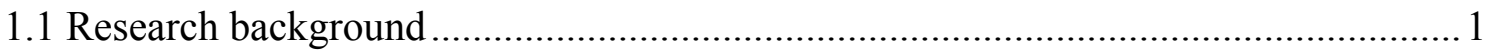

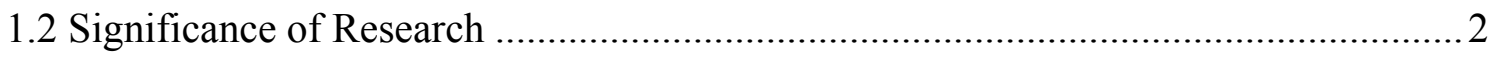

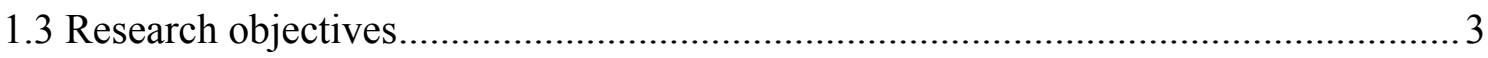

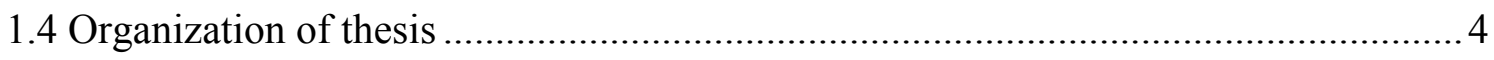

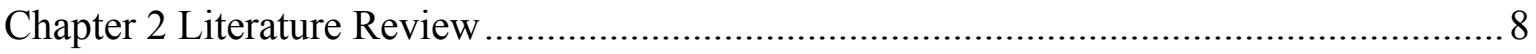

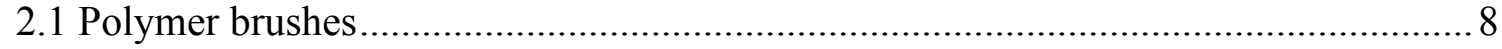

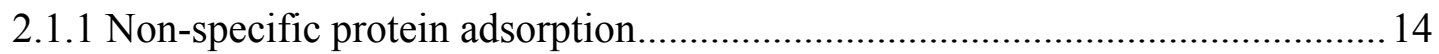

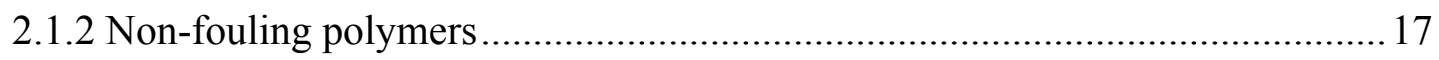

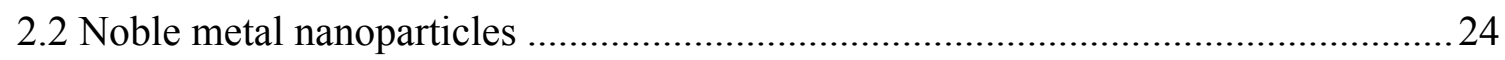

2.3 Polymer brush nanoparticle nanocomposites .......................................................2 29

2.3.1 Polymer brush nanoparticle nanocomposite construction strategies ...............29

2.3.2 Polymer brush nanoparticle nanocomposite applications................................32

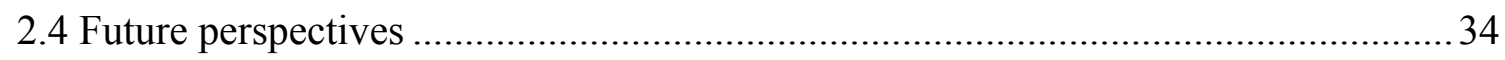

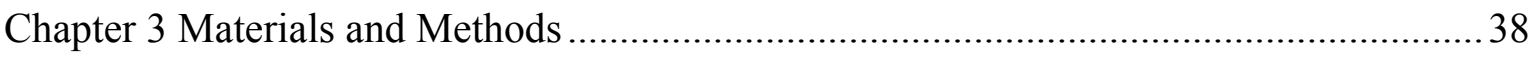


3.1 Common materials

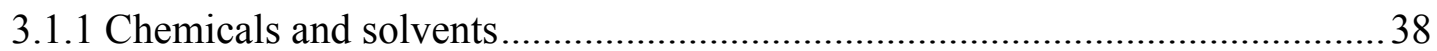

3.1.2 Biomolecules and buffer solutions ........................................................... 38

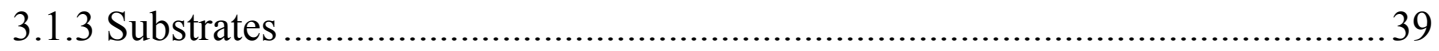

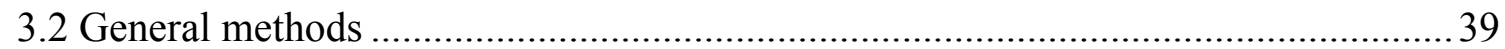

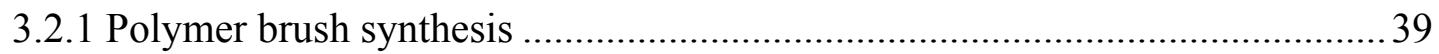

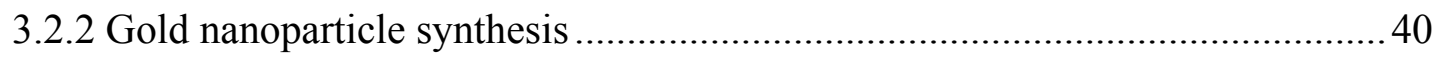

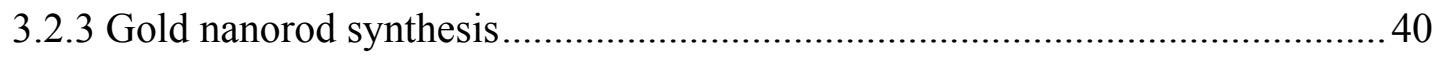

Chapter 4 POEGMA as a Non-Fouling Support Matrix for Ultrasensitive Protein

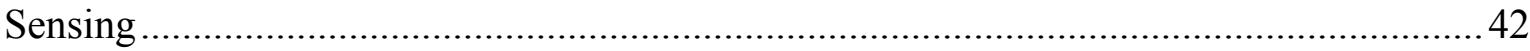

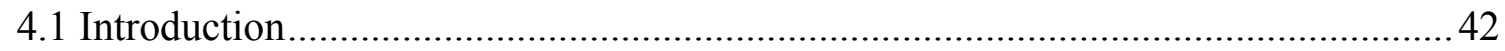

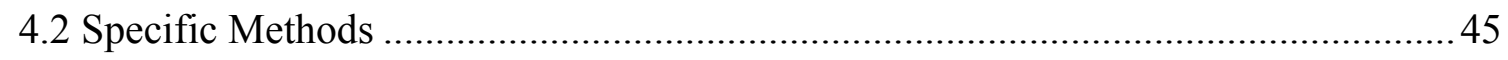

4.2.1 Antibody-spotting for fluorescence measurements .................................... 45

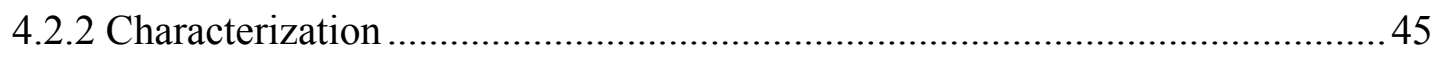

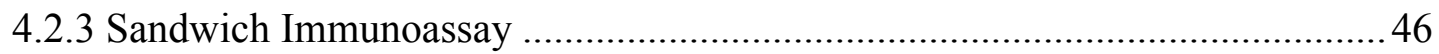

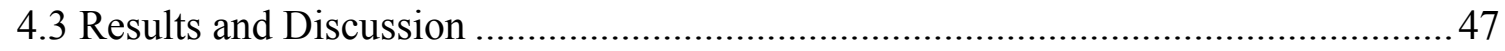

4.3.1 Antibody loading as a function of brush thickness ......................................4

4.3.2 Orientation of physically immobilized antibodies across different brush

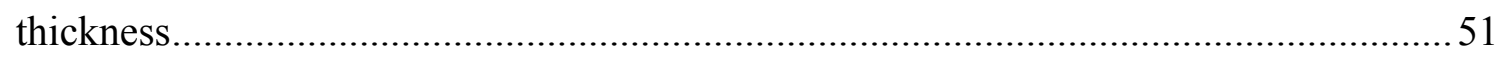

4.3.3 Biomolecular assay based on physically immobilized antibodies...................59

4.3.4 Stages involved in the physical immobilization of antibodies ........................61

4.3.5 Role of simple aggregation in physical immobilization of antibodies .............62

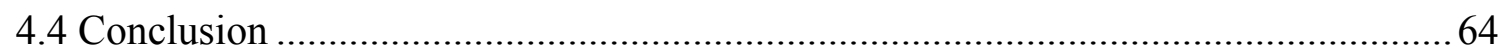


Chapter 5 Gold Nanoparticle Incorporation in POEGMA via Infiltration and Its Application in Solid-Phase Colorimetric Sensing of Lead

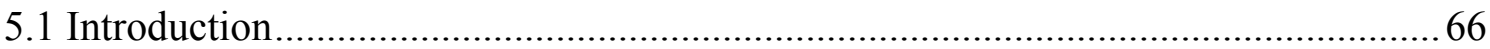

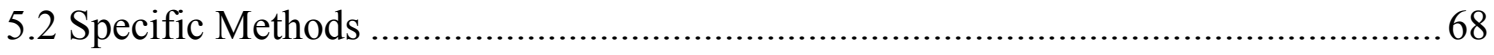

5.2.1 Nanoparticle incorporation into POEGMA …................................................6

5.2.2 Nanoparticle postfunctionalization and lead detection ..................................68

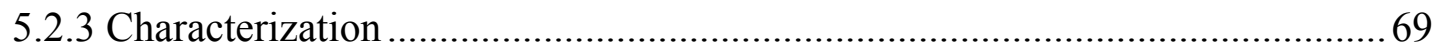

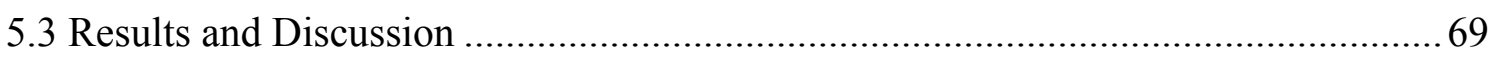

5.3.1 Gold nanoparticle loading as function of polymer brush thickness..................69

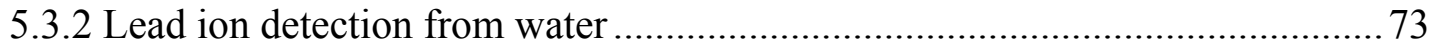

5.3.3 Effect of brush thickness on detection performance..................................... 81

5.3.4 Selectivity of the nanocomposite detection platform .................................. 84

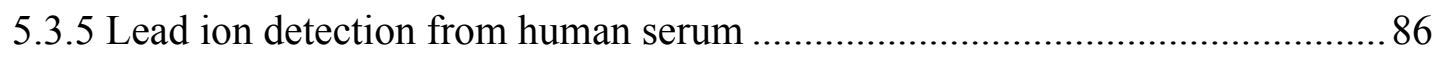

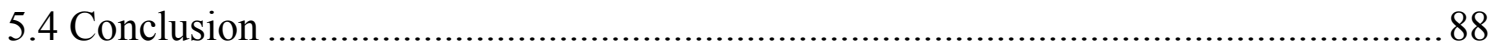

Chapter 6 Three-Dimensional Gold Nanoparticle Incorporation in POEGMA via In-

Stacking and Adsorption on Dual Round Growth of POEGMA for Plasmonic Sensing

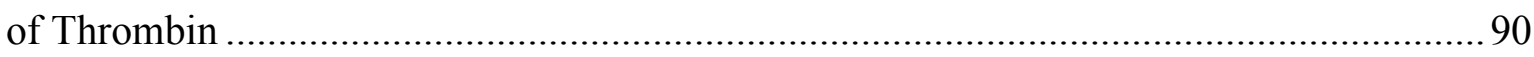

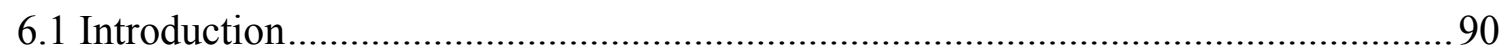

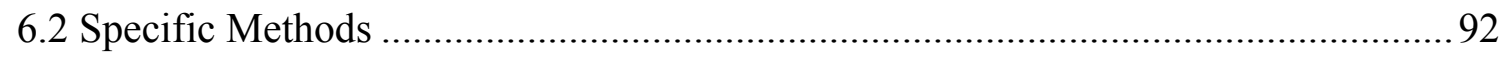

6.2.1 Gold nanoparticle incorporation via in-stacking ........................................ 92

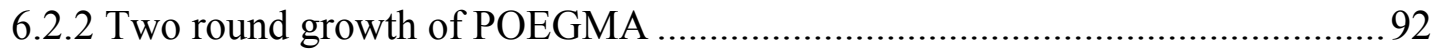

6.2.3 Gold nanoparticle incorporation on POEGMA grown for two rounds ............ 92

6.2.4 Gold nanoparticle postfunctionalization and thrombin detection................... 92

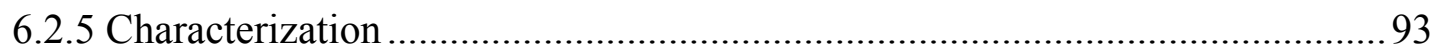


6.3.1 In-stacking of nanoparticles in brushes of different thicknesses

6.3.2 Effect of different immersion times per cycle

6.3.3 Mechanism behind in-stacking .....

6.3.4 Feasibility of in-stacking nanoparticles of different sizes

6.3.5 Rough surface POEGMA based on dual rounds of brush synthesis.

6.3.6 Nanoparticle incorporation on rough surface POEGMA

6.3.7 Thrombin sensing based on nanoparticle mobility on rough surface

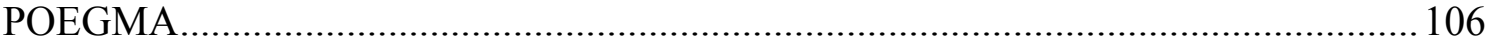

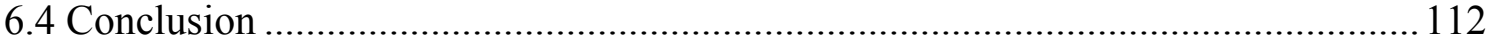

Chapter 7 Effects of Ionic Strength and Surfactant Concentration on Surface Adsorption of Gold Nanorods

7.1 Introduction

7.2 Specific Methods

7.2.1 Formation of APTS-PSS layer.

7.2.2 Surface assembly of gold nanorods

7.2.3 Gold nanorod surface assembly retention study

7.2.4 Characterization

7.3 Results and Discussion

7.3.1 Two-tiered shielding effect and general parabolic trend in surface density ... 123

7.3.2 Charge coverage around gold nanorods from peak $\mathrm{NaCl}$ concentration onwards

7.3.3 Modeling second shielding layer as an isolated doble layer.

7.3.4 Trend variation at high $\mathrm{CTAB}$ concentration 
7.3.5 Trend variation at low $\mathrm{CTAB}$ concentration

7.3.6 Nature of CTAB in second shielding layer.

7.3.7 Nanorod-substrate interactions

7.3.8 Robustness of electrostatic nanorod surface assembly

7.4 Conclusion

Chapter 8 Gold Nanorod Adsorption on POEGMA and Its Application in Surface

Enhanced Raman Spectroscopy

8.1 Introduction

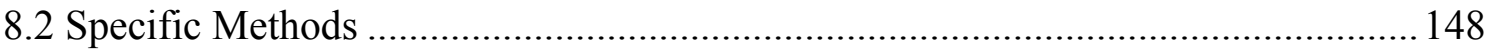

8.2.1 Gold nanorod self-assembly into floating super-aggregates........................ 148

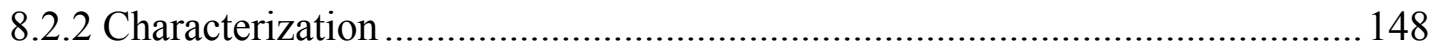

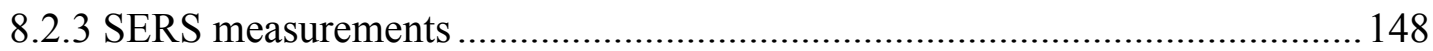

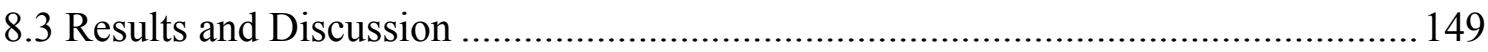

8.3.1 Significance of ionic strength adjustment for nanorod adsorption on POEGMA.

8.3.2 Effect of brush thickness on gold nanorod assembly 152

8.3.3 Uniformity of gold nanorod assembly over large areas 159

8.3.4 Three-dimensional assembly of gold nanorods in POEGMA 160

8.3.5 SERS enhancement capability of gold nanorod-incorporated POEGMA ...... 165

8.4 Conclusion 170

Chapter 9 Conclusion and Future Direction

9.1 Conclusion

9.2 Future works

Bibliography 176 
List of Publications

Appendix.

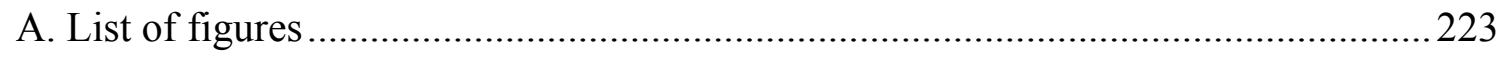

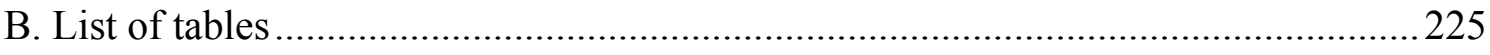

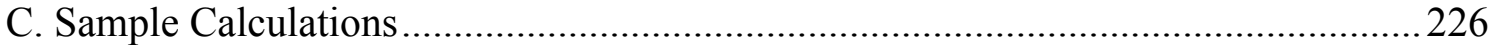


NOTATIONS AND ABBREVIATIONS

\begin{tabular}{|c|c|}
\hline Notation/Abbreviation & Name \\
\hline $2 \mathrm{ME}$ & 2-mercaptoethanol \\
\hline AFM & Atomic force microscopy \\
\hline APTS & Aminopropyltriethoxysilane \\
\hline ATRP & Atomic transfer radical polymerization \\
\hline AuNP & Gold nanoparticle \\
\hline AuNR & Gold nanorod \\
\hline CTAB & Cetyltrimethylammonium bromide \\
\hline DLS & Dynamic light scattering \\
\hline FESEM & Field-emission scanning electron microscopy \\
\hline IgG & Immunoglobulin $\mathrm{G}$ \\
\hline LOD & Limit of detection \\
\hline LOL & Limit of linearity \\
\hline LSPR & Localized surface plasmon resonance \\
\hline POEGMA & Poly(oligo ethylene glycol) methacrylate \\
\hline R6G & Rhodamine $6 \mathrm{G}$ \\
\hline RMS & Root-mean-square \\
\hline $\mathrm{S} / \mathrm{N}$ & Signal-to-noise ratio \\
\hline SI-ATRP & Surface-initiated atomic transfer radical polymerization \\
\hline SPR & Surface plasmon resonance \\
\hline TBA & Thrombin-binding aptamer \\
\hline TM-AFM & Tapping-mode atomic force microscopy \\
\hline XPS & X-ray photoelectron spectroscopy \\
\hline
\end{tabular}




\section{Chapter 1 Introduction}

\subsection{Research background}

Over the past two to three decades, there have been tremendous advances in the development of biosensing platforms capable of quantifying a wide range of analytes which have clinical, medical, biotechnological as well as environmental significance. Such multidisciplinary nature of biosensing promotes the rapid expansion of its research and development.

The development of a vast array of biosensing methods has been possible owing to the strong recognition capability of biological systems via specific binding interactions. However, biomolecules also have a strong tendency to physically adsorb onto a solid substrate in the absence of specific receptor-recognition interactions. This is referred as non-specific adsorption. Such adsorption typically reduces the effectiveness and reliability of a biosensing interface by introducing undesirable features such as huge background noise and false positives. Conversely, this leads to a sharp decline in biosensor accuracy and sensitivity, constituting the bottleneck for further development of biosensors toward practical applications. In order to create worthy materials for a wide range of biosensing applications, it is a requirement for interfaces to possess or induce both enhanced specific binding and reduced nonspecific binding.

The elimination of non-specific adsorption and improvement in signal-to-noise ratio can lower detection limits by up to a few orders of magnitudes. In an attempt to achieve this, the synthesis of polymer brushes which have supreme non-fouling 
capacities has received significant attention over the recent years. At the same time, various plasmonic nanostructures have been utilized to enhance signals arising from the specific binding event. Therefore, the integration of both classes of materials is expected to have significant impact on the overall development of effective biosensing devices with lower limits of detection. This would enable the early detection of diseases which are currently not achievable due to the low threshold limit of their biomarkers.

\subsection{Significance of research}

There is plenty of room for the development of polymer brush nanoparticle nanocomposites. For most polymer brushes which are capable of preventing nonspecific adsorption, focus has most often been one-dimensional, mainly emphasizing its non-fouling property. The feasibility for it to serve as an efficient support matrix for nanoparticles has rarely been explored. This is primarily due to the fact that polymer brushes are usually grown at high grafting densities, hampering nanoparticle infiltration. Also, chemical modifications which, on one hand, promote nanoparticle loading, may compromise the non-fouling effectiveness of the polymer brush.

It has been recently reported that polyethylene glycol groups can form a pseudoether crown structure which have an affinity for gold surfaces via a multidentate interaction. This may pave the way for polymer brushes which contain ethylene glycol groups either in their main or side branches to serve as a matrix for the incorporation of gold nanoparticles. In order to form nanocomposites with a three-dimensionally assembled high loading of nanoparticles, an alternative method to simple infiltration needs to be developed to overcome the barrier associated with high grafting densities. 
The achievement of such polymer brush nanoparticle nanocomposites can ultimately serve as interesting sensing platforms such as solid-phase colorimetric sensors which rely on the nanomobility of the nanoparticles within the polymer brush matrix. The detection scheme can also be transferred to the electrochemical setting where such composites can be obtained on electrode surfaces.

\subsection{Research objectives}

The primary research objective is to obtain novel chemical and biomolecular sensing platforms through the incorporation of gold nanoparticles in polymer brush, with a central emphasis on achieving better sensing performances by means of a simple and straightforward approach. This objective will be achieved through several specific aims.

This includes exploring and establishing new immobilization techniques, which reduce if not eliminate the need for modification to both the brush and the nanoparticles, allowing them to retain their characteristic properties. Such techniques should also be universal and applicable to nanoparticles of different sizes, enabling them to be assembled in a controlled and predictable manner. In relation to this aim, the three-dimensional matrix of the brush would also be exploited to attain highdensity three-dimensional assemblies of gold nanoparticles.

Once immobilization techniques have been established, the next step would be to design sensing schemes that maximize signals arising from the localized surface plasmon resonance (LSPR) phenomenon either through increasing peak shifts in the absorption spectra or through intensifying surface enhanced Raman spectroscopy (SERS) signals. While intensifying signals from SERS is relatively straightforward 
and, in most cases, solely rely on the arrangement and orientation of nanoparticles on the substrate, increasing LSPR peak shifts can be achieved through many ways. One of the most effective ways is through nanoparticle aggregation. However, while targetinduced aggregation is easily achieved in the solution phase, it has not been achieved in the solid phase, simply due to the inability of nanoparticles to move around once they are immobilized on a substrate. Therefore, the aim at this stage is to explore the nanoparticle mobility within the brush. This will be divided into two parts. The first part would demonstrate target-induced release of nanoparticles from the brush while the second part would demonstrate target-induced rearrangement of nanoparticles on the brush, without significant loss to the bulk medium.

\subsection{Organization of thesis}

The primary objective is achieved through several sequential works performed to meet the specific aims and of which the findings have been documented in dedicated chapters in this thesis. This thesis is organized as follows.

Chapter 1, 'Introduction' presents the background of the research field and outlines the significance and objectives of the $\mathrm{PhD}$ research.

Chapter 2, 'Literature Review' discusses the recent advances in polymer brush synthesis and applications, nanoparticle synthesis and applications as well as current methodologies for nanocomposite construction. Early works on non-specific adsorption of proteins on surfaces as well as various methods for protein repulsion is presented before discussing the application of polymer brushes as non-biofouling coatings. The future perspectives with respect to the application of polymer brush nanoparticle nanocomposites are also briefly discussed. 
Chapter 3, 'Research Methodology' lists the common experimental chemicals and materials as well as protocols used throughout the study.

Chapter 4, 'POEGMA as a Non-Fouling Support Matrix for Ultrasensitive Protein Sensing' establishes the non-fouling property of POEGMA by means of demonstrating low limits of IgG detection using antibodies physically immobilized onto the brush. This chapter studies the arrangement of Anti-IgG antibodies as a consequence of varying polymer brush thickness and particularly highlights the feasibility of foreign body infiltration and embedment within the depth of the brush. This chapter serves as the precursor for following chapters, which explores the feasibility of nanoparticle incorporation.

Chapter 5, 'Gold Nanoparticle Incorporation in POEGMA via Infiltration and Its Application in Solid-Phase Colorimetric Sensing of Lead' explores the incorporation of as-prepared small gold nanoparticles $(<5 \mathrm{~nm}$ in diameter) within the polymer brush grown on a glass substrate and demonstrates the feasibility of nanoparticle postfunctionalization within the brush matrix. The functionality of the entire nanocomposite as a new type of solid-phase colorimetric chemical sensor is also proven based on lead ion-triggered release of functionalized gold nanoparticles, leading to a decrease in colorimetric intensity. This chapter highlights the convenience of gold nanoparticle incorporation in POEGMA and, in particular, the ability of the nanoparticles to be controllably released. This hinted the prospect of developing novel solid-phase colorimetric biosensors based on nanoparticle mobility on polymer brushes.

Chapter 6, 'Three-Dimensional Gold Nanoparticle Incorporation in POEGMA via In-Stacking and Adsorption on Dual Round Growth of POEGMA for Plasmonic 
Sensing of Thrombin' further explores the feasibility of incorporating larger sizes of gold nanoparticles ( $>10 \mathrm{~nm}$ in diameter) within the polymer brush matrix. A novel methodology for the formation of three-dimensional nanoparticle assembly in the polymer brush is presented. By employing the characteristic property of the brush, which swells in solution and collapses when dried, it is shown that repeated drying and immersions in gold nanoparticle solution can easily increase nanoparticle loading in a stacked fashion without the risk of nanoparticle aggregation. Alternatively, growing POEGMA in successions to present a rough surface morphology can also reduce the risk of aggregation. In the case of the latter, it is also shown that nanoparticles can be mobilized when they are post-functionalized and employed to detect a protein of interest. This chapter highlights the feasibility of transferring a solution-phase colorimetric platform to the solid-phase, particularly with respect to common sensing pathways based on nanoparticle aggregation and de-aggregation.

Chapter 7, 'Effects of Ionic Strength and Surfactant Concentration on Surface Adsorption of Gold Nanorods' presents a detailed fundamental study on the combined consequence of adjusting the ionic strength and surfactant concentration on gold nanorod solutions on its assembly on a treated surface. The highlight of this chapter is the establishment of the 'double-shielding effect' around gold nanorods, which explains the ability for salt ions and surfactant molecules to surround the gold nanorod in an alternating manner, resulting in gradual de-stabilization and re-stabilization. Based on this, assembly of as-prepared gold nanorods on a surface can be conveniently tuned simply through careful adjustment of ionic strength, without the need for any post-functionalization procedures as commonly performed. This work serves as the primary basis for the proceeding chapter. 
Chapter 8, 'Gold Nanorod Adsorption on POEGMA and Its Application in Surface Enhanced Raman Spectroscopy' serves to prove the feasibility of anisotropic nanoparticle immobilization. Gold nanorods can be controllably incorporated with POEGMA to form two-dimensional assemblies as well as long-range threedimensional structures referred to as floating super-aggregates. In the case of the latter, the depth of the brush allows the gold nanorods to be embedded with different orientations. More importantly, such assemblies can be uniformly obtained over large surfaces, allowing them to be used as SERS active substrates.

Chapter 9, 'Conclusions \& Future Directions' summarizes the main findings from Chapters 4 to 8 of this work. Several possible future works are also proposed. 


\section{Chapter 2}

\section{Literature Review}

In the development of new materials, the material can be broken down into the bulk phase and the surface. The ability to provide novel controllable surface topography with the ability to tune surface properties represents an interesting prospect since it enables one to retain the properties of the bulk material while changing the property at the interface. This is performed to improve the performance of the material and to introduce new physical, chemical as well as biological functionalities to the surface.

\subsection{Polymer brushes}

In order to achieve this, the use of polymer brushes has recently gained attention ${ }^{1-}$ ${ }^{3}$, amongst other types of surface coatings such as spin-coated bulk polymer coatings ${ }^{4}$ and layer-by-layer assemblies ${ }^{5}$. Polymer brushes are polymer chains or macromolecular moeities with one end tethered to the surface with the length of the polymer chain being at least twice the grafting distance ${ }^{6}$. Due to this nature, there will be a huge amount of steric repulsion, causing the polymer chains to swell in a favorable solvent. This effectively creates a functional film with considerable thickness. In addition, the access to a vast range of functional groups also allows researchers to easily control the surface properties. Following the rapid growth of the understanding and use of polymer brushes, as well as the establishment of several synthesis routes, many researchers have since exploited the properties of polymer brushes and used them for various applications. 

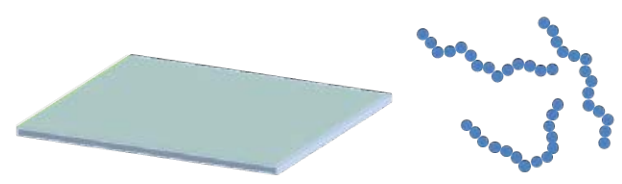

Pre-synthesized

polymer brush

Polymer-attracting substrate
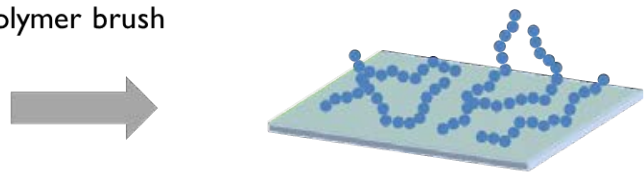

\section{Grafting-to}
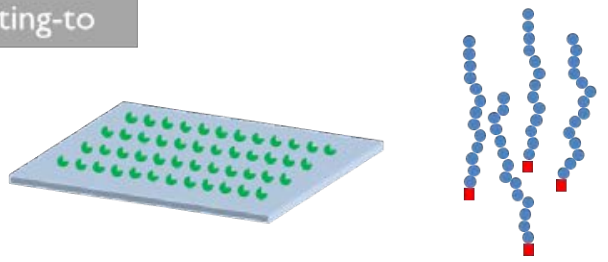

Pre-synthesized

polymer brush

terminated with reactive end-group

Reactive surface group-modified substrate
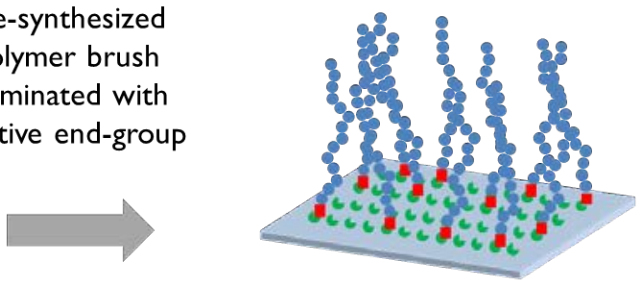

\section{Grafting-from}
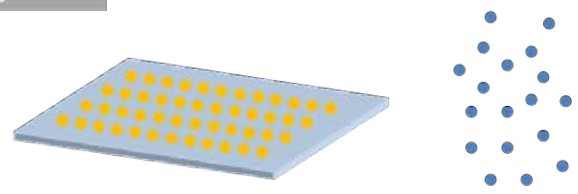

Monomer

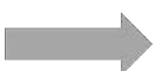

Initiator-modified substrate

Figure 2-1. A summary of the three main techniques for the attainment of polymer brushes on a substrate.

Aside from simple adsorption, polymer brushes can be attached to substrate surfaces based on either the 'grafting to' or the 'grafting from' approach ${ }^{7}$ (Figure 2-1). The 'grafting to' method relies on pre-synthesized polymers which are attached to the surface either via physical or chemical adsorption. On the other hand, the 'grafting from' method relies on controlled polymerization techniques that allow accurate control over brush thickness, composition, and architecture. Among the commonly used methods are anionic, cationic, ring-opening as well as ring-opening methathesis polymerizations. However, the current most frequently used method for the synthesis of brushes via the grafting from approach relies on surface-initiated controlled radical 
polymerization (SI-CRP) techniques. And among the numerous SI-CRP techniques, atomic transfer radical polymerization (ATRP) has been most-commonly used since it is the most robust ${ }^{8}$. Briefly, ATRP is based on the reversible redox activation of a dormant alkyl halide-terminated polymer chain end by a halogen transfer to a transition metal complex. The formal homolytic cleavage of the carbon-halogen bond, which results from this process, generates a free and active carbon-centered radical species at the polymer chain end. This activation step is based on a single electron transfer from the transition metal complex to the halogen atom, which leads to the oxidation of transition metal complex. Then, in a fast, reversible reaction, the oxidized form of the catalyst reconverts the propagating radical chain end to the corresponding halogen capped dormant species. These processes are summarized in Figure 2-2. Many parameters, such as ligand to transition metal ratio, $\mathrm{Cu}$ (II) to $\mathrm{Cu}$ (I) ratio, type of ligand, counterion, solvent, or initiator, influence the performance of ATRP and thus offer the possibility to fine-tune the reaction.

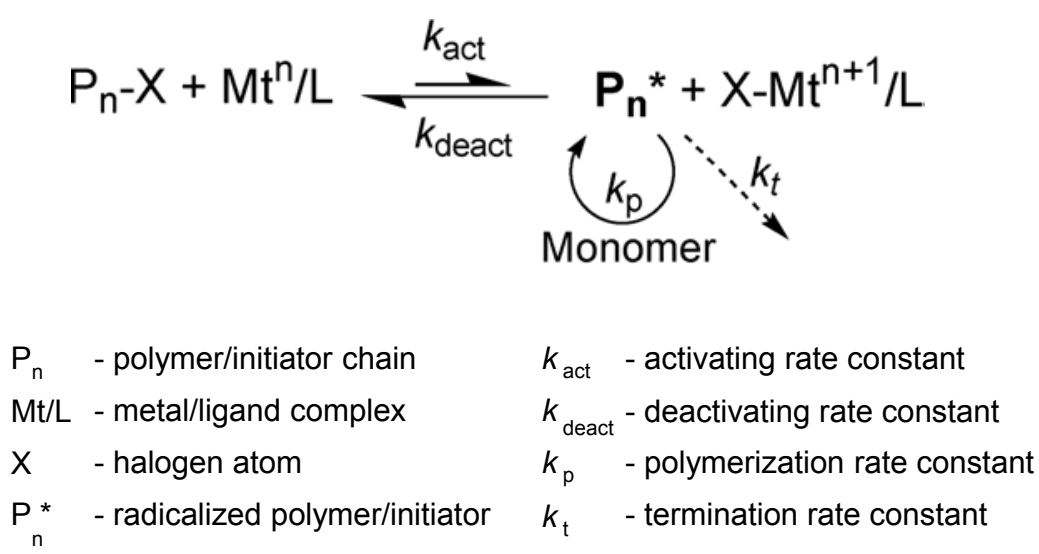

Figure 2-2. A summary of processes involved in atomic transfer radical polymerization (ATRP). 
An extension to the ATRP technique is the surface-initiated ATRP (SI-ATRP), which was first reported by Huang and Wirth, through the grafting of poly(acrylamide) (PAM) brushes from benzylchloride-derivatized silica particles ${ }^{9}$. In SI-ATRP, chain growth starts from a surface-immobilized ATRP initiator. The same transition metal complexes that mediate SI-ATRP can also be applied to grow polymer brushes in a controlled fashion from surfaces modified with a conventional radical initiator. Since its founding, SI-ATRP has clearly proven to be an excellent technique to prepare polymer brushes. One of the most celebrated aspect of ATRP is its chemical versatility and robustness. ATRP can be used with a large assortment of monomers and functional groups, and tolerates a relatively high degree of impurities. Specifically, ATRP is relatively insensitive toward small residual oxygen, which are readily removed by oxidation of the ATRP catalyst. Since most of the standard ATRP catalyst systems, as well as surface immobilizable initiators are commercially available or can be can be conveniently synthesized makes ATRP an attractive technique from an experimental point of view.

The ability to predictably control the properties of the brushes grown via controlled radical polymerization techniques permits the innovation of novel polymeric architectures since these polymer brushes can be grown with precise grafting densities $(\sigma)$ and thicknesses, which correspond to the averaged lengths of the polymer chains $(h)$. These two properties represent the most critical parameters that govern the morphology of the polymer brush. When the inter-grafting distance $(D)$ is sufficiently high (i.e. low $\sigma)$, such that it is more than twice the radius of gyration $\left(R_{\mathrm{g}}\right)$ of the polymer chain, the polymer chains adopt a mushroom-like configuration. As $D$ decreases less than twice $R_{\mathrm{g}}$, the polymer chains begin to stretch into a brush-like configuration. The chain stretching is related to the grafting density through the 
scaling law stating that $\mathrm{h}$ is proportional to $N \sigma^{\mathrm{v}}$, where $\mathrm{N}$ is the degree of polymerization and $\mathrm{v}$ is the exponent, in the range of 0 to $1^{10,11}$. The role of the critical parameters in determining the morphology of the brush is illustrated in Figure 2-3.

Furthermore, the use of controlled radical polymerization techniques also allows for the ease of copolymerization. Aside from the architectural perspective (i.e. the ability for the polymer brushes to be cross-linked etc), this also allows for the incorporation and integration of various functionalities. Such functionalities allow surfaces that have been treated with polymer brushes to be used in a variety of applications. 


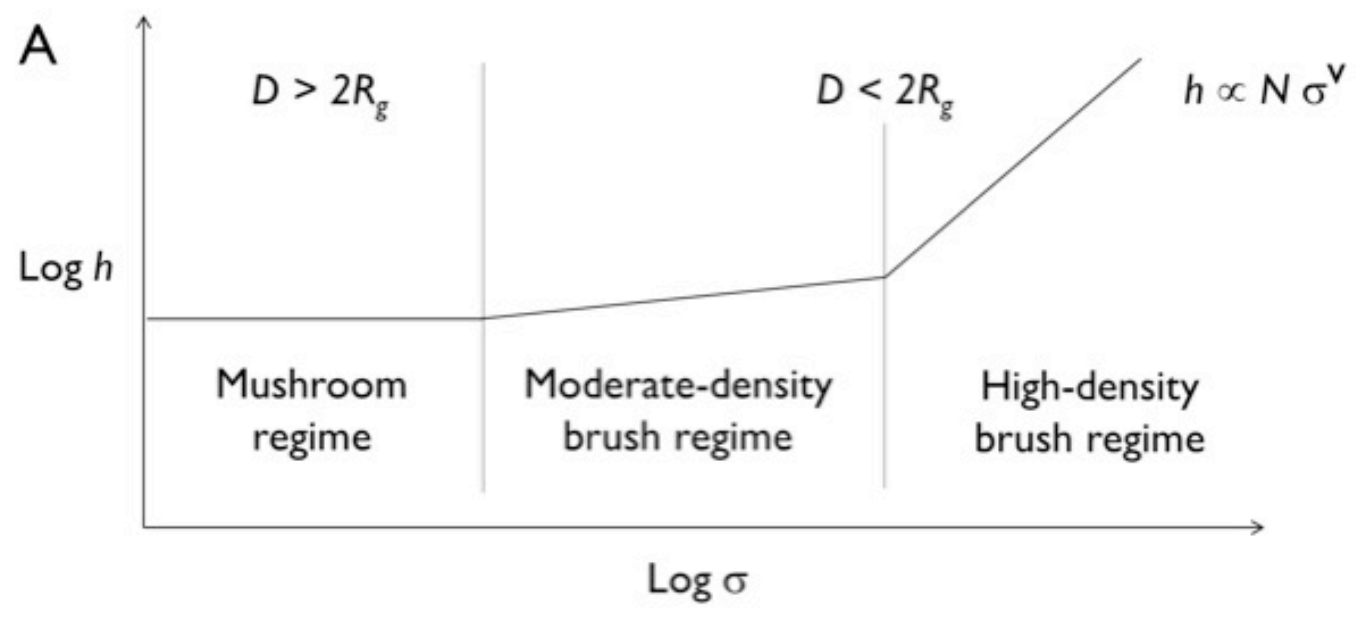

B

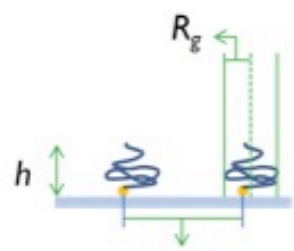

$D$

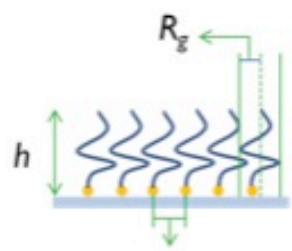

D

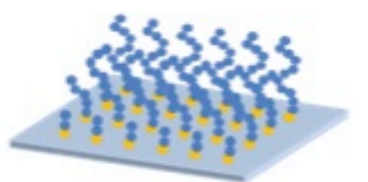

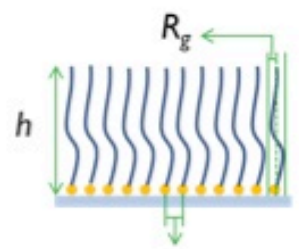

D

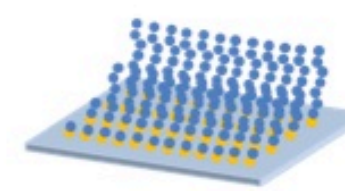

Figure 2-3. A summary of the critical parameters and the types of brush formed. (A) Correlation between the average brush thickness $(h)$ and grafting density $(\sigma)$, (B) Schematic representation of the average brush thickness $(h)$, grafting density $(\sigma)$ and radius of gyration $\left(R_{\mathrm{g}}\right)$ across the different brush regimes. (C) Schematic illustration of brush growth with respect to time $(t)$.

Ever since the founding of polymer brushes and the ability to create well-defined structures, there have been an increasing number of applications associated with surfaces treated with polymer brushes ${ }^{12}$. In a wide variety of applications, the two most prominent qualities exploited from polymer brushes are its stimuli responsiveness as well as its non-fouling property ${ }^{13}$. However, the application that is 
most relevant to this thesis is the non-fouling property associated with polymer brushes.

\subsubsection{Non-specific Protein Adsorption}

At this juncture, it is important to highlight the significance of non-specific protein adsorption to solid surfaces. In general, any artificial material that is exposed to a complex biological solution will be prone to non-specific protein adsorption. This can lead to adverse effects including thrombosis ${ }^{14-16}$ (local blood coagulation or clotting) and bacterial adhesion ${ }^{17-19}$, jeopardizing the functionality and effectiveness of prosthetic devices and implants ${ }^{20-22}$. The mechanism for such an occurrence has been a topic for in-depth discussion amongst established researchers, especially in the 1980s and 1990s. For example, Willem Norde and co-workers had done several works ${ }^{23-27}$ to characterize the binding of proteins to a surface, describing how proteins can undergo structural rearrangements upon attachment to the surface ${ }^{25,27}$, leading to dehydration of the sorbent surface and redistribution of charged groups. Wertz, Santore and coworkers also studied, through a series of experimental works, the adsorption, reorientation and interfacial relaxation kinetics of proteins on hydrophobic and hydrophilic surfaces ${ }^{28-32}$. Lyklema and co-workers used Immunoglobulin G (IgG) in particular to study protein adsorption dynamics ${ }^{33-35}$. They described the interfacial behavour of $\mathrm{IgG}$ upon adsorption ${ }^{35}$ and how it can change its conformation and configuration when its surface concentration is increased ${ }^{34,35}$. They further described how surface adsorption affects the binding affinity for antigens ${ }^{36}$. In a separate series of works, they studied protein adsorption a different approach, by treating proteins as colloidal systems ${ }^{37,38}$.

Adsorption on specific types of material was also the focus of some groups. For example, early works by Lyman and co-workers investigated protein adsorption on 
synthetic polymer surfaces and studied the correlation between the surface properties, chemical structure and morphologies of the polymer to the amount of proteins (e.g. albumin and fibrinogen) adsorbed ${ }^{39-41}$. In addition, van Wachem, Beugeling and coworkers also investigated the adhesion of cultured human endothelial cells on synthetic polymer surfaces as a consequence different wettabilities ${ }^{42,43}$ and protein adsorption $^{44}$. The effect of cell adhesion in promoting biocompatibility was also mentioned by Hoffmann et. al. whereby they had earlier found that the pre-adsorption of albumin, $\gamma$-globulin and fibrinogen on poly(2-hydroxyethyl methacrylate) and poly(N-vinyl-2-pyrrolidone) surfaces was shown to significantly affect the adhesion of cells ${ }^{45}$.

Besides understanding the mechanism behind how proteins adsorb to surfaces, preferential adsorption as well as the order in which they adsorb was also investigated. Leo Vroman and co-workers, who deal with the interaction of blood proteins with interfaces, described protein exchange upon adsorption from blood and formulated what is known as the 'Vroman effect' whereby low molecular weight proteins adsorb first before being displaced by the later arriving higher molecular weight proteins ${ }^{46}$. Ellipsometry, a non-destructive optical characterization technique ${ }^{47,48}$, was employed to study rapid exchanges of plasma proteins at the liquid/solid interface ${ }^{49}$. They also described how protein from plasma adsorbs on spaces with different geometries such as narrow flow channels ${ }^{50,51}$, as well as how protein adsorption can affect the wettability of surfaces ${ }^{52}$. The group of Erwin Vogler presented a comprehensive series of works published under an overarching theme referred as 'volumetric interpretation of protein adsorption ${ }^{53-58}$. Besides topics relating to competitive adsorption from mixtures and Vroman effect ${ }^{53,56}$, subjects covered under the theme included those related to mass and energy balance ${ }^{55}$ as well as physical chemistry parameters such 
interphase volume and free energies of adsorption ${ }^{54,57,58}$. With particular attention on implants and prosthetics, Baier and co-workers described several physical parameters that can either lead to or prevent bioadhesion ${ }^{59,60}$. They have also reviewed the conditioning of surfaces to suit the biomedical environment ${ }^{61}$, and studied non-specific protein adsorption particularly involving dental and medical implant technologies ${ }^{62,63}$.

Besides affecting prosthetics and implants, non-specific adsorption of proteins also affects the performance of biosensors. The adventitious binding of background proteins could block reactive sites and prevent target proteins from being detected by the recognition elements. This will degrade the sensors' overall detection sensitivity and specificity. There exists several works in literature that studied non-specific binding in relation to biosensing. This was made possible especially with the establishment of the surface plasmon resonance (SPR) technique, first reported by Liedberg et.al. for the purpose of gas detection and biosensing ${ }^{64}$. While SPR was later utilized for the determination of target proteins, it was initially used to study protein binding to a surface per se, without any form of specificity. The experimental setup for SPR was also described by Jonsson et. al., consisting of a sensor chip interface made with a thin layer of gold deposited on glass ${ }^{65}$. The binding of proteins to this interface will cause a red shift in the SPR signal. This red shift could in turn be correlated to the amount of proteins adsorbed on the surface. Interestingly, this technique also allows protein binding to be observed in real-time, making it possible to monitor multimolecular complexes as they form. The method for correlating the absolute surface concentration of surface bound proteins to the surface plasmon resonance is also detailed in a separate work by Stenberg et. al., by using radiolabeled proteins that adsorb on dextran hydrogel on a sensor chip ${ }^{66}$. Optical models were then developed to describe the correlation between the SPR responses to the distribution of protein 
within the hydrogel volume. Later, for the purpose of biospecific interactions and biosensing, the group of Lofas et. al. described a method for covalent immobilization of ligands or recognition elements within carboxymethylated dextran hydrogel matrix $^{67,68}$. This increased the immobilization capacity compared to monolayer-based coatings. The principles and applications of SPR biosensing based on an extended interaction matrix was further described and summarized by Liedberg et. al. through a detailed review paper ${ }^{69}$.

\subsubsection{Non-fouling polymers}

Before the advent of polymer brush, one of the ways to reduce non-specific adsorption of proteins was via surface passivation or pre-coating with albumin. In contrast, there are also proteins such as fibrinogen, which when pre-coated on a surface, has an opposite effect and could promote thrombosis. A study by Park et. al. found that when dimethyldichlorosilane-coated glass was coated with albumin, platelets could not spread on the surface ${ }^{70}$. Only when fibrinogen is gradually introduced could the platelets be activated. Interestingly, they found that even when the surface is dominated by albumin, the platelets could still be fully activated as long as there is sufficient amount of fibrinogen on the surface (i.e. minimum surface concentration is reached). Therefore, pre-coating with albumin does not represent a full-proof approach to achieve non-fouling behaviour, which eventually led to the exploration of polymer coatings.

Even then, the use of polymers to prevent non-specific adsorption began with selfassembled monolayers. A study was conducted by Harder et. al. to show how molecular conformation of oligo(ethylene glycol)-terminated self-assembled monolayers can contribute to non-fouling properties on the surface ${ }^{71}$. They characterized the monolayers using infrared adsorption (FTIR) and X-ray 
photoelectron spectroscopies and found that crystalline helical and amorphous forms of oligo(ethylene glycol) on gold substrates is most effective in preventing nonspecific protein adsorption. Tengvall and co-workers studied the adsorption of plasma proteins on functionalized self-assembled monolayers ${ }^{72,73}$. In a separate study, the adsorption of fibrinogen, serum and plasma on well-defined oligo(ethylene glycol)terminated alkane thiols was investigated by Benesch et. al. using ellipsometry ${ }^{74}$. Hoffmann and co-workers studied the effect of attaching poly(ethylene oxide) (PEO) groups to poly(ethylene terephthalate)/allylamine (PET/ALAM) coatings and found that PEO significantly reduced fibrinogen adsorption ${ }^{75}$ (Figure 2-4). On the other hand, Prime et. al. studied the adsorption of fibrinogen, lysozyme, pyruvate kinase and RNAase A on end-attached oligo(ethyelene oxide) self-assembled monolayers ${ }^{76}$. They concluded that resistance to protein adsorption increased with longer chains of oligo(ethylene oxide). Otsuni et. al. utilized the SPR technique to study self-assembled monolayers attached with a range of different polymeric functional groups and concluded that those that resisted protein adsorption were generally hydrophilic, contained groups that were hydrogen-bond acceptors but not hydrogen-bond donors and electrically neutral ${ }^{77}$. Whitesides and co-workers also showed, through the use of SPR, a comparison of the effectiveness of different polymeric thin films containing oligo(ethylene oxide) derivates in preventing non-specific protein adsorption ${ }^{78}$ (Figure 2-5).

Among the earliest polymer brushes recognized as an excellent candidate to invoke non-fouling behaviour came in the form of polyethylene glycol (PEG) grafted to surfaces. In fact, PEG has become widely known for this property and the Food and Drug Administration (FDA) approves PEG-based materials for use in clinical applications. Even In the early years, many detailed investigations, both theoretically 
and experimentally, have been conducted to study the parameters affecting its nonfouling behaviour. This includes the work by Jeon et. al. which theoretically studied the event of protein of infinite size approaching PEG brush chains grafted to a hydrophobic surface. ${ }^{79}$ In their work, three forces had been taken into account, namely attractive hydrophobic interaction between the solid surface and the protein (via hydrophobic patches), van der Waals attraction as well as the competing steric repulsion from the brush. In studies conducted by de Gennes ${ }^{80,81}$, steric repulsion can be briefly described as a consequence of osmotic pressure and elastic restoring forces. The study by Jeon et. al. concluded that the excellent non-fouling property of PEG arises when van der Waals interaction with the protein becomes negligible as steric repulsion dominates; consequently this meant that high surface density and long chain length are desirable for protein resistance. They also found that surface density was a greater contributor to non-fouling behaviour than chain length. This work is further supported by McPherson et. al., who claimed, through calculating attractive and repulsive interaction between lysozyme and the PEG-grafted surface, that for steric repulsion to be effective, most of the surface must be covered with PEG. ${ }^{82}$ In other words, the PEG surface concentration, and consequently grafting density, needs to be sufficiently high. Malmsten et. al. studied the effect of chain density of various PEGbased coatings on fibrinogen adsorption and found that grafted PEG demonstrates better non-fouling behaviour than those adsorbed on the surface ${ }^{83}$ (Figure 2-6). 


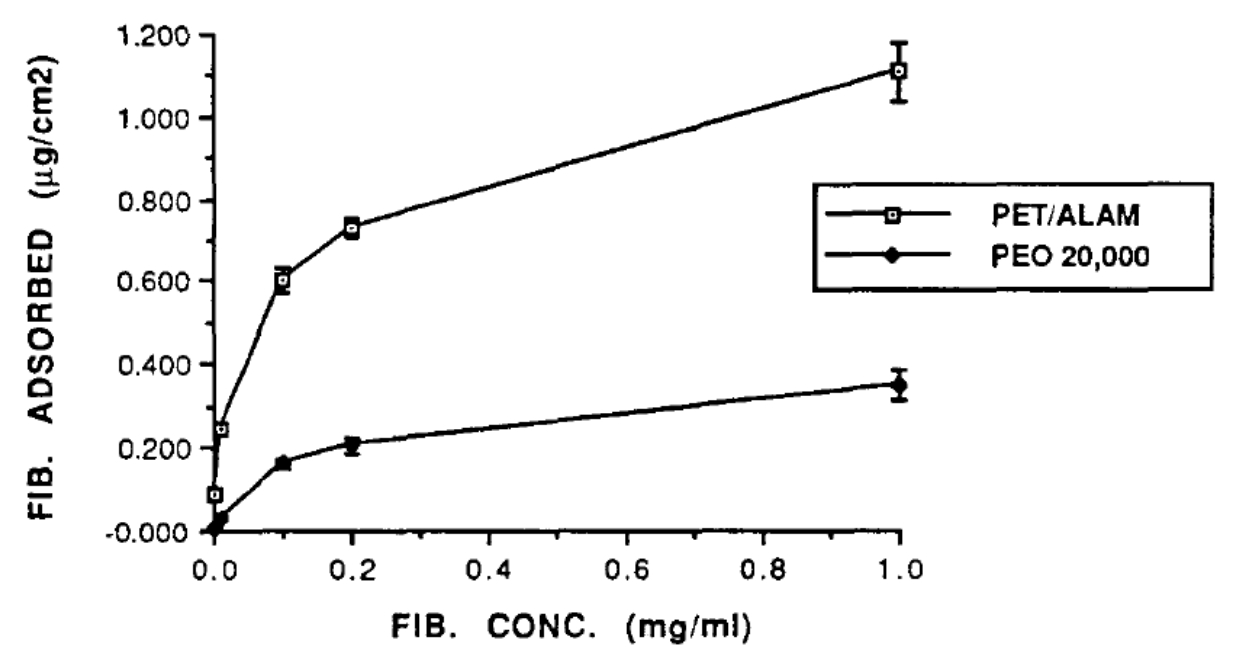

Figure 2-4. A comparison of fibrinogen adsorption isotherms before (open squares) and after (solid diamonds) the attachment of poly(ethylene oxide) (PEO) to poly(ethylene terephthalate)/allylamine (PET/ALAM) coatings. The attachment of PEO clearly reduced the amount of fibrinogen adsorbed across different concentrations. Reprinted with permission from J. Biomed. Mater. Res., 1991, 25, pp 1547-1562, reference [75]. Copyright 1991 John Wiley \& Sons.

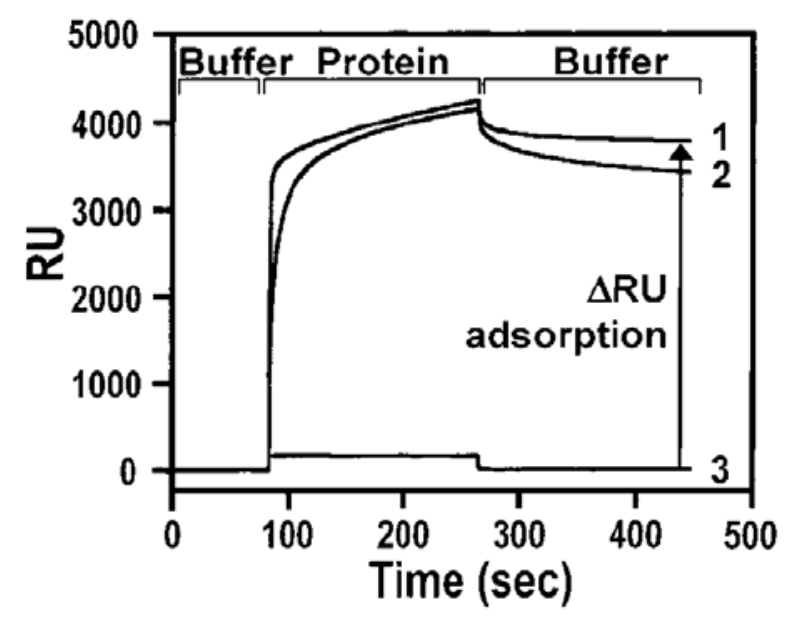

Figure 2-5. The use of SPR to characterize the non-fouling behaviour of poly(ethylene oxide) (PEO)-treated surfaces - SPR sensograms illustrating the adsorption of fibrinogen to different self-assembled monolayers (SAM). The surface of linear poly(ethylenimine)-oligo ethylene glycol LPEI-CO $\left(\mathrm{CH}_{2}\right)_{11} \mathrm{CH}_{3}$ (25kDa) adsorbs nearly a full monolayer of protein (curve 1). A mixed SAM that presents unfunctionalized LPEI also adsorbs almost a full monolayer of protein (curve 2). 
Acylation of the SAM/LPEI with acyl chloride derivative of oligo(ethylene glycol) $\mathrm{ClCOCH}_{2}\left(\mathrm{OCH}_{2} \mathrm{CH}_{2}\right)_{2} \mathrm{CH}_{3}$ generates a surface that resists the adsorption of protein (curve 3). The amount of adsorbed proteins is defined as the difference in signal between the end of the experiment and the beginning of the injection of protein. The difference is illustrated with an arrow labeled $\Delta R U$ adsorption. Reprinted with permission from Langmuir, 2001, 17 (4), pp 1225-1233, reference [78]. Copyright 2001 American Chemical Society.

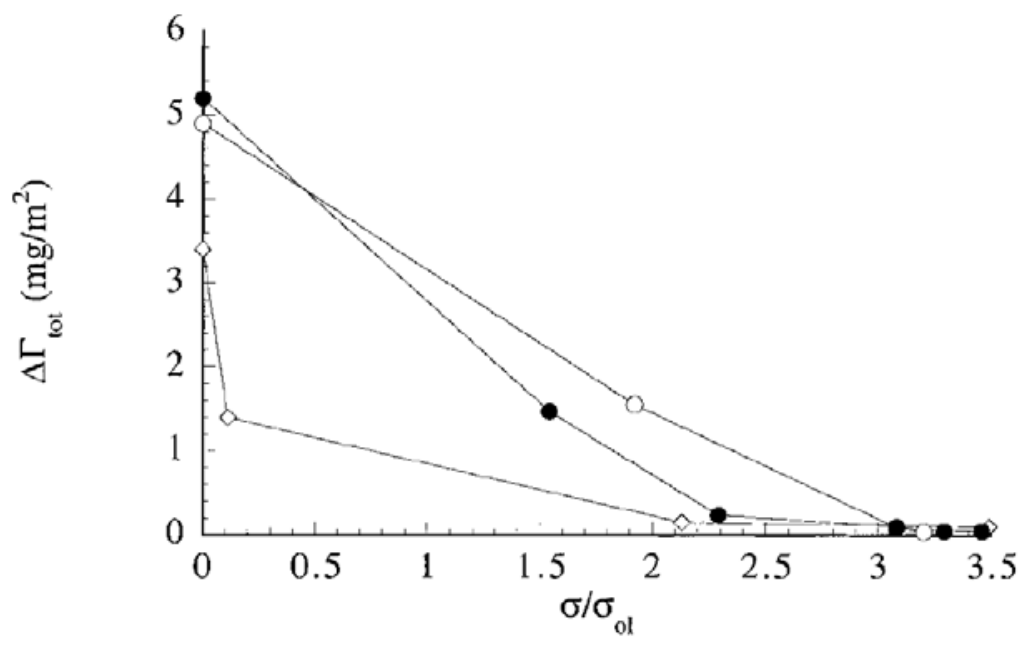

Figure 2-6. Effect of chain density on inhibition of protein adsorption by poly(ethylene glycol) (PEG) based coatings - amount of fibrinogen adsorbed (Y-axis) as a function of interfacial PEG chain density (X-axis), for monoepoxy PEG 5000 grafted at aminopropylsilane-treated silica (diamonds), PEG esterified fatty acid esters adsorbed at methylated silica and phosphatidic acid (open and filled circles respectively). These results also show that grafted PEG demonstrates better nonfouling behaviour than those adsorbed on the surface. Reprinted with permission from J. Colloid Interface Sci., 1998, 202 (2), pp 507-517, reference [83]. Copyright 1998 Elsevier.

A separate work by Halperin categorized the adsorption of proteins to polymer brush-modified substrates into two modes, namely primary adsorption and secondary adsorption. ${ }^{84}$ Primary adsorption occurs when short-range attraction is dominant. This type of adsorption is relevant for small proteins and increasing the grafting density can 
effectively reduce it. On the other hand, secondary adsorption relies on van der Waals attraction and occurs at the outer edge of the brush. This type of adsorption is relevant for bigger proteins and increasing the brush thickness can effectively reduce it.

Aside from theoretical works, experimental works have also been rigorously conducted to confirm the non-fouling properties of PEG. For example, the group of John Brash investigated the effect of surface chain density of chemisorbed PEG on the adsorption of fibrinogen and lysozyme ${ }^{85,86}$. Brash and co-workers also performed a separate experimental study using a zwitterionic polymer brush poly(2(methacryloyloxy)ethyl phosphorylcholine) (PMPC) to investigate how protein repulsion is affected by grafting density and chain length ${ }^{86,87}$. They concluded that the non-fouling behaviour is largely determined by the hydration state of the polymer; in other words the volume of water the polymer coating can retain on the surface. This is also supported by other experimental works ${ }^{83,88-91}$ and is consistent with the simulation works done by Grunze and co-workers ${ }^{92,93}$. Based on this, it is intuitive that using polymer brush for protein repulsion would be advantageous since it can be grown with tunable grafting density and chain length, allowing better control to achieve the optimal hydration state.

At present, non-fouling polymer brushes can be broken down into neutral and zwitterionic brushes. Examples of neutral non-fouling brushes includehydroxyethyl methacrylate (PHEMA) ${ }^{94}$ and poly(oligo ethylene glycol) methacrylate (POEGMA) ${ }^{95}$. They represent an intuitive choice since they can form, via SI-CRP, ultrathin polymer films similar to poly(ethyleneglycol) (PEG), which is widely known for its biocompatibility and non-biofouling properties ${ }^{96}$. In fact, for the case of POEGMA, the side chains consist of ethylene glycol repeat units. The hydroxyl group of these monomers also allows for postmodification. Another interesting prospect of 
POEGMA, as shown in this work, is the ability for the ethylene glycol repeat units to coil up and form a pseudo-ether crown structure which has an affinity towards gold. This allows the convenient incorporation of gold nanoparticles into the brush. A schematic showing the synthesis of POEGMA via SI-ATRP, along with the chemical structure is depicted in Figure 2-7.

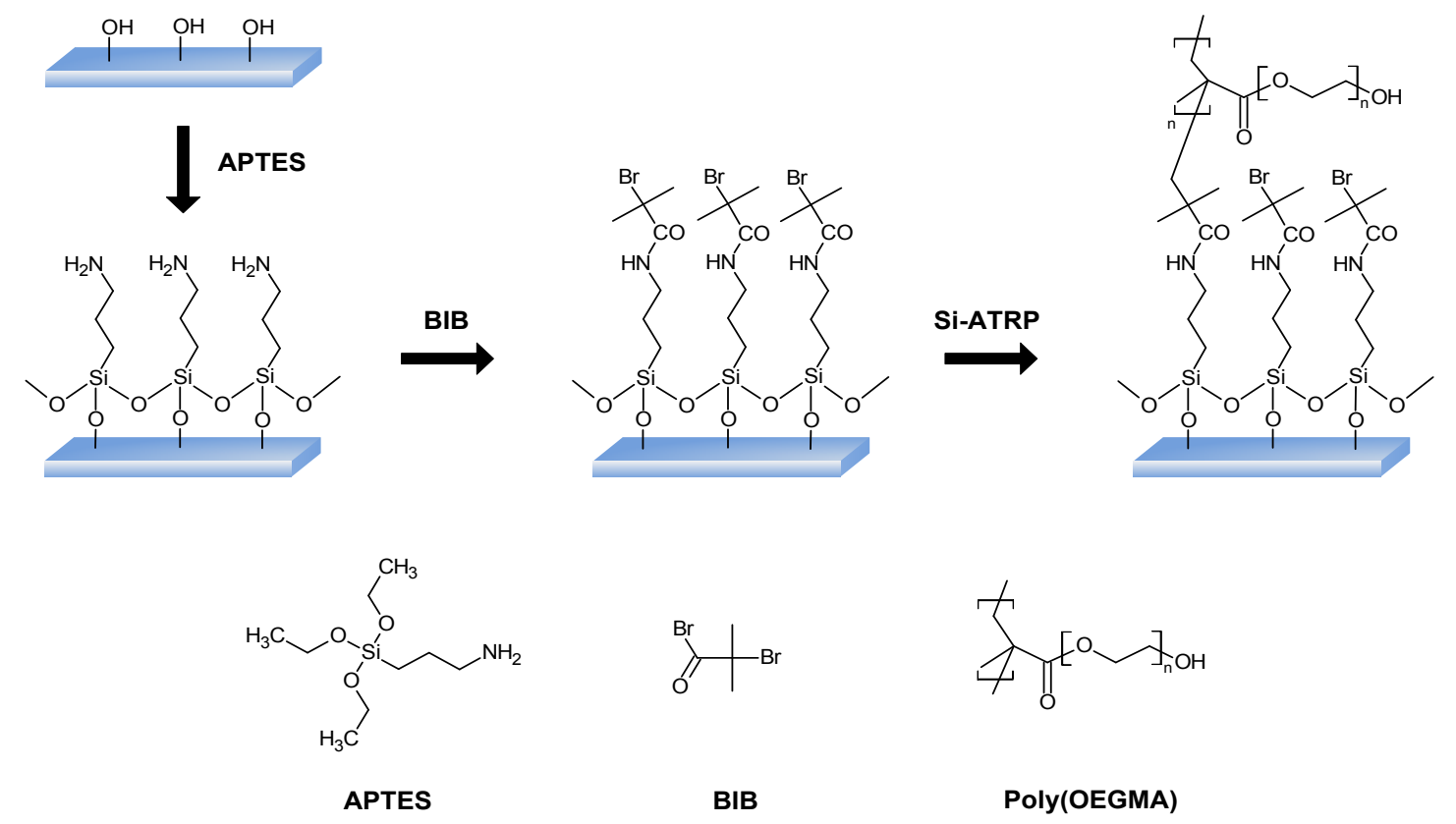

Figure 2-7. Schematic illustration of the synthesis of POEGMA brushes on glass via surface-initiated atom-transfer radical polymerization (ATRP). Cleaned slides were functionalized with aminopropyltriethoxysilane (APTES), modified to present an ATRP initiator, bromoisobutyryl bromide (BIB). Slides were then immersed in a polymerization solution to synthesize surface-tethered brushes of POEGMA.

Another monomer of interest, with respect to non-fouling polymer brushes, is NIPAM. PNIPAM is mainly attractive due to the fact that it has a lower critical solution temperature (LCST). This allows surfaces which are coated with PNIPAM to switch properties by adjusting the temperature ${ }^{97}$. This allows the user to switch the surface from hydrophilic to hydrophobic; enabling the control of release and capture 
of cells and proteins of interest ${ }^{98}$. Recently, a combination of two types of monomers such as, POEGMA and PNIPAM, has also been gaining interest to achieve a double phase transition temperature ${ }^{99}$. In addition to the neutral polymer brushes, a second major class of nonbiofouling polymer brushes that can be obtained by SI-CRP is zwitterionic polymer brushes ${ }^{100,}{ }^{101}$. Comparable to neutral polymer brushes, zwitterionic polymer brushes can also form hydrated ultrathin coatings that introduce enthalpic and entropic barriers to nonspecific adsorption of proteins and cells. Examples of zwitterionic polymer brushes include poly(2-(methacryloyloxy)ethyl phosphorylcholine) (PMPC) ${ }^{102}$, polysulfobetaine methacrylate (PSBMA) and poly(carboxybetainemethacrylate) (PCBMA) ${ }^{103,104}$.

\subsection{Noble metal nanoparticles}

Noble metal nanoparticles have been gaining increasing attention due to several interesting properties they possess ${ }^{105-109}$. In particular, they possess surface plasmon resonance properties that allow them to be good optical transducers ${ }^{110}$. Noble metal nanoparticles, or classically defined as colloidal nanoparticles, are particles which has sizes smaller than one micron or, more significantly, smaller than the wavelength of light. When light is incident on the metallic nanoparticles, it induces a collective oscillation of the conduction electrons of which the frequency depends on the shape, size and composition. This is what is known as localized surface plasmon resonance (LSPR). Due to this phenomenon, noble metal nanoparticles absorb and scatter light with tremendous intensity. The characteristic shape of the nanoparticle absorption or extinction and scattering spectra, or sometimes simply referred to as LSPR spectra, not only depends on the shape, size and composition but also on the orientation and local dielectric environment. The dependency on the local dielectric environment is particularly useful as this allows subtle changes in the environment to be detected 
through shifts in the LSPR peak wavelength. Based on this, nanoparticles can be employed, either free in solution or immobilized on a surface ${ }^{111}$, as molecular sensing modules. Briefly, the nanoparticle can be functionalized with a recognition moeity that could specifically capture the target of interest ${ }^{112}$. The attachment of target molecules on the nanoparticles would then induce a gradual shift in the LSPR spectra, which when quantified would give a correlation with the target concentration. Alternatively, a detection scheme can be developed to induce the rearrangement of nanoparticles. Such rearrangement of nanoparticles, classically through nanoparticle aggregation in solution $^{113,114}$ would induce a much greater LSPR shift as a result of "plasmon coupling' effects. Plasmon coupling is described by Jain and El-Sayed as the coupling of individual plasmon oscillations on proximal particles via their near-field interaction $^{115}$.

Nanoparticles can primarily be divided into two categories based on their shape isotropic or anisotropic. Isotropic nanoparticles simply refer to nanospheres while anisotropic nanoparticles refer to any shape having two or more dimensions. This includes nanorods, nanocubes, nanotriangles, nanopyramids and other multidimensional shaped nanoparticles. The most significant difference between isotropic and anisotropic nanoparticles lies in their LSPR spectra. Most often, anisotropic nanoparticles display more than one plasmonic peak in their spectra ${ }^{116,117}$. For instance, while gold nanospheres display a single absorption peak in the wavelength region of around 520-540 nm, gold nanorods display two peaks namely the transverse and longitudinal peaks. The existence of these two peaks arises from the oscillation of the conduction electrons along the short and long axis of the nanorod. Since nanoparticle dimension determine peak position, the longitudinal peak position of the nanorod can be adjusted by changing the 'aspect ratio' of the nanorod during 
synthesis $^{118}$. The aspect ratio is the ratio between the length and width of the nanorod; increasing the aspect ratio would shift the longitudinal peak position to higher wavelengths. Due to such tunability, along with the fact that longitudinal peak at higher wavelengths is more sensitive to changes in the dieelectric environment, nanorods are typically deemed more favorable than nanospheres when used in biological applications such as biosensing ${ }^{119}$ and photothermal therapy ${ }^{120,121}$.

Noble metal nanoparticles can be synthesized via several pathways. There are generally two methods for the synthesis of nanoparticles. They are bottom-up ${ }^{122-124}$ or top-down ${ }^{125-127}$ approaches, with some works reporting a combination of both ${ }^{128-130}$. In the bottom-up approach, the nanoparticles are formed from its constituent atoms and molecules one atom or molecule at a time. On the contrary, in the top-down approach, the synthesis begins with the removal of material from a larger nano- or microstructure. This is done via techniques such as wet etching ${ }^{131,132}$, selective metal etching $^{133,134}$, selective dealloying ${ }^{135}$, anisotropic dissolution as well as thermal decomposition $^{127}$. Generally, bottom-up approaches are more favorable due to their ability to form nanoparticles with uniform and well-defined shape and size. These approaches mainly rely on wet chemical techniques ${ }^{136-138}$ that provide much better control during the course of nanoparticle formation ${ }^{123,139}$. The combination of both top-down and bottom-up approaches is usually exploited to obtain well-defined arrays of nanostructures ${ }^{130,140-144}$. The top-down approach is used to lithographically fabricate templates that are later used for precise localization of nanostructures synthesized separately via bottom-up wet chemical techniques.

The most commonly used method for noble metal nanoparticle synthesis involves the reduction of a precursor salt by a reducing agent such as citrate ${ }^{145,146}$ or ascorbic $\operatorname{acid}^{147,148}$, either with ${ }^{149-153}$ or without a seed particle ${ }^{154-157}$. For example, in the 
classical method developed by Turkevich, the addition of sodium citrate to a solution of chloroauric acid at $100{ }^{\circ} \mathrm{C}$ resulted in the formation of gold nanoparticles with relatively uniform size of around $20 \mathrm{~nm}$ in diameter ${ }^{155}$. The founding of anisotropic nanoparticles began when El-Sayed et. al. introduced the method to synthesize gold nanorods via a seed-mediated growth approach ${ }^{158}$. This method involves two steps namely the formation of small spherical particles known as seed particles ${ }^{159}$ followed by the growth of these seeds through the reduction of gold ions in the presence of a new reductant and a templating agent usually in the form of a surfactant ${ }^{160-162}$. The surfactant form micelles that direct the growth of nanoparticle along one direction, at the same time acting as a stabilizing agent to prevent aggregation of nanoparticles in solution. El-Sayed and co-workers continued to demonstrate the applications of nanorods that have been synthesized, particularly for bioimaging and photothermal therapy ${ }^{115,118,163}$. At the same time, among the groups that had actively employed the seed-mediated growth method was that of Catherine Murphy; Murphy and co-workers demonstrated the formation gold nanorods of various aspect ratios using the seedmediated approach ${ }^{122,136,137,161,164-167}$. Since then, many works have modified this initial approach to yield a phletora of anisotropic nanostructures such as nanocubes ${ }^{168-}$ ${ }^{172}$, nanotriangles ${ }^{173-175}$, nanocages ${ }^{176}$, branched nanostructures such as nanotripods and tetrapods $^{177-180}$, as well as other complex structures ${ }^{181-187}$. Table 2-1 outlines the the most common nanoparticle structures that can be fabricated using the bottom-up as well as top-down approaches. The different shapes can be categorized as shown in Figure 2-8. 
Table 2-1. An overview of most common nanoparticle shapes that can be formed via different fabrication approaches.

\begin{tabular}{|c|c|c|}
\hline \multicolumn{3}{|l|}{ Bottom-up Approach } \\
\hline Structure & Fabrication Method & Representative ref(s). \\
\hline Nanospheres & Wet-chemical, direct reduction of metal salt & $154,155,157$ \\
\hline Nanospheres & \multirow[t]{5}{*}{ Wet-chemical, seed-mediated } & $149-153$ \\
\hline Nanorods & & $158,162,164$ \\
\hline Nanocubes & & $168-172$ \\
\hline Nanotriangles & & $173-175$ \\
\hline Nanotripods, Nanotetrapods & & $177-180$ \\
\hline \multicolumn{3}{|l|}{ Top-down Approach } \\
\hline Structure & Fabrication Method & Representative ref(s). \\
\hline Nanospheres & UV photolithography & 125 \\
\hline Nanospheres & Controlled wet etching & 131,132 \\
\hline Nanoframes & Selective metal etching & 133,134 \\
\hline Nanoframes & Dealloying & 135 \\
\hline
\end{tabular}

\section{Noble Metal Nanoparticle Shapes}

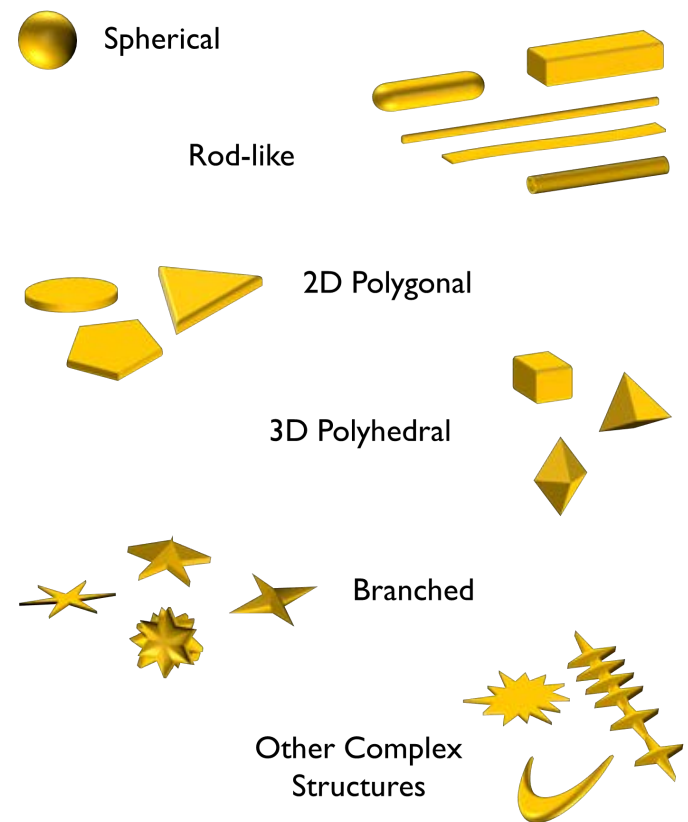

Figure 2-8. A schematic showing the different shape categories of the most common noble metal nanoparticles. 


\subsection{Polymer brush nanoparticle nanocomposites}

The mixing of polymers and nanoparticles is opening pathways for engineering flexible composites that exhibit advantageous electrical, optical, or mechanical properties. Recent advances reveal routes to exploit both enthalpic and entropic interactions so as to direct the spatial distribution of nanoparticles and thereby control the macroscopic performance of the material. For example, by tailoring the particle coating and size, researchers have created self-healing materials for improved sustainability and self-corralling rods for photovoltaic applications. A challenge for future studies is to create hierarchically structured composites in which each sublayer contributes a distinct function to yield a mechanically integrated, multifunctional material.

\subsubsection{Polymer brush nanoparticle nanocomposite construction strategies}

The construction of polymer brush nanoparticle nanocomposite is challenging particularly due to the fact that the polymer brush exerts a volummetric exclusion effect, which prevents the nanoparticles from infiltrating the brush. Several strategies have been employed. Some groups have attempted to incorporate nanoparticles in polymer brushes by modifying the surface of the nanoparticles to include the polymer $^{188,189}$. The grafting density and brush thickness can also be adjusted.

While the above methods require the nanoparticles to be separately synthesized ex-site, another approach attains nanoparticles within the brushes through the reduction of metallic salts. This was demonstrated by Choi et. al. when they showed that polyelectrolyte brushes loaded with magnetic nanoparticles could be obtained through the reaction of immobilized $\mathrm{Fe}^{2+} / \mathrm{Fe}^{3+}$ ions in the brush ${ }^{190}$. Briefly, after the anionic poly(3-sulfopropyl methacrylate) brushes were grown from gold-coated 
silicon wafers, iron precursor salts $\mathrm{FeSO}_{4}$ and $\mathrm{Fe}_{2}\left(\mathrm{SO}_{4}\right)_{3}$ were introduced at high concentrations for a period of $12 \mathrm{~h}$. The adsorbed ions were then hydrolyzed by oxygen in solution and air during dipping to result in iron oxide nanoparticles. In another work by Gupta et. al., the same approach was used to obtain silver nanoparticles in poly(2-(dimethylamino)ethyl methacrylate) PDMAEMA brushes ${ }^{191}$. After modifying the glassy carbon substrates by direct growth of PDMAEMA brushes, they were immersed and stirred in a solution of $\mathrm{AgNO}_{3}$ for $1 \mathrm{~h}$ to induce the capture of $\mathrm{Ag}^{+}$via electrostatic adsorption along the PDMAEMA chains. They were quickly transferred to a solution of $\mathrm{NaBH}_{4}$ solution to reduce the $\mathrm{Ag}^{+}$to $\mathrm{AgNPs}$. They reported homogeneous distribution of 'small' AgNPs within the polymer brush although the size distribution and morphology of resulting AgNPs was not discussed in detail.

While obtaining nanoparticles in polymer brushes via in situ reduction might yield good nanoparticle distribution, there are several clear disadvantages. Firstly, obtaining nanoparticles with excellent morphology within the polymer brush via this method would not be straightforward since there is an additional interaction with the brush chains. In other words, this might hamper the formation of polymer brush nanoparticle nanocomposite with nanoparticles of clearly defined shapes such as nanorods, nanocubes, nanotriangles and other anisotropic nanoparticles. Additionally on a related note, there is also an issue of impurity formation within the polymer brush, which can arise from incomplete reduction of the precursor salts. Secondly, this also means that the presence of additional interactions from the brush needs to be carefully taken into consideration. So far, in the works discussed earlier, it is possible to obtain the nanoparticles via reduction since the precursor salts have strong affinity towards the polymer brush units. However, this is not always the case. For example, if the application demands that a cationic polymer brush is to be used, the positive charges 
from the polymer brush would hamper the adsorption of most metallic precursor salts. The brush interactions could also be counter-productive during the precursor reduction step since they can impede the access of reducing agent and prevent fully uniform and isotropic nanoparticle growth. Thirdly, it is also difficult to control the nanoparticle vertical distribution within the polymer brush since it is difficult to determine the extent of precursor salt infiltration in the first place, unless a block copolymer system is utilized to specifically localize, at a certain depth, a polymer brush with an affinity towards the precursor. Finally, the growth of nanoparticles from within the polymer brush could also deplete the pristine properties of the brush. As there is a chemical step involved during the reduction step, the chains of the brush have the possibility of being involved in a cross-reaction or even result in being degraded.

On the flipside, the mentioned disadvantages are generally irrelevant for the preparation of polymer brush nanoparticle nanocomposite through ex situ nanoparticle synthesis, since preparation via this method typically does not involve any chemical reaction within the brush. Nanoparticles can be carefully tailored and synthesized with excellent size distribution and shape uniformity, and with complete removal of any impurities prior to immobilization on the brush. Since there is a huge collection of established nanoparticle synthesis protocols in literature, the attainment of anisotropic nanoparticles would be fairly straightforward. However, there still exist several difficulties associated with this method. The difficulties primarily stem from the volummetric exclusion effect of the brush. Since the dimensions of nanoparticles are much larger than the inter-grafting distance of the polymer brush chains, the polymer brush would have the tendency to exclude the nanoparticles from the interface, preventing infiltration. In order to circumvent this, the polymer brush surface would need to be modified, in effect introducing an additional step to the methodology. And 
while such post-modifications are common and simple for the case of isotropic nanoparticles such as gold, where the as-prepared nanoparticles are stabilized in citrate, the modification of anisotropic nanoparticles is much more tedious since most anisotropic nanoparticles are prepared in surfactants which adhere strongly to the surface. In addition, anisotropic nanoparticles, due to their two-dimensional nature, resist infiltration much more than isotropic nanoparticles.

\subsubsection{Polymer brush nanoparticle nanocomposite applications}

Nanoparticles in end-tethered polymer brushes have been employed in various applications. For example, Snaith et. al has reported the assembly of CdSe nanocrystals in polyacrylate brushes grown from indium tin oxide ${ }^{192}$. The assemblies attained show good nanoparticle distribution in the mesoscale order and they have demonstrated quantum efficiencies of up to $50 \%$. Choi and co-workers also found that small ferromagnetic nanoparticles behave in a unique way when embedded in polyelectrolyte brushes. In particular, they observed that such embedment affects the way the brushes behave and found that the brushes were able to change dimensions in a reversible manner when subjected to an external magnetic force. Reversible properties are also useful when detecting environmental changes be it in solution or even air. For example, nanoparticles in polymer brushes can be utilized to sense changes in the local environment such as temperature and $\mathrm{pH}$ as well as the presence of certain molecular targets. One of the earlier works that explored the feasibility of pH sensing was done by Tokareva et. al. a decade ago in which a combination of gold nano-islands, gold nanoparticles and poly(2-vinylpyridine), P2VP brushes was expoited $^{193}$. Briefly, the P2VP brushes were grown from the gold nano-islands and the gold nanoparticles were fixed to the free end of the brush. As the P2VP brushes would swell when $\mathrm{pH}$ is lowered down to $\mathrm{pH} 2$ and collapse when $\mathrm{pH}$ is increased up to $\mathrm{pH}$ 
5, the distance between the nano-islands and the nanoparticles would vary accordingly. Since plasmon coupling arises when the nanoparticles are in close proximity to the nano-islands, transmission surface plasmon resonance (T-SPR) was used to detect $\mathrm{pH}$ changes. They demonstrated a $50 \mathrm{~nm}$ shift of the absorption maximum when $\mathrm{pH}$ is varied between the two extremities, in both directions. While still exploiting the reversible swelling property of polymer brushes, a slightly more straightforward approach was demonstrated by Gupta et. al. to detect temperature changes. In this case, they omitted the use of nanoislands on the substrate and simply immobilized functionalized gold nanoparticles in poly( $\mathrm{N}$-isopropyl acrylamide) PNIPAAm. Since PNIPAAm has a lower critical solution temperature (LCST) of $40^{\circ} \mathrm{C}$, the brushes would collapse when heated above the LCST, forcing the immobilized gold nanoparticles to be squeezed much closer to each other. This decrease in the interparticle distance led to a red shift in the SPR signal. Consequently, when temperature is lowered below the LCST, the brushes would swell leading to an increase in interparticle distance and a blue shift in the SPR signal. As with the work done by Tokareva et. al., the shifts with respect to temperature modulation is found to be reversible, with a maximum shift of around $12 \mathrm{~nm}$ as detected via UV-vis spectroscopy. In terms of peak shift, they also mentioned a comparison with an earlier work by Misuishi et. al., which utilized the same PNIPAAm-Au system but in a slightly different configuration. In that work, the gold nanoparticles were embedded on the surface and the polymer brush is grown from surfaces that are not blocked by the nanoparticles. Hence, the collapse and swelling does not affect the cofiguration of nanoparticles with respect to each other with absolutely no change in interparticle distance. The SPR shifts arise from the change in refractive indices when the brush engulfs the nanoparticles in collapse state. As a result this gave a lower peak shift of 
around $6 \mathrm{~nm}$. In addition, Tokareva et. al. also mentioned in their work that the difference in the magnitude of the peak shift was also attributed to the size difference of immobilized gold nanoparticles; the small the nanoparticles the higher the mobility and resulting peak shift.

Besides detecting the changes in environment such as $\mathrm{pH}$ and temperature of the solution, nanoparticles embedded in polymer brushes have also been shown to be useful for molecular sensing. One of the methods to achieve this relies on surface enhanced raman spectroscopy (SERS). Since the Raman signature spectra of molecules can be tremendously enhanced when they are positioned at junctions of silver and gold nanoparticles, obtaining a large number of nanoparticles assembled close to each other would lead to a higher probability of molecules being adsorbed

within these areas. In the work by Gupta et. al., silver nanoparticles immobilized in poly(2-(dimethylamino)ethyl methacrylate) brushes directly grown from glassy carbon was used to detect the commonly used SERS probe, Rhodamine 6G. They reported a surface enhancement factor of $6 \times 10^{6}$.

\subsection{Future Perspectives}

Over the past decade, several methodologies have been established to enable the controlled synthesis of polymer brushes and nanoparticles, as well as the incorporation of nanoparticles within polymer brushes. As a result, many works have demonstrated the potential of substrate-bound polymer brush nanoparticle nanocomposites in a variety of applications - by exploiting the electrically conductive, magnetic and, most commonly, the optical properties of the embedded metal nanoparticles as well as the stimuli responsiveness of the polymer brush along with its biocompatibility and nonfouling properties. 
In most of these cases however, the respective properties of the two classes of materials are somewhat compartmentalized, with emphasis being put on one class of material. From a host-guest perspective, it is more often the properties of the guest (i.e. nanoparticles) that are of primary interest, with the host (i.e. polymer brush) acting mainly as a scaffold. For example, in molecular sensing, the final signal readout stems from either the LSPR shifts from the rearrangements of nanoparticles or the SERS electromagnetic enhancements arising from the junctions between nanoparticles. In general, there is not much value-addedness of both classes of materials upon each other, other than to support the nanoparticles on a surface with high loading.

What needs to be shown more is how the polymer brush can introduce new kinds of behaviours to the nanoparticles which otherwise cannot be observed in a free system (either in solution or immobilized on a substrate). One aspect is controlled aggregation in the form dimer, trimer and multimer arrays on a surface. The introduction of functional groups to the polymer brushes can be explored to induce localized mobility of nanoparticles on surfaces with polymer brushes. While a lot of work has been conducted based on the motion of nanoparticles fixed to the polymer brush chains, and primarily exploiting the configurational changes of the polymer brush, the total re-arrangement of nanoparticles within the polymer brush has yet to be fully explored. Such total re-arrangements need to rely on functional groups on the brush that can strike a delicate balance between nanoparticle adhesion and release. Based on this, two approaches can be projected. Firstly, a stimulus in the form of a molecular target of interest can be introduced to bind to the nanoparticle more strongly than the brush, leading to the release of nanoparticle from the brush followed by controlled aggregation occuring in the bulk solution before finally leading to the reattachment of the aggregate on the polymer brush. Alternatively, the molecular target 
can bind to the polymer brush itself, at a certain designated region, consequently displacing the nanoparticles to other regions where the brush still have affinity for the nanoparticles. For each of these cases, a homogenous and a block copolymer system may be used, respectively.

A more radical step forward would be to explore sustainable reversible shapeshifting of nanoparticles within polymer brushes. For example, metallic anisotropic nanoparticles such as gold nanorods could be leached to form gold nanospheres in the presence of a particular target of interest. Instead of losing the gold ions to the bulk solution, the polymer brush can be used to trap the ions the same way precursor ions adsorbed on polymer backbones as reported by earlier works. At the same time, a reducing agent can also be integrated within the polymer brush system, such that when detection is completed and the target is released, the localized gold ions can be reduced again from the nanospheres acting as cores to reform the nanorods. As the formation of nanorods is usually a complex process involving surfactants as shapedirecting agents, it would be beneficial in this case to explore the ability of the brush to replace the role of surfactants in situ.

These schemes involving controlled aggregation of nanoparticles as well as shapeshifting nanoparticles would largely be beneficial in the development of molecular sensing platforms. Not only would this lead to an increase in detection sensitivity arising from much larger, more distinct shifts in LSPR signals; it would also pave the way for construction of economical sensing devices which could eventually be used in point-of-care settings. Huge shifts in LSPR signals translate to a clear observable color change especially when the platform is grown on a transparent substrate such as glass, enabling naked eye detection. Taking into account the use of excellent non-fouling polymer brushes, such economical and easy-to-use sensing platforms can receive 
complex bodily fluids such as blood, urine and saliva for direct and rapid detection of any disease of interest. 


\section{Chapter 3}

\section{Materials and Methods}

\subsection{Common Materials}

\subsubsection{Chemicals and solvents}

2-mercaptoethanol (2-ME), 2,2'-bipyridyl (bpy), aminopropyltriethoxysilane (APTES, >99\%), ascorbic acid (AA), bromoisobutyryl bromide (BiB), cetyltrimethylammonium bromide $(\mathrm{CTAB},>99.9 \%)$, ethanol $(>99 \%)$, hydrochloric acid (HCl), lead(II) nitrate, poly(ethylene glycol) methacrylate, polystyrenesulfonate (PSS), rhodamine 6G (R6G), sodium borohydride $\left(\mathrm{NaBH}_{4}\right)$, sodium chloride $(\mathrm{NaCl})$, sodium thiosulfate, silver nitrate $\left(\mathrm{AgNO}_{3}\right)$, tetrachloroauric acid $\left(\mathrm{HAuCl}_{4}\right)$, triethylamine, trisodium citrate, and all metallic salts used were purchased from Sigma-Aldrich. Tris[2-carboxyethyl] phosphine (TCEP) were purchased from Merck Pte. Ltd. Dichloromethane (HPLC grade) and methanol (>99\%) were purchased from Fisher Scientific. All chemicals were used without further purification.

\subsubsection{Biomolecules and buffer solutions}

Sandwich immunoassay was performed using mouse monoclonal anti-human IgG as the capture antibody, purified human IgG as target and AlexaFluor 546-goat antihuman $\operatorname{IgG}$ as detection antibody; all purchased from Invitrogen, Life Technologies. Human serum from human male $\mathrm{AB}$ plasma, sterile-filtered was purchased from Sigma. Thrombin binding aptamer (TBA, 5'-C6-S-S-(T)5-GGT TGG TGT GGT TGG-3') was synthesized by Sigma-Aldrich and human $\alpha$-thrombin was purchased from Abcam. 
Glycine-NaOH buffer was prepared from $50 \mathrm{mM}$ Glycine solution with $\mathrm{pH}$ adjusted to 10 using $1.0 \mathrm{~N} \mathrm{NaOH}$. Washing buffer consisted of TBS with $0.1 \% \mathrm{v} / \mathrm{v}$ Tween 20 and thrombin binding buffer comprises $50 \mathrm{mM}$ Tris- $\mathrm{HCl}(\mathrm{pH} 7.4)$, $140 \mathrm{mM} \mathrm{NaCl}, 1 \mathrm{mM} \mathrm{MgCl} 2$ \& $100 \mathrm{mM} \mathrm{KCl}$. Milli-Q-ultrapure was used in all buffer preparation steps.

\subsubsection{Substrates}

Glass coverslips $\left(18 \times 18 \mathrm{~mm}^{2}\right.$ and $\left.22 \times 22 \mathrm{~mm}^{2}\right)$ and glass slides $(76 \mathrm{~mm} \times 26$ $\mathrm{mm}$ ) were purchased from Fisher Scientific. All glass coverslips, glass slides and oxidized silicon wafer substrates were cleaned in piranha solution of $\mathrm{H}_{2} \mathrm{SO}_{4} / \mathrm{H}_{2} \mathrm{O}_{2}$ (3:1) for 20 min prior to any further surface modifications.

\subsection{General Methods}

\subsubsection{Polymer brush synthesis}

The polymer brush poly(oligo theylene glycol methacrylate) was synthesized on silicon chips and glass coverslips according to previous report. Glass coverslips $(18 \mathrm{x}$ $18 \mathrm{~mm}^{2}$ ) and oxidised silicon wafer substrates were first cleaned in Piranha solution of $\mathrm{H}_{2} \mathrm{SO}_{4} / \mathrm{H}_{2} \mathrm{O}_{2}$ (3:1) for 20 min. After they were copiously rinsed with deionized $\mathrm{H}_{2} \mathrm{O}$ and blown dry with $\mathrm{N}_{2}$, they were immersed in aminopropyltriethoxysilane $(10 \% \mathrm{v} / \mathrm{v})$ in ethanol for $1 \mathrm{~h}$. They were then rinsed with copious amount of ethanol, blown dry with $\mathrm{N}_{2}$ and baked in an oven at $120{ }^{\circ} \mathrm{C}$ for $1 \mathrm{~h}$. The substrates were then immersed in a solution of bromoisobutyryl bromide $(370 \mu \mathrm{L})$, and triethylamine $(410 \mu \mathrm{L})$ in dichloromethane $(60 \mathrm{~mL})$ for $30 \mathrm{~min}$, rinsed with dichloromethane and ethanol and blown dry with $\mathrm{N}_{2}$. Polymerization was performed by immersing the substrates at room temperature in a degassed solution of $\mathrm{Cu}(\mathrm{I}) \mathrm{Br}(143 \mathrm{mg}), 2$,' '-bipyridyl (312 mg) and OEGMA $(8.6 \mathrm{~mL})$ in methanol $(12 \mathrm{~mL})$ and deionized water $(3 \mathrm{~mL})$ under $\mathrm{N}_{2}$ 
purge for $24 \mathrm{~h}$. Finally the substrates were removed, thoroughly rinsed with methanol and blown dry with $\mathrm{N}_{2}$.

\subsubsection{Gold nanoparticle synthesis}

AuNPs ( 4 $\mathrm{nm}$ in diameter) were synthesized via borohydride reduction of tetrachloroauric acid. Briefly, $0.6 \mathrm{~mL}$ of ice-cold, freshly prepared $0.1 \mathrm{M} \mathrm{NaBH} 4$ solution was added dropwise to a $20 \mathrm{~mL}$ solution containing $2.5 \times 10^{-4} \mathrm{M} \mathrm{HAuCl}_{4}$ and $2.5 \times 10^{-4} \mathrm{M}$ of trisodium citrate under vigorous stirring in a round-bottom flask at room temperature. The formation of AuNPs after the addition of $\mathrm{NaBH}_{4}$ eventually gave rise to a pinkish red solution.

AuNPs ( $\sim 15 \mathrm{~nm}$ in diameter) were synthesized via Turkevich method. Briefly, 40 $\mu \mathrm{L}$ of $2.5 \mathrm{M} \mathrm{HAuCl}_{4}$ and $100 \mathrm{~mL}$ of Millipore water were added to a two-neck flask. A condenser was attached and the setup was allowed to reflux. Then, $10 \mathrm{~mL}$ of 38.8 $\mathrm{mM}$ of sodium citrate was quickly added before allowing further reflux for an additional $20 \mathrm{~min}$. The setup was eventually allowed to cool to room temperature before AuNPs were retrieved.

\subsubsection{Gold nanorod synthesis}

The AuNRs were synthesized using the seed-mediated growth method. Briefly, 10 $\mathrm{mL}$ of $0.2 \mathrm{M}$ CTAB solution was first mixed with $10 \mathrm{~mL}$ of $5 \times 10^{-4} \mathrm{M} \mathrm{HAuCl}_{4}$. Then, $1.2 \mathrm{~mL}$ of ice-cold $0.01 \mathrm{M} \mathrm{NaBH}_{4}$ was added to the mixed solution with vigorous stirring for 2 min. The obtained solution exhibited a brownish-yellow color and was kept at room temperature for at least $10 \mathrm{~min}$ before further use. Next, the growth solution was prepared by first mixing aqueous solutions of $\mathrm{HAuCl}_{4}(0.01 \mathrm{M}, 4 \mathrm{~mL})$, $\mathrm{AgNO}_{3}(0.01 \mathrm{M}, 0.8 \mathrm{~mL})$ and $\mathrm{CTAB}(0.1 \mathrm{M}, 80 \mathrm{~mL})$. A freshly prepared aqueous ascorbic acid (AA) solution $(0.1 \mathrm{M}, 0.64 \mathrm{~mL})$ was then added followed by the addition 
of aqueous $\mathrm{HCl}$ solution $(1.0 \mathrm{M}, 1.6 \mathrm{~mL})$. After the resultant solution was mixed by inversion, the seed solution $(0.02 \mathrm{~mL})$ was added. The reaction mixture was subjected to gentle inversion for $10 \mathrm{~s}$ and then left undisturbed for at least $6 \mathrm{~h}$. The ionic strength of the as-grown AuNR solution was then adjusted by adding $\mathrm{NaCl}$ just before further use. Briefly, the AuNR solution was centrifuged at $8000 \mathrm{rpm}$ for $10 \mathrm{~min}$ and redispersed in water to remove excess CTAB. This process was repeated two more times, where in the final round, the redispersion was done using $\mathrm{NaCl}$-spiked $\mathrm{DI}_{2} \mathrm{O}$ to give the AuNR solution a final predetermined concentration of $\mathrm{NaCl}$. To achieve moderate and high $\mathrm{CTAB}$ concentrations, redispersion after the second round of centrifugation was done in deionized (DI) water using a 1:10 and 1:5 supernate surfactant/water dilution factor, respectively. To achieve low CTAB concentration, almost all the supernate surfactant is removed by carefully pipetting it out. To obtain higher concentrations of AuNR, the final redispersion was done using a lower volume of NaCl-spiked DI $\mathrm{H}_{2} \mathrm{O}$. 


\section{Chapter 4}

\section{POEGMA as a Non-Fouling Support Matrix for Ultrasensitive Protein Sensing}

\subsection{Introduction}

Substrate-based assays currently play an increasingly vital role in disease diagnostics and proteomic studies particularly due to their promising high-throughput and multiplexing capabilities ${ }^{194-200}$. Nevertheless, in the development of antibody microarrays for clinical use, non-specific adsorption of proteins can severely limit the sensitivity especially when testing from samples of blood or plasma ${ }^{201,202}$. Various methodologies aimed at ensuring high sensitivity and specificity have been established principally via the reduction of non-specific protein adsorption on substrate surfaces $^{203-206}$. In parallel, numerous strategies have been unveiled in an attempt to achieve high density immobilization of protein molecules ${ }^{207}$. These strategies, among others, employ covalent binding ${ }^{208-210}$, electrostatic adsorption ${ }^{211,212}$ as well as binding to metal complexes ${ }^{213-215}$. Although popular chemical immobilization methods utilizing covalent attachment schemes, such as amineand carbodiimide (CDI) chemistry, are traditionally deemed more reliable than physical methods ${ }^{210}$, an additional step is often required to convert the end groups into functional groups capable of anchoring the proteins.

The development of a well-defined poly(oligo(ethylene glycol) methacrylate) (POEGMA) has been gaining considerable attention over recent years ${ }^{210,213,216-226}$. These polymer brushes, which can be conveniently synthesized in a controllable manner via surface-initiated atom transfer radical polymerization (SI-ATRP), have 
been proven remarkably capable of eliminating non-specific adsorption ${ }^{201}$. Besides its non-fouling properties, POEGMA permits physical entrapment of capture probes on the surface. Recently, Hucknall and co-workers have demonstrated the operability of microarrays utilizing capture antibodies which are physically immobilized onto glass substrates coated with POEGMA ${ }^{227}$. The extreme simplicity of such noncovalentimmobilization strategy, which requires no slide activation and deactivation steps, presents a clear advantage over chemical immobilization methods. In addition, they also found that the shelf-life of POEGMA-coated glass slides had minimal impact on the spot quality and subsequent performance of the arrays, as opposed to chemically activated slides which tend to deteriorate over time. This approach is anologous to previous works on SPR biodetection in hydrogels, whereby recognition elements such as antibodies are homogeneously distributed within the hydrogel volume ${ }^{66-69}$.

Despite the apparent advantages of physical immobilization of proteins, a methodology to achieve high protein loading on protein-repulsive polymer brushes has not been well discussed. Based on a widely accepted theory that, in a wet state, a water barrier forms in polymers with ethylene glycol groups ${ }^{79,225}$, it has been hypothesized that vacuum dessication after antibody spotting results in removal of this macroscopic bulk water from POEGMA leading to protein immobilization through physical entanglement and secondary bonding interactions ${ }^{227}$. There have also been several earlier reports claiming that PEG can adapt different configurations and exist in a protein-attractive state, especially under compression ${ }^{228,229}$. Brash and co-workers also reported non-monotonous adsorption of proteins on PEO brushes which is dependent on the nature of end-group at high surface coverage $\mathrm{e}^{230}$. While the above points can explain the driving force behind physical antibody immobilization, it remains unclear 
exactly how the antibodies eventually organize within the brush, which can eventually offer a lead for the attainment of efficient protein loading.

In addition, we have yet to find any report which studied the trend of physicallyimmobilized protein loading with different brush thickness. Li and co-workers performed an investigation onprotein loading on polymer brush with varying density of amino-reactive group, glycidyl methacrylate ${ }^{231}$. While the immobilization methodology is largely physical in nature (i.e. via spot drying of protein solution on the brush under vacuum similar to the methodology used in this work), the eventual retention of the protein is due to a covalent interaction between glycidyl methacrylatemoeities and protein amino groups. In many studies which involve covalently-immobilized proteins, an increase in protein loading capacity is usually briefly accounted for by the fact that higher brush thickness allows more proteins to infiltrate into the brush ${ }^{213}, 214,224,232,233$. As far as we know, there has been no systematic attempt to support this claim by correlating with existing theoretical models that take into account geometry using actual dimensions and free energy in determining the ease with which the antibody can penetrate the brush.

As we foresee vast prospect in the physical immobilization methodology, herein we first reveal how physically immobilized antibodies organize within the polymer brush across a wide range of brush thicknesses (up to $\sim 100 \mathrm{~nm}$ ) by quantitative, qualitative, and direct visual evidences. We then correlate the experimentally observed trend to an existing theoretical model to determine whether the antibodies are adsorbed on top of the brush, infiltrated into the brush or embedded at the sub-surface of the brush, by paying attention to several critical size scales. Such insightful assessment is important to 1) determine the general practicality of physical immobilization of proteins in POEGMA and possibly other protein-repulsive polymer brushes, 2) realize 
a systematic approach which could be adopted to maximize protein loading, and 3) eventually promote physical immobilization now that it is possible to achieve a more precise control.

\subsection{Specific Methods}

\subsubsection{Antibody-spotting for fluorescence measurements}

AlexaFluor 546-goat anti-human IgG purchased from Invitrogen, Life Technologies were diluted to $200,100,50,25$ and $10 \mu \mathrm{gL}^{-1}$ in $1 \times$ PBS and $2.5 \%$ w/v glycerol before they were spotted using a Bio-Rad VersArray ChipWriter Compact System onto POEGMA-coated glass slides at room temperature and humidity. They were subsequently allowed to non-covalently absorb into polymer brush under vacuum dessication at $30 \mathrm{kPa}$ for $24 \mathrm{~h}$ at room temperature. They were then rinsed with $1 \times$ TBS with $0.1 \% \mathrm{w} / \mathrm{v}$ Tween-20 washing buffer for three times under orbital shaking at $80 \mathrm{rpm}$ for $5 \mathrm{~min}$ each before they were briefly rinsed with DI water and dried under a mild flow of nitrogen. For stability study, after the slides were dried, they were subjected to prolonged washing by immersing in $1 \times$ TBS with $0.1 \%$ w/v Tween-20 washing buffer for $1 \mathrm{~h}$ under orbital shaking at $120 \mathrm{rpm}$. Spotted arrays were then imaged and quantified after the respective treatments.

\subsubsection{Characterization}

The thickness of the polymer brush on the slides was inferred by measurements of the thickness of a POEGMA brush grown on an oxidized silicon wafer under identical conditions by ellipsometry in air using an J. A. Woolam $\alpha$-SE spectroscopic ellipsometer. Fluorescence imaging and measurements were performed using Perkin Elmer ScanArray Gx Microarray scanner equipped with ScanArray Express with Protein Array Analysis software. For atomic force microscopy (AFM) study, $1 \mu \mathrm{L}$ of 
$200 \mu \mathrm{g} \mathrm{mL}^{-1}$ antibody solution was dropped onto POEGMA brush grown on an oxidized silicon wafer before being dried under vacuum dessication and rinsed (as described above). The AFM retraces were obtained in air from an Asylum Research MFP-3D AFM system conducted in tapping mode using a SuperSharpSilicon AFM tip purchased from NanoWorld.

\subsubsection{Sandwich immunoassay}

Sandwich immunoassay was performed using mouse monoclonal anti-human IgG as the capture antibody, purified human IgG as target and AlexaFluor 546-goat antihuman IgG as detection antibody; all purchased from Invitrogen, Life Technologies. Arrays of 4 spots by 5 rows consisting of 1 row of blank PBS buffer, 1 row of $200 \mu \mathrm{g}$ $\mathrm{mL}^{-1}$ bovine serum albumin in PBS bufferand 3 rows of $200 \mu \mathrm{g} \mathrm{mL} \mathrm{m}^{-1}$ captureantibody in PBS buffer were printed onto slides grown with POEGMA for $6 \mathrm{~h}$ and vacuum dried. They were then rinsed in $1 \times$ TBS with $0.1 \% \mathrm{w} / \mathrm{v}$ Tween-20 washing buffer for three times under orbital shaking at $80 \mathrm{rpm}$ for $5 \mathrm{~min}$. A dilution series of

purified human IgG with concentrations ranging from $1 \mathrm{fg} \mathrm{mL}^{-1}$ to $10 \mathrm{ng} \mathrm{mL}{ }^{-1}$ in PBSwas then dropped onto the spotted arrays and incubated at $37{ }^{\circ} \mathrm{C}$ for $2 \mathrm{~h}$. After incubation, the slides were rinsed three times in washing buffer before the introduction of AlexaFluor 546-goat anti-human IgG and incubated at $37^{\circ} \mathrm{C}$ for $1 \mathrm{~h}$. Finally, the slides were rinsed three times with washing buffer and briefly rinsed with DI H2O before drying under mild nitrogen flow. Arrays were then imaged and quantified. Data obtained were fit to a user-defined five-parameter logistic fit performed using Origin 8.0 to obtain the dose-response curve. 


\subsection{Results and Discussion}

\subsubsection{Antibody loading as a function of brush thickness}

Arrays of spots with different concentrations of fluorescence-tagged IgG ranging from 10 to $200 \mu \mathrm{g} \mathrm{mL}^{-1}$ were machine-printed onto the substrates coated with brushes grown from $10 \mathrm{~min}$ to $24 \mathrm{~h}$, placed under vacuum dessication, rinsed and dried. The thickness of brush polymer ranged from 6 to $102 \mathrm{~nm}$ (Figure 4-1). In the plot of fluorescence intensity against thickness, as shown in Figure 4-2, a spline-shaped curve was obtained for each concentration with the fluorescence intensity starting at a maximum with minimum brush thickness and decreased until $19 \mathrm{~nm}(1 \mathrm{~h}$ synthesis time). With thicker brushes, there is an increasing steric repulsion exerted by the polymer brush on the antibodies ${ }^{234,}{ }^{235}$, resulting in a decreased antibody loading. Above $19 \mathrm{~nm}$, the fluorescence intensity then follows a parabolic trend; increasing by almost two-fold for the thickness of $62 \mathrm{~nm}$ ( $6 \mathrm{~h}$ synthesis time) and decreasing thenceforth to reach a minimum for the thickness of $102 \mathrm{~nm}$ ( $24 \mathrm{~h}$ synthesis time). Such variation was valid for all tested concentrations, being more pronounced at higher concentrations as intensity increases with concentration. The apparent inconsistency in the protein loading trend seemed to be contrary to several earlier reports which showed protein loading capacity consistently increasing with brush thickness $^{213}$, 214, 224, 232, 233 . However, the studies available from literatures were conducted through covalent attachment schemes, which is different from our case. Additionally, Xue et al. recently showed, through cell attachment studies, that the relationship between grafting density and protein adsorption on poly $(\mathrm{N}-$ isopropylacrylamide) (PNIPAM) at $\mathrm{T}>\mathrm{LCST}$ is also not as straightforward as expected $^{236}$. 


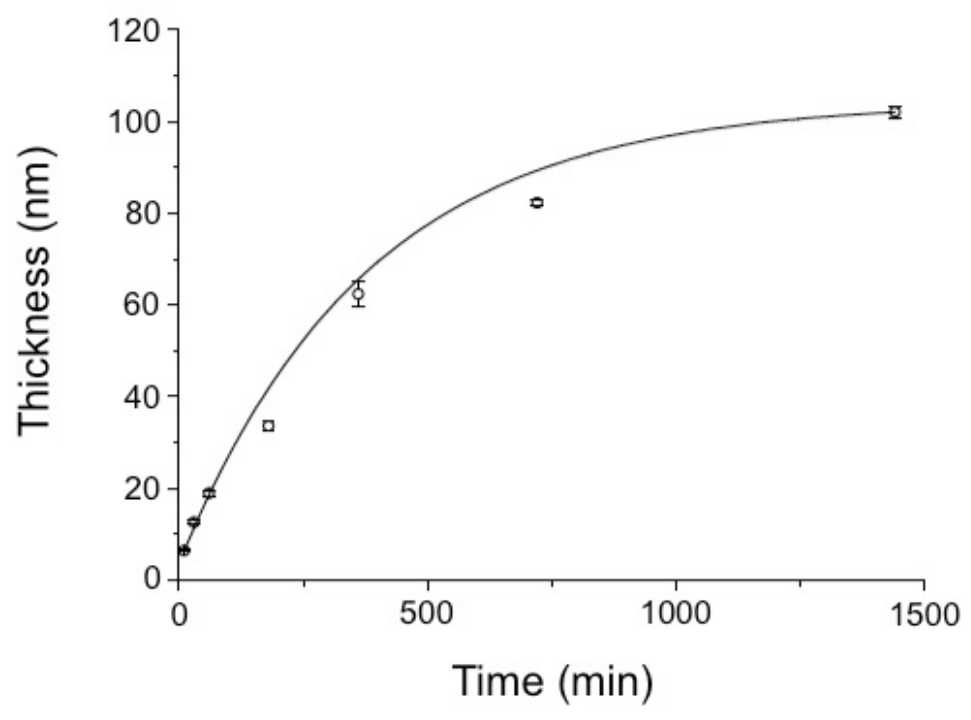

Figure 4-1. Ellipsometric thickness of polymer brush as a function of time. Error bars represent standard deviation $(n=5)$. (RSC $A d v$., 2013, 3, 9785 - Reproduced by permission of The Royal Society of Chemistry)

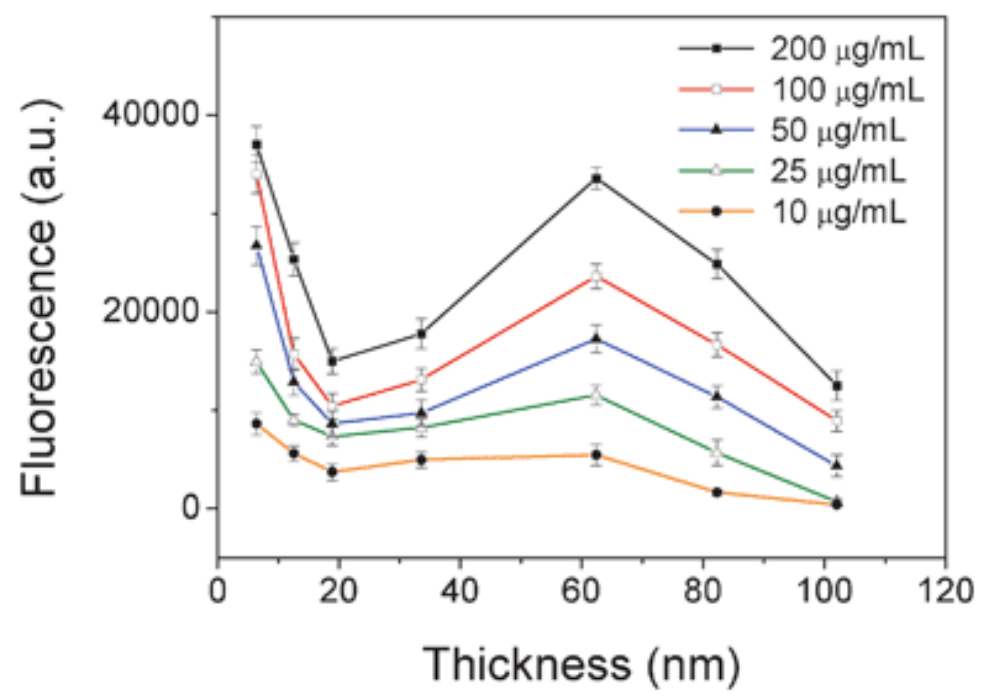

Figure 4-2. Fluorescence intensity of printed spots as a function of polymer brush thickness. The different point markers represent fluorescence arising from different IgG concentrations given in the figure legend. Error bars represent standard deviation ( $n=20)$. (RSC Adv., 2013, 3, 9785 - Reproduced by permission of The Royal Society of Chemistry) 
An investigation using tapping mode atomic force microscopy (TM-AFM) revealed that the surface morphology after physical immobilization of antibodies distinctly varied especially between brushes with thicknesses of 6, 19 and $62 \mathrm{~nm}$. Since pristine brushes grown at various thicknesses resulted in similar surface morphologies, a representative pristine brush grown to a thickness of $60 \mathrm{~nm}$ is presented in Figure 4-3A for reference. For the lowest POEGMA thickness attained (6 $\mathrm{nm}$ ), height retrace revealed $\mathrm{IgG}$ as closely-packed bumps protruding from a relatively smooth surface (Figure 4-3B). This is consistent with our expectation that the antibodies simply adsorb on top of the brush. For $19 \mathrm{~nm}$, the height retrace and 3D rendering revealed a smooth, mildly undulating surface (Figure 4-3C). While we initially expected little to no IgG present on the brush due to the reduction of nonspecific adsorption as brush thickness is increased, the phase retrace interestingly displayed a rough profile albeit the absence of obvious height variations, indicating the presence of antibodies beneath the polymer brush. Such profile is absent in the case of pristine POEGMA (Figure 4-4). Figure 4-2 also shows that the fluorescence intensity from a $19 \mathrm{~nm}$-thick brush only reduced to around half that of a $6 \mathrm{~nm}$-thick brush, instead of approaching zero, indicating that $\operatorname{IgG}$ could indeed still be effectively immobilized. Here, we believe compression of the underlying chains by $\operatorname{IgG}$ occurs as the brush can be perturbed ${ }^{235}$, and, since $19 \mathrm{~nm}$ is thicker than the longest dimension of $\operatorname{IgG}$, most of the surrounding brush chains are able to conceal the penetrated $\operatorname{IgG}$ when the brush is collapsed; giving the impression of infiltration underAFM. We shall refer this as 'pseudo-infiltration' in our following discussions. The fluorescent measurement (Figure 4-2) shows that the protein loading begins to rise above $19 \mathrm{~nm}$, implying that there are additional driving forces besides adsorption; otherwise the loading would continue to decrease with increasing brush thickness due to increasing 
steric repulsion of the brush polymer. Indeed, the height retraces for 62 and $102 \mathrm{~nm}$ thick brushes (Figures 4-3D \& E) revealed yet another morphology different from the first two cases. 3D rendering for the $102 \mathrm{~nm}$-thick brush revealed a rough and highly undulating surface (Figure 4-3E). Inferred by the fact that Figures 4-3B \& D indicated similar protein loading based on the fluorescence measurements, the difference in morphology suggest that at higher thicknesses, the antibodies do not adsorb in the same manner as on brushes with low thickness.

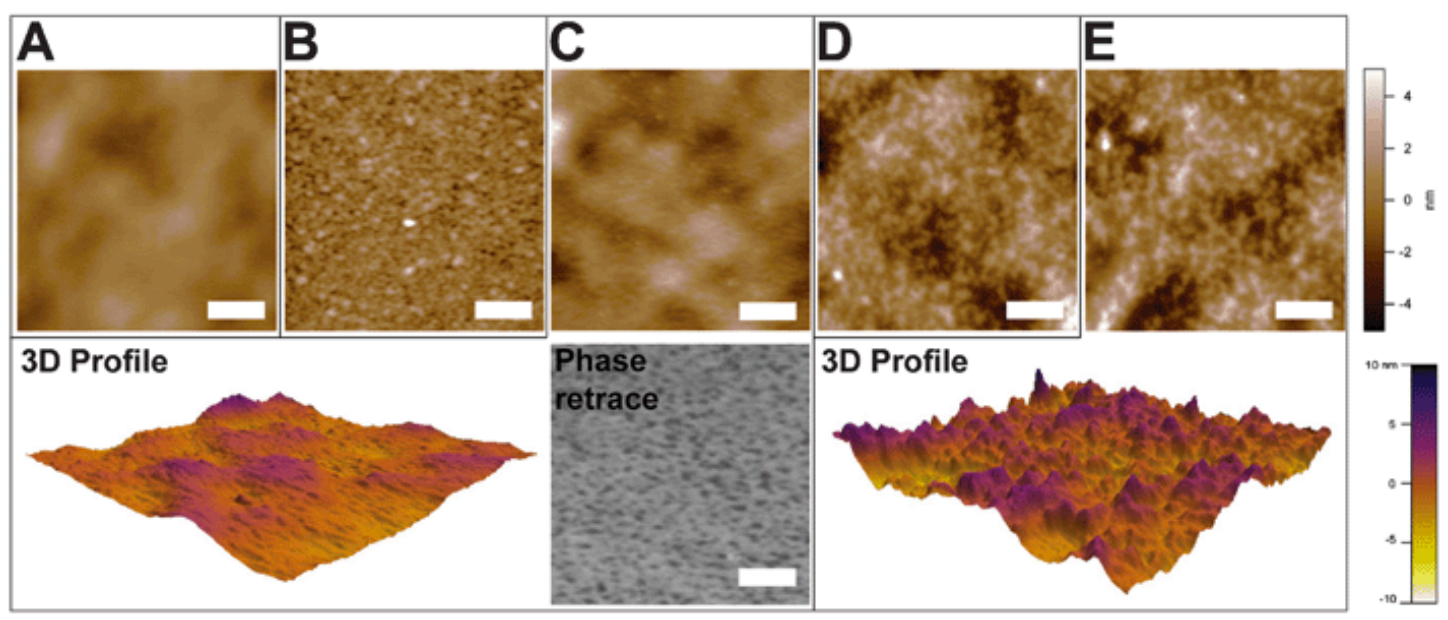

Figure 4-3. AFM height retrace of (A) a representative pristine POEGMA grown to a thickness of $\sim 60 \mathrm{~nm}$ for visual reference. AFM height retraces of POEGMA after physical immobilization of IgG via spot drying on (B) 6 nm-thick, (C) 19 nm-thick, (D) $62 \mathrm{~nm}$-thick and (E) $102 \mathrm{~nm}$-thick POEGMA. The phase retrace of $19 \mathrm{~nm}$-thick POEGMA with physically-immobilized IgG is included in (C) for reference. Threedimensional profiles of $19 \mathrm{~nm}$-thick and $102 \mathrm{~nm}$-thick POEGMA with physicallyimmobilized IgG are included in both $(\mathrm{C})$ and $(\mathrm{E})$ for comparison. The scan size for all retraces are $1 \mu \mathrm{m} 2$ with height range fixed at $10 \mathrm{~nm}$ for $2 \mathrm{D}$ images and $20 \mathrm{~nm}$ for $3 \mathrm{D}$ images. Scale bars represent $200 \mathrm{~nm}$. (RSC Adv., 2013, 3, 9785 - Reproduced by permission of The Royal Society of Chemistry) 


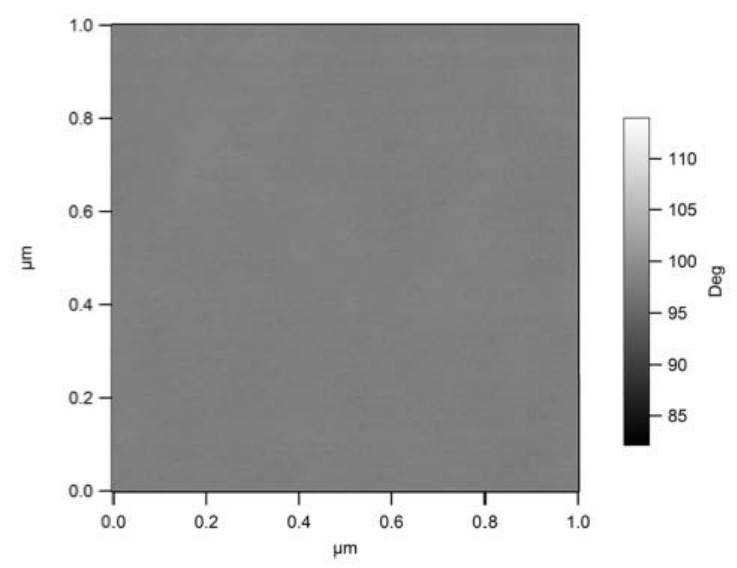

Figure 4-4. AFM phase retrace of a representative pristine POEGMA grown to a thickness of $\sim 60 \mathrm{~nm}$. (RSC Adv., 2013, 3, 9785 - Reproduced by permission of The Royal Society of Chemistry)

\subsubsection{Orientation of physically immobilized antibodies across different brush thickness}

While conventional attractive effects ${ }^{228-230}$ can explain the driving force behind physical antibody immobilization per se, it remains unclear why antibodies eventually organize within the brush in a particular way when thickness is varied. In order to gain a better understanding of the possible mechanism which governs the way physically immobilized proteins are arranged in polymer brushes of different thickness, we compared our observations with expressions derived in a theoretical model developed by Kim and O'Shaughnessy ${ }^{237}$. The model describes the ease of nanoinclusion penetration into a dry polymer brush by taking into account free energy penalties. The expressions used in the model provide brush fluctuation scales $\xi_{\text {blob }}$ and $\xi_{\text {surf }}$ as well as inclusion scales $b^{*}$ and $b_{\max }$. Briefly, they are derived based on the fact that polymer chains stretch to the free surface in a densely-grafted polymer brush system. In doing so, entropy produces fluctuations about the average path as the individual chains form a random coil structure. The scale of these fluctuations is the blob size $\xi_{\text {blob }}$ which is a 
function of distance from the grafting surface. The mean $\xi_{\text {blob }}^{-}$represents an average blob size of the brush interior. At the surface of the brush, the fluctuation is larger than

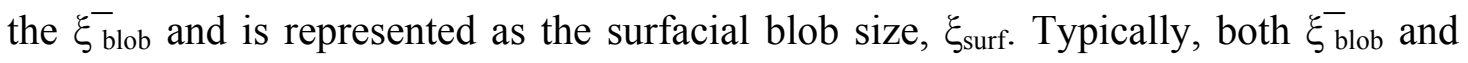
$\xi_{\text {surf }}$ would indicate whether the brush could accommodate a nanoinclusion of size b in the interior and on the surface, respectively. On the other hand the inclusion scales $b^{*}$ and $b_{\max }$ would determine how nanoinclusions distribute within the brush. If $b \ll b^{*}$, the distribution is even in the interior of the brush. If $b^{*} \ll b \ll b_{\max }$, the nanoinclusions only penetrate to a certain depth while for $b_{\max } \ll b$, they cannot penetrate the brush. As these expressions were derived based on a free energy minimization standpoint (i.e. a nanoinclusion denied of brush penetration would solely be due to a high free energy penalty), it is particularly relevant to our system as there are no chemical attachment schemes involved. Furthermore, the model takes into account the average fluctuations of individual chains when it forms a random coil structure, which is realistic since the molecular weight distribution across the substrate may vary after surface-initiated polymerization is completed.

The estimated size scales specific to our system is shown in Table 4-1 (detailed calculations are available in the APPENDIX). Since IgG ( $\sim 150 \mathrm{kDa})$ was reported to have approximate dimensions of $14.5 \mathrm{~nm} \times 8.5 \mathrm{~nm} \times 4.5 \mathrm{~nm}^{238-240}$ and Stokes' radius of $5.5 \mathrm{~nm}^{241}$, based on the latter, no penetration would occur because all values of $b_{\max }$ across various thicknesses are smaller than $11 \mathrm{~nm}$ (i.e. $2 \times$ Stokes' radius). This is in agreement with the observation that bulky antibody recognition occurs in the upper part of POEGMA even for the case involving covalent attachment schemes ${ }^{210}$. While this could intuitively explain the decrease in protein loading from 6 to $19 \mathrm{~nm}$ since there would be lower adsorption with thicker brushes, it could not explain the increase 
in loading from 19 to $62 \mathrm{~nm}$. We then proceeded our investigation by taking into account the structural geometry and dimensions of $\operatorname{IgG}$ against the variation of $\xi_{\text {surf }}$ and $b_{\max }$.

Table 4-1. A list of dry thickness of POEGMA as measured via ellipsometry along with the corresponding estimated size scales which are used in comparison with the dimensions of $\operatorname{IgG}$ to determine the ease of its penetration into the brush. Values for dry thickness and the respective size scales are rounded up to 3 significant figures (RSC Adv., 2013, 3, 9785 - Reproduced by permission of The Royal Society of Chemistry)

\begin{tabular}{cccccc}
\hline $\begin{array}{l}\text { Synthesis } \\
\text { time (min) }\end{array}$ & $\begin{array}{l}\text { Dry thickness } \\
(\mathrm{nm})\end{array}$ & $\begin{array}{l}\bar{\xi}_{\text {blob }} \\
(\mathrm{nm})\end{array}$ & $\begin{array}{l}\xi_{\text {surf }} \\
(\mathrm{nm})\end{array}$ & $\begin{array}{l}b^{*} \\
(\mathrm{~nm})\end{array}$ & $\begin{array}{l}b_{\max } \\
(\mathrm{nm})\end{array}$ \\
\hline 10 & 6.40 & 2.05 & 2.21 & 0.60 & 2.33 \\
30 & 12.6 & 2.05 & 2.77 & 0.60 & 2.76 \\
60 & 18.9 & 2.05 & 3.18 & 0.60 & 3.06 \\
180 & 33.6 & 2.05 & 3.85 & 0.60 & 3.53 \\
360 & 62.4 & 2.05 & 4.73 & 0.60 & 4.12 \\
720 & 82.3 & 2.05 & 5.19 & 0.60 & 4.42 \\
1440 & 102 & 2.05 & 5.57 & 0.60 & 4.66
\end{tabular}

We found that, generally in the range of 19 to $102 \mathrm{~nm}$, the values of $\xi_{\text {surf }}$ and bmax are relatively close to the thickness of each arm of the antibody, which is $4.5 \mathrm{~nm}$. It is also theoretically predicted that vertical orientation and slender shape gives the lowest free energy while a circular plate shape is the worst shape with highest energy for penetration $^{237}$. We therefore hypothesize that, while it is highly unlikely that the entire antibody infiltrates into the brush, it is possible for one arm of the Y-shaped molecule (either of the $\mathrm{F}_{\mathrm{ab}}$ or $\mathrm{F}_{\mathrm{c}}$ region) to be embedded between adjacent polymer chains. Hence at this point, we postulate that physical-immobilization occurs based on two major modes across increasing thicknesses (Figure 4-5); adsorption on the surface of 
the brush and embedment at the sub-surface of the brush. Adsorption can be further broken down into two minor modes; simple adsorption and pseudo-infiltration. When thickness is increased, adsorption occurs with decreasing probability. From 19 to 62 $n m$, loading contribution via embedment starts to occur when the values of $\xi_{\text {surf }}$ and $b_{\max }$ approaches the thickness of each arm of the antibody. At $62 \mathrm{~nm}$, maximum protein loading arises from an optimal combination of both adsorption and embedment. However, with increasing thickness, the contribution from adsorption begins to deplete. This eventually caused loading to decrease for thickness above 62 $\mathrm{nm}$. On top of that, it is also possible that the reduction in IgG embedment efficiency when $\xi_{\text {surf }} \gg$ b contributes to reduced protein loading.

\section{Physical Embedment}

\section{Adsorption}

\section{Simple Adsorption}

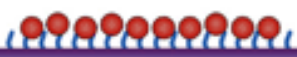

Pseudo-Infiltration

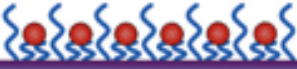

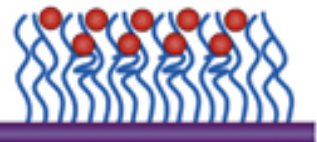

Figure 4-5. A simplified illustration of the postulated contributing modes of physicalimmobilization of antibodies via spot-drying across increasing polymer brush thickness (from left to right). Note that while the schematics depict the brush as straight chains for simplicity, in reality they exist as random coils. (RSC Adv., 2013, 3, 9785 - Reproduced by permission of The Royal Society of Chemistry)

To confirm that the antibodies are indeed anchored firmly to the polymer brush via the physical immobilization approach, we performed a simple experiment by subjecting the printed substrates to prolonged washing (60 min) on an orbital shaker. Severe tailing was observed for substrates grown with 6 nm-thick POEGMA after washing (Figure 4-6A), indicating that the antibodies are not spatially confined. This 
is not surprising since they are only adsorbed on the surface with random configurations. Tailing effects were less severe for brushes grown with thicknesses of 13 and $19 \mathrm{~nm}$ (Figures 4-6B \& C), which is due to the decreased protein loading observed by fluorescence measurements. For brushes with thickness $34 \mathrm{~nm}$ and above, absolutely no tailing effects were observed (Figures 4-6D-G). This supports our hypothesis that, as the brush gets thicker, embedment becomes the dominating factor. Note that while $6 \mathrm{~nm}$ and $62 \mathrm{~nm}$-thick brushes gave similar fluorescence intensities, implying little difference in protein loading, the fact that severe tailing occurred in the former but was absolutely absent in the latter strongly indicated the presence of spatial constriction due to embedment. In retrospect, this showed that, while a thin brush could potentially give highest transient protein loading, protein retention is poor due to simple adsorption. Hence, in our case, the brush thickness which allowed optimal protein loading with highest capacity and retention capability was found in the brush thickness of $\sim 62 \mathrm{~nm}$. This study indicates that the embedment mode plays a crucial role in achieving strong physical immobilization, suggesting the feasibility of physical immobilization for the application in the various substrate-based protein assays. 


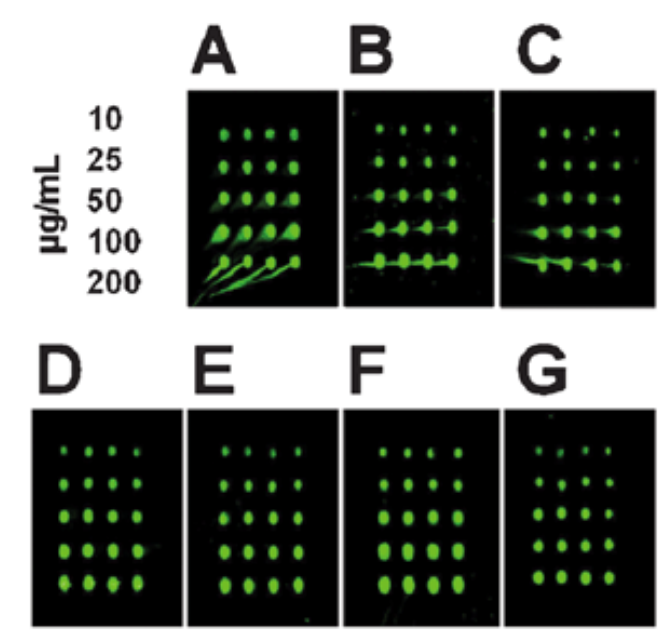

Figure 4-6. Fluorescence scanned images of the print- and dried spots after being rinsed, dried and further subjected to prolonged washing for $1 \mathrm{~h}$ on the orbital shaker at $120 \mathrm{rpm}$. The images are obtained from 6, 13, 19, 34, 62, 82 and $102 \mathrm{~nm}$-thick POEGMA (A-G). (RSC Adv., 2013, 3, 9785 - Reproduced by permission of The Royal Society of Chemistry)

After establishing that physically-immobilized IgG are embedded at the subsurface of relatively thick brush polymers, we were motivated to know the extent of this embedment. Such study would provide an understanding of how embedment results in good protein retention and ultimately explain the stability of physical immobilization. Typical height retraces which scan a homogeneous area can never provide sufficient evidence since AFM only scans the top-most layer. To achieve this more accurately, we scanned a droplet edge area and obtained a cross-sectional height profile (Figure 4-7A). The overall profile is also shown in 3D (Figure 4-7B). Generally, the height variations do not exceed $2 \mathrm{~nm}$. Also, from the 3D perspective, it is clear that the spotted region with physically-immobilized IgG generally lies in the same plane as the unspotted region; there is no obvious height difference between these two regions. This provided additional evidence that while infiltration was impermissible, adsorption did not occur either; if not height variations should be at 
least $\sim 4 \mathrm{~nm}$ on average (in the case of IgG resting flat on the surface). Based on this, we confirm that $\operatorname{IgG}$ are embedded at the top portion of the brush standing, with one arm penetrating between adjacent polymer brush chains (Figure 4-7C). The height variations not exceeding $2 \mathrm{~nm}$ suggest that a small fraction of the non-penetrating chains $\sim 1-2 \mathrm{~nm}$ rests over the top of the brush. We believe such oriented configuration exposes the antigen-binding regions, allowing easy access for target antigens during detection. This may explain the excellent performance of antibody microarrays which employed physical immobilization. At the same time, since most of the IgG body lies inside the brush, it is well-protected from the outside environment and not as susceptible to protein denaturation, resulting in longer shelf-life. 


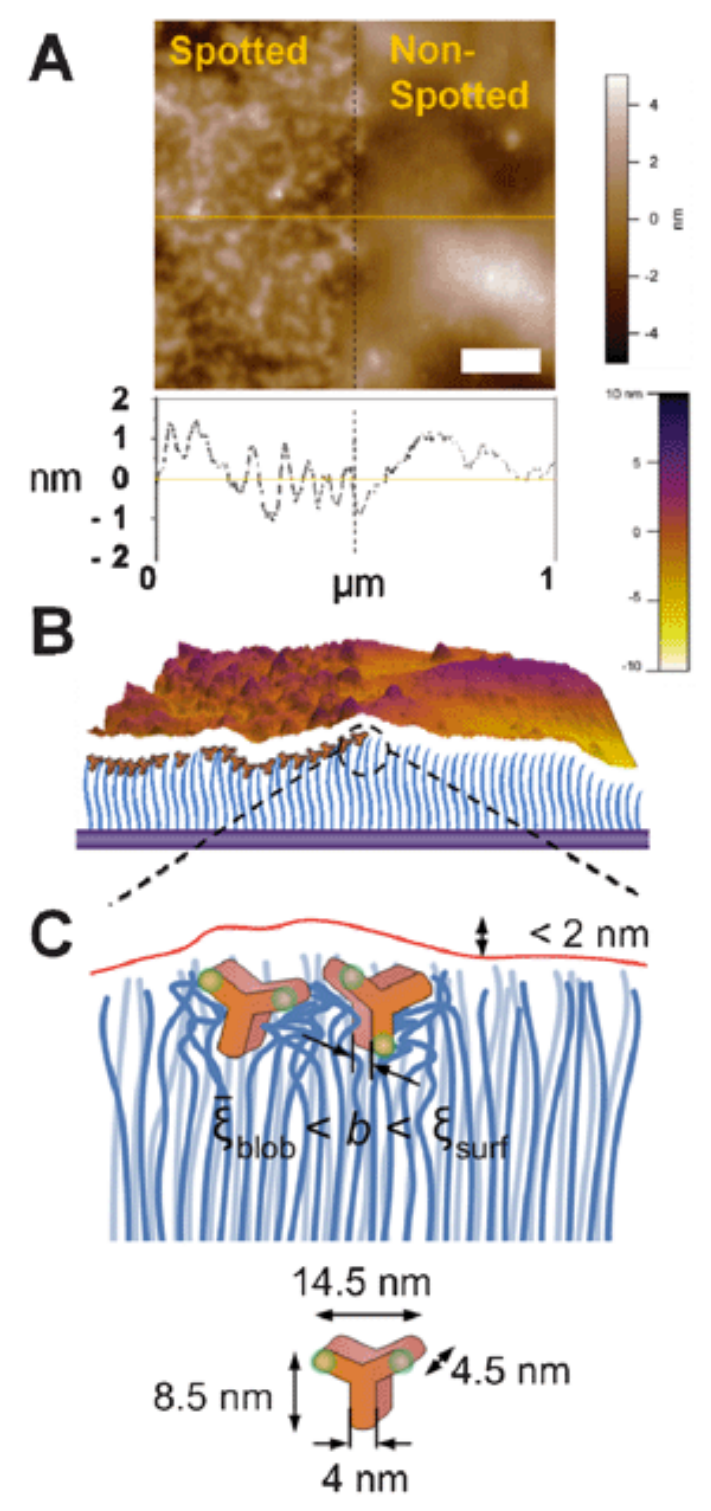

Figure 4-7. (A) AFM height retrace at the spot edge region of IgG physicallyimmobilized via spot drying on $100 \mathrm{~nm}$-thick POEGMA with the corresponding crosssectional height profile along the central region as indicated by the gold line. (B) The corresponding 3D profile of (A) with a representative schematic illustrating the arrangement and relative position of IgG on the brush. (C) A zoom-in view of the schematic in (B) illustrating the configuration of embedded IgG as well as the probable line of AFM height retrace (red line). The schematics are roughly drawn to scale. (RSC Adv., 2013, 3, 9785 - Reproduced by permission of The Royal Society of Chemistry) 


\subsubsection{Biomolecular assay based on physically immobilized antibodies}

At this stage, we have no concrete evidence to ascertain which arms (either $\mathrm{F}_{\mathrm{ab}}$ or $F_{c}$ region) of the antibody are more preferable in being embedded into the brush. However, in any case, it would still result in at least one accessible $\mathrm{F}_{\mathrm{ab}}$ region since each antibody molecule has two $\mathrm{F}_{\mathrm{ab}}$ arms. When the glass slides with spotted antibodies are immersed in a target solution, we believe both the tight fit between the penetrating chain of IgG and the adjacent polymer brush chains as well as the effective secondary interactions due to close proximity ensure good retention without compromising target binding efficiency. At the same time, the surrounding polymer chains which are not bound to IgG would still be able to provide excellent non-fouling property since they are not chemically modified in any way. To demonstrate the excellent operability of the brush polymer, a biomolecular assay was performed based on arrays of physically-immobilized monoclonal mouse anti-human IgG using a brush thickness of $\sim 62 \mathrm{~nm}$. Our results showed a limit-of-detection of $100 \mathrm{fg} \mathrm{mL}^{-1}$ with a dynamic range of $100 \mathrm{fg} \mathrm{mL}^{-1}$ to $10 \mathrm{ng} \mathrm{mL}^{-1}$, in agreement with the performance results obtained by an earlier work which uses a brush thickness of $100 \mathrm{~nm}^{227}$. This shows that a thick brush may not always be necessary to achieve a good detection performance since optimized conditions may be met at a much lower brush thickness. The dose-response curve for the detection is depicted in Figure 4-8A. From the microarray image shown in Figure 4-8B, there is no fluorescence spots appearing from the 'control' array confirming no non-specific adsorption of AlexaFluor 546-goat anti-human IgG detection antibody (a comparison of non-specific adsorption on nonPOEGMA treated glass is shown in Figure 4-9). Also, no fluorescence was observed from rows spotted with a blank solution of PBS bufferregardless of the concentration of human IgG introduced. For rows spotted with bovine serum albumin (BSA), 
fluorescence only appeared at the highest concentration of $10 \mathrm{ng} \mathrm{mL} \mathrm{L}^{-1}$ human $\mathrm{IgG}$. This means that at lower concentrations of human $\operatorname{IgG}$, no non-specific binding occurred between human IgG and the spotted BSA. Conversely, this highlights the conservation of the capture antibody's activity within the POEGMA brush since it is able to capture human $\operatorname{IgG}$ at concentrations as low as $1 \mathrm{fg} \mathrm{mL}^{-1}$ and still produce an observable fluorescence signal.

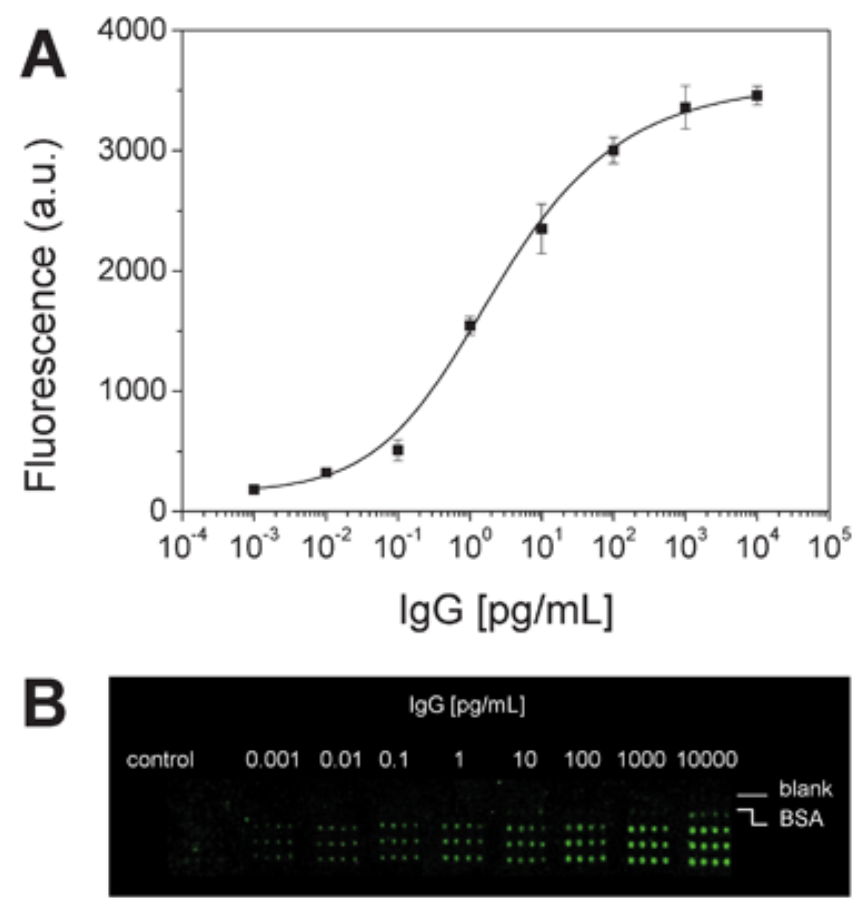

Figure 4-8. (A) Dose-response curve for an IgG microarray detecting human $\operatorname{IgG}$ in PBS buffer over a concentration range of $1 \mathrm{fg} \mathrm{mL}^{-1}$ to $10 \mathrm{ng} \mathrm{mL} \mathrm{m}^{-1}$ using a brush thickness of $\sim 62 \mathrm{~nm}$ and (B) an image of a typical microarray. The control array in the far left represents a printed array which have been incubated with blank PBS buffer and subsequently incubated with AlexaFluor 546-goat anti-human IgG. The rows labeled 'blank' and 'BSA' represent spots which have been printed with blank PBS buffer solution and a solution of bovine serum albumin in PBS, respectively. (RSC $A d v ., 2013,3,9785$ - Reproduced by permission of The Royal Society of Chemistry) 


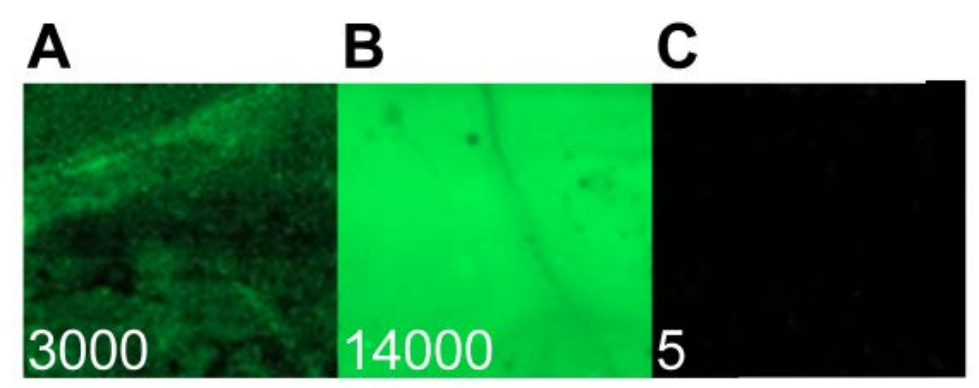

Figure 4-9. A comparison of fluorescence intensities of an adsorbed layer of AlexaFluor 546-goat anti-human IgG on (A) bare, (B) aminopropyltriethoxysilaneand (C) POEGMA-treated glass substrates. The substrates were fully immersed in a solution of $1 \mu \mathrm{g} / \mathrm{mL}$ AlexaFluor 546-goat anti-human IgG in PBS buffer. The numbers at the bottom left of each scan represents the average fluorescence signal intensities expressed in arbitrary units. (RSC $A d v$., 2013, 3, 9785 - Reproduced by permission of The Royal Society of Chemistry)

\subsubsection{Stages involved in the physical immobilization of antibodies}

In summary, the seemingly contradictory physical immobilization of proteins on non-fouling polymer brushes can be explained by considering the following cases. In a fully wet state, osmotic pressure is low and the proteins can be easily expulsed by the pressure exerted by the extending brush chains. At this stage, secondary bonding interactions are not applicable or not significant since the proteins are not in close proximity to the brush chains for a prolonged period. This explains why in a wet state, the brush can effectively reduce non-specific adsorption. During drying, osmotic pressure increases and this causes the proteins to approach the surface of the brush. At this stage, we explain in this work that proteins can either be simply adsorbed, pseudoinfiltrated or embedded depending on the brush thickness. Secondary bonding interactions start to become more relevant as throughout the drying process, more proteins will experience these attractive, secondary, non-covalent forces since they are in close proximity, if not, in direct contact with the brush for a longer period. After 
being fully dried, these forces of interactions play the role of retaining the proteins during washing in whatever arrangement they may be in (i.e. simply adsorbed on the brush, pseudo-filtrated in the brush or embedded on the brush). However, since different arrangements result in different proximities between the proteins and the brush chains when the brush is re-swollen in solution, proteins which are not in close proximity or not in direct contact with the brush chains (e.g. when there is no tight fit between the arm size of IgG and the surface blob size) will be more easily expulsed during the first rinse since they experience weaker secondary interactions. This, in turn, determines the final loading density.

\subsubsection{Role of simple aggregation in physical immobilization of antibodies}

While an argument may arise in question of the role of simple aggregation and/or denaturation in physical immobilization, we have shown through our experimental evidences that if we were to be contented with such a straightforward scenario, and apply it universally across the entire thickness variation (i.e. from 6 to $100 \mathrm{~nm}$ ), we are faced with several inconsistencies. Firstly, AFM images show that the surface morphology of $62 \mathrm{~nm}$ and $100 \mathrm{~nm}$ are similar. If indeed physical immobilization is largely due to denatured/aggregated proteins binding on surface defects or accessible areas we expect larger aggregates in thicker brushes combined with the decreasing probability of attachment of these aggregates upon increasing brush thickness. Consequently, there should be a significant difference in the surface coverage/morphology since fluorescence signal decreased to half. Specifically, for such a scenario we would expect to see, via AFM, globules with increasing sizes as thickness increases, with the amount of these large globules decreasing with increasing thickness. However, we show through Figure 4-3 that this was not the case. Secondly, our protein retention studies via prolonged washing revealed that tailing only occurred 
for the cases of 6,13 and $19 \mathrm{~nm}$ brush thickness. If attachment is solely via the binding of aggregates for all cases from 6 to $100 \mathrm{~nm}$ brush thickness, with decreasing probability of attachment of these aggregates upon increasing brush thickness, then we would have also observed tailing effects upon prolonged washing for higher thicknesses as what we observed for the case of $6 \mathrm{~nm}$ (in fact we would have observed more severe tailing effects for cases of higher thicknesses since large globules would have increasing probability of detachment). In contrast, we observed from Figure 4-6 that for higher thickness, absolutely no tailing occurred, signifying a difference in binding mode between low- and high-thickness brushes. Finally, we have shown that we could detect human $\mathrm{IgG}$ down to a concentration of $1 \mathrm{fg} \mathrm{mL}^{-1}$, at a thickness which gave the highest loading, signifying a high level of activity and implying the irrelevant effects of denaturation. Based on the above arguments, while we cannot deny that there could be some degree of aggregation/denaturation, we could not claim that aggregation/denaturation is a main contributor or role-player for immobilization of IgG as aggregation/denaturation alone could not be used to explain, beyond any reasonable doubt, the overall trend observed in this work.

Finally, while our approach has specifically rejected the notion of IgG infiltration in POEGMA, we were interested to see if the same approach could also be applied to support non-antibody protein infiltration claimed by others in order to extend its validity to smaller proteins. The report by Gautrot et al. was selected because they utilized the same polymer brush system and had substantial experimental evidence to support their claim of His-GFP infiltration into $30 \mathrm{~nm}$-thick POEGMA ${ }^{213}$. Now, by referring to our estimated size scales in Table 1 , at $\sim 30 \mathrm{~nm}, \xi_{\text {blob }}^{-}=2.05$ while $b_{\text {max }}=$ 3.53. Looking at the size of GFP, which is approximately a cylinder of $2 \mathrm{~nm}$ diameter by $4 \mathrm{~nm}$ height, infiltration would occur since, if we take $\mathrm{b}$ to be the shorter 
dimension, $\mathrm{b}^{*} \ll \mathrm{b} \ll \mathrm{b}_{\max }$. This means that GFP would penetrate, with a vertical orientation, to a certain depth within the brush interior as $b<\xi_{\text {blob. Such deduction is }}^{-}$ consistent with their reported results, confirming the reliability of our empirical interpretation.

\subsection{Conclusion}

We presented evidence to show that the organization of physically-immobilized antibodies in polymer brushes differ across a wide range of thicknesses by correlating our experimental evidences with a reported theoretical model. While adsorption is dominant at relatively low thickness, we concluded that at higher thicknesses, IgG could not infiltrate deeply into POEGMA but are in fact embedded at the sub-surface of the brush. This revealed that increasing brush thickness may not always directly correlate to an increased loading capacity. In our system that employs IgG antibody and POEGMA brush polymer, the optimal loading capacity was achieved at the brush thickness of $\sim 62 \mathrm{~nm}$, much less than the maximum thickness attainable. Our work have particularly highlighted the importance of size factors in governing the organization of physically-immobilized proteins in polymer brushes and demonstrated that a systematic approach is essential to predict maximum protein loading. This would eventually allow higher efficiency control over the physical immobilization process in the future, which is especially relevant to the advancement of probe immobilization strategies in protein biochip technologies. For instance, by knowing the size of proteins, the most effective brush thickness at a fixed grafting density could be calculated to achieve maximum protein loading. More interestingly, the control over brush parameters could also be exploited to physically orientate proteins which has distinctive shapes. As we foresee vast prospect in the physical immobilization 
methodology due to its extreme simplicity, our contribution hopes to provide a starting tool which would encourage its use in future works. 


\section{Chapter 5}

\section{Gold Nanoparticle Incorporation in POEGMA via Infiltration and Its Application in Solid-Phase Colorimetric Sensing of Lead}

\subsection{Introduction}

Many nanoparticle-based colorimetric detection methods for biological and chemical analytes have been developed in the solution-phase ${ }^{114,242-247}$. Although the nanoparticle-based colorimetric detection allows straightforward observation through simple readout schemes often with high sensitivity and specificity, solution-phase colorimetric sensors in general still have several limitations which practically deter its widespread use ${ }^{113,248}$. First, while they permit detection via unaided eyes for analytes in clear solutions, it would be difficult to discern color hue or intensity changes when examining colored samples and complex mixtures, such as blood and food samples. Second, nanoparticles (e.g., gold nanoparticles, AuNPs) in solution are vulnerable to aggregation; thereby reducing the shelf life of the sensors. While transforming the detection from solution-phase to solid-phase may alleviate these issues, most immobilization approaches require some form of modification on the nanoparticle and/or substrate ${ }^{249-252}$. Such rigid chemical immobilization strategy would no longer permit the spatial reorganization of nanoparticles, which represents the primary characteristic of most of current colorimetric assays based on nanoparticle aggregation.

Therefore, to realize the full potential of nanoparticle-based colorimetric detection on a solid-phase platform and utilize it for the development of practical nanosensing 
devices, it is desired to (1) immobilize the nanoparticles while still allowing a certain degree of "mobility", (2) adopt an immobilization strategy that does not involve any chemical modification so that the nanoparticle can be modified downstream according to the requirements of any detection scheme, and (3) attain optimized nanoparticle loading, while ensuring good molecular access to the nanoparticles.

In this contribution, we address these issues by loading as-prepared AuNPs into surface-grown, unmodified poly(oligo(ethylene glycol)methacrylate) (POEGMA) brushes $^{227}$. POEGMA was chosen primarily because of the ability of its polyethylene glycol groups to displace citrate molecules ${ }^{253}$. Since the side branches of POEGMA are abundant with ethylene glycol (EG) groups, the attachment of citrate-capped AuNPs to the side chains of the brush is feasible without the need for any chemical modification. POEGMA has also been established to possess nonfouling property allowing detection from complex fluids (e.g., blood) without nonspecific interferences. We hypothesize that small AuNPs can maintain sufficient mobility within pliable polymer brushes, while they can easily form 3D assembly via infiltration; hence, in the present study, we employed AuNPs with an average size of $4.2 \mathrm{~nm}$ which can effectively attain 3D assemblies even with brushes grown as thin as $18.6 \mathrm{~nm}$. Additionally the ability to precisely control the brush thickness allows for optimal AuNP loading within a 3D matrix; compared to the AuNP monolayer used in current chip-sensors, the large loading capacity offered by the 3D matrix can greatly enhance the colorimetric intensity. Moreover, the fact that POEGMA can be grown on clear glass implies that any form of colorimetric quantification can be easily performed in the same manner as in AuNP solution (e.g., via UV-vis absorbance, transmittance, and reflectance), with minimal interference from the substrate; unlike paper-based 
colorimetric assays which requires scanning followed by the use of an image processing software for quantification of color intensities ${ }^{254-256}$.

\subsection{Specific Methods}

\subsubsection{Nanoparticle incorporation into POEGMA}

The glass coverslips that had been earlier modified with POEGMA were subsequently immersed in $3 \mathrm{~mL}$ of AuNP $(\sim 4 \mathrm{~nm}$ in diameter) solution and left overnight. Upon removal, they were copiously rinsed with deionized $\mathrm{H}_{2} \mathrm{O}$. They were then rinsed 3 times in deionized $\mathrm{H}_{2} \mathrm{O}$ under orbital shaking at $120 \mathrm{rpm}$ for 2 min each and finally blown dry with $\mathrm{N}_{2}$.

\subsubsection{Nanoparticle postfunctionalization and lead detection}

The concentrations and $\mathrm{pH}$ of reagents were fixed according to conditions optimized in solution $^{242}$. The reaction durations was set based on a new study which was specific to our system (i.e., solid-phase platform using AuNPs of a different size). The reaction times for both postfunctionalization and detection were eventually set to 45 min. For postfunctionalization, glass coverslips with AuNP-incorporated POEGMA were first immersed in a freshly prepared $3 \mathrm{~mL}$ of a solution of $1 \mathrm{mM}$ sodium thiosulfate in $5 \mathrm{mM}$ glycine- $\mathrm{NaOH}$ buffer at $\mathrm{pH} 10$ under gentle shaking for $45 \mathrm{~min}$. Upon removal, they were copiously rinsed with $5 \mathrm{mM}$ glycine- $\mathrm{NaOH}$ buffer and blown dry with $\mathrm{N}_{2}$. For lead detection in water, the postfunctionalized coverslips were subsequently immersed in a $3 \mathrm{~mL}$ solution containing varying concentrations of lead ions (ranging from $1.0 \mathrm{pM}$ to $100 \mu \mathrm{M})$ and $1 \mathrm{mM}$ 2-mercaptoethanol (2ME) at $\mathrm{pH} 10$. Upon removal, they were copiously rinsed with $5 \mathrm{mM}$ glycine- $\mathrm{NaOH}$ buffer $(\mathrm{pH} 10)$ and blown dry with $\mathrm{N}_{2}$. Lead detection in human serum was performed by diluting the human serum 5-fold with a solution of $1 \mathrm{mM}$ 2-mercaptoethanol (2ME) at $\mathrm{pH} 10$ and 
spiked with lead ions over a concentration range of 0.1-100 nM. Upon removal, they were copiously rinsed with $5 \mathrm{mM}$ glycine- $\mathrm{NaOH}$ buffer $(\mathrm{pH} 10)$ followed by DI water and blown dry with $\mathrm{N}_{2}$.

\subsubsection{Characterization}

Polymer brush thickness measurements were obtained from silicon substrates by ellipsometry in air using a J.A. Woolam $\alpha$-SE spectroscopic ellipsometer. UV-vis spectra were obtained using a Shimadzu UV-2540 UV spectrophotometer. Atomic Force Microscopy data were obtained in air from an Asylum Research MFP-3D AFM system conducted in tapping mode. Scanning electron micrographs were obtained from a JEOL JSM-6700F FESEM microscope. All samples were coated with Pt for 30 $\mathrm{s}$ at $20 \mathrm{~mA}$. Dynamic light scattering (DLS) measurements were obtained from a Malvern Instruments Nano ZS Zetasizer equipped with a DTS Nano Software. Transmission electron micrographs were obtained from a JEOL JEM-1200EX TEM microscope with a tungsten filament at an accelerating voltage of 100-120 kV. X-ray photoelectron spectroscopy (XPS) measurements were obtained using a (UHV) VG ESCALAB 220I-XL system with a monochromatic A1 Ka X-ray source.

\subsection{Results and Discussion}

\subsubsection{Gold nanoparticle loading as a function of polymer brush thickness}

We first investigated the effect of varying the thickness of POEGMA on AuNP loading via simple immersion before assessing the performance of the sensing platform. The POEGMA brushes were grown on glass coverslips via surface-initiated atomic transfer radical polymerization up to a maximum thickness of $\sim 100 \mathrm{~nm}$ (Figure 5-1). The chemical composition of POEGMA was confirmed via X-ray photoelectron spectroscopy (XPS) (Figure 5-1A) before they are grown up to a 
maximum thickness of $\sim 100 \mathrm{~nm}$ (Figure 5-1B). The peak binding energies from the XPS detailed scan of the carbon C1s region were found at $284.8 \mathrm{eV}, \sim 286.5 \mathrm{eV}$ and $\sim 289 \mathrm{eV}$, corresponding to $\mathrm{C}-\mathrm{C}, \mathrm{C}-\mathrm{O}$ and $\mathrm{C}=\mathrm{O}$ chemical states, respectively. The peak corresponding to $\mathrm{C}-\mathrm{O}$ is the highest followed by $\mathrm{C}-\mathrm{C}$, confirming the presence abundant ethylene glycol side chains of POEGMA, since in every ethylene oxide repeat unit there is almost twice -C-O- compared to -C-C- bonds. Additional -C-Cbonds also originate from the methacrylate backbone. The $\mathrm{C}=\mathrm{O}$ chemical state contribute to the lowest peak since it is only present as a single unit of ester linkage between the methacrylate backbone and the side chain for each monomer added. Coverslips treated with aminopropyltriethoxysilane (APTS) were included alongside POEGMA-coated ones to serve as a control, all of which were subsequently subjected to overnight immersion in a solution of as-prepared, citrate-capped AuNPs (Figure 52). The citrate-capped AuNPs have zeta-potential of $\sim-30 \mathrm{mV}$. We obtained uniform AuNP loading across different substrates and synthesis batches ( $\mathrm{n} \geq 30$, Figure 5-3).
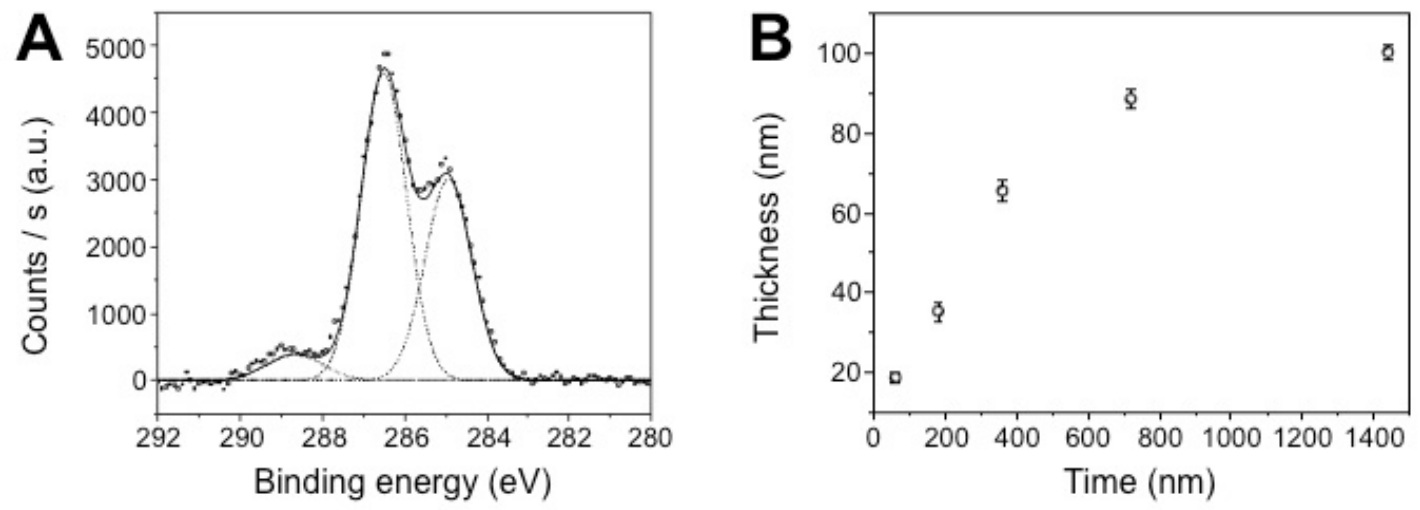

Figure 5-1. (A) XPS spectra of as prepared 1 h-POEGMA, (B) ellipsometric POEGMA thickness variations with respect to synthesis time. Fig. (B) Reprinted with permission from Anal. Chem., 2013, 85 (8), pp 4094-4099. Copyright 2013 American Chemical Society. 

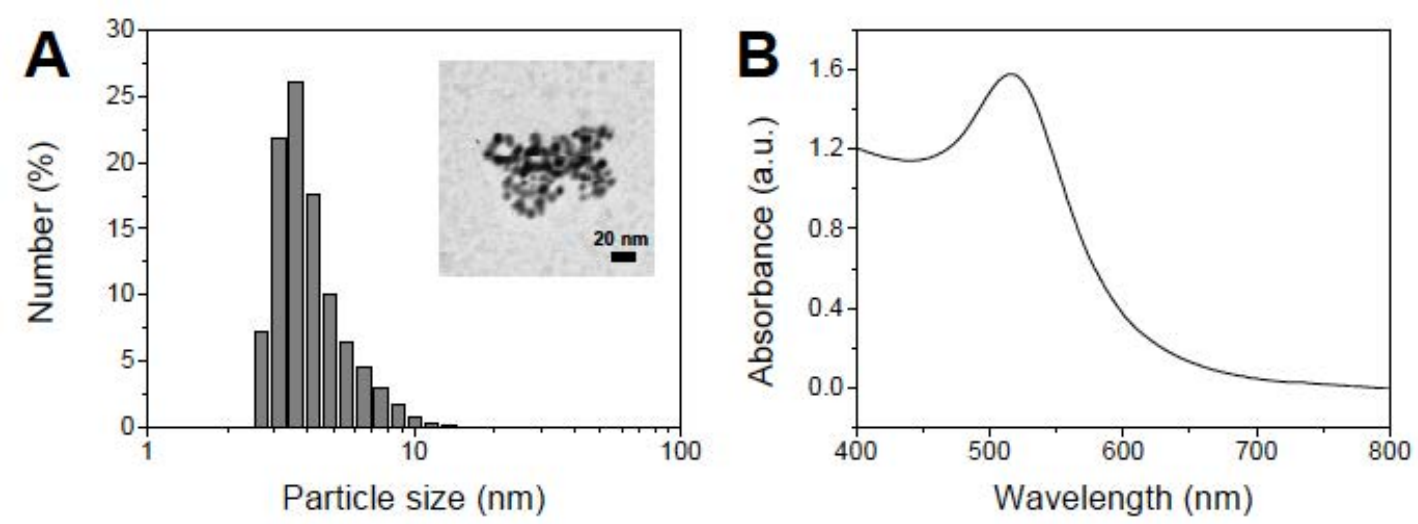

Figure 5-2. (A) Size distribution of small-diameter AuNPs used in this work as measured via dynamic light scattering. The mean size is determined to be $4.2 \mathrm{~nm}$. Inset show a TEM image of the AuNP sample. (B) UV-vis spectra of as-prepared solution of AuNP with an absorbance peak at $517 \mathrm{~nm}$. Reprinted with permission from Anal. Chem., 2013, 85 (8), pp 4094-4099. Copyright 2013 American Chemical Society. 

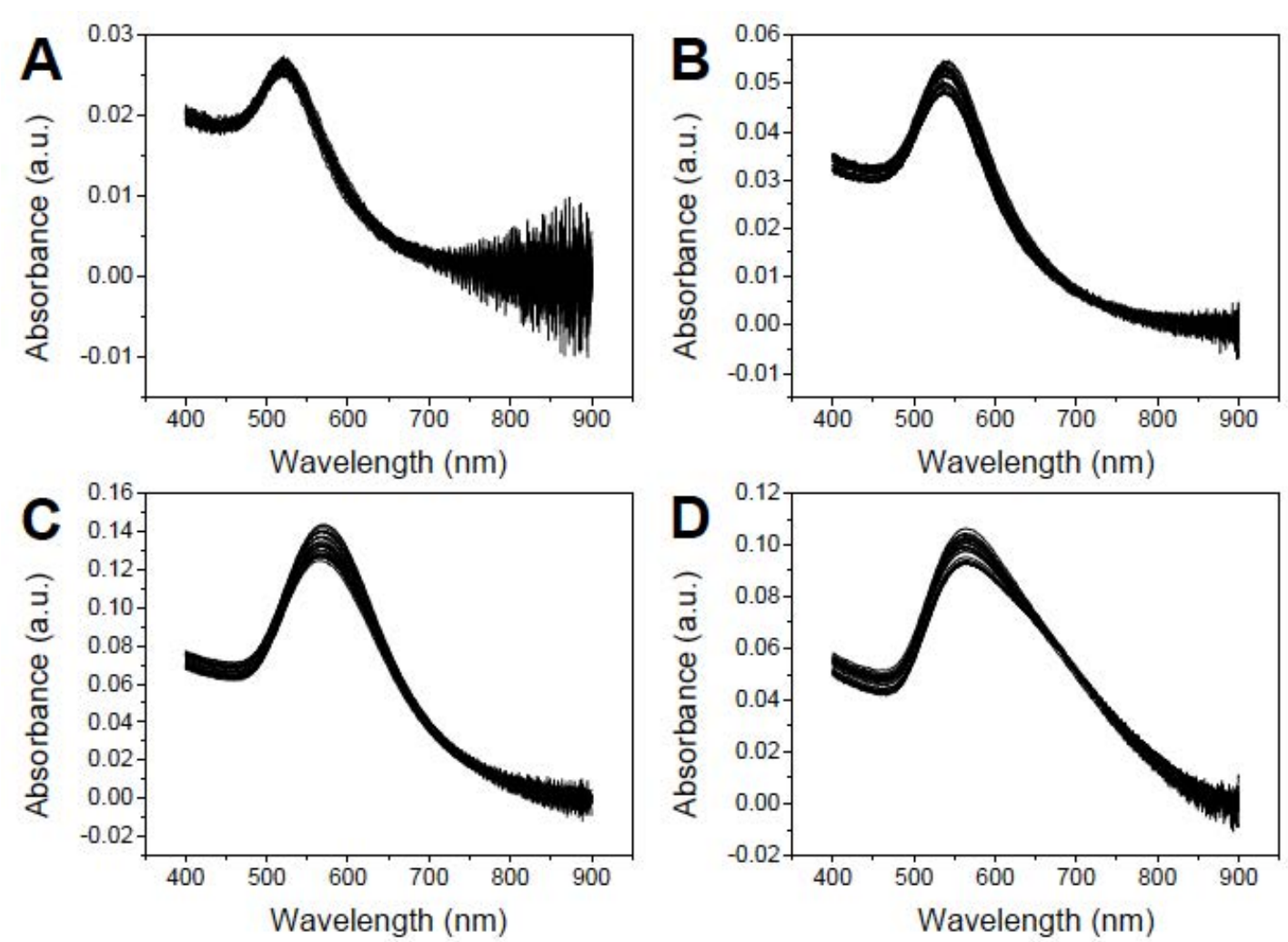

Figure 5-3. UV-vis spectra from a set of 30 samples of as-prepared (A) APTS-treated, (B) 1 h-POEGMA, (C) 3 h-POEGMA and (D) 6 h-POEGMA obtained from 3 separate synthesis batches of 10 samples each. Reprinted with permission from Anal. Chem., 2013, 85 (8), pp 4094-4099. Copyright 2013 American Chemical Society.

The averaged UV-vis absorbance spectra of the AuNP-loaded POEGMA substrates are shown in Figure 5-4A. While the control sample treated with APTS yielded lowest absorbance due to monolayered AuNPs, samples with POEGMA grown for $1 \mathrm{~h}$ (1 h-POEGMA) showed approximately 2-fold increased loading. The absorbance peak red-shifted $\sim 12 \mathrm{~nm}$ indicating a more closely packed AuNP assembly. The loading increased more significantly with the POEGMA grown for $3 \mathrm{~h}$ (3 h-POEGMA) with its peak absorbance being 5 times larger than the control. A peak shift of $\sim 43 \mathrm{~nm}$ was also observed. Contrary to the expected trend, the POEGMA grown for $6 \mathrm{~h}$ (6 h-POEGMA) yielded a decreased AuNP absorbance relative to $3 \mathrm{~h}$ POEGMA, accompanied by a slight peak broadening; the full-width at half-maximum 
of $3 \mathrm{~h}$ - and $6 \mathrm{~h}$-POEGMA spectra are 184 and $224 \mathrm{~nm}$, respectively. This is possibly due to the ability of the thicker brush to displace more citrate groups on the AuNPs leading to AuNP aggregation after maximum loading is reached ${ }^{257}$.
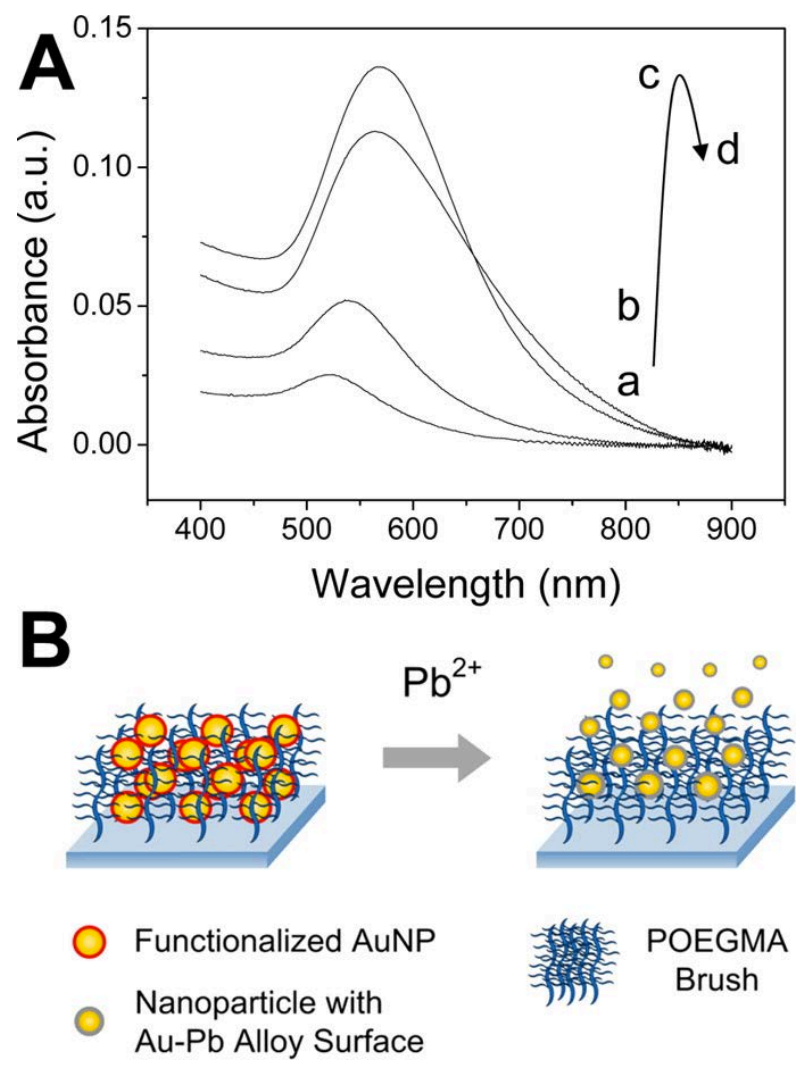

Figure 5-4. (A) UV-vis absorbance spectra showing AuNP loading variation for APTS-treated substrate (a), 1 h-POEGMA (b), 3 h-POEGMA (c), and 6 h-POEGMA (d). (B) Detection pathway showing the formation of nanoparticles with $\mathrm{Au}-\mathrm{Pb}$ alloy surface upon exposure of thiosulfate-functionalized AuNPs to lead ions and their subsequent release from the polymer brush matrix. Reprinted with permission from Anal. Chem., 2013, 85 (8), pp 4094-4099. Copyright 2013 American Chemical Society.

\subsubsection{Lead ion detection from water}

The AuNP-POEGMA system was used to detect lead, which has been recognized as a major environmental pollutant due to its high toxicity ${ }^{258}$. The detection chemistry 
relying on the leaching of AuNPs ${ }^{242}$ was adopted and the resultant sensing platform is shown in Figure 5-4B. First, AuNPs immobilized in the POEGMA are functionalized with thiosulfate (i.e., through thiosulfate adsorption on AuNP surface). This step is crucial to ensure that the leaching of gold by 2-mercaptoethanol (2ME) is mediated, allowing $\mathrm{Pb} 2+$ to act as an effective catalyst; without thiosulfate functionalization, gold will be easily leached by $2 \mathrm{ME}$ since it is a strong leachant. The thiosulfatefunctionalized AuNPs dissolve in the presence of $2 \mathrm{ME}$ and lead(II) ions to form $\mathrm{Au}-$ $\mathrm{Pb}$ alloy on AuNP surface, as well as soluble $\mathrm{Au}^{+}-2 \mathrm{ME}$ complexes. The leached AuNPs covered by $\mathrm{Au}-\mathrm{Pb}$ alloy on their surface no longer experience strong interactions with the EG chains of the brush, releasing them from the brush matrix into the solution (Figure 5-5). This AuNP release from POEGMA matrix ultimately results in a decrease in UV-vis absorbance and color fading of the AuNP-POEGMA coated glass coverslip. This detection scheme was adopted primarily because such chemistry has been reported to detect lead with high sensitivity and specificity in solution ${ }^{242}$. Hence, the performance in solution would serve as a good basis of comparison for our solid-phase system. In addition, as opposed to the detection conducted in solution, whereby thiosulfate and $2 \mathrm{ME}$ are mixed with the target and AuNPs in a single step, we could herein isolate the thiosulfate adsorption step. This indicates the ability of preloaded AuNPs to be postfunctionalized within the polymer brush according to other detection schemes of interest. 


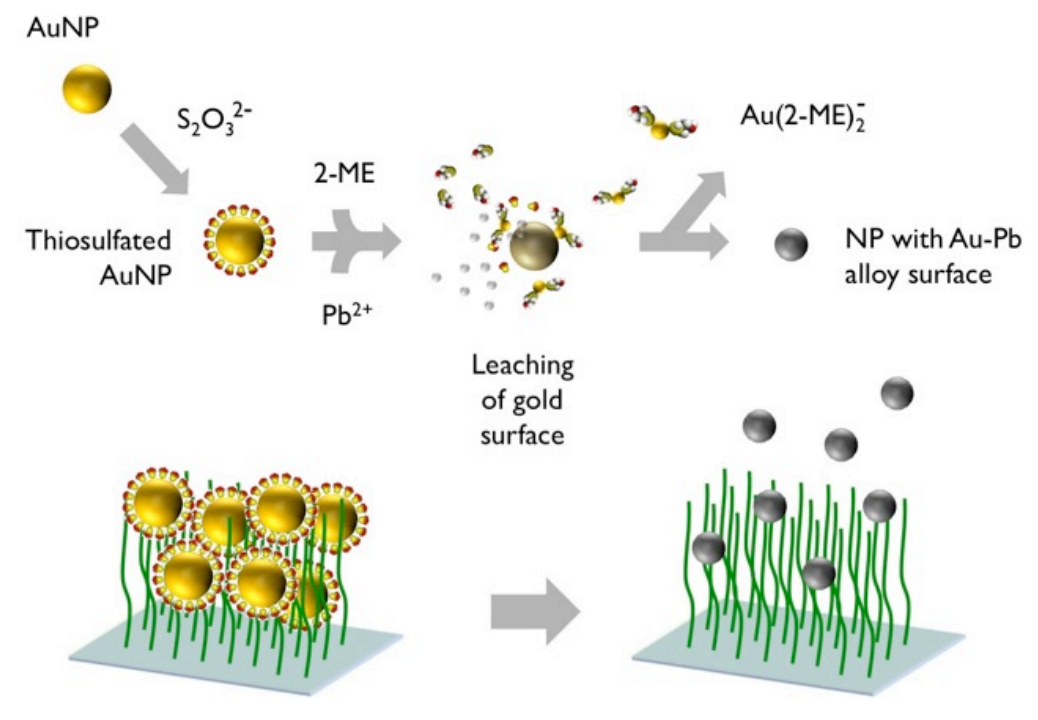

Figure 5-5. A more detailed description of the process of nanoparticle release from the polymer brush matrix. The functionalization with thiosulfate ensures that the gold leaching by 2-mercaptoethanol (2-ME) to form $\mathrm{Au}(2-\mathrm{ME})_{2}{ }^{+}$is mediated, allowing $\mathrm{Pb}^{2+}$ to be an effective catalyst in the process.

The averaged UV-vis spectra before and after in situ functionalization with thiosulfate (i.e., via adsorption) are shown in Figure 5-6. We confirmed successful thiosulfate functionalization for all the AuNP-POEGMA coated coverslips. We also checked that it was uniform across all the substrates (Figure 5-7). To study the detection performance, the substrates of AuNPs in POEGMA were first immersed in water containing $\mathrm{Pb}^{2+}$ ranging from $1.00 \mathrm{pM}$ to $100 \mu \mathrm{M}$ in the presence of $2 \mathrm{ME}$ and the responses were calculated based on absorbance drop (Figures 5-8A-D). The respective limit-of-detections (LOD) and dynamic ranges are tabulated in Table 5-1. Compared to monolayered AuNPs on APTS-treated substrates, 1 h-POEGMA yielded a much more gradual decrease in absorbance with respect to decreasing concentration of $\mathrm{Pb}^{2+}$ down to $10 \mathrm{pM}$. The vast improvement in the LOD $(25 \mathrm{pM})$ and dynamic range (100 pM to $100 \mathrm{nM}$ ) can be attributed to the fact that the decrease in absorbance of AuNP-POEGMA substrates is not solely a function of leaching, but also heavily 
relies on the release of AuNPs from the brush matrix. When we performed our detection using the same AuNPs in solution, we only managed to attain an LOD of 1 $\mathrm{nM}$ and dynamic range of 1-100 nM (Figure 5-9). To confirm the ability of AuNPs to be released from the polymer brush matrix, we performed an investigation with AuNPs within the POEGMA which are not protected with thiosulfate. Upon addition of blank solution of $2 \mathrm{ME}$, the sample is stripped and the release of AuNPs became noticeable when the solution acquired a mild tinge of pink at the region above the sample surface. This coloration eventually diffused evenly throughout the solution. Here the thiol group of $2 \mathrm{ME}$ strongly displaces the multidentate interaction between the EG groups of the brush and the gold surface allowing the release of the AuNPs. When the solution is retrieved and checked for its UV spectra, an absorbance peak around $520 \mathrm{~nm}$ is clearly present (Figure 5-10). In contrast, for nanoparticles which have been functionalized with thiosulfate, no coloration developed in the solution. The UV spectra from the solution also did not indicate any detectable absorbance peak, suggesting minimal release of AuNPs from the brush, if any. The formation of $\mathrm{Au}-\mathrm{Pb}$ alloy on the surface has also been confirmed via X-ray photoelectron spectroscopy (XPS) (Figure 5-11). We believe that this "release mechanism" is primarily responsible for a more mediated absorbance drop at low concentrations; thereby extending the linear range downward and reducing the LOD. 

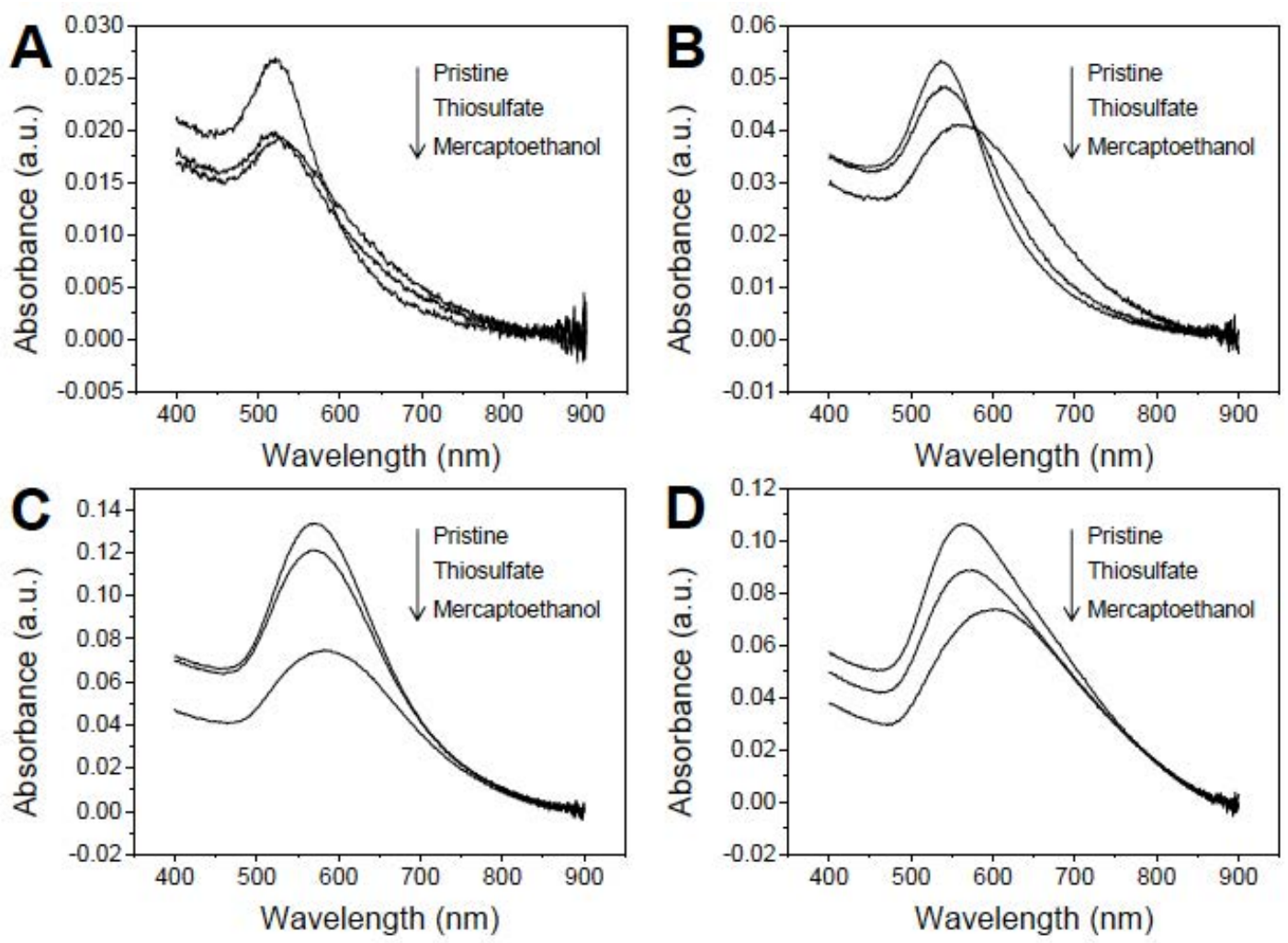

Figure 5-6. UV-vis spectra of as-prepared samples, after thiosulfate functionalization and after 2ME addition of (A) APTS-treated, (B) 1 h-POEGMA, (C) 3 h-POEGMA and (D) 6 h-POEGMA. Reprinted with permission from Anal. Chem., 2013, 85 (8), pp 4094-4099. Copyright 2013 American Chemical Society. 

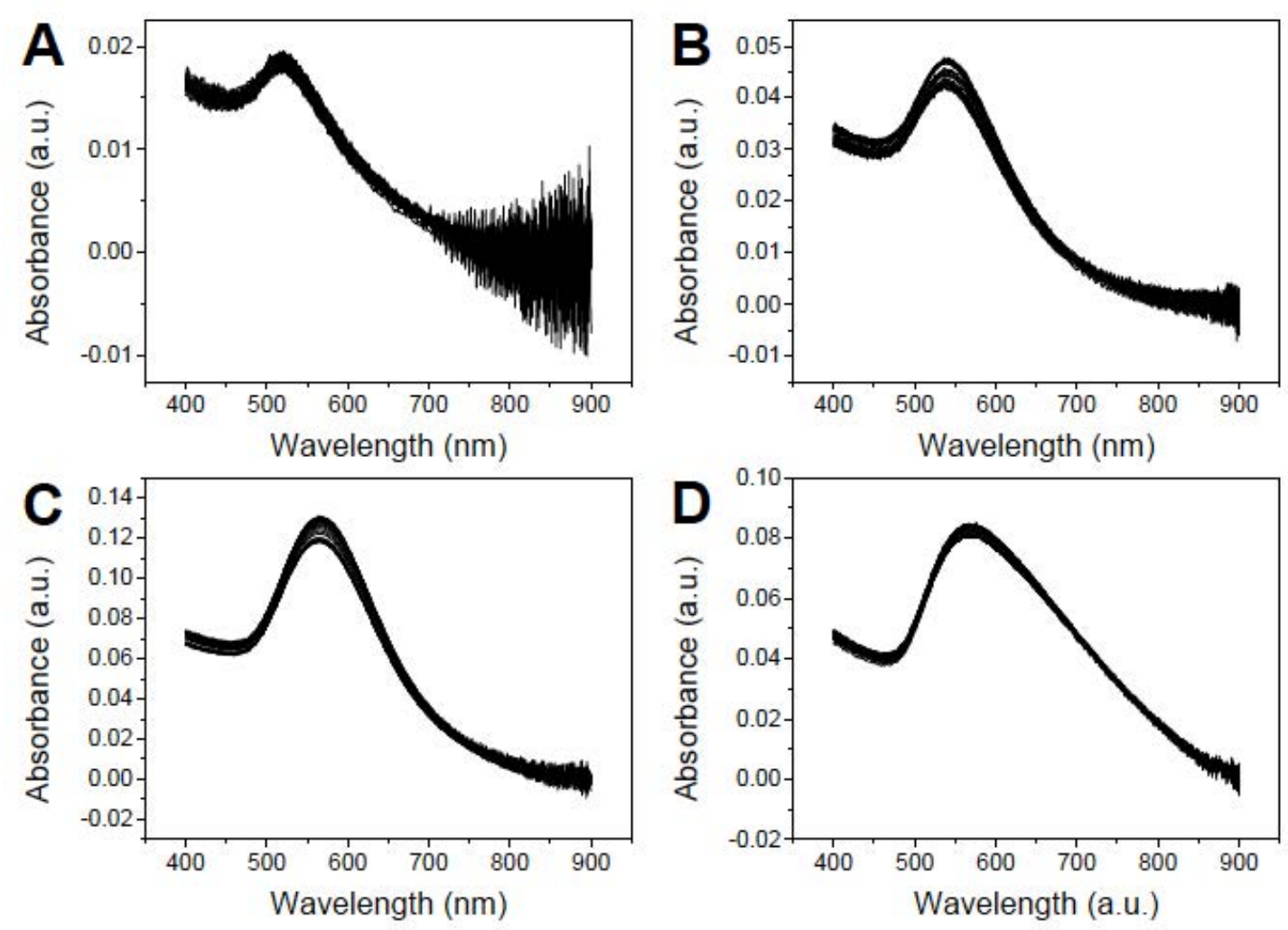

Figure 5-7. UV-vis spectra from a set of 30 samples of (A) APTS-treated, (B) 1 hPOEGMA, (C) 3 h-POEGMA and (D) 6 h-POEGMA obtained from 3 separate synthesis batches of 10 samples each, after thiosulfate functionalization. Reprinted with permission from Anal. Chem., 2013, 85 (8), pp 4094-4099. Copyright 2013 American Chemical Society. 


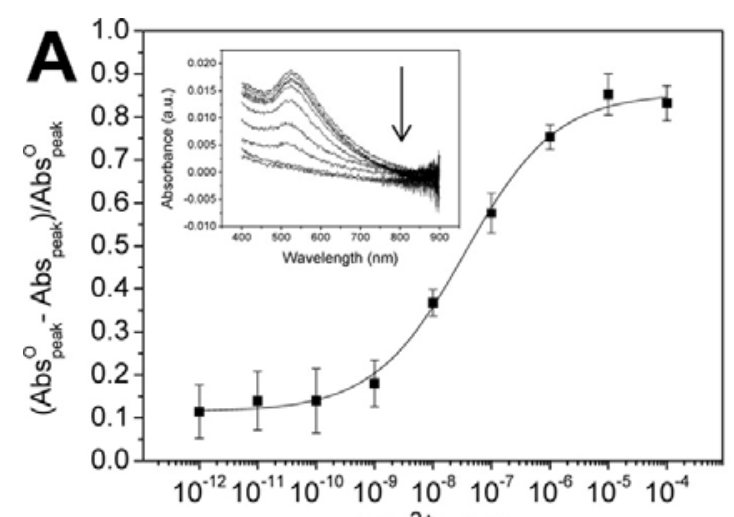

$\left[\mathrm{Pb}^{2+}\right](\mathrm{M})$

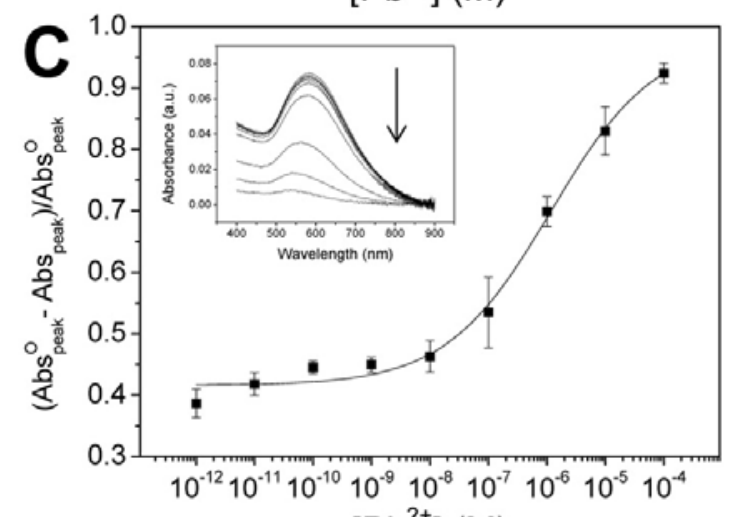

$\left[\mathrm{Pb}^{2+}\right](\mathrm{M})$

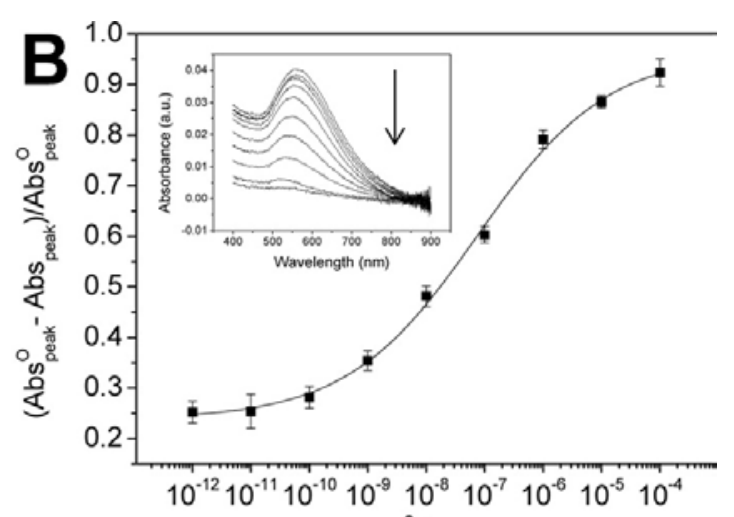

$\left[\mathrm{Pb}^{2+}\right](\mathrm{M})$

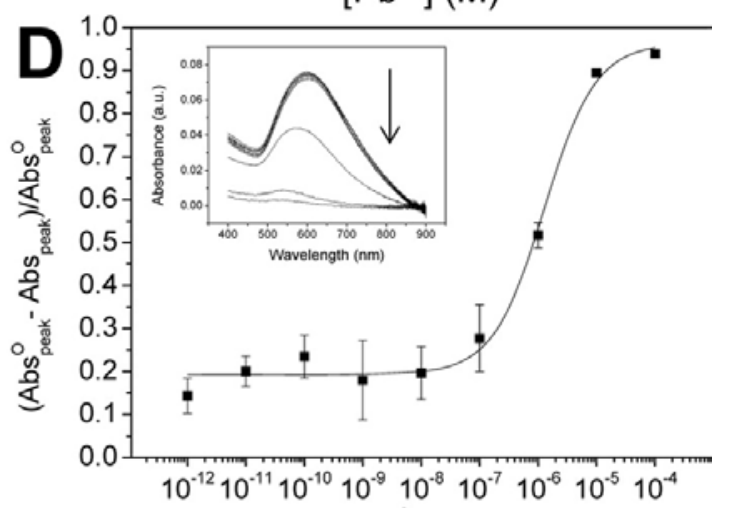

$\left[\mathrm{Pb}^{2+}\right](\mathrm{M})$

Figure 5-8. Responses with respect to $\mathrm{Pb}^{2+}$ concentrations ranging from $1 \mathrm{pM}$ to 100 $\mu \mathrm{M}$ for (A) APTS-treated substrates, (B) 1 h-POEGMA, (C) 3 h-POEGMA, and (D) 6 h-POEGMA. Insets show the respective spectra (arrow depicts increasing concentration). All error bars represent standard deviation $(n=3)$. Reprinted with permission from Anal. Chem., 2013, 85 (8), pp 4094-4099. Copyright 2013 American Chemical Society.

Table 5-1. Tabulated Limit-of-Detection (LOD), Dynamic Range, Dynamic Range Orders, and Associated $R^{2}$ Values for the Detection of $\mathrm{Pb}^{2+}$ Using the Various Substrates.

\begin{tabular}{lcccc} 
& $\begin{array}{c}\text { LOD }(S / N=3) \\
(\mathrm{nM})\end{array}$ & $\begin{array}{c}\text { dynamic range } \\
(\mathrm{DR})\end{array}$ & $\begin{array}{c}\mathrm{DR} \\
\text { orders }\end{array}$ & $\begin{array}{c}R^{2} \\
\text { value }\end{array}$ \\
\hline APTS & 1.7 & $10 \mathrm{nM}-1 \mu \mathrm{M}$ & 2 & 0.998 \\
1 h- & 0.025 & $0.1 \mathrm{nM}-0.1 \mu \mathrm{M}$ & 3 & 0.987 \\
$\quad$ POEGMA & & $10 \mathrm{nM}-100 \mu \mathrm{M}$ & 4 & 0.988 \\
$\begin{array}{l}\text { 3 h- } \\
\text { POEGMA }\end{array}$ & 8 & $100 \mathrm{nM}-10 \mu \mathrm{M}$ & 2 & 0.984 \\
$\begin{array}{l}\text { 6 h- } \\
\text { POEGMA }\end{array}$ & 42 & & &
\end{tabular}




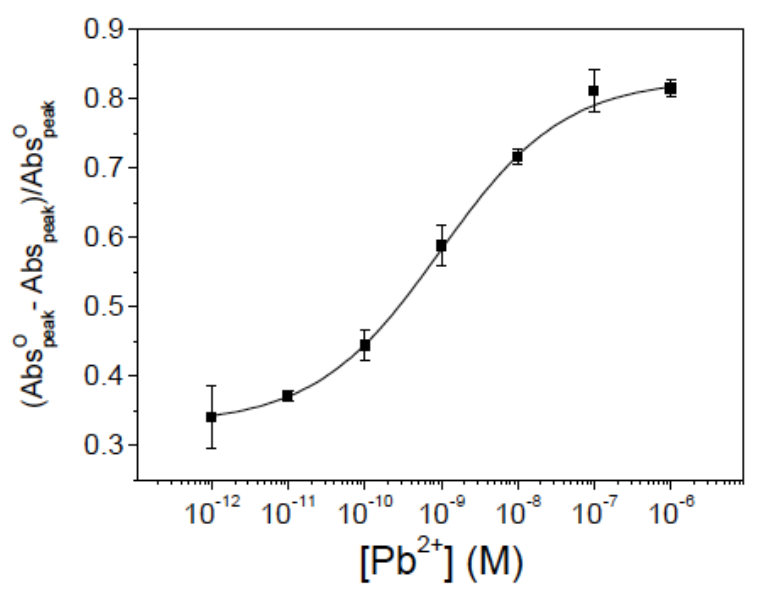

Figure 5-9. Solution phase responses with respect to $\mathrm{Pb}^{2+}$ concentrations ranging from $1 \mathrm{pM}$ to $1 \mu \mathrm{M}$. Error bars represent standard deviation $(n=3)$. Reprinted with permission from Anal. Chem., 2013, 85 (8), pp 4094-4099. Copyright 2013 American Chemical Society.

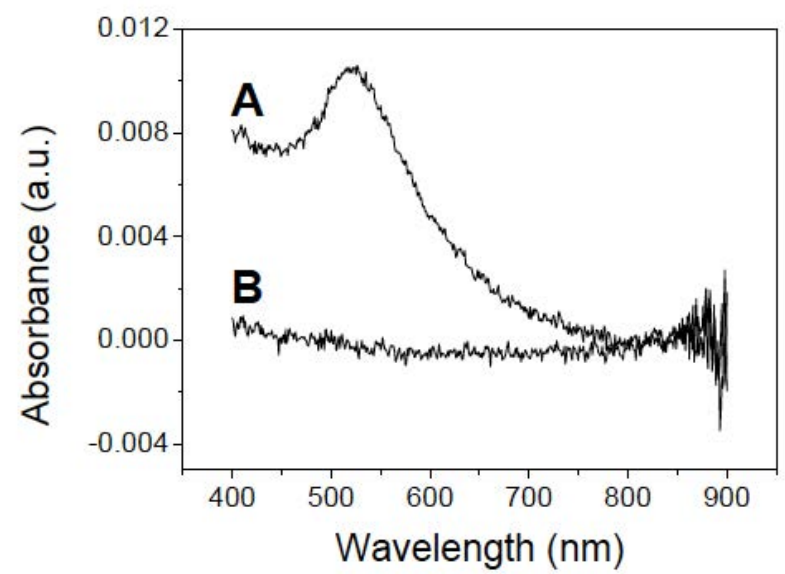

Figure 5-10. UV-vis spectra of retrieved $1.0 \mathrm{mM} 2 \mathrm{ME}$ solution ( $\mathrm{pH} 10)$ after immersion with (A) as-prepared AuNP-loaded 1 h-POEGMA substrate and (B) thiosulfate-protected AuNP loaded 1 h-POEGMA. Reprinted with permission from Anal. Chem., 2013, 85 (8), pp 4094-4099. Copyright 2013 American Chemical Society. 

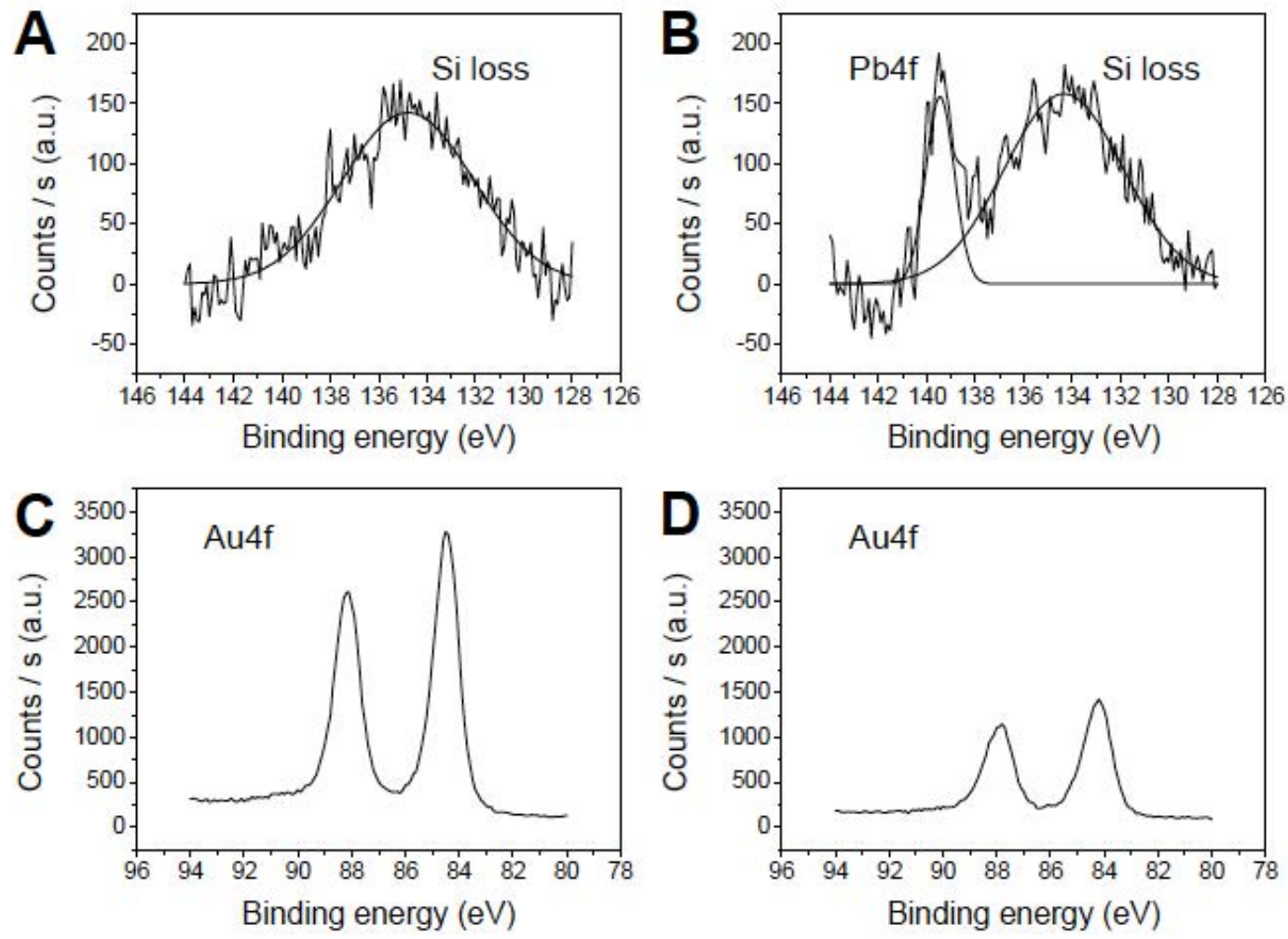

Figure 5-11. X-ray photoelectron spectroscopy (XPS) scans between Binding energies of 126-146 eV from 1 h-POEGMA substrates after detection of (A) $100 \mathrm{nM}$ and (B) 1 $\mu \mathrm{M} \mathrm{Pb}^{2+}$. Raw signal was baselined and curve-fitted using Origin Pro 8 software. A weak $\mathrm{Pb} 4 \mathrm{f}$ peak appeared in (B) on top of a common broad peak in both (A) and (B), which is possibly a Si plasmon loss peak. X-ray photoelectron spectroscopy (XPS) scans between Binding energies of 78-96 eV from 1 h-POEGMA substrates after detection of (A) $100 \mathrm{nM}$ and (B) $1 \mu \mathrm{M} \mathrm{Pb}^{2+}$. Reprinted with permission from Anal. Chem., 2013, 85 (8), pp 4094-4099. Copyright 2013 American Chemical Society.

\subsubsection{Effect of brush thickness on detection performance}

On the other hand, the release of AuNPs from the brush matrix is also dependent on the brush thickness; the thicker the brush, the higher the volume occupied by the brush and hence more difficult for the AuNPs to be released. Furthermore, thicker brushes would imply higher degree of multidentate interactions between AuNPs and EG groups in the polymer brush, which help retain the AuNPs. This explains why 
LOD and limit-of-linearity (LOL) generally shifts to higher concentrations for thicker brushes. Consequently, subsequent investigations were conducted using 1 h-POEGMA samples since it provided the lowest LOD and relatively good dynamic range.

The respective photographs of the AuNP-POEGMA samples after lead detection are shown in Figure 5-12. Compared to APTS-treated controls (Figure 5-12A), it is clear that POEGMA-coated samples (Figure 5-12B-D) allows better detection via unaided eyes due to a higher loading of AuNPs. In the case of $1 \mathrm{~h}$ - and $3 \mathrm{~h}$-POEGMA, gradual fading is observable across the detected concentration ranges. In clinical applications, the intensities can be matched against a reference card to determine the concentration of samples without the use of complex instrumentations. In the case of 6 h-POEGMA, the intensity drops drastically at $10 \mu \mathrm{M}$ due to its narrow dynamic range. Conversely, this implies that $6 \mathrm{~h}$-POEGMA possesses the highest response sensitivity across its dynamic range, which can be beneficial for a binary detection to simply test for the lead presence above a threshold concentration.

Field emission scanning electron micrographs (FESEM) of AuNP-POEGMA sample before and after target detection support our claim of nanoparticle release. Before detection (Figure 5-12E), AuNPs are densely embedded within the polymer brush matrix presenting a continuous composite morphology. After leached nanoparticles are released, some of unreacted AuNPs are observed as aggregates in and on the POEGMA (yellow circles in Figure 5-12F). 


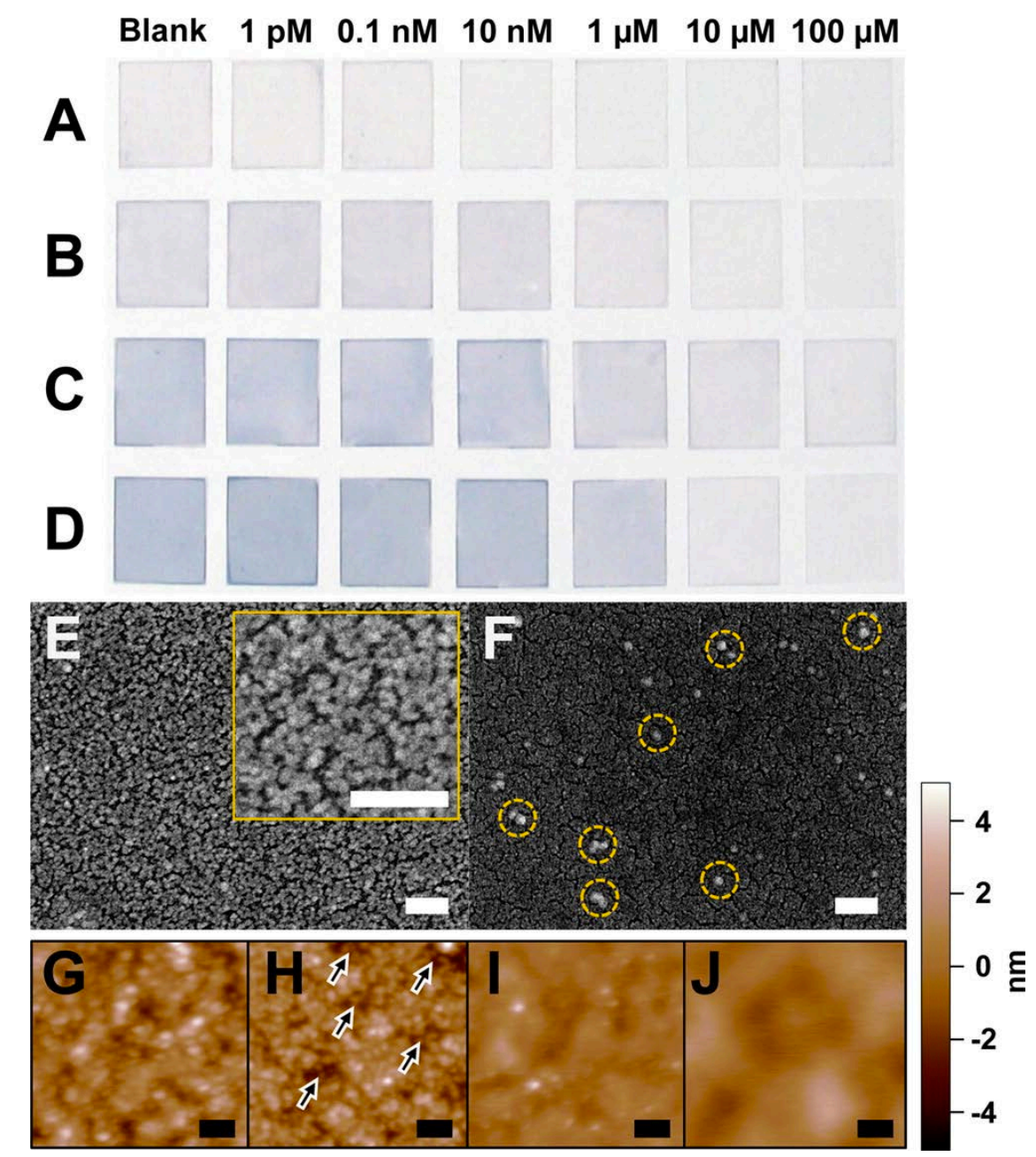

Figure 5-12. Photograph of (A) APTS-treated, (B) 1 h-POEGMA, (C) 3 h-POEGMA, and (D) 6 h-POEGMA glass coverslips after detection of $\mathrm{Pb}^{2+}$ concentrations ranging from blank, $1 \mathrm{pM}$ to $100 \mu \mathrm{M}$. FESEM images of 1 h-POEGMA substrate (E) before and (F) after detection of $100 \mu \mathrm{M} \mathrm{Pb} 2+$. Circled areas highlight the presence of some of the remaining AuNPs which appear aggregated. AFM height retraces of $1 \mathrm{~h}$ POEGMA after detection of $(\mathrm{G})$ blank, (H) $10 \mathrm{nM}$, and (I) $100 \mu \mathrm{M} \mathrm{Pb}^{2+}$. (J) AFM height retrace of pristine 1 h-POEGMA as reference. Arrows in Figure $3 \mathrm{H}$ highlight the presence of AuNPs at the base of ridge regions. All scale bars represent $100 \mathrm{~nm}$ with AFM height range of $10 \mathrm{~nm}$. Reprinted with permission from Anal. Chem., 2013, 85 (8), pp 4094-4099. Copyright 2013 American Chemical Society.

To confirm the presence of the POEGMA after lead detection, which cannot be discerned by SEM, we investigated the surface morphology of AuNP-POEGMA sample via atomic force microscopy (AFM) and compared samples after detection of 
blank, $10 \mathrm{nM}$ and $100 \mu \mathrm{M} \mathrm{Pb}^{2+}$. On the other hand, the AFM images back up our claim of AuNP release mechanism. After detection of blank, height retrace showed subtle height variations with few sharp bumps (white shades) arising from AuNPs, indicating closely packed AuNPs embedded in POEGMA (Figure 5-12G). After detection of $10 \mathrm{nM} \mathrm{Pb}^{2+}$, several regions showed sharper height variations or deep ridges (Figure 5-12H). The appearance of deep ridges implies the release of AuNPs. Small bumps also become more distinct, with a few located at the base of the ridges, indicating that AuNPs were 3-dimensionally assembled within the polymer brush via infiltration. After detection of $100 \mu \mathrm{M} \mathrm{Pb}^{2+}$, the height retrace showed that most of the smaller bumps have disappeared, implying that most of the AuNPs have been released (Figure 5-12I). The resultant cloudy appearance confirms that POEGMA is still present after target detection as evidenced via comparison with a reference sample bearing pristine POEGMA (Figure 5-12J).

\subsubsection{Selectivity of the nanocomposite detection platform}

To check the selectivity of our solid-phase platform, we tested the responses of the samples with interfering ions. These ions include $\mathrm{Ag}^{2+}, \mathrm{Al}^{3+}, \mathrm{Au}^{3+}, \mathrm{Ba}^{2+}, \mathrm{Ca}^{2+}$, $\mathrm{Cd}^{2+}, \mathrm{Co}^{2+}, \mathrm{Cr}^{3+}, \mathrm{Cu}^{2+}, \mathrm{Fe}^{2+}, \mathrm{Fe}^{3+}, \mathrm{Hg}^{2+}, \mathrm{K}^{+}, \mathrm{Mg}^{2+}, \mathrm{Mn}^{2+}, \mathrm{Na}^{+}, \mathrm{Pd}^{2+}, \mathrm{Pt}^{2+}$, and $\mathrm{Zn}^{2+}$ which were introduced at a concentration of $100 \mu \mathrm{M}$ both in the presence and absence of $1 \mu \mathrm{M} \mathrm{Pb}^{2+}$. Our system was still able to detect $\mathrm{Pb}^{2+}$ with selectivity in the range of 300- to 1000-fold (Figure 5-13). We also found that the selectivity toward several ions (e.g., $\mathrm{Ca}^{2+}, \mathrm{K}^{+}, \mathrm{Mg}^{2+}, \mathrm{Mn}^{2+}$, and $\mathrm{Na}^{+}$) was evidently improved when compared with detection in solution-phase (Figure 5-14). It is interesting to note that for the case of $\mathrm{Hg}^{2+}$, its presence induced an increase in peak absorbance instead of a decrease. While the exact mechanism behind this is unclear, we suspect that it might be related to the affinity of the pseudo ether crown structure of ethylene glycol groups toward $\mathrm{Hg}^{2+}$ 
ions ${ }^{259,260}$, by which adjacent AuNPs may form clusters in the presence of $\mathrm{Hg}^{2+}$ leading to enhanced localized surface Plasmon resonance.

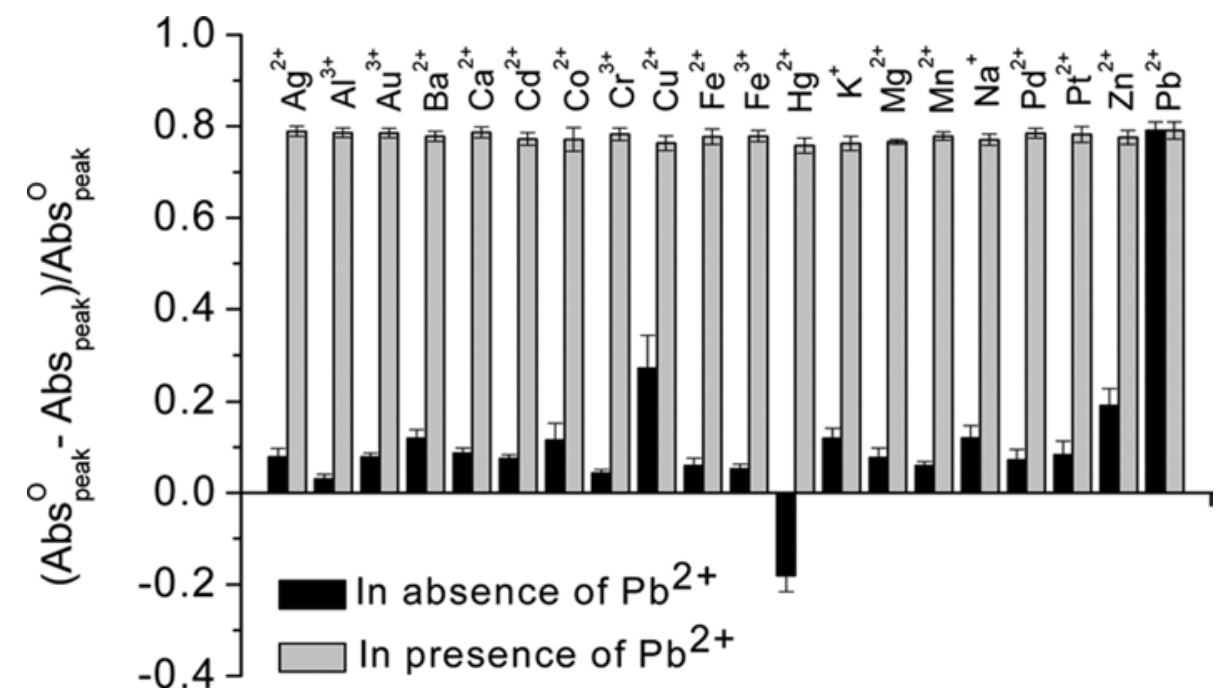

Figure 5-13. Responses to interfering ions at $100 \mu \mathrm{M}$ both in the absence and presence of $1 \mu \mathrm{M} \mathrm{Pb}^{2+}$. Error bars represent standard deviation $(n=3)$. Reprinted with permission from Anal. Chem., 2013, 85 (8), pp 4094-4099. Copyright 2013 American Chemical Society.

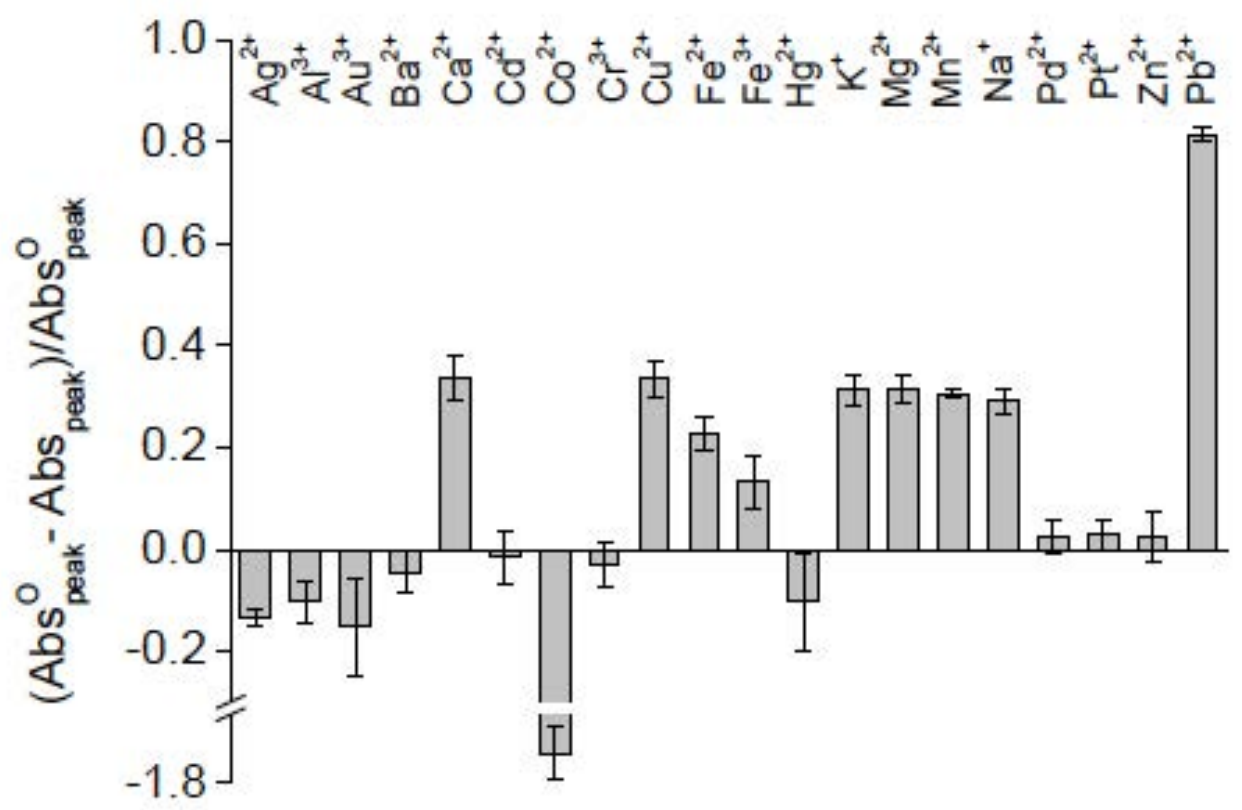

Figure 5-14. Responses to interfering ions at $100 \mu \mathrm{M}$ in the absence of $1 \mu \mathrm{M} \mathrm{Pb}^{2+}$. Error bars represent standard deviation $(n=3)$. Reprinted with permission from Anal. Chem., 2013, 85 (8), pp 4094-4099. Copyright 2013 American Chemical Society. 


\subsubsection{Lead ion detection from human serum}

Finally, lead detection in human serum was conducted using 1 h-POEGMA. Five levels of $\mathrm{Pb}^{2+}$ concentrations, $100 \mathrm{pM}, 500 \mathrm{pM}, 1 \mathrm{nM}, 10 \mathrm{nM}$, and $100 \mathrm{nM}$, were spiked to 5-fold diluted human serum. Five replicate analyses were performed for each sample to determine the recoveries defined as the difference between spiked sample result and unspiked sample result, divided by known spiked concentration. The results are listed in Table 5-2. We obtained averaged recoveries from the replicates, expressed in percentages, of $91.0-109.9 \%$ with percent relative standard deviations (which indicates how precise these average values are) of $1.76-2.10 \%$, showing the outstanding performance of our platform even in the presence of complex biological media. In comparison, the use of AuNPs on APTS-treated substrates resulted in significantly poorer recoveries and precision, which can be attributed to the instantaneous nonspecific adsorption of serum proteins on the AuNPs which in turn reduces the leaching efficiency. As we have shown for 1 h-POEGMA that AuNPs are embedded in the brush polymer, POEGMA acts as a selective barrier which denies nonspecific protein access, while still allowing $2 \mathrm{ME}$ and $\mathrm{Pb}^{2+}$ ions to access the AuNPs for detection to proceed as per normal. This is confirmed by X-ray photoelectron spectroscopy (XPS) (Figure 5-15). 
Table 5-2. Recoveries for the detection of $\mathrm{Pb}^{2+}$ in human serum $(n=5)$.

\begin{tabular}{cccccc} 
& \multicolumn{2}{c}{ 1 h-POEGMA } & & \multicolumn{2}{c}{ APTS-treated } \\
\cline { 2 - 3 } \cline { 5 - 6 } spiked amount $(\mathrm{nM})$ & recovery (\%) & RSD (\%) & & recovery (\%) & RSD (\%) \\
0.1 & 109.9 & 1.76 & & 274.6 & 4.38 \\
0.5 & 96.4 & 2.07 & & 273.7 & 1.42 \\
1 & 99.3 & 2.09 & & 276.6 & 12.42 \\
10 & 95.2 & 1.96 & & 233.8 & 9.27 \\
100 & 91 & 2.1 & & 195.2 & 7.99
\end{tabular}
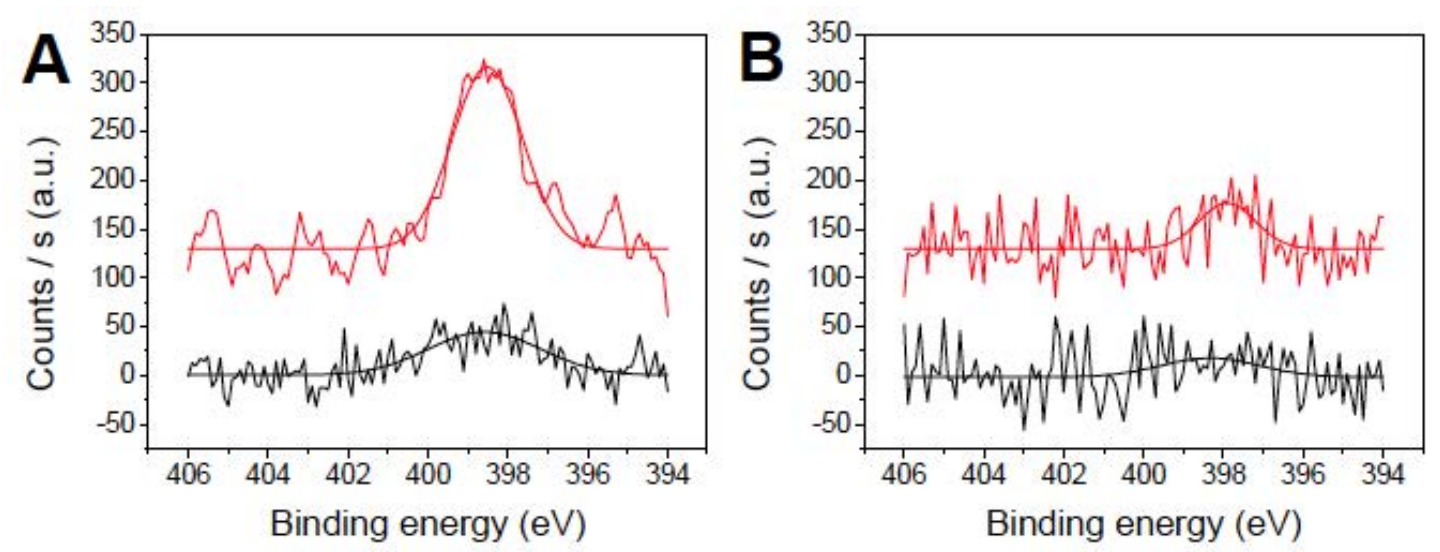

Figure 5-15. (A) N 1s XPS spectra obtained from APTS-AuNP substrates after detection of $1 \mu \mathrm{M}$ lead ions in serum (red trace) and water (black trace). (B) N 1s XPS spectra obtained from $1 \mathrm{~h}$-POEGMA-AuNP substrates after detection of $1 \mu \mathrm{M}$ lead ions in serum (red trace) and water (black trace). Compared to APTS-treated substrates, the N 1s spectra (used to imply the presence of amino acids on the surface) from 1 h-POEGMA substrates after lead detection from human serum revealed a significantly reduced peak implying the ability of POEGMA to deny non-specific adsorption of proteins. Reprinted with permission from Anal. Chem., 2013, 85 (8), pp 4094-4099. Copyright 2013 American Chemical Society.

Compared to other sophisticated methods developed recently ${ }^{255,261-275}$, our simple label-free technique presents among the lowest detection limit and broadest linear range for lead detection from water and complex biological fluid. A focused comparison of the performance of our platform against others which involve a solid 
interface $255,261-263,265,267-269,275$ is presented in Table 5-3. Our excellent recovery at concentrations as low as $100 \mathrm{pM}$ from human serum clearly demonstrates the excellent performance of our technique which can be used for real-world sample detection. Furthermore, as our polymer brush is grown on a glass surface, we can essentially transfer our platform from glass coverslips to optical fibers or glass capillary tubes. For example, the inner wall of a capillary tube can be coated with our polymer brush nanoparticle nanocomposite and can be placed in an optical fiber coupler ${ }^{276}$. With the advent of many commercially available miniaturized UV-vis fiber light source, such configuration can prospectively be packaged within a portable setup.

Table 5-3. A comparison with other recently developed methods for the detection of $\mathrm{Pb}^{2+}$ involving a liquid/solid interface.

\begin{tabular}{|l|c|c|c|c|}
\hline \multicolumn{1}{|c|}{ technique } & LOD & linear range & selectivity & ref. \\
\hline colorimetry & $0.025 \mathrm{nM}$ & $0.1-100 \mathrm{nM}$ & $300-1000$-fold & this work \\
\hline colorimetry & $0.050 \mathrm{nM}$ & $0.1-1.5 \mathrm{nM}$ & $\geq 100$-fold & 255 \\
\hline fluorometry & $10 \mathrm{nM}$ & $50-1000 \mathrm{nM}$ & $10-100$-fold & 262 \\
\hline mass spectrometry & $0.5 \mathrm{nM}$ & $1 \mathrm{nM}-5 \mu \mathrm{M}$ & 100 -fold & 265 \\
\hline electrochemical, voltammetry & $\begin{array}{c}0.546 \mathrm{ppb} \\
(2.635 \mathrm{nM})\end{array}$ & $3.31-22.29 \mathrm{ppb}$ & N/A & 267 \\
\hline electrochemical, voltammetry & $0.32 \mathrm{nM}$ & $0.59 \mathrm{nM}-10 \mathrm{mM}$ & N/A & 263 \\
\hline electrochemical, voltammetry & $0.14 \mathrm{nM}$ & $10 \mathrm{nM}-1.5 \mu \mathrm{M}$ & N/A & 261 \\
\hline electrochemical, voltammetry & $1 \mathrm{nM}$ & $5 \mathrm{nM}-0.8 \mu \mathrm{M}$ & $50-200$-fold & 268 \\
\hline electrochemical, voltammetry & $0.5 \mathrm{ppb}$ & $0-50 \mathrm{ppb}$ & N/A & 275 \\
\hline electrochemical, potentiometry & $870 \mathrm{nM}$ & $4.3 \mu \mathrm{nM}-10 \mathrm{mM}$ & N/A & 269 \\
\hline
\end{tabular}

\subsection{Conclusion}

In conclusion, we have shown a simple fabrication method for a solid-phase colorimetric sensor by loading unmodified AuNPs into POEGMA matrix grown on glass substrates via simple immersion. The loading of AuNPs into a POEGMA matrix was achieved without any chemical modification. We then studied the effect of brush 
thickness on AuNP loading and the subsequent detection performance for lead detection. This was demonstrated through the successful transference of detection chemistry for lead sensing from the solution phase to the solid phase. We obtained lowest LOD using 1 h-POEGMA and widest dynamic range using 3 h-POEGMA. We have shown that our solid-phase detection platform can detect lead ions with 300 - to 1000-fold selectivity. Finally, we have demonstrated that detection of lead ions from human serum can be performed by simple dilution without compromising the detection performance. 


\section{Chapter 6}

\section{Three-Dimensional Gold Nanoparticle Incorporation in POEGMA via In-Stacking and Adsorption on Dual Round Growth of POEGMA for Plasmonic Sensing of Thrombin}

\subsection{Introduction}

Nanoparticle polymer composites represent a class of highly prospective materials $^{105,106}$. Recent reports employing nanoparticles in end-tethered polymer brushes have highlighted feasible roles in the exciting fields of photovoltaics ${ }^{192}$, magnetically-driven nanoactuation ${ }^{190}$ and $\mathrm{pH}^{193}$, temperature ${ }^{277}$ and molecular sensing ${ }^{191}$.

Several groups have attempted nanoparticle incorporation by subjecting the polymer brushes and/or nanoparticles to post-modification procedures, including polymer encapsulation ofnanoparticles ${ }^{189,191,235,278}$. For end-tethered polymer brushes, it has been proposed that, at a given polymer graft density, nanoparticles can either fully infiltrate into or remain on the upper portion of the brush depending on their size 235,237 . Although the use of brushes, such as poly(N-isopropyl acrylamide) $(\text { PNIPAAm) })^{277}$ and poly(4-vinylpyridine-b-styrene) (P4VP-b-PS) ${ }^{188}$ with respective grafting densities of 0.15 and 0.20 chains $\mathrm{nm}^{-2}$, have immobilized gold nanoparticles (AuNPs) of sizes $\sim 5$ and $\sim 2 \mathrm{~nm}$, respectively, true infiltration was well-documented only in the case of the latter, where the nanoparticle size is close to the inter-grafting distance $(\sim 2.2 \mathrm{~nm})$. Hence, a persistent challenge exists in achieving three-dimensional (3D) assembly of nanoparticles in areas where the use of brushes with even higher grafting densities is crucial, such as to render protein or bacterial resistance. 
In this contribution, we report a facile approach to achieve tunable loading of asprepared AuNPs in unmodified poly(oligo(ethylene glycol) methacrylate) (POEGMA) brushes which have been proven to possess supreme non-fouling properties ${ }^{225}$. To the best of our knowledge, there is no prior report on AuNP loading into macrophase POEGMA. This may be due to the branched nature of POEGMA yielding a bottlebrush morphology. In addition, POEGMA brushes are grown at relatively high grafting densities. These factors typically hamper nanoparticleinfiltration ${ }^{237}$. Furthermore, any attempts to chemically modify the brush might jeopardize its nonfouling properties.

It has been suggested that polyethylene glycol grafts can displace citrate molecules which are used to stabilize colloidal AuNPs, ultimately resulting in a multidentate interaction with a gold surface ${ }^{253}$. This interaction originates from ethylene glycol (EG) groups which form a pseudocrown ether structure capable of binding to gold with high affinity. Since the side branches of POEGMA are abundant with EG groups, AuNPs which cannot penetrate can initially adsorb on top of the brush, forming a monolayer. However, such a configuration is effectively twodimensional (2D) and does not fully exploit the 3D spatial matrix of the brush. To overcome the barrier associated with high graft density and achieve maximized, 3D nanoparticle loading, we introduce an 'in-stacking' strategy which allows nanoparticles to be incorporated within the polymer brush matrix via a series of immersion steps, ultimately resulting in a high density loading of AuNPs. 


\subsection{Specific Methods}

\subsubsection{Gold nanoparticle incorporation via in-stacking}

Substrates were immersed in a fresh $3 \mathrm{~mL}$ solution of AuNP of specified diameters for a stipulated duration. Upon removal, they were copiously rinsed with deionized $\mathrm{H}_{2} \mathrm{O}$. They were then rinsed 3 times in deionized $\mathrm{H}_{2} \mathrm{O}$ under orbital shaking at $120 \mathrm{rpm}$ for $2 \mathrm{~min}$ each and finally blown dry with $\mathrm{N}_{2}$. For repeat immersions, the same AuNP solution was used.

\subsubsection{Two rounds growth of POEGMA}

The POEGMA was grown from glass coverslips following the main protocol presented in Chapter 3. For 2nd round POEGMA growth, polymerization was repeated on the same substrates; however, with varying volumetric ratio of methanol and MilliQ water.

\subsubsection{Gold nanoparticle incorporation of POEGMA grown for two rounds}

Substrates were immersed in a fresh $3 \mathrm{~mL}$ solution of AuNP, $18 \mathrm{~nm}$ in diameter, overnight. The substrates were then thoroughly rinsed 3 times in deionized $\mathrm{H}_{2} \mathrm{O}$ under orbital shaking at $120 \mathrm{rpm}$ for 2 min each and finally blown dry with $\mathrm{N}_{2}$.

\subsubsection{Gold nanoparticle postfunctionalization and thrombin detection}

As previously reported ${ }^{111,279}$, thiol groups in the aptamer were first activated before chemical modification of the AuNPs with thrombin-binding aptamer (TBA) was conducted. In brief, $0.1 \mathrm{mM}$ tris(2-carboxyethyl)phosphine (TCEP) in $50 \mathrm{mM}$ acetate buffer ( $\mathrm{pH}$ 5.2) was used to deprotect $0.1 \mathrm{mM} 5$ '-thiol aptamer for $1 \mathrm{~h}$ at room temperature. Deprotected aptamer was then diluted to $0.1 \mu \mathrm{M}$ with Milli-Q water and substrates were immersed in the solution for another $1 \mathrm{~h}$. The substrates were then 
thoroughly rinsed under Milli-Q water and blow dried under nitrogen. For thrombin detection, thrombin stock solutions were diluted with thrombin binding buffer to the desired concentrations. The aptamer-functionalized substrates were then immersed in the solution for 1 hour before rinsing thoroughly with Milli-Q water and dried under nitrogen stream.

\subsubsection{Characterization}

Thickness measurements were obtained from the silicon substrates by ellipsometry in air using a J.A. Woolam $\alpha$-SE spectroscopic ellipsometer. UV-vis spectra were obtained using a Shimadzu UV-2540 UV spectrophotometer. Atomic Force Microscopy data were obtained in air from an Asylum Research MFP-3D AFM system conducted in tapping mode. Scanning electron micrographs were obtained from JEOL JSM-6700F FESEM microscope. Except for AuNPs on APTS, all samples were coated with $\mathrm{Pt}$ for $30 \mathrm{sec}$ at $20 \mathrm{~mA}$.

\subsection{Results and Discussion}

\subsubsection{In-stacking of nanoparticle in brush of different thickneeses}

The proposed mechanism for in-stacking is summarized in Figure 6-1A. Briefly, at initial immersion, AuNPs are adsorbed on the top region of the brush with its 2D coverage increasing with time. When the sample is dried, the brush collapses. Upon re-immersion, polymer chains at regions free from the adsorbed AuNPs would reswell with a higher degree of conformational freedom. This facilitates successive capture of AuNPs which would be stacked into the brush, as it collapses again when dried. This process can continue until the number of chains, which can freely re-swell upon re-immersion, is insufficient to capture an additional layer of AuNPs. 


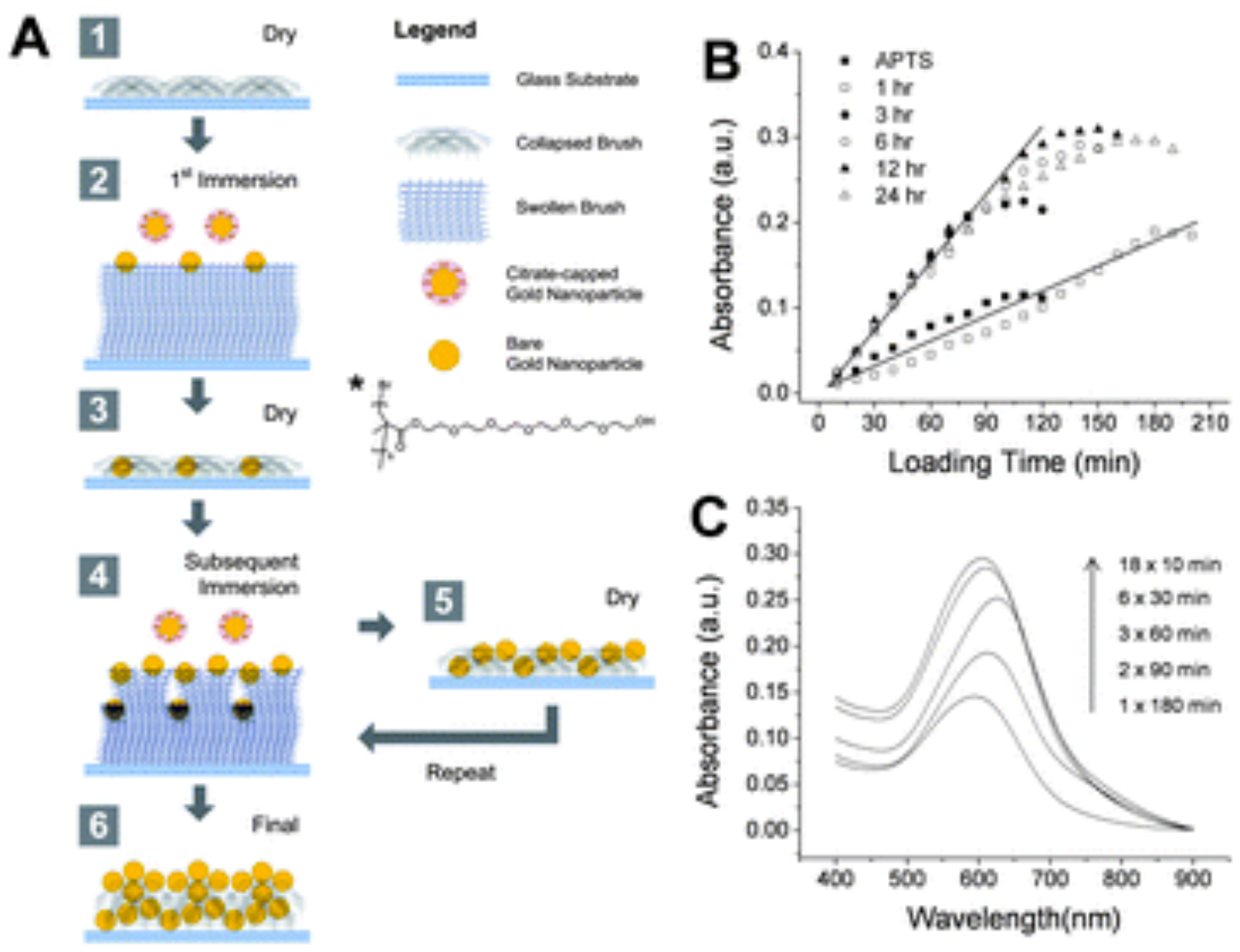

Figure 6-1. (A) Schematic of the in-stacking process (1-6) showing the brush when dry (1), immersed in AuNP solution (2), washed and dried (3), re-immersed in AuNP solution (4), washed and dried (5), and the final state when subjected to repeated rounds of immersions (6). Asterisk denotes the side branch structure of POEGMA; the number of EG repeats may vary slightly. B) Variation of peak absorbance with loading time of AuNP in polymer brushes with different synthesis durations ( $1 \mathrm{~h}$ to $24 \mathrm{~h})$. (C) Final UV-vis spectra of polymer brush coated glass coverslips after being subjected to different AuNP loading patterns. (J. Mater. Chem., 2012,22, 1274-1277 - Reproduced by permission of The Royal Society of Chemistry)

To prove the feasibility of in-stacking, we investigated the AuNP loading density versus immersion time. POEGMA was grown on glass coverslips via surface-initiated atomic transferradical polymerization (SI-ATRP) for synthesis durations ranging from 1 to $24 \mathrm{~h}$. Ellipsometry revealed dry thicknesses varying from $\sim 20 \mathrm{~nm}$ to $\sim 100 \mathrm{~nm}$ (Figure 6-2). By employing mathematical correlations proposed in recent works ${ }^{237,280,}$ 
${ }^{281}$, we estimate respective graft densities of $\sim 0.35$ to $\sim 1.89$ chains $\mathrm{nm}^{-2}$ depending on the synthesis time. This translates to local surface polymer coil sizes or 'surface blob sizes' $\left(\xi_{\text {surf }}\right.$ ) of $\sim 5.22$ to $\sim 2.98 \mathrm{~nm}$ (sample calculations are presented in APPENDIX). We proceeded to immerse the coverslips in $13 \mathrm{~nm}$ AuNP solution for as short as 10 $\min (13 \mathrm{~nm}$ was chosen as it is the typical size of AuNPs obtained from the commonly used citrate reduction Turkevich method; the in-stacking with various sizes of AuNP is presented later in this communication). The AuNPs would merely adsorb on the surface, as they are devoid of brush penetration $\left(13 \mathrm{~nm}>\xi_{\text {surf }}\right)^{237}$. Upon repeated immersion of the coverslips in the AuNP solution, UV-vis spectra from dry coverslips revealed a steady increase in absorbance intensity with gradual red-shifting of the peak position (Figure 6-3), indicating increased nanoparticle packing ${ }^{257,282}$.

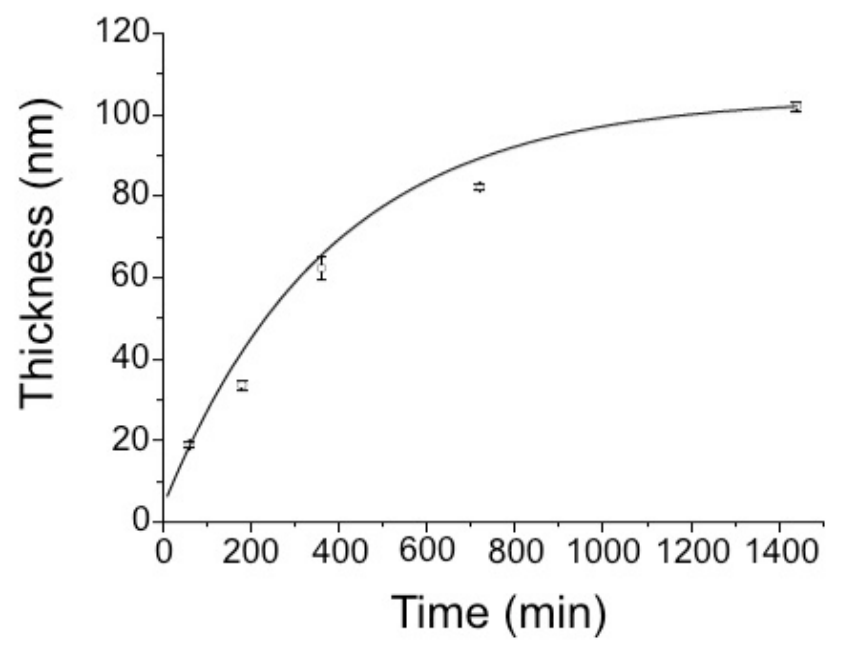

Figure 6-2. Ellipsometric thickness of polymer brush as a function of time. (J. Mater. Chem., 2012,22, 1274-1277 - Reproduced by permission of The Royal Society of Chemistry) 


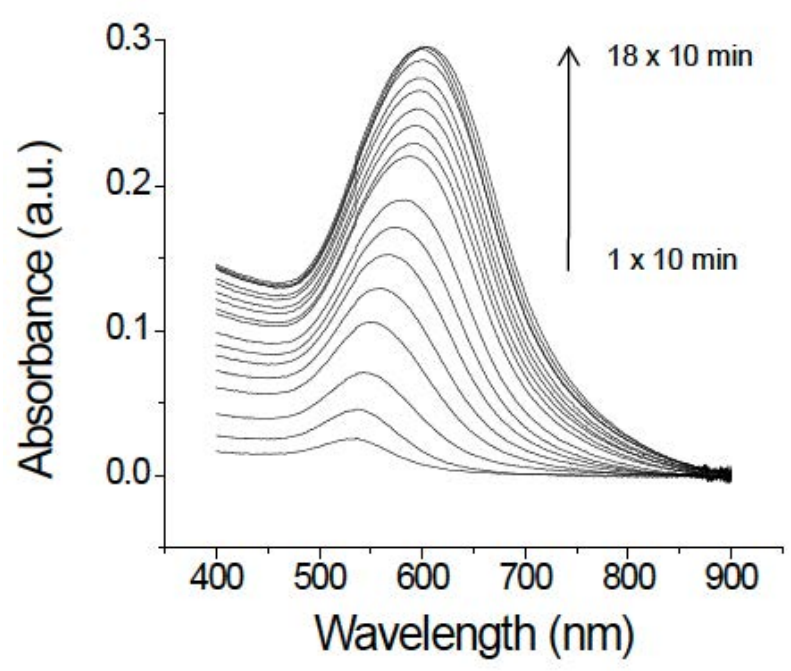

Figure 6-3. UV-vis spectra of 24-hr POEGMA coated glass coverslips after each 10 min cycle for $13 \mathrm{~nm}$ AuNPs. (J. Mater. Chem., 2012,22, 1274-1277 - Reproduced by permission of The Royal Society of Chemistry)

The plot of peak UV-vis absorbance versus cumulative $13 \mathrm{~nm}$ AuNP loading time is presented in Figure 6-1B. The initial gradient of the increase in absorbance for $3 \mathrm{~h}$ POEGMA and above is approximately 1.5 times steeper than that of $1 \mathrm{~h}$ POEGMA. The latter shares roughly the same gradient to the APTS-treated substrate. We can therefore infer that, for 3, 6, 12 and 24 h POEGMA, the AuNP loading extends beyond the 2D profile, while the depth of $1 \mathrm{~h}$ POEGMA does not accommodate in-stacking. Between aminopropyltriethoxysilane (APTS)-modified substrate and POEGMA synthesized for $1 \mathrm{~h}(1 \mathrm{~h}$ pOEGMA), a higher maximum loading is attainable with more cycles in the case of the latter. This suggests that the $1 \mathrm{~h}$ POEGMA provides more binding sites for monolayer formation relative to the APTS. Such higher AuNP loading on the $1 \mathrm{~h}$ POEGMA could be attributed to the role of EG side chains. Firstly, abundant EG groups per grafting site lead to more effective binding for the AuNPs in comparison to APTS, on which only a single amino group is present. Secondly, when EG displaces citrate molecules, the immobilized AuNPs approach charge neutrality, 
resulting in the reduction of repulsive effects between immobilized and approaching AuNPs.

\subsubsection{Effects of different times per cycle}

To investigate our speculation on the AuNP assembly profile, we proceeded to load AuNPs into $24 \mathrm{~h}$ POEGMA with the following five different immersion times per cycle $(10,30,60,90$ and $180 \mathrm{~min})$ for the same total loading duration of $3 \mathrm{~h}$. A decreased loading was observed with longer cycle immersion times (Figure 6-1C). Compared to 18 immersion cycles of 10 min each, a single $3 \mathrm{~h}$ immersion yielded less than half AuNP loading. This is in agreement with our proposed stacking mechanism; as AuNPs tend to approach a saturated monolayer with increasing immersion time, less of the polymer brush can freely re-swell to capture new AuNPs during subsequent immersions for in-stacking.

The existence of a 3D assembly was also inferred by comparing UV-vis spectra of blown dry and wet coverslips at every $30 \mathrm{~min}$ interval of six immersion cycles. Spectral blue shifts of varying degrees were observed when switching from dry to wet states (Figure 6-4). The blue shift can be attributed to the increased separation distance between AuNPs arising from swelling of the polymer brush ${ }^{277}$. After the first immersion, no peak shift occurred between dry and wet states. This is because AuNPs in the monolayer rises with the same vertical displacement when the brush is swollen. After the second immersion, a significant blue shift of $\sim 14 \mathrm{~nm}$ was observed due to the different degrees of swelling between chains attached to the first and second layer AuNPs. Blue shifting becomes less apparent after subsequent cycles, reaching a minimal shift of $\sim 2 \mathrm{~nm}$ after the sixth cycle. The difference in degree of swelling is gradually suppressed with increased AuNP loading due to spatial constraints and increased chain perturbations. The observation that AuNPs rise with the swelling 
brush indicates that they are firmly fixed to the POEGMA chains once citrate molecules are displaced by the pseudocrown ether structures of EG groups in the side chains.

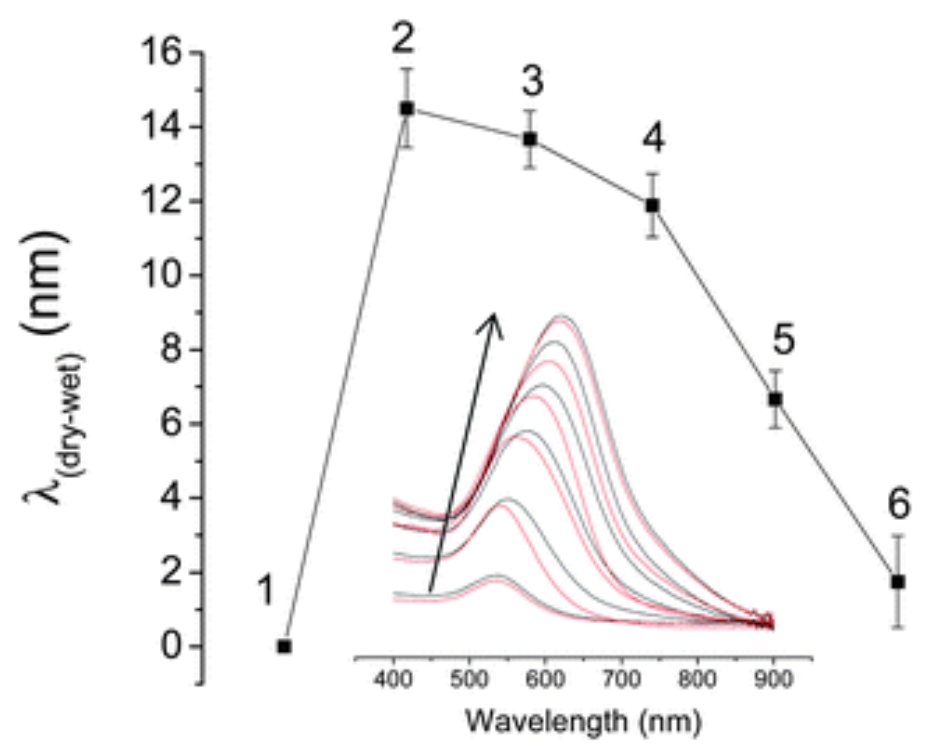

Figure 6-4. Peak shift between dry and wet states for polymer brush coated glass coverslips after each cycle of 30 min immersions plotted against the number of cycles. Inset depicts corresponding UV-vis spectra showing dry (black line) and wet (red line) states. Arrow indicates increasing number of cycles. (J. Mater. Chem., 2012,22, 12741277 - Reproduced by permission of The Royal Society of Chemistry)

\subsubsection{Mechanism behind in-stacking}

To gain deeper insight into the proposed in-stacking mechanism at different stages, we compared atomic force microscopy (AFM) height and phase retraces as well as corresponding UV-visspectra obtained at dry conditions from $3 \mathrm{~h}$ POEGMA and $24 \mathrm{~h}$ POEGMA subjected to immersion cycles of $30 \mathrm{~min}$ each (Figure 6-5). Note that $1 \mathrm{~h}$ POEGMA is omitted in this comparison since AuNP assembly followed a 2D profile. After initial loading, the surface morphology of 3 h POEGMA reveals several distinct bumps arising from AuNPs amidst the collapsed POEGMA (Figure 6-5A). In contrast, 24 h POEGMA shows a relatively even surface similar to pristine POEGMA, 
indicating fully embedded AuNPs in the POEGMA matrix due to the long polymer chains (Figure 6-5B). Root mean square (RMS) roughness values from their height retraces are $0.72 \mathrm{~nm}$ and $0.56 \mathrm{~nm}$, respectively. However, their phase retraces indicate similar 2D coverage of AuNPs and UV-vis spectra infer similar total loading density.

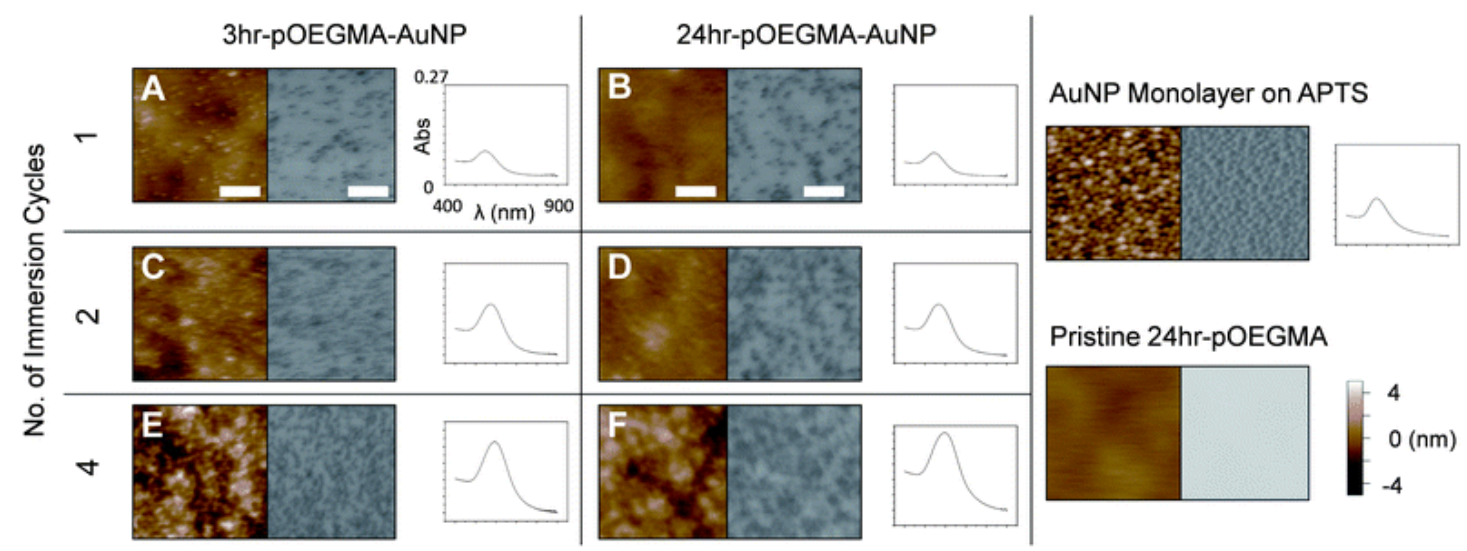

Figure 6-5. Sets of AFM height retrace, phase retrace and corresponding UV-vis spectra for (A) $3 \mathrm{~h}$ POEGMA and (B) $24 \mathrm{~h}$ POEGMA after a single cycle of $30 \mathrm{~min}$ AuNP loading, (C) $3 \mathrm{~h}$ POEGMA and (D) $24 \mathrm{~h}$ POEGMA after 2 cycles, (E) $3 \mathrm{~h}$ POEGMA and (F) 24 h POEGMA after 4 cycles. Sets for AuNP monolayer on APTS and pristine $24 \mathrm{~h}$ POEGMA are included for comparison. AFM scale bar represents 200 nm. (J. Mater. Chem., 2012,22, 1274-1277 - Reproduced by permission of The Royal Society of Chemistry)

After the second immersion, there is only a slight difference in surface morphology for both 3 and 24 h POEGMA (Figure 6-5C \& D). Their RMS roughness increased slightly to $0.81 \mathrm{~nm}$ and $0.59 \mathrm{~nm}$, respectively. While their phase retraces also show similar 2D surface coverage, their corresponding UV-vis spectra reflect an almost double increase in absorbance, indicating a higher amount of total AuNP loading. Through this evidence, we can conclude that there is an underlying layer of AuNPs as a result of stacking. 
Obvious changes in surface morphology occur after four rounds of immersion for both 3 and 24 h POEGMA (Figure 6-5E \& F). Their RMS roughness increased to $1.20 \mathrm{~nm}$ and $0.99 \mathrm{~nm}$, respectively. While maximal loading has almost been reached for the case of $3 \mathrm{~h}$ POEGMA, it is not so for $24 \mathrm{~h}$ POEGMA; this is why the surface roughness of $3 \mathrm{~h}$ POEGMA $(1.20 \mathrm{~nm})$ is close to that of an AuNP monolayer on APTS $(1.32 \mathrm{~nm})$ while the surface of $24 \mathrm{~h}$ POEGMA remains comparatively smooth albeit with a slightly higher UV-vis absorbance.

Field emission scanning electron micrographs (FESEM) show the morphology of dried POEGMA-AuNP sample at an anticipated close-to-maximal loading for $3 \mathrm{~h}$ POEGMA obtained via 4 immersion cycles of 30 min each. From the top view, AuNPs in $3 \mathrm{~h}$ POEGMA are highly close-packed and are encapsulated within the polymer brush matrix (Figure 6-6A). Even so, we confirm that they are not aggregated because the UV-vis spectrum still exhibits a distinct peak (Figure 6-1C). In comparison, AuNPs on APTS mostly exist as singular entities which are more spaced apart (Figure 6-6B). An image of the sample edge obtained at a $45^{\circ}$ tilt angle confirms the 3D configuration of in-stacked AuNPs which are vertically dense (Figure 6-6C). The thickness of $3 \mathrm{~h}$ POEGMA is estimated to be approximately 50 $\mathrm{nm}$ which is larger than the pristine brush thickness of $\sim 30 \mathrm{~nm}$, indicating that some of the free chains indeed swell more than $30 \mathrm{~nm}$ to capture the AuNPs. 


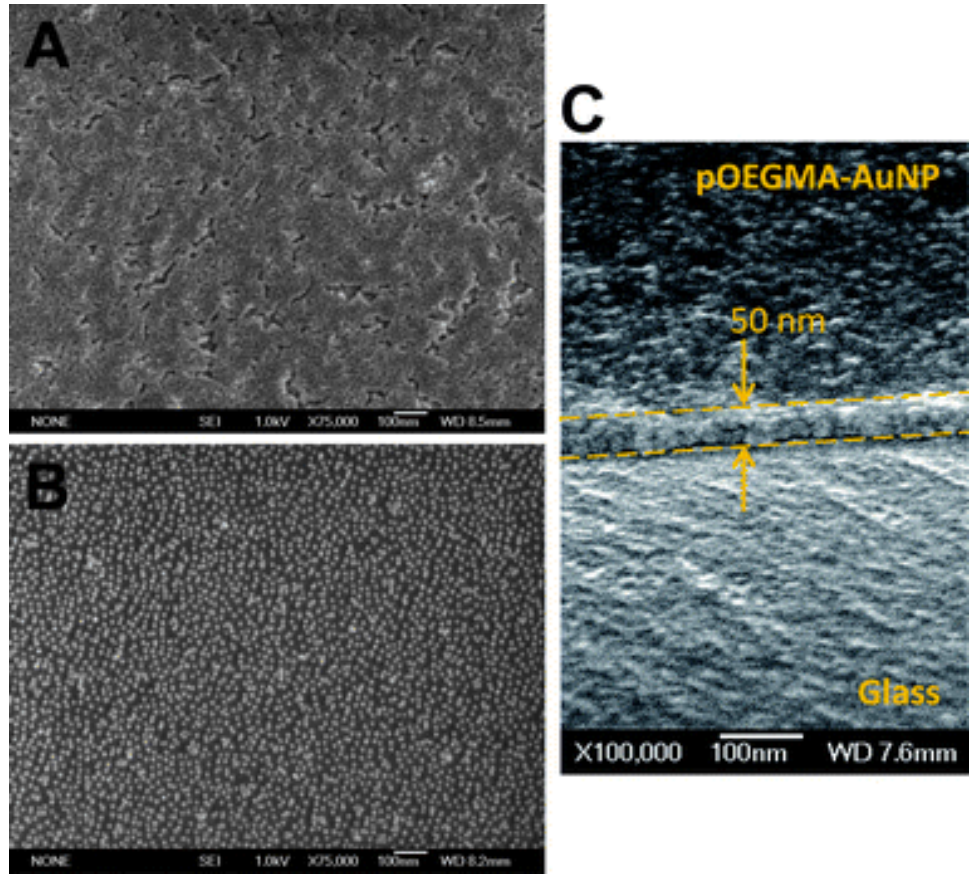

Figure 6-6. FESEM images of (A) AuNPs loaded into $3 \mathrm{~h}$ pOEGMA, (B) single monolayer of AuNP on APTS-treated surface, and (C) edge of $3 \mathrm{~h}$ pOEGMA-AuNP composite on glass obtained at a $45^{\circ}$ tilt angle showing the configuration of in-stacked AuNPs (the sample is Pt-coated at a tilted angle for $30 \mathrm{~s}$ at $20 \mathrm{~mA}$ ). (J. Mater. Chem., 2012,22, 1274-1277 - Reproduced by permission of The Royal Society of Chemistry)

\subsubsection{Feasibility of in-stacking nanoparticles of different sizes}

To investigate the validity of the proposed mechanism for other sizes, the nanoparticle stacking in polymer brushes using 5, 23 and $31 \mathrm{~nm}$ AuNPs was explored. During repeated immersions, there was a unique observation for $5 \mathrm{~nm}$ AuNPs. After the third cycle, a drop in absorbance accompanied by a significant red-shift occurred (Figure 67A), indicating cluster formation within the POEGMA matrix. On the other hand, the larger nanoparticles shared a similar trend with $13 \mathrm{~nm}$ AuNPs (Figures 6-7B \& C). By comparing the maximum absorbance attainable after repeated immersions (black trace) and after a single overnight immersion (red trace) in Figures 6-7A-C, we conclude that in-stacking is most effective in a size window of around $\sim 10-30 \mathrm{~nm}$. 
Below this range, particles which are sufficiently small would undergo simple infiltration, as in the case of $5 \mathrm{~nm}$ AuNPs which were able to permeate the brush and form clusters. Above this range, particles would be too large to be accommodated by the depth of the brush so that they simply adsorb on the surface. This is supported by the respective FESEM images which show different morphologies of dried POEGMAAuNP sample with AuNPs of different sizes (Figures 6-7D-F). The presence of distinct ridges in Figure 6-7E as well as Figure 6-6A, for $23 \mathrm{~nm}$ and $13 \mathrm{~nm}$ AuNPs respectively, indicates that as the brush accommodates a large amount of AuNPs during in-stacking, they are dragged inwards during brush collapse, resulting in separation fronts. Such ridges are not as distinct for the cases of $5 \mathrm{~nm}$ and $31 \mathrm{~nm}$ AuNPs (Figures 6-7D \& F). 

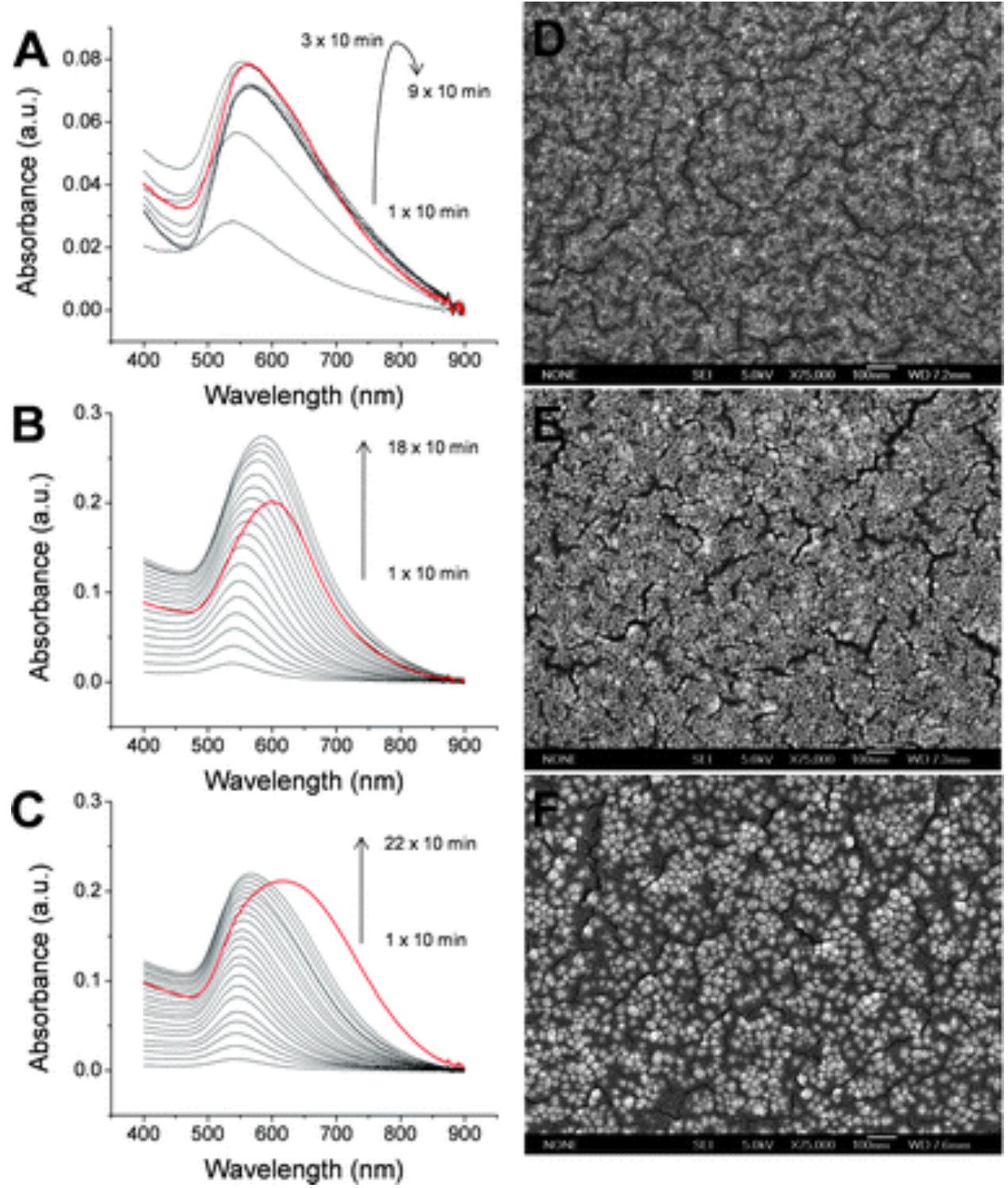

Figure 6-7. UV-vis spectra of $24 \mathrm{~h}$ pOEGMA coated glass coverslips after each 10 min immersion cycle up to maximum absorbance as well as after single overnight immersion (red trace) for 5, 23 and $31 \mathrm{~nm}$ AuNPs $(\mathrm{A}-\mathrm{C})$. Corresponding FESEM images obtained from $3 \mathrm{~h}$ pOEGMA after maximum number of cycles for 5, 23 and 31 nm AuNPs (D-F); $3 \mathrm{~h}$ pOEGMA was used for FESEM for clearer visualization. ( $J$. Mater. Chem., 2012,22, 1274-1277 - Reproduced by permission of The Royal Society of Chemistry) 


\subsubsection{Rough surface POEGMA based on dual rounds of brush synthesis}

In relation to our finding that AuNPs could be in-stacked, or deeply embedded within the polymer brush, we proceeded to study the effects of having a rougher surface on the immobilization of AuNPs. In order to achieve this, we synthesized two rounds of POEGMA with the 1st round POEGMA grown under a fixed volummetric water/methanol solvent ratio $(20: 80 \mathrm{v} / \mathrm{v})$ for $3 \mathrm{~h}$ while the 2 nd round grown under a fixed volummetric water/methanol solvent ratio (80:20 v/v) POEGMA for $6 \mathrm{~h}$. Due to the difference in grafting densities, we found that completely reversing the solvent ratio of the $1 \mathrm{st}$ and 2 nd rounds (i.e. from water/methanol $20: 80 \mathrm{v} / \mathrm{v}$ to $80: 20 \mathrm{v} / \mathrm{v}$ resulted in the roughest surface morphology. This could be seen from the AFM results, where the surface morphology of the 2 rounds is evidently higher than 1 round. RMS surface roughness increases from $0.898 \mathrm{~nm}$ to $1.466 \mathrm{~nm}$ and larger aggregate domains could also be observed for the 2 rounds. This is clearly seen from the AFM 3D profiles, whereby a rougher surface morphology is attained for 2 rounds in comparison to 1 round (Figure 6-8).

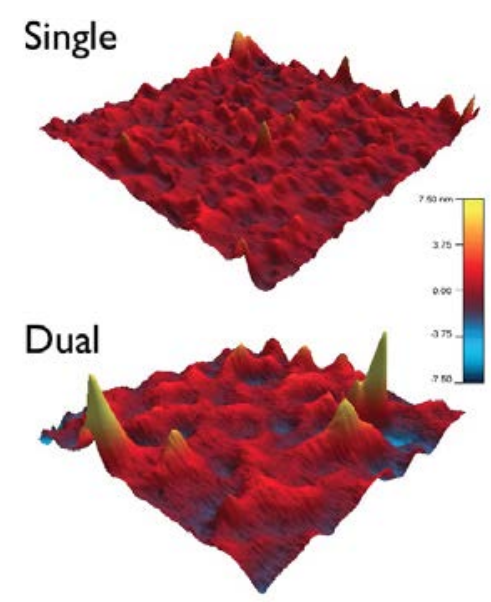

Figure 6-8. AFM 3-dimensional height profiles of pristine POEGMA with single (top) and dual (bottom) rounds of POEGMA synthesis. 


\subsubsection{Nanoparticle incorporation on rough surface POEGMA}

To investigate the AuNP loading capacities of polymer brushes with different roughness, substrates functionalized with 1 and 2 rounds of POEGMA brushes $\left(2^{\text {nd }}\right.$ POEGMA growth reaction time $=6 \mathrm{~h}$ ) were immersed in $18 \mathrm{~nm}$ AuNPs overnight and rinsed thoroughly before characterization. The cross-sectional FESEM images and AFM data for both 1 and 2 rounds POEGMA loaded with $18 \mathrm{~nm}$ AuNPs overnight are obtained (Figure 6-9), and the surface roughness are analyzed using AFM in tapping mode. Instead of giving a rougher surface (similar to the before loading), the 2 rounds POEGMA brush exhibits a smoother surface with RMS surface roughness of 1.477 $\mathrm{nm}$ in comparison to the 1 round POEGMA with RMS surface roughness of $1.690 \mathrm{~nm}$. Also, the AuNPs on 1 round POEGMA appears to be sitting on the surface unlike the 2 rounds POEGMA. We believe that the loosely held end chains will wrap around the particles, making it possible for the brush to be smoother under AFM.
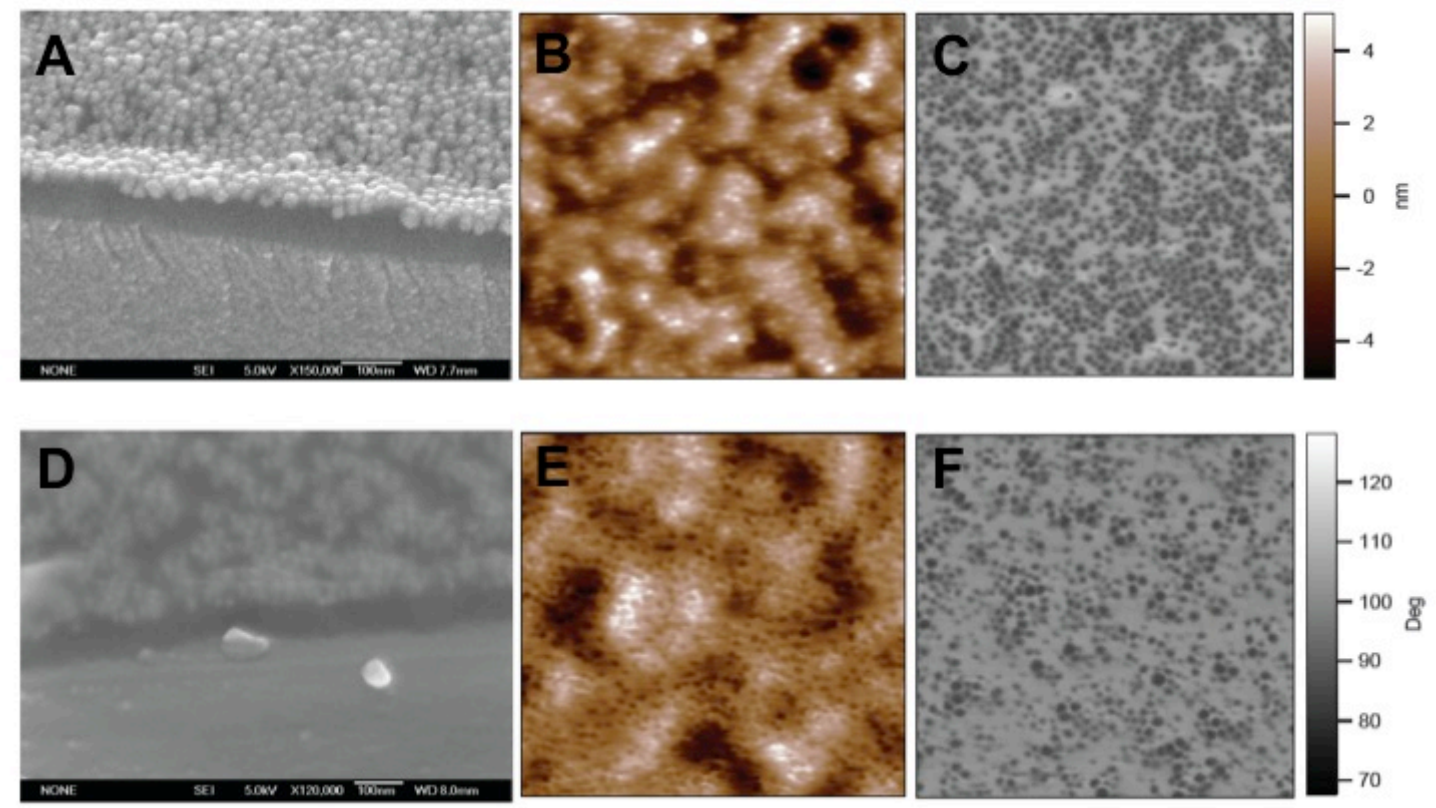

Figure 6-9. Cross-sectional FESEM micrographs, AFM height and phase retraces of POEGMA-AuNP nanocomposites with single (A-C) and dual (D-F) rounds of POEGMA synthesis. 


\subsubsection{Thrombin sensing based on mobility of nanoparticles on rough POEGMA}

To demonstrate the applicability of the AuNP-POEGMA nanocomposite as a solid-phase biosensing platform, we employ a sensing mechanism analogous to those performed in the solution phase in detecting a coagulation protein, human $\alpha$-thrombin based on aptamer binding. Thrombin-binding aptamer (TBA), a nucleic acid, binds to thrombin with high affinity due to its capability to form a quadraplex structure ${ }^{111,283}$, ${ }^{284}$. Furthermore, aptamers can be easily thiolated ${ }^{285-287}$ to bind to gold via chemical (thiol-gold interaction) and physical adsorption (through nitrogenous bases) $)^{285}$.

There has been an extensive amount of work in literature which reports on the conventional colorimetric detection of thrombin (i.e. solution phase), involving aggregation of AuNPs upon addition of human $\alpha$-thrombin ${ }^{113,288}$. This is attributed by attachment of TBA immobilized on the AuNPs surface with the thrombin molecules, resulting in aggregation of the nanoparticles and thus, a red-shift in the absorbance peak. However, at present, colorimetric detection of thrombin in the solid phase is limited; none of which has reported the use of colorimetric detection mechanisms in the solid phase. To address the current limitations of detection schemes in the solution phase (i.e. limited to testing of clear samples and poor stability of the nanoparticles), we propose a novel sensing mechanism which relies upon aggregation/de-aggregation of nanoparticles on a solid substrate by using TBA modified with a thiol functional group at the $5^{\prime}$ end for covalent binding to the gold surface. We hypothesized that AuNPs in 2 rounds POEGMA brush will demonstrate a different binding capability to TBA and subsequently, thrombin molecules due to the nanomobility of the nanoparticles in the brush (Figure 6-10). 

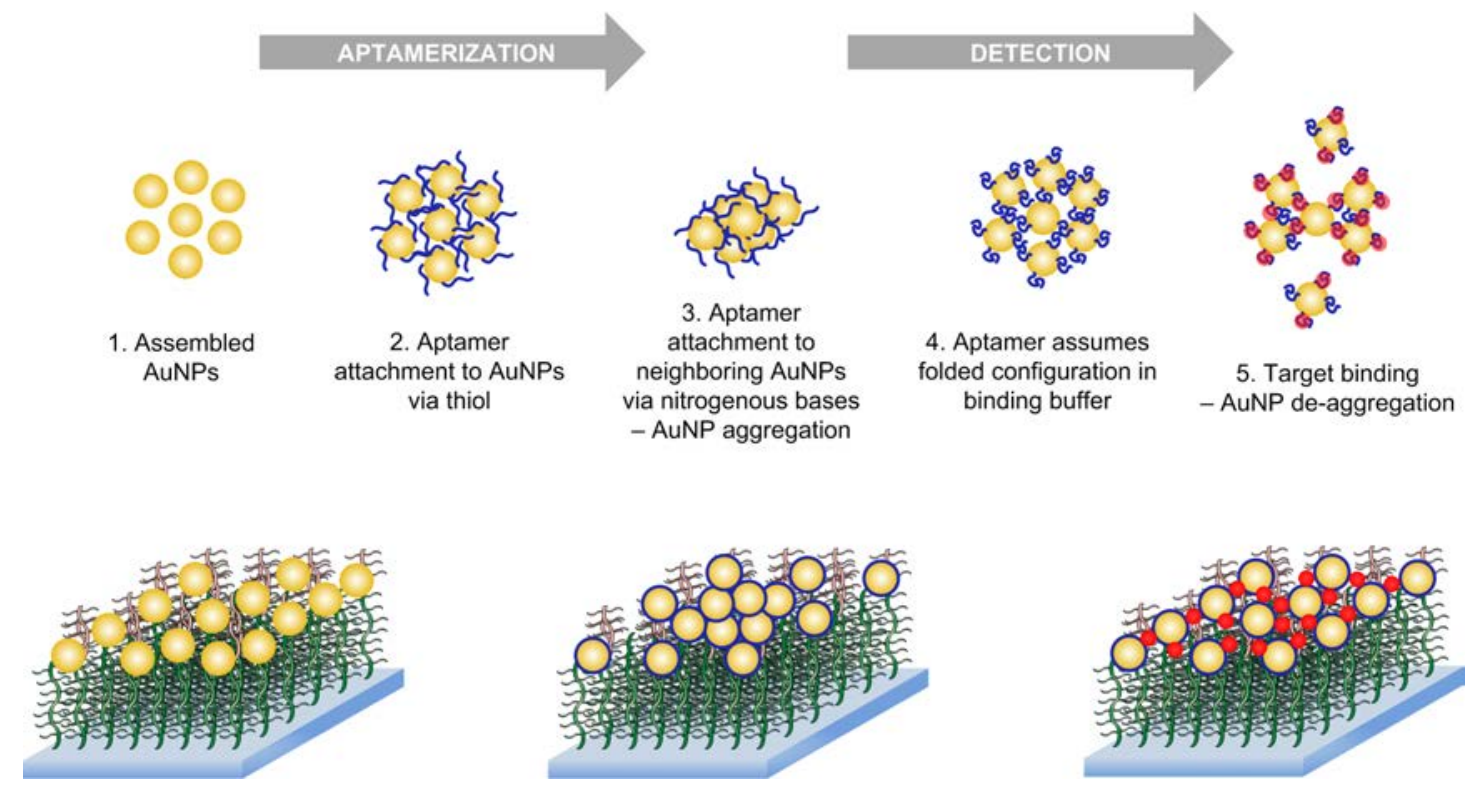

Figure 6-10. Simplified schematic illustrating AuNP aptamerization with thrombinbinding aptamer followed by thrombin detection.

To prove this hypothesis, $18 \mathrm{~nm}$ AuNPs immobilized in 2 rounds POEGMA brush are functionalized with $0.1 \mu \mathrm{M}$ TBA for 1 hour, followed by the introduction of fixed concentration of thrombin into the brush for another hour. The absorbance reading was obtained after aptamer functionalization and thrombin introduction. The UV spectra are plotted and compared. A subtle two-peak absorbance was observed at 581 and 631 $\mathrm{nm}$ in comparison to the initial loading absorbance peak at $582 \mathrm{~nm}$ upon addition of TBA. As the brush is capable to re-swell in the wet state, the particles would move freely in the brush. TBA that interacts with the AuNPs in the brush by chemisorption and physisorption causes the particles to move closer together and form huge clusters of AuNPs, giving rise to the double absorbance peak. Upon collapse of the brush, the free end chains will again, surround the particles, allowing most of the particles to remain in the brush. This is supported by FESEM images where huge clusters are observed after the addition of aptamer into the polymer matrix (Figures 6-11A \& B). 

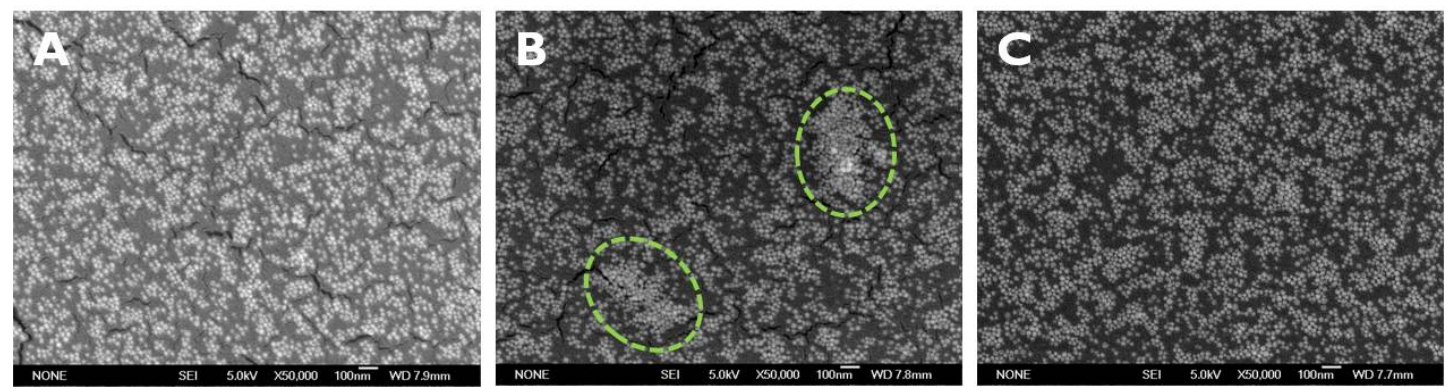

Figure 6-11. FESEM images of AuNP-POEGMA (A) after assembly, (B) postaptamerization and (C) afer detection of $100 \mathrm{ng} / \mathrm{mL}$ thrombin in binding buffer solution.

To study the effect of functionalization time on the absorbance peak, real time in $0.1 \mu \mathrm{M}$ TBA was conducted on 2 rounds POEGMA brush loaded with AuNPs for 1 hour. The absorbance reading is measured at every 1 min interval in solvent. We observed the evolution of the absorbance spectra from one distinct peak to two peaks (i.e. sharp peak at $537 \mathrm{~nm}$ and shoulder at $624 \mathrm{~nm}$ ), This is to be expected since the particles tend to be pulled closer together as TBA binds to the AuNPs over time. As a result, the particles will slowly form large clusters, giving rise to two absorbance peaks. Surprisingly, when the brush is dried, the absorbance intensity of the two peaks are reversed. The peak at $537 \mathrm{~nm}$, the lower wavelength is now slightly lower than at the higher wavelength, $624 \mathrm{~nm}$. This phenomenon is likely possible because in the wet state, the polymer chains swells and the AuNPs are well-dispersed. This is confirmed by the presence of the high single peak. Nonetheless, the presence of shoulder indicates that clusters are gradually forming. Upon collapse, the single nanoparticles become more closely packed and thus, contribute to an increase in absorbance peak at higher wavelength.

After successful aptamer functionalization, we proceeded to detect thrombin to confirm the operability of our sensing mechanism. In this case, we initially tested our 
system by detecting $100 \mathrm{ng} / \mathrm{mL}$ of thrombin in thrombin binding buffer using substrate coated with 2 rounds POEGMA. The UV-vis absorbance was obtained.
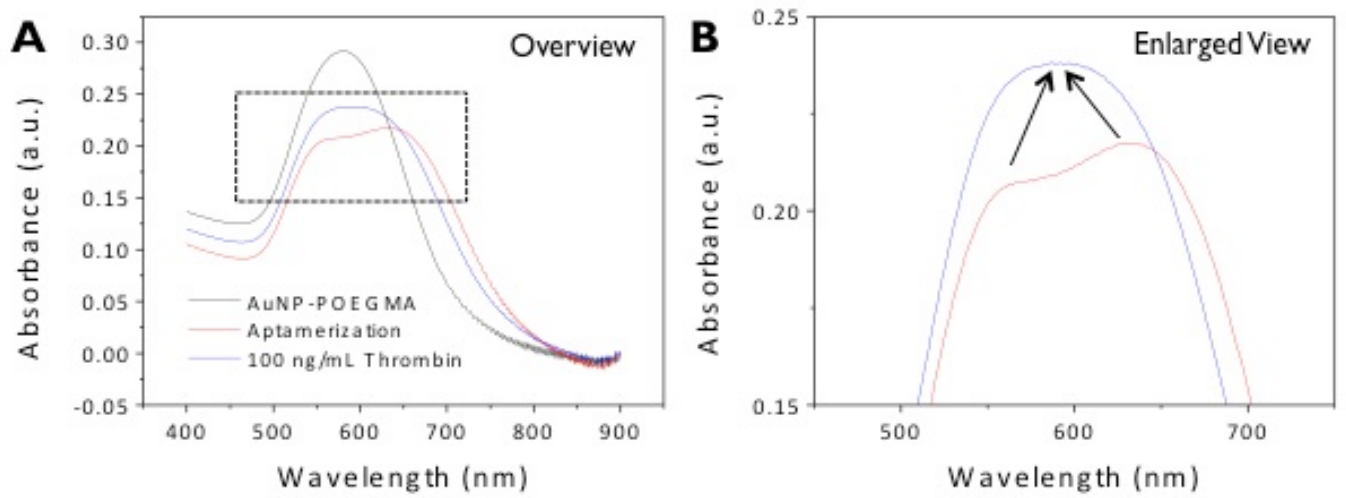

Figure 6-12. (A) UV-vis spectra of AuNP-POEGMA after assembly (black trace), after aptamerization (red trace) and after detection of $100 \mathrm{ng} / \mathrm{mL}$ thrombin in binding buffer solution. (B) Enlarged view of the marked region in (A).

Surprisingly, the subtle two peaks diminished into a single peak and blue shift to $597 \mathrm{~nm}$ as shown in Figure 6-12. This phenomenon is possibly because in presence of thrombin, TBA will form a folded configuration, weakening the physical adsorption to gold surface. Due to the strong affinity of the TBA to thrombin molecule, TBA will then bind to thrombin. As a result, the nanoparticles are again mobile and able to break away from one another, leaving single nanoparticles in the brush. Upon collapse of the brush, the single AuNPs are protected by the long free end chains, making it appear dispersed once again. This could be seen from the formation of the single peak, supporting our point that huge clusters are no longer present.

Real time in $100 \mathrm{ng} / \mathrm{mL}$ thrombin was also conducted on 2 rounds POEGMA brush loaded with AuNPs for one hour. The absorbance reading is measured after every $1 \mathrm{~min}$ interval in the wet state. We could clearly see the shoulder disappearing at $650 \mathrm{~nm}$ to a more distinct absorbance peak at $544 \mathrm{~nm}$. This is to be expected since the 
physisorption interaction between the TBA and the AuNPs are surpassed by the strong binding affinity of TBA to thrombin molecules. Since TBA forms a folded configuration with thrombin, the AuNPs will break away from one another over time, forming dispersed nanoparticles. Upon collapse, the double peak disappears, leaving a single peak as shown in the spectral change upon drying. FESEM images (Figures 611B \& C) also prove this the huge clusters which appear during functionalization is no longer present after the addition of thrombin thus proving, our hypothesis.

To verify that high loading is essential for this phenomenon to occur, another substrate polymerized with 2 rounds POEGMA brush was immersed in $18 \mathrm{~nm}$ AuNPs overnight but of lower concentration. Similarly, the substrate was immersed in $0.1 \mu \mathrm{M}$ TBA for 1 hour, followed by $10 \mathrm{ng} / \mathrm{mL}$ thrombin. The absorbance reading is recorded after loading, aptamerization and thrombin introduction. There was no visible peak shift upon addition of aptamer. This is due insufficient nanoparticles being far apart from one another. Though TBA can covalently bind to AuNPs, the high inter-particle distance between the particles prevents physisorption of the TBA tail with other AuNPs. As a result, clustering of nanoparticles with one another is not possible. In addition, the chemisorbed TBA may form a very strong covalent gold-thiol bond with the AuNPs such that some AuNPs may be displaced from the brush into the solution, causing the intensity to be reduced. Due to the absence of clustering upon aptamer functionalization, de-aggregation of AuNPs will thus, be impossible. This thus proves that high loading is in fact, necessary to initiate this aggregation/de-aggregation of AuNPs phenomenon in the polymer brush.

To further justify the sensitivity of our proposed sensing platform, the substrates of AuNP in 2 rounds of POEGMA brush were immersed in varying concentrations of thrombin stock solution diluted with thrombin binding buffer, ranging from 1.00 
$\mathrm{pg} / \mathrm{mL}$ to $100 \mathrm{ng} / \mathrm{mL}$. The responses were quantitatively determined based on the blue peak wavelength shift of the TBA upon binding with thrombin. From Figure 6-13, it could be seen that the spectral shift decreases upon addition of lower concentrations of thrombin. Extremely small standard deviations at each thrombin concentration were obtained, exhibiting highly consistent responses. As a result, a dynamic range of 100 $\mathrm{pg} / \mathrm{mL}$ to $10 \mathrm{ng} / \mathrm{mL}$ (2.8 $\mathrm{pM}$ to $0.28 \mathrm{nM})$ was obtained from a sigmoidal calibration plot. Also, based on an experimental signal to noise ratio $(\mathrm{S} / \mathrm{N})$ of 3 , LOD of 23.5 $\mathrm{pg} / \mathrm{mL}(0.64 \mathrm{pM})$ with a correlation factor of 0.99 was achieved, which is significantly better than other LSPR sensors recently reported ${ }^{285,289,290}$.
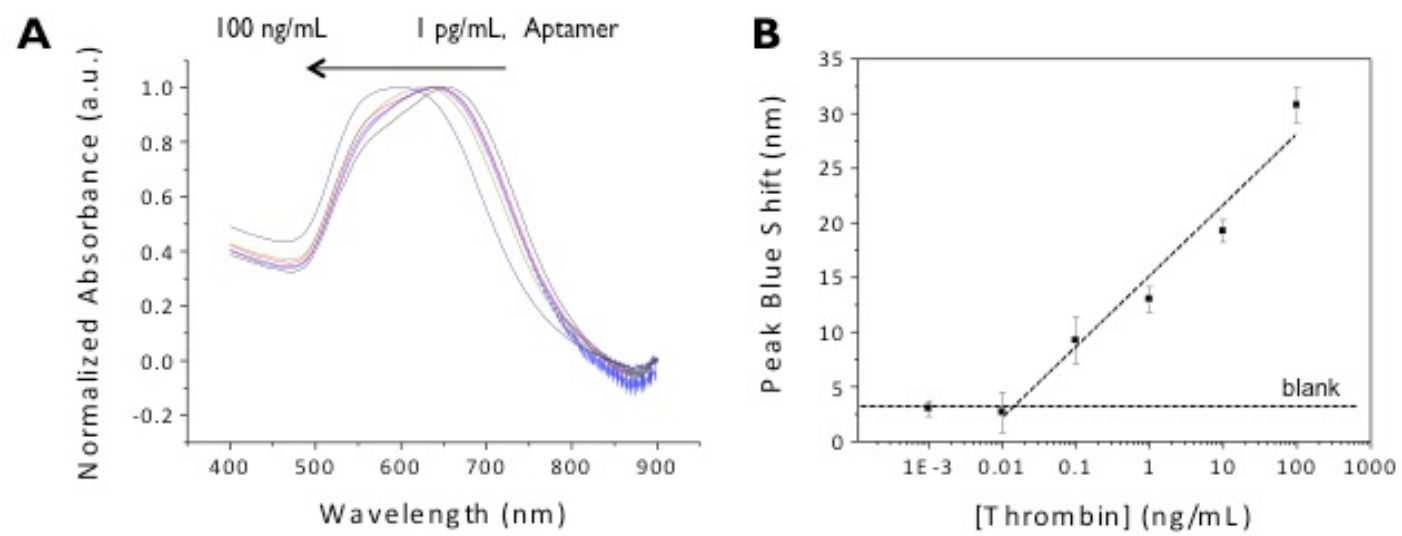

Figure 6-13. (A) UV-vis spectra of AuNP-POEGMA after detection of thrombin in binding buffer solution with concentration ranging from $1 \mathrm{pg} / \mathrm{mL}$ to $100 \mathrm{ng} / \mathrm{mL}$. (B) Response curve generated from the UV-vis absorbance blue shift against thrombin concentration.

With respect to thrombin detection, our limit-of-detection surpassess state-of-theart technologies currently available on the market, which are based on enzyme-linked immunosorbent assay (ELISA) techniques. ${ }^{291-293}$ A comparison with other developed methods, both commercially available ones as well as those recently developed in other labs, is presented in Table 6-1. 
Table 6-1. A detection performance comparison of this work with other techniques, both commercially available and recently developed in other labs.

\begin{tabular}{|c|c|c|c|}
\hline \multicolumn{4}{|c|}{ Commercially Available } \\
\hline Manufacturer / Product Name & $\begin{array}{l}\text { Limit-of- } \\
\text { Detection }\end{array}$ & Linear Range & Ref. \\
\hline $\begin{array}{l}\text { Abcam / Thrombin (Factor II) Human } \\
\text { ELISA Kit }\end{array}$ & $0.3 \mathrm{ng} / \mathrm{mL}$ & $0.313 \mathrm{ng} / \mathrm{mL}-20 \mathrm{ng} / \mathrm{mL}$ & 291 \\
\hline $\begin{array}{l}\text { Anaspec / SensoLyte }{ }^{\circledR} 520 \text { Thrombin } \\
\text { Activity Assay Kit*Fluorimetric* }\end{array}$ & $6.25 \mathrm{ng} / \mathrm{mL}$ & No data & 292 \\
\hline Abnova / Thrombin (Human) ELISA Kit & $0.16 \mathrm{ng} / \mathrm{mL}$ & $0.313 \mathrm{ng} / \mathrm{mL}-20 \mathrm{ng} / \mathrm{mL}$ & 293 \\
\hline \multicolumn{4}{|c|}{ Recently Developed in Labs } \\
\hline Detection Technique & $\begin{array}{l}\text { Limit-of- } \\
\text { Detection }\end{array}$ & Linear Range & Ref. \\
\hline $\begin{array}{l}\text { Aptamer-based surface enhanced Resonance } \\
\text { Raman scattering (SERRS) }\end{array}$ & $\begin{array}{l}3.67 \mathrm{ng} / \mathrm{mL} \\
(100 \mathrm{pM})\end{array}$ & No data & 285 \\
\hline $\begin{array}{l}\text { Aptamer-based sandwich assay with } \\
\text { magnetic beads and quantum dots }\end{array}$ & $10 \mathrm{ng} / \mathrm{mL}$ & $100 \mathrm{ng} / \mathrm{mL}-1000 \mathrm{ng} / \mathrm{mL}$ & 290 \\
\hline Aptamer-based impedimetry & $\begin{array}{l}0.16 \mathrm{ng} / \mathrm{mL} \\
(4.4 \mathrm{pM})\end{array}$ & $\begin{array}{l}0.17 \mathrm{ng} / \mathrm{mL}-18.36 \mathrm{ng} / \mathrm{mL} \\
(4.7 \mathrm{pM}-500 \mathrm{pM})\end{array}$ & 289 \\
\hline $\begin{array}{l}\text { Aptamer-based catalyst dependent } \\
\text { electrochemistry }\end{array}$ & $\begin{array}{l}0.73 \mathrm{ng} / \mathrm{mL} \\
(0.02 \mathrm{nM})\end{array}$ & $\begin{array}{l}3.67 \mathrm{ng} / \mathrm{mL}-918 \mathrm{ng} / \mathrm{mL} \\
(0.1 \mathrm{nM}-25 \mathrm{nM})\end{array}$ & 294 \\
\hline $\begin{array}{l}\text { Aptamer-based metal enhanced } \\
\text { fluorescence }\end{array}$ & $\begin{array}{l}2.6 \mathrm{ng} / \mathrm{mL} \\
(0.073 \mathrm{nM})\end{array}$ & $\begin{array}{l}3.67 \mathrm{ng} / \mathrm{mL}-3.67 \mu \mathrm{g} / \mathrm{mL} \\
(0.1 \mathrm{nM}-100 \mathrm{nM})\end{array}$ & 295 \\
\hline $\begin{array}{l}\text { Aptamer-based AuNP de-aggregation on } \\
\text { solid substrate }\end{array}$ & $23.5 \mathrm{pg} / \mathrm{mL}$ & $100 \mathrm{pg} / \mathrm{mL}-10 \mathrm{ng} / \mathrm{mL}$ & $\begin{array}{l}\text { This } \\
\text { work }\end{array}$ \\
\hline
\end{tabular}

\subsection{Conclusion}

The incorporation of non-functionalized AuNPs into unmodified POEGMAcoated substrates reported herein exemplifies a new and facile technique to achieve high nanoparticle loading in polymer brushes of high grafting densities in a controlled manner. This technique, referred to as in-stacking, can simply be achieved via repeated cycles of immersion-drying-re-immersion in nanoparticle solution. As we show here that in-stacking is feasible without any chemical modification, we foresee 
that this strategy can be extended extensively for the incorporation of other nanoparticles into polymer brushes especially if coupled with covalent attachment schemes.

We have also offered a facile method in attaining a rough surface of the brush by polymerizing 2 rounds POEGMA brush simply by reversing the water/alcohol solvent ratio (i.e. 1st round polymerized under $3 \mathrm{~h}$ POEGMA in water/methanol solvent ratio $(20: 80 \mathrm{v} / \mathrm{v})$ and 2 nd round under $6 \mathrm{~h}$ POEGMA in water/methanol solvent ratio (80:20 $\mathrm{v} / \mathrm{v})$ ). We also tested our novel platform for the detection of thrombin in a solid-phase interface by exploiting the nanomobility of the AuNPs in the brush, as well as, the surface roughness attained under 2 rounds POEGMA. This was conducted by first loading unmodified AuNPs into POEGMA brush via simple immersion, followed by functionalization of AuNPs with thrombin binding aptamer and subsequently by detection of thrombin based on aggregation/de-aggregation of the AuNPs. The detection performance was proven to be significantly better than many conventional studies whereby our limit of detection was found to be as low as $0.64 \mathrm{pM}$ with a wide dynamic range of $100 \mathrm{pg} / \mathrm{mL}$ to $10 \mathrm{ng} / \mathrm{mL}$ and a correlation factor of 0.99 . Such advances offer high prospects in attaining a simple, economical and highly-sensitive biosensing platform for point-of-care use. 


\section{Chapter 7}

\section{Effects of Ionic Strength and Surfactant Concentration on Surface Adsorption of Gold Nanorods}

\subsection{Introduction}

Gold nanoparticles have recently attracted much attention due to their shape- and size-dependent optical properties originating from localized surface plasmon resonance (LSPR). Out of the many types of nanoparticles ${ }^{296-299}$, gold nanorods (AuNRs) are especially attractive owing to their unique spectroscopic properties related to a tunable aspect ratio and relatively high sensitivity ${ }^{300}$. There have been several efforts to detect biological entities using AuNRs in solution ${ }^{301-304}$. However, in order to develop a convenient and reliable diagnostic device, it is highly desirable to assemble the nanorods on surfaces of appropriate substrates ${ }^{19,305,306}$.

With respect to the deposition and immobilization of AuNRs on a substrate

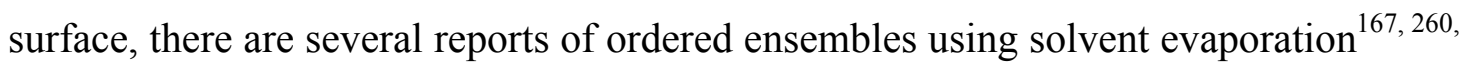
307-309. Although it is generally possible to attain assemblies with highly ordered morphologies and high density through solvent evaporation, it may be neither straightforward to coat large area surfaces nor entirely convenient as the atmospheric conditions must be strictly controlled in order to attain a consistent evaporation rate ${ }^{310}$. In this report, we adopt a full-immersion method for convenient coating of large areas of substrates in a much more predictable and uniformed fashion. These types of assemblies offer high surface areas and better access for the attachment of biological moieties, which subsequently result in better recognition and detection capabilities. 
Although there are many reports on ionic strength-induced flocculation ${ }^{165,311,312}$, there has been limited discussion on the effects of varying ionic strength on the surface assembly of AuNRs on fully immersed substrates. In a recent report, Sethi et al. have presented a study on the stability and aggregation of AuNRs with respect to ionic strength in biological-based solutions ${ }^{313}$. Although there are several fundamental similarities between self-assembly in solution and surface assembly, findings from a detailed study of surface assembly are essential to provide more relevant references for the development of practical and clinically useful biosensors. Apart from ionic strength, the surfactant concentration also represents another key determining factor in surface assembly. While it was briefly reported by Nikoobakht et al. that high concentrations of surfactant used in their half-insertion method may cause unwanted organic crystallites ${ }^{312}$, such issues are uniquely relevant to self-assembly methods based on solvent evaporation. Furthermore, the minimum surfactant concentration required to induce unwanted crystallization was not specifically mentioned in their report. In almost all recently published works, the CTAB concentration is kept minimum before any attempt of surfacial assembly ${ }^{314,315}$.

In this report, we varied not only the ionic strength but also the CTAB concentration and studied their coinfluence in solution as well as the effect on assembly based on the full immersion method. We present an alternative mechanism which shows the existence of a two-tiered shielding effect in nanorod solution at slightly higher surfactant concentration, which would otherwise bemissed in experimentations conducted at single surfactant concentration. By varying the ionic strength at three fixed CTAB concentrations of the AuNR solution, we have achieved tunable densities of AuNR assemblies. We conclude that the dynamics of internanorod 
and nanorod-substrate interactions leading to surface assembly may differ greatly according to surfactant concentration.

Understanding such interplay of dual interactions is essential in attaining an optimal density of AuNRs not only in assemblies but also in other applications such as tuning electrostatic bioconjugation of biomolecules to AuNRs. The occurrence of a two-tiered shielding effect may also offer a more direct electrostatic-based nanoparticle functionalization pathway where, instead of sequentially modifying the surface with molecules of alternating charges, a solution containing a mixture of charged molecules at adjusted concentrations may be introduced in a single step. As the general concepts presented herein can be extended to the use of a whole range of other salts and surfactants, their significance remains relevant for works involving electrostatically stabilized colloid solutions.

\subsection{Specific Methods}

\subsubsection{Formation of APTS-PSS layer}

The glass coverslips were precleaned in a $3: 1 \mathrm{H}_{2} \mathrm{SO}_{4} / \mathrm{H}_{2} \mathrm{O}_{2}$ piranha solution for 30 min. After rinsing with DI $\mathrm{H}_{2} \mathrm{O}$ and drying under nitrogen, the cleaned coverslips were immersed in aminopropyltriethoxysilane (10\%) in ethanol for $1 \mathrm{~h}$ at room temperature. They were, then, sonicated and rinsed with ethanol and cured at $120{ }^{\circ} \mathrm{C}$ for $1 \mathrm{~h}$. They were finally immersed in a solution of $20 \mathrm{mg} / \mathrm{mL}$ PSS for $1 \mathrm{~h}$, rinsed with DI water, and blown dry with nitrogen.

\subsubsection{Surface assemble of gold nanorods}

In the full immersion method, the modified coverslips were completely immersed in the AuNR solution for $18 \mathrm{~h}$ at room temperature. Thereafter, the coverslips were rinsed copiously with DI water (to remove unbound AuNRs) and dried under flowing 
nitrogen. Control experiments were also performed by immersing unmodified coverslips (precleaned and bare) and APTS-functionalized coverslips in nanorod solution. In the solvent evaporation method, the AuNR solution was directly pipetted onto precleaned unmodified coverslips, and the droplets evaporated overnight in the oven at $60^{\circ} \mathrm{C}$.

\subsubsection{Gold nanorod surface assembly retention study}

The glass coverslips were immersed in a solution of phosphate buffered saline (PBS) at $\mathrm{pH} 7.4$ to simulate conditions in real biological applications and subjected to orbital shaking for $1 \mathrm{~h}$. After that, the coverslips were thoroughly rinsed with DI water and dried under nitrogen before reobtaining its UV absorbance spectra. This washing procedure was repeated for the retention test.

\subsubsection{Characterization}

AuNRs were characterized by their UV-vis absorbance, ל-potential, and particle size prior to assembly. AuNR assemblies were characterized by their UV-vis absorbance and SEM visualization. Solution-based $\zeta$-potential and particle size measurements were obtained using a $\zeta$-potential analyzer (Nano-ZS Zetasizer with DTS Nano Software, Malvern Instruments, Malvern, UK). All UV spectra were obtained using a UV spectrophotometer (UV-2540, Shimadzu, Kyoto, Japan) while SEM visualization was performed using FESEM microscope (JEOL JSM-6700F, Tokyo, Japan).

\subsection{Results and Discussion}

The strategy adopted in this report for the deposition of AuNRs is based on electrostatic interactions between the positively charged CTAB molecules surrounding 
the AuNRs and the negatively charged surface due to PSS. The deposition scheme is demonstrated in Figure 7-1. In order to achieve a net negative charge on the glass substrate, it was first treated with APTS. The APTS SAM represented a uniform positively charged base layer. The utilization of a negatively charged strong polyelectrolyte, PSS, enabled a convenient yet complete charge reversal of the surface. In order to minimize complications in demonstrating the effects of ionic strength on the electrostatic assembly of AuNRs, we allowed the AuNR solution to remain in its native surfactant, CTAB, without any modifications or surfactant exchanges.

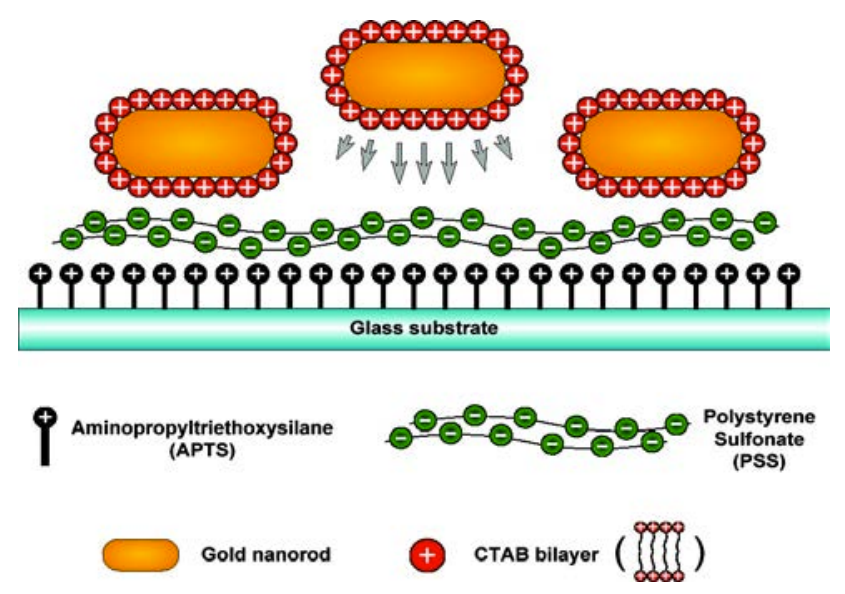

Figure 7-1. Schematic representation of CTAB-protected AuNR deposition on PSS-APTS-glass surface based on electrostatic interactions. Reprinted with permission from Langmuir, 2010, 26 (14), pp 12433-12442. Copyright 2010 American Chemical Society.

Contrary to initial supposition that AuNRs, capped with a bilayer of positively charged CTAB molecules, will readily deposit on a negatively charged surface of fully immersed substrate, nearly zero deposition has been observed. Deposition of AuNRs was achieved only when salt was gradually added. It was later found that the adjustment of ionic strength within a specific range may advantageously affect the density of surface assembly of AuNRs. This favorable effect of ionic strength also 
varies under different $\mathrm{CTAB}$ concentrations. Motivated by this interesting result, we study the dynamics of internanorod and nanorod-substrate interactions that eventually allow us to tune the surface density of AuNR assembly via adjustment of ionic strength.

Kawamura and co-workers have recently observed that the surfactant concentration played a critical role in the assembly of AuNRs both in solution and on substrates after drying ${ }^{308}$. They varied the concentration by subjecting the nanorod solution to two and three rounds of centrifugation, each time with redispersion in Milli-Q water, and found that the 2-D assembly configuration changed from a densely aligned, parallel assembly to a loosely aligned, head-to-tail assembly. Since three rounds of centrifugation may risk deformation of AuNRs or "crashing out" of solution $^{313}$, we only subjected our nanorod solutions to two rounds of centrifugation. A large portion of excess $\mathrm{CTAB}$ is removed in the first round by completely removing the surfactant supernate by careful pipetting and then redispersing in DI water. The solution is then equally divided into three parts before the second round of centrifugation in order to obtain different final CTAB concentrations. After the second centrifugation, the supernate of each of the three parts is (1) diluted 5 times, (2) diluted 10 times, and (3) completely substituted with DI water. For the purpose of this report, we identify the respective resultant CTAB concentrations as (1) high, (2) moderate, and (3) low. The AuNR concentrations of the three parts are equal.

The UV spectra of AuNR solutions (Figures 7-2A-C) show that, besides the case of low $\mathrm{CTAB}$ concentration where severe aggregation starts to occur at $60 \mathrm{mM}$, consistent transverse and longitudinal peaks are observed with a general trend of initial decrease followed by subsequent reattainment of absorption intensities with increasing ionic strength. While peak shifting induced by different salt concentrations has been 
reported in AuNR solutions due to aggregation in a side-by-side arrangement ${ }^{115}$, only reduction in absorption intensities at consistent transverse and longitudinal peak positions is observed here as a result of slight aggregation without any specific arrangement. At low $\mathrm{CTAB}$ concentration and high ionic strength, severe aggregation occurs as indicated by diminished peaks. The UV spectra of AuNR assemblies (Figures 7-2D-F) show a subtle shoulder at ca. $530 \mathrm{~nm}$ instead of a distinct peak. Unlike in solution, the nanorods are now confined in two dimensional space. As they are deposited horizontally, the transverse peak becomes diminished, and there is no direct correlation with the surface density. Since the transverse peak is also much less sensitive to local environmental changes and hence not relevant for biodetection applications, we will focus our discussion on the longitudinal peak. A plot of longitudinal peak position depending on salt concentration is presented in Figure 72G. The intensity of absorbance, in general, corresponds to the density of the assembled nanorods. Under moderate and high CTAB concentration, no distinct peak was detectable at zero salt concentration, which correlates to almost zero or minute amount of deposition of nanorods. The intensity of longitudinal peak increased with increasing salt concentration until it reached an absorbance of ca. 0.90 arbitrary units before it began to fall off, following a parabolic trend. This, however, was not observable for AuNR solutions at low CTAB concentrations. The addition of salt only yielded significant enhancement of absorbance over a much narrower range of ionic strength (up to $40 \mathrm{mM}$ ) until the maximum absorbance is reached. Further increment of salt beyond this point resulted in zero observable absorbance. Unlike high and moderate $\mathrm{CTAB}$ concentration, small, yet clear absorbance with a distinct peak was observed, even without any salt addition. Although there is also a slight increase in absorbance at zero salt concentration shown in Figure 7-2E, there is no distinct peak, 
and no peak value was detected by the spectrophotometer. Since the solution is prepared and left to stand prior to the immersion of substrates, we presume that at any given salt and CTAB concentration the AuNR solution will inevitably first attempt to reach maximum stability through the reduction of nanorod Gibbs free energies before the nanorods begin to assemble on the surface. Hence, for the purpose of this discussion, we will qualitatively explain the observed overall trend shown in Figure 72 in two inter-related parts by considering (1) the nanorod-nanorod interactions prior to substrate immersion and (2) the nanorod-subsrate interactions. 
A

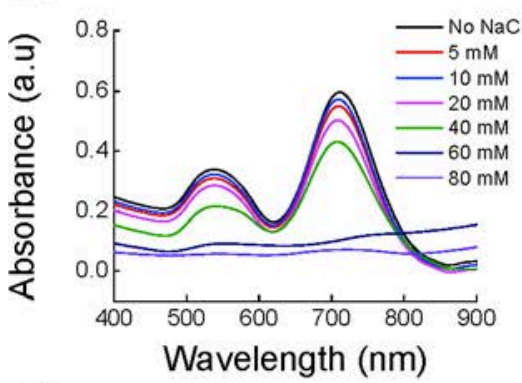

D

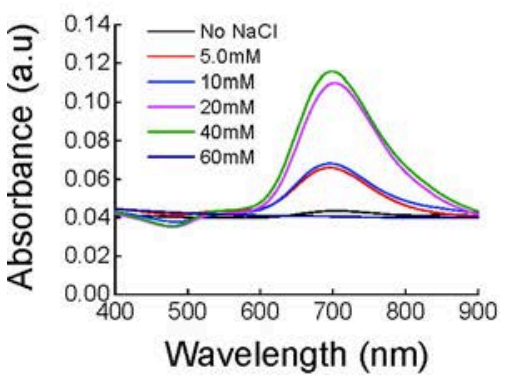

B

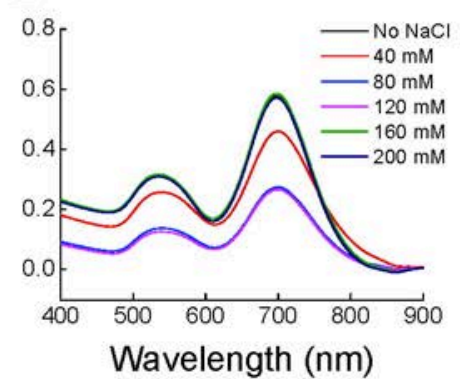

E

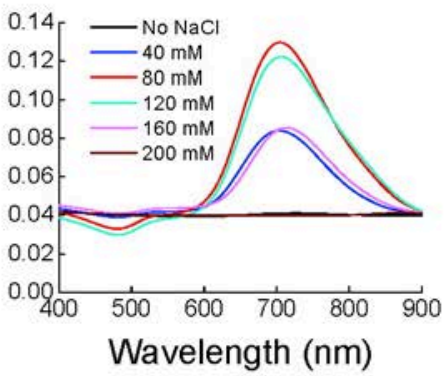

C

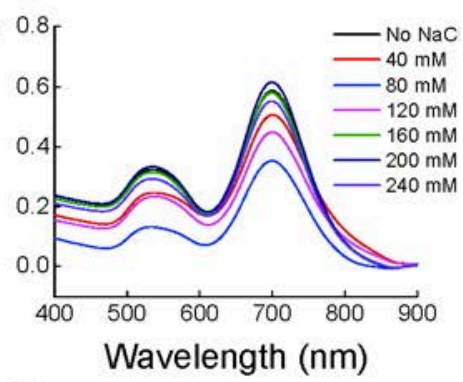

$\mathbf{F}$

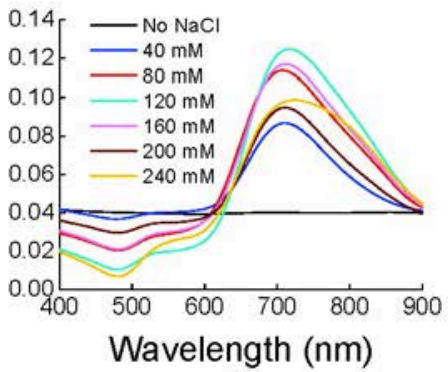

G

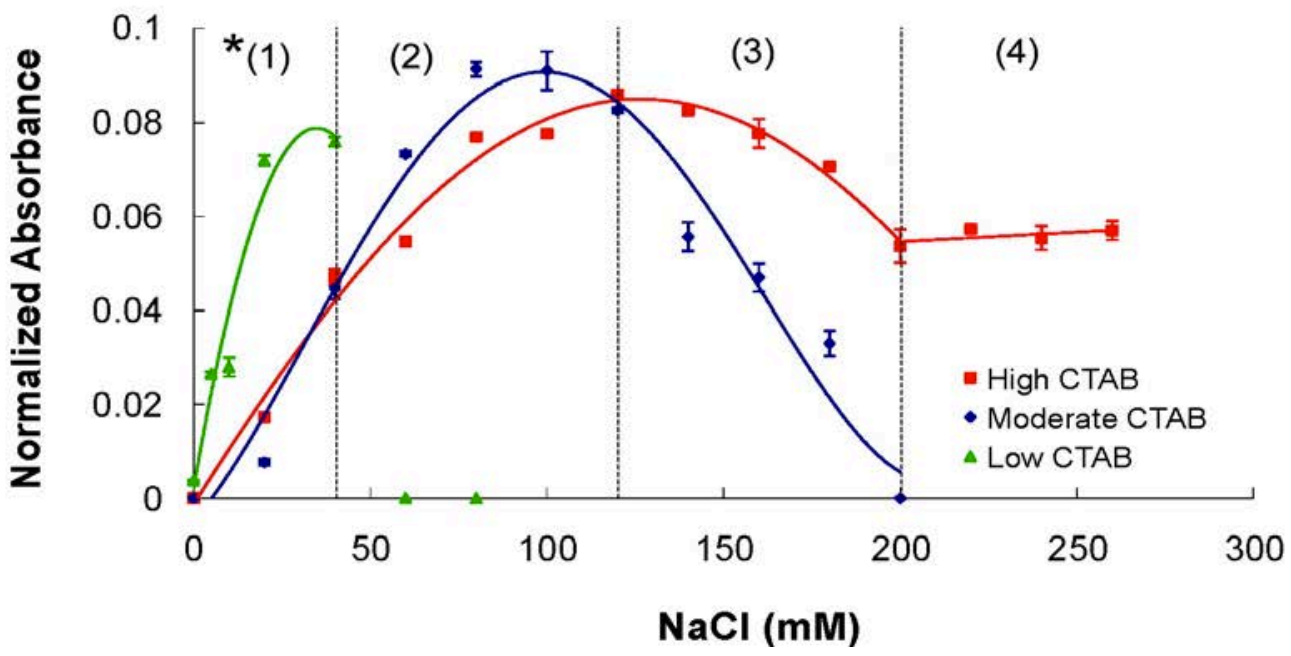

Figure 7-2. UV-vis spectra of AuNR solutions with (A) low, (B) moderate, and (C) high $\mathrm{CTAB}$ concentrations at different $\mathrm{NaCl}$ concentrations. UV-vis spectra of GNR assemblies on glass from a solution with (D) low, (E) moderate, and (F) high CTAB concentrations at different $\mathrm{NaCl}$ concentrations. (G) Correlation between UV-vis absorbance and $\mathrm{NaCl}$ concentration. *The numbers in parentheses indicate the different regions of ionic strength as referred to in this discussion. Error bars represent standard deviation. Reprinted with permission from Langmuir, 2010, 26 (14), pp 12433-12442. Copyright 2010 American Chemical Society. 
Nanorod-nanorod interactions represent one of several determining factors in the surface assembly of nanorods. From Figure 7-2, it is clear that the introduction of salt allows for the mediation of assembly through anions present in the solution (in this case $\mathrm{Cl}^{-}$). Since the AuNR solution can be modeled as a colloidal system relying on electrostatic stability, we can discuss the observed phenomena in assigned ionic strength regions of Figure 7-2G based on the Derjaguin, Landau, Verway, and Overbreek (DLVO) theory of colloidal stability ${ }^{316}$. Briefly, this theory suggests that the resultant force between charged surfaces in a liquid medium is due to a combined effect of van der Waals attraction and electrostatic repulsion due to a double layer of counterions $^{317}$.

\subsubsection{Two-tiered shielding effect and general parabolic trend in surface} density

In a process described as electrostatic shielding, anions from added salt form a double-layer structure and modulate nanorod-nanorod interactions. In the absence of salt, regardless of CTAB concentration, a repulsive force between the nanorods exists due to the exposed, positively charged CTAB bilayer capping the nanorods, inhibiting assembly. With gradual addition of salt, double-layer structure around the cationic charges (CTAB) of the nanorod surface is formed, namely first shielding effect, negatively charged as shown in Figure 7-3. When double-layer forces of repulsion between nanorods become less dominating compared to the attractive van der Waals forces, slight aggregation begins to occur in solution, indicating reduced stability (as evident from the general reduction of absorption intensities in Figures 7-2A-C. Consequently, assembly becomes more favorable. This is experimentally evident from regions 1 and 2 of Figure 7-2G. Such assembly of AuNRs occurs until it reaches a 
point when it is sufficient to allow for the adsorption of either free salt cations (in this case, $\mathrm{Na}^{+}$) or excess CTAB molecules which had earlier remained unbound. We believe that in our considerably dilute aqueous environment CTAB molecules were more favorable to be adsorbed on the double layer of the GNRs than free-Na+, which has higher affinity for water ${ }^{318,319}$. As the surface potential becomes more negative due to the increase in salt concentration, CTAB molecules surround the nanorod in a layer-by-layer fashion, namely second shielding effect, positively charged, as shown in Figure 7-3. This eventually reintroduces a repulsive force between the nanorods, restabilizing the solution (as evident from the general reattainment of absorption intensities in Figures 7-2B, C) and inhibiting assembly. This assembly inhibition is reflected in regions 3 and 4 of Figure 7-2G; onset of the assembly inhibition occurs at $\mathrm{NaCl}$ concentration of 100 and $120 \mathrm{mM}$ for the moderate and high concentration of $\mathrm{CTAB}$, respectively. It was not observed in the case of low CTAB concentration due to insufficient $\mathrm{CTAB}$ molecules to form the second shielding effect, consequently leading to nanorod aggregation beyond $\mathrm{NaCl}$ concentration of $40 \mathrm{mM}$ as shown in Figure 7-2A. At moderate $\mathrm{CTAB}$ concentration, no surface assembly is observable when the AuNRs eventually acquire a completely dominating double-layer repulsive force, i.e., second shielding layer, to strongly repel each other. As shown in Figure 72G, this occurs at $\mathrm{NaCl}$ concentration of $200 \mathrm{mM}$. This is in contrast to high CTAB concentration where surface assembly is still observable at $\mathrm{NaCl}$ concentration of 200 $\mathrm{mM}$ and beyond, suggesting less effective double-layer repulsion. 


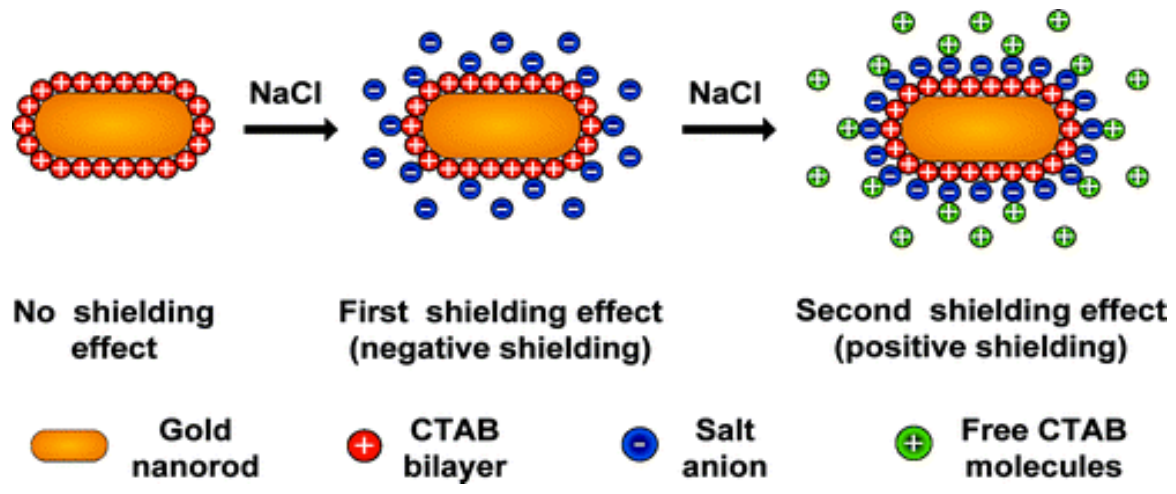

Figure 7-3. Schematic illustration of CTAB-protected gold nanorod experiencing negative and positive electrostatic shielding effects with increasing ionic strength at a fixed moderate or high CTAB concentration. The relative sizes of the gold nanorod, CTAB bilayer, salt anion and free CTAB molecule are not to scale. The free CTAB molecule presented here is a simplified representation. In actual fact, it could be in the form of micelles or rod-shaped bilayers. Reprinted with permission from Langmuir, 2010, 26 (14), pp 12433-12442. Copyright 2010 American Chemical Society.

Sethi and co-workers ${ }^{313}$ observed a similar trend in their solution-based assembly of nanorods when the salt concentration of nanorod solution was varied at a single concentration of CTAB. No aggregation was observable at low salt concentrations. As they increased the salt concentration, aggregation became observable. Upon further increase, disaggregation occurs. However, in our case of moderate to high CTAB concentrations, no severe aggregation is observed in our nanorod solutions under all salt concentrations possibly due to the higher concentration of CTAB than the one used by Sethi.

7.3.2 Charge coverage around gold nanorods from peak $\mathrm{NaCl}$ concentration onwards

In terms of nanorod-CTAB/anion/free-CTAB interactions at peak $\mathrm{NaCl}$ concentration and beyond, i.e., regions 3 and 4 in Figure 7-2G, it was initially unclear 
the level of anion coverage on the AuNRs due to spatial and electrostatic constraints. If there is already a complete coverage of anions around the GNRs at peak $\mathrm{NaCl}$ concentration, free-CTAB molecules would immediately form the second shielding layer and gradually deplete with further salt addition as excess anions draw CTAB molecules away from the second shielding layer. Alternatively, if complete coverage is yet to be achieved, the number of anions surrounding the AuNRs will continue to increase after the peak $\mathrm{NaCl}$ concentration is reached. In this case, free-CTAB molecules would begin to form the second shielding layer at peak $\mathrm{NaCl}$ concentration and gradually accumulate as a consequence of the increasing number of anions in the first shielding layer. Both of these probable schemes may result in the general trend observed in region 3 of Figure 7-2G: absorbance decreasing with the addition of salt due to the increasing double-layer repulsive force contributed by either negative or positive charges.

Therefore, further investigation is performed using $\zeta$-potential and dynamic light scattering (DLS) analysis. From Table 7-1, a AuNR solution with moderate CTAB concentration shows a relatively high $\zeta$-potential of $45.3 \pm 2.1 \mathrm{mV}$ in the absence of salt. It decreased to a minimum of $9.9 \pm 0.7 \mathrm{mV}$ at $120 \mathrm{mM} \mathrm{NaCl}$, where maximum absorbance is observed, before starting to increase again. It finally regained a highly positive $\zeta$-potential of $42.3 \pm 1.7 \mathrm{mV}$. The increase in $\zeta$-potential depending on the $\mathrm{NaCl}$ concentration from 120 to $200 \mathrm{mM}$ indicated that there is an accumulation of the outermost positive charges instead of depletion. If depletion occurs, $\zeta$-potential in the repulsive environment would have reached a highly negative value. The only other possible case for an increase in $\zeta$-potential is the depletion of anions from the first shielding layer; which is impossible with increasing ionic strength. 
Table 7-1. ל-Potential and Dynamic Light Scattering (DLS) Size Comparisons for Gold Nanorods Dissolved in Solutions of Different CTAB Concentrations at Various Ionic Strengths ${ }^{a}$

\begin{tabular}{|c|c|c|c|c|c|c|}
\hline \multirow[b]{2}{*}[\mathrm{NaCl}]{$(\mathrm{mM})$} & \multicolumn{2}{|c|}{ low CTAB concn } & \multicolumn{2}{|c|}{ moderate CTAB concn } & \multicolumn{2}{|c|}{ high CTAB concn } \\
\hline & $\zeta$-potential $(\mathrm{mV})$ & size $(\mathrm{nm})$ & $\zeta$-potential $(\mathrm{mV})$ & size $(\mathrm{nm})$ & $\zeta$-potential $(\mathrm{mV})$ & size $(\mathrm{nm})$ \\
\hline 0 & $31.9 \pm 2.5$ & $46.2 \pm 0.2$ & $45.3 \pm 2.1$ & $53.3 \pm 1.0$ & $39.9 \pm 1.5$ & $49.7 \pm 0.8$ \\
\hline 5 & $28.3 \pm 0.1$ & $53.3 \pm 0.2$ & & & & \\
\hline 10 & $22.6 \pm 0.3$ & $55.9 \pm 4.7$ & & & & \\
\hline 20 & $13.0 \pm 2.1$ & $55.9 \pm 1.2$ & & & & \\
\hline 40 & $6.7 \pm 1.0$ & $65.2 \pm 2.0$ & $32.6 \pm 2.0$ & $56.1 \pm 3.1$ & $15.1 \pm 1.4$ & $50.8 \pm 0.4$ \\
\hline 80 & $0.6 \pm 0.3$ & $855.7 \pm 68.6$ & $12.8 \pm 2.0$ & $56.4 \pm 0.2$ & $2.8 \pm 1.3$ & $50.9 \pm 0.4$ \\
\hline 120 & $29.3 \pm 1.5$ & $759.8 \pm 156.3$ & $9.9 \pm 0.7$ & $59.1 \pm 0.4$ & $1.4 \pm 0.6$ & $51.3 \pm 1.8$ \\
\hline 160 & & & $15.7 \pm 2.0$ & $69.5 \pm 0.6$ & $2.6 \pm 0.3$ & $51.9 \pm 1.3$ \\
\hline 200 & & & $42.3 \pm 1.7$ & $91.6 \pm 0.3$ & $20.8 \pm 2.3$ & $54.7 \pm 0.6$ \\
\hline 220 & & & & & $16.3 \pm 0.6$ & $55.2 \pm 1.8$ \\
\hline 240 & & & & & $18.4 \pm 6.3$ & $55.2 \pm 0.4$ \\
\hline
\end{tabular}

${ }^{a}$ Note that the size recorded here is based on dynamic light scattering (DLS) and is, therefore, not representative of the absolute length along any dimension of the rod.

\subsubsection{Modeling second shielding layer as an isolated double layer}

It has long been established that dilute amounts of counterions lead to a thicker double layer and more effective double-layer repulsions as opposed to concentrated amounts of counterions ${ }^{317}$. Hence, the phenomenon of more CTAB molecules resulting in better repulsions, as observed in this study, would appear contradicting. We will therefore explain the mechanistic scheme responsible for the trend in region 3 of Figure 7-2G by considering the second shielding layer as an isolated double layer. Here, anions from the first shielding layer will serve as a hypothetical surface charge underlying an inner Stern layer and an outer diffuse or Guoy layer. As proposed in the previous section, the amount of CTAB forming the second shielding layer at peak $\mathrm{NaCl}$ concentration onward increases with the amount of anions in the first shielding layer. At a fixed moderate CTAB concentration, when ionic strength is increased, more CTAB molecules would be attracted to form the second shielding layer. Part of this population of molecules would form the Stern layer while the rest would be distributed in the Guoy layer. With increasing salt, and therefore a denser hypothetical 
surface charge, more CTAB molecules would be favorably attracted close to the hypothetical surface in an attempt to provide a consistent degree of charge negation and achieve the same resultant surface potential. This would imply lesser amount distributed in the Guoy layer, leading to a thicker overall double layer and eventually better repulsion. Such explanation is not relevant to regions 1 and 2 where the surface charge due to the nanorod-bound CTAB bilayer remains fixed. In region 3 of Figure 7-2G, it is also conversely observed that beyond peak $\mathrm{NaCl}$ concentration the absorbance of nanorod surface assembly obtained from a solution with moderate CTAB concentration falls off much more rapidly than with high CTAB concentration. This is because at a fixed $\mathrm{NaCl}$ concentration the amount of CTAB molecules in the Guoy layer from a solution of moderate CTAB concentration is lower than from a solution of high CTAB concentration. Hence, it is now clear that the scheme is noncontradicting to the theory of double-layer repulsion.

With respect to the increase in $\zeta$-potential for solution of both moderate and high CTAB concentration from 120 to $200 \mathrm{mM} \mathrm{NaCl}$ (Table 7-1), it can be explained that since $\zeta$-potential is measured from the slipping plane, which is considerably closer to the Stern layer, the increase in the number of $\mathrm{CTAB}$ molecules in this region is reflected in the increase of $\zeta$-potential. It is important to note that the $\zeta$-potential does not directly reflect the net charge on the surface but rather represents the potential difference between the dispersion medium and the stationary layer of fluid attached to the dispersed particle ${ }^{320}$. Hence, it can be observed from Table 7-1 that the $\zeta$-potential of the GNR solution with zero salt concentration at moderate CTAB concentration $(45.3 \pm 2.1 \mathrm{mV})$ is higher than that of high CTAB concentration $(39.9 \pm 1.5 \mathrm{mV})$. While the net charge or number of CTAB molecules protecting the nanorods in these two solutions might be relatively close for nanorods with the same surface area, the 
potential difference with reference to the bulk solution is not the same due to the different potential of bulk solutions; high CTAB concentration being more positive results in lower potential difference. Such a phenomenon is usually not observed as colloidal solutions are essentially diluted before measurements. However, in our experiments, we were cautious not to further dilute our solutions due to possibility of ionic dilutions in any of the two shielding layers which may result in inaccurate assessments of the overall charge-shielding trend.

\subsubsection{Trend variation at high CTAB concentration}

At high $\mathrm{CTAB}$ concentration, a unique and interesting trend was observed at 200 $\mathrm{mM} \mathrm{NaCl}$ and above, as shown in region 4 of Figure 7-2G. The absorbance remained notably high, indicating a relatively constant surface density of AuNRs. As discussed earlier, a more concentrated CTAB solution would contribute to a thinner double layer around each AuNR and lesser double-layer repulsive force, eventually leading to higher surface density of assembled nanorods. Therefore, based on our hypothesis, at such high salt and CTAB concentrations, the nanorod would have already been fully covered by adsorbed anions. This layer of anions can serve as a highly dense hypothetical surface where most free CTAB molecules would form a highly compact layer over it. Since the concentration is high, excess CTAB which do not form the compact layer would form a thin Guoy layer, resulting in a thin overall double-layer structure. And since CTAB concentration is fixed, this conformation remains intact even at higher salt concentrations. A gradual increase of surface assembly beyond 200 $\mathrm{mM} \mathrm{NaCl}$ may occur due to excess anions which have not participated in first shielding now participating in a third shielding effect. However, this increase is barely noticeable because of its inefficiency due to the distance from the nanorod surface. 


\subsubsection{Trend variation at low CTAB concentration}

In the absence of salt, the $\zeta$-potential of nanorod solution at low CTAB concentration $(31.9 \pm 2.5 \mathrm{mV})$ is much lower than at moderate and high CTAB concentrations $(45.3 \pm 2.1$ and $39.9 \pm 1.5 \mathrm{mV})$. The deposition of AuNRs even without the addition of salt (Figure 7-4A) is probably due to relatively small amount of CTAB surrounding the AuNR. Because of on/off equilibrium, some CTAB molecules may be desorbed from the surface as the solution is extremely diluted. This is evident as the nanorods start to aggregate at a much lower salt concentration of $40 \mathrm{mM}$. Conversely, when the $\zeta$-potential is at a minimum, the charge on the surface is close to zero. Since there are insufficient free $\mathrm{CTAB}$ molecules to bind to mediating anions, the repulsive forces can be overcome at lower salt concentrations leading to aggregation. This is evident from the $\zeta$-potential and particle size data. The $\zeta$-potential decreases from 31.9 \pm 2.5 to $6.7 \pm 0.1 \mathrm{mV}$. Although there is an increase in $\zeta$-potential between 80 and 120 $\mathrm{mM} \mathrm{NaCl}$, the particle size obtained from the dynamic light scattering (DLS) system indicates aggregation at higher salt concentrations. At 80 and $120 \mathrm{mM} \mathrm{NaCl}$, the particle size recorded is $855.7 \pm 68.6$ and $759.8 \pm 156.3 \mathrm{~nm}$, respectively, as opposed to the normal range of ca. $40-50 \mathrm{~nm}$. It was observed that after a few seconds of standing the solution color changes from reddish-purple to gray. Consequently, surface assembly did not occur due to electrostatic interactions. It was observed that after $18 \mathrm{~h}$ of incubation the glass substrates turns slightly tinted. However, after copious rinsing with DI water, the tint disappears, indicating that it merely originated from the physical adsorption of AuNR aggregates. The UV spectra obtained from the glass substrate also indicated the absence of peaks beyond $60 \mathrm{mM} \mathrm{NaCl}$. Furthermore, we observed that once the AuNRs have been "salted out", it is almost impossible to be 
redispersed. This shows that the resultant CTAB concentration in this case is probably much lower than that used by Sethi ${ }^{313}$.

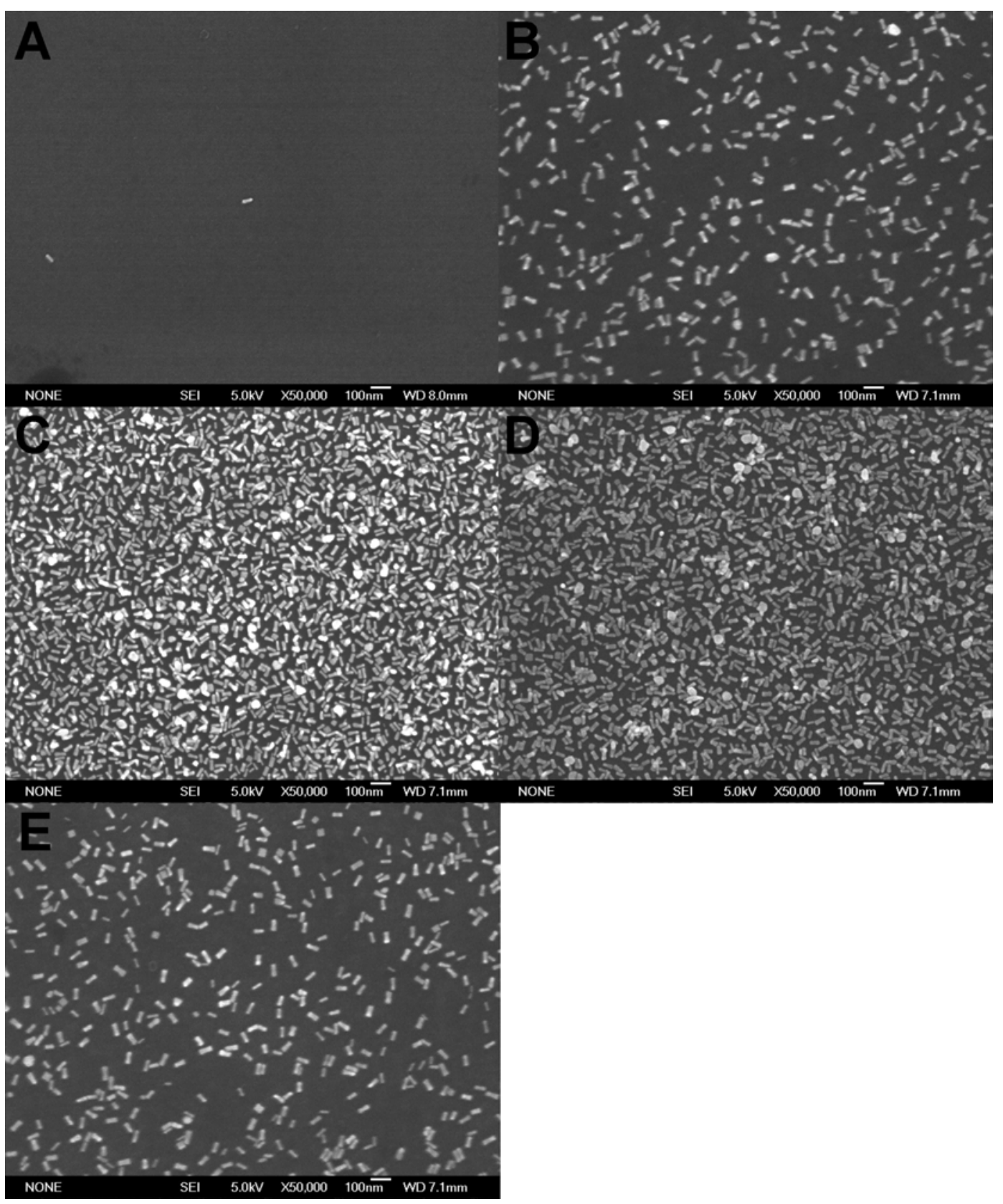

Figure 7-4. SEM images of gold nanorod surface assembly on silicon substrate from a solution of moderate CTAB concentration at (A) 0, (B) 40, (C) 80, (D) 120, and (E) $160 \mathrm{mM} \mathrm{NaCl}$. Note that the sample of moderate CTAB at $200 \mathrm{mM} \mathrm{NaCl}$ is not presented as no AuNR can be located and silicon substrates are used to allow for direct visualization without coating process. Reprinted with permission from Langmuir, 2010, 26 (14), pp 12433-12442. Copyright 2010 American Chemical Society. 


\subsubsection{Nature of CTAB in second shielding layer}

With regard to the second shielding layer, at this juncture, it is not particularly clear, since the CTAB concentration is not determined, whether the CTAB molecules adsorb as singular entities, micelles, or rod-shaped bilayers ${ }^{321}$. In most cases where the concentration is known, this can be determined by comparing against the critical micelle concentration $(\mathrm{cmc})$. While many fundamental works have been performed to study the mechanism behind cationic surfactant adsorption at the solid-liquid interface, they are performed on substrates that are not electrostatically shielded ${ }^{319,322,}$ 323. Since in our case excess CTAB around the nanorod manifests itself as a third electrostatic layer (the first and second being the strongly adhered CTAB bilayer and mediating anions, respectively) and since the nature of adsorbed CTAB is dependent on the interplay of many contributing factors such as surfactant chain length, surface

charge, and degree of displacement of water at the surface ${ }^{321}$, it is beyond the scope of this report to describe in detail the interactions between the various free charged entities which eventually lead to this shielding effect. We are only concerned in determining the existence of a resultant two-tiered shielding effect and studying how it affects the surface assembly of AuNRs.

\subsubsection{Nanorod-substrate interactions}

We have discussed the effects of salt and CTAB concentrations on the stability of AuNRs in solution. Through the DLS size data shown in Table 7-1, throughout the variation of $\mathrm{NaCl}$ concentration, the particle size in solutions remained relatively consistent over the entire experimental range, indicating no sign of aggregation, even at lowest $\zeta$-potentials. At low CTAB concentration, this is valid until $40 \mathrm{mM} \mathrm{NaCl}$, above which severe aggregation occurred (Figure 7-2A). With the latter case being an 
exception, this implied that without an external force self-assembly is not favorable in solution even when the AuNRs are highly electrostatically shielded. Conversely, it implies that electrostatic shielding itself may not necessarily be sufficient to cause surface assembly if without an appropriately treated substrate.

While $\zeta$-potential does not directly indicate the surface charge of the nanorod, we can infer that since the solution is laden with positively charged CTAB molecules, the nanorods would have acquired and maintained a net positive charge for the $\zeta$-potential to remain positive throughout. Therefore, since it is contradictory that a more positively charged particle would be less attracted to a negatively charged substrate, we can deduce that the surface assembly process is more strongly governed by the nanorod-nanorod interactions rather than the nanorod-substrate interactions. In a solution which is highly stabilized by the strong repulsive forces between AuNRs, the attractive force due to the negatively charged substrate surface must be able to overcome the internanorod forces in order for the AuNRs to be attracted to and be deposited on the substrate. In contrast, when repulsive forces have the potential to be easily overcome in solution, the colloidal nanorods become less stable. In such a state, the nanorods will attempt to decrease their surface energy and therefore readily approach others within its proximity. The negatively charged surface can now serve as a convenient template for surface assembly since the force imparted by the surface on a single nanorod now easily overwhelms the forces imparted by other nanorods.

The absorbance of assemblies from solutions with moderate CTAB concentration is lower than one from high $\mathrm{CTAB}$ concentration, as shown in region 1 of Figure 72G. A possible explanation for this is that at moderate to high CTAB concentrations excess CTAB molecules may adsorb on the substrate surface. Such adsorption perhaps occurs more readily at low $\mathrm{NaCl}$ concentration when there are more excess $\mathrm{CTAB}$ 
molecules since the molecules have yet to participate in the second electrostatic shielding. This will render the surface less negative. Hence, at a fixed ionic strength, it is relatively easier, albeit only slightly, for nanoparticles to adsorb at higher CTAB concentration. This can be observed at $\mathrm{NaCl}$ concentrations below $50 \mathrm{mM}$. However, as the particles undergo the first electrostatic shielding and gradually becomes less positive (region 2 of Figure 7-2G), this effect becomes less significant, again indicating that the forces due to the substrate surface plays a less critical role compared to internanorod forces. At concentrations between 50 and $120 \mathrm{mM}$ (region 2 of Figure 7-2G), the first shielding effect at high CTAB concentration is notably less effective than at moderate $\mathrm{CTAB}$ concentration due to the presence of a large quantity of positively charged, free CTAB in the solution drawing the adsorbing anions away from the nanorod surface. This will result in a dilution of anions close to the GNR surface leading to a thicker double layer with better repulsive character. After the peak is reached (region 3 of Figure 7-2G), effects arising from CTAB molecules adsorbing on the substrate surface are observed to be negligible because excess CTAB molecules already starts to effectively participate in second electrostatic shielding in solution. Since we have no information on exactly how much excess CTAB participates in the second shielding effect, we cannot assume any possibility of excess CTAB adsorbing on the substrate surface anymore, especially when the prepared solution is left to stabilize prior to substrate immersion.

In order to confirm that nanorod-nanorod interactions is superior to nanorod-substrate interactions and rendering the substrate with surface positive charges does not significantly affect the surface assembly, a control experiment using APTS-functionalized substrates without negatively charged PSS layer was performed. Since APTS is positively charged, we expect immersing the substrate in AuNR 
solutions with increasing salt concentration would lead to higher density of deposited AuNRs. However, it is repeatedly found that zero surface assembly is observed under the same range of salt concentration. This confirmed, in support of $\zeta$-potential data, that the nanorods retained a net positive surface charge throughout the variation. While it is arguable that excess anions which have not participated in the first shielding may form a negatively charged layer over the APTS monolayer and therefore repel negatively shielded AuNRs, deposition should still occur at peak $\mathrm{NaCl}$ concentration when the amount of anions is only sufficient to form the shielding layer around the AuNRs. Finally, the fact that there is a direct correlation between $\zeta$ potential data and the absorbance trend strongly suggests that nanorod-nanorod interactions are primarily contributing.

SEM images of the nanorod surface assembly on silicon are shown in Figure 7-4 to visualize the density of the nanorods and to confirm that the intensity of absorbance corresponds to the density of the assembled nanorods. As shown in Figure 7-4B, a generally well-distributed surface assembly of AuNRs is observable at $40 \mathrm{mM} \mathrm{NaCl}$. In some regions, side-to-side and end-to-end coupling of nanorods can be seen. With respect to DLVO theory used earlier in the discussion, it can be suggested that at some points the van der Waals attraction may dominate over the double-layer repulsion between nanorods. When a nanorod is successfully deposited due to the electrostatic attraction originating from the surface, a redistribution of charges occur around that nanorod. Since the van der Waals forces are partially due to transient distribution of charges, this redistribution of charges can cause either an increase or a decrease of attractive van der Waals force on another nanorod within its proximity. If it increases sufficiently to dominate over the double-layer repulsion force of the second nanorod, it will be deposited adjacent to the first. As the inherent double-layer repulsion forces of 
AuNRs in solution become weaker at higher salt concentration, such occurrences also become more widespread. This is observed at a peak $\mathrm{NaCl}$ concentration of $80 \mathrm{mM}$ (Figure 7-4C) when the dense surface assembly is not only directly caused by the electrostatic forces of attraction of the surface but also due to the redistribution of charges around nanorods during instances of deposition. When double-layer repulsion forces are regained significantly from $\mathrm{NaCl}$ concentration of 120 to $160 \mathrm{mM}$, a reverse trend occurs.

Therefore, in the context of electrostatic surface assemblies, while it may be arguable to use high concentration of salt ${ }^{312}$, it may still be beneficial to introduce salt within an optimal range. In the previous sections, it was shown that this interdependently allows for the use of moderate concentrations of CTAB or any other stabilizing surfactants essential in preventing the deformation of AuNRs, in prolonging the shelf life of the solution, and in achieving surface assembly of AuNRs with good tunability. The overall trend and various phenomena explained are summarized and illustrated in a simplified schematic of Figure 7-5. 
1

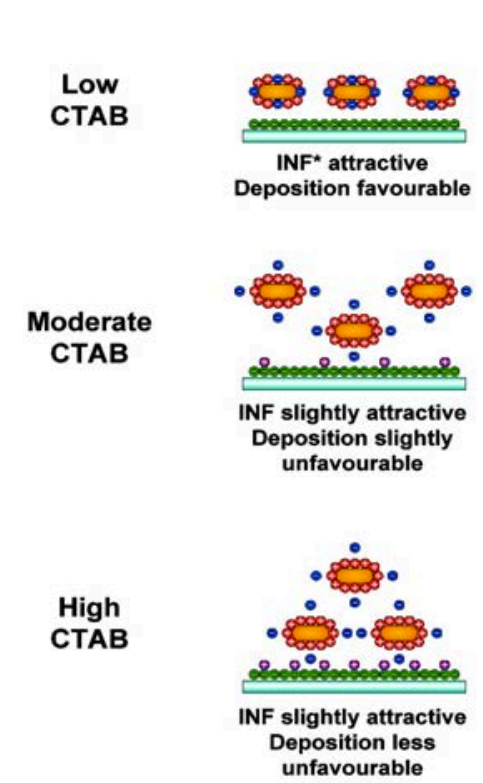

2
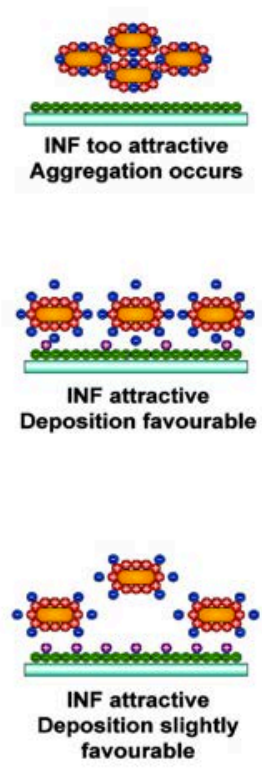

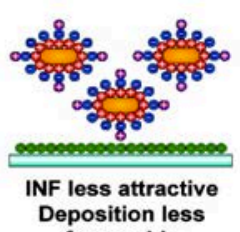

favourable

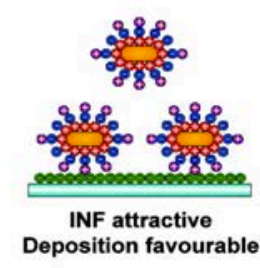

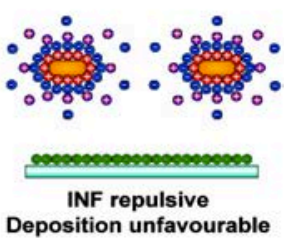

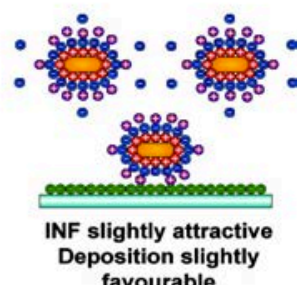

Increasing lonic Strength

* INF - Inter-Nanorod Forces

Figure 7-5. Tabulated simplified schematics showing the internanorod as well as nanorod-substrate interactions under increasing ionic strength at different $\mathrm{CTAB}$ concentrations. The numbers correspond to the ionic strength regions as referred to in the discussion and denoted in the plot of Figure 7-2. Here "attractive" is a relative term used to describe internanorod forces which are not sufficiently repulsive to prevent surfacial deposition; such "attractive" forces are however not necessarily strong enough to cause nanorod aggregation in solution. The relative sizes of the gold nanorod, CTAB bilayer, salt anion, and free CTAB molecules are not to scale. Reprinted with permission from Langmuir, 2010, 26 (14), pp 12433-12442. Copyright 2010 American Chemical Society.

\subsubsection{Robustness of electrostatic nanorod surface assembly}

Since substrate assembly of AuNRs, when used as a biomolecular detection platform, has high chances of exposure to a dynamic fluid environment both in in vitro and in vivo applications, it is critical to ensure that AuNRs do not desorb from the 
surface and get washed away easily. In addition, no significant peak shift caused by any possible aggregation should occur to ensure that any subsequent detectable peak shifts are solely due to biomolecular binding.

Here, we compared the nanorod retention properties of assemblies with similar initial peak absorbances obtained from the solvent evaporation method with that from the full immersion method from solution at low CTAB concentration. We also compared nanorod retention of assemblies obtained from solutions at moderate and high CTAB concentrations which produced highest absorbance (at $120 \mathrm{mM} \mathrm{NaCl}$ ). The distinct longitudinal peak of AuNRs prepared by solvent evaporation became clearly diminished after the first wash with PBS (Figure 7-6A). Instead, a broad shoulder is observed between wavelengths of 700-900 $\mathrm{nm}$. Here, the solvent used is not added with any salt to adjust its ionic strength, and the precleaned substrate surface is not further treated. Hence, the surface assembly is primarily contributed by capillary forces which resulted from the evaporation of water from the surface. This clearly suggests that without any modifications such as ionic strength and surfactant concentration the solvent evaporation method may not be appropriate to produce assemblies that withstand dynamic fluid environments. While the longitudinal absorbance peak disappeared, it was experimentally observed that the tint remained on the slip even after two rounds of washing. This indicates that peak disappearance is not mainly due to nanorod desorption from the surface but nanorod aggregation on the surface (Figure 7-7B). Such aggregation may be triggered by the counterions introduced in PBS. As there is an absence of a strong electrostatic force from the surface, the nanorods are relatively weakly bound to the hydroxylated surface. When immersed in a liquid, there is a high tendency for the nanorods to desorb. However, especially in the presence of counterions, most of the nanorods that are desorbing from 
the surface are attracted to neighboring nanorods possibly due to electrostatic shielding. As a result, they will collectively aggregate on the surface.

A

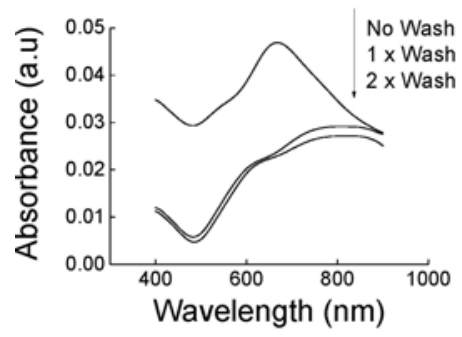

C

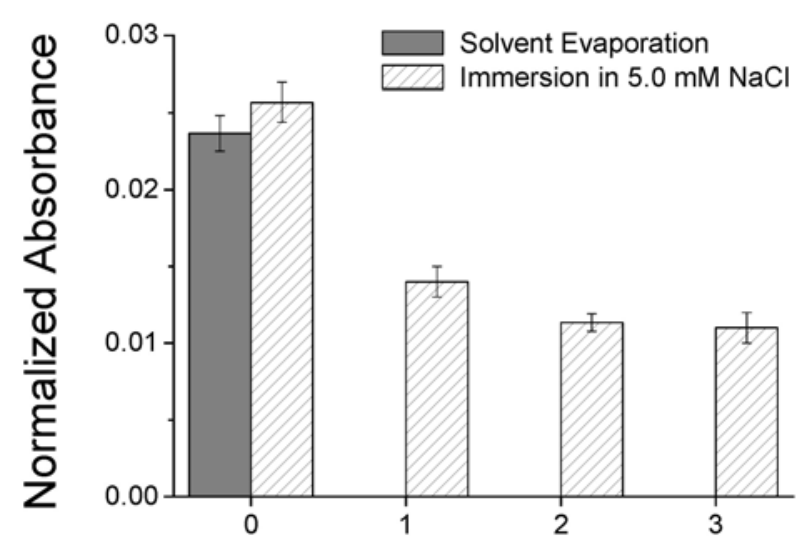

No. of Washing Steps

B

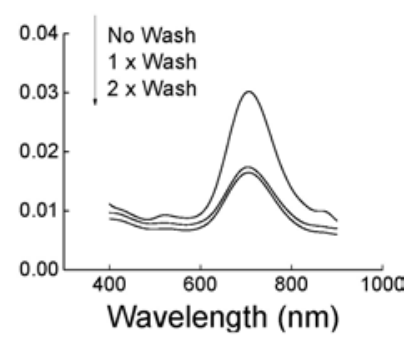

Solvent Evaporation

mmersion in $5.0 \mathrm{mM} \mathrm{NaCl}$

\section{3}

$$
\text { (1) }
$$

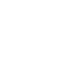 \\ (1)}

Figure 7-6. UV-vis absorbance spectra showing the decrease in absorbance for samples prepared via (A) solvent evaporation and (B) full immersion of glass in solution of low CTAB concentration at $5.0 \mathrm{mM} \mathrm{NaCl}$. (C) A graphical comparison of the decreasing trend of absorbance spectra. Error bars represent standard deviation. Reprinted with permission from Langmuir, 2010, 26 (14), pp 12433-12442. Copyright 2010 American Chemical Society. 


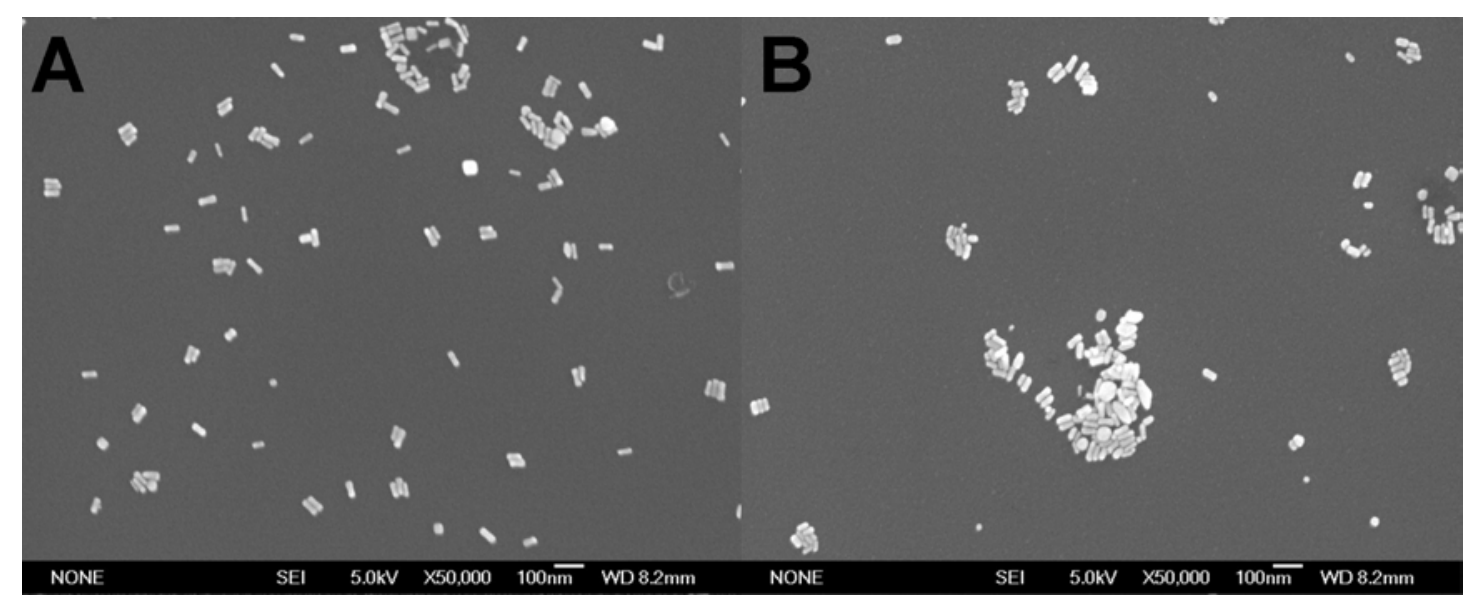

Figure 7-7. SEM images of gold nanorod surface assembly on silicon substrate obtained from the solvent evaporation method (A) before and (B) after washing. Reprinted with permission from Langmuir, 2010, 26 (14), pp 12433-12442. Copyright 2010 American Chemical Society.

On the other hand, it is shown in Figure 7-6B that the surface assembly based on the full immersion method effectively retained its longitudinal peak position and shape. After the first wash, the intensity of absorbance decreases. This may also be due to desorption of weakly bound nanorods. However, unlike the case of substrates by solvent evaporation, the amount of desorbed nanorods is less since more nanorods would be tightly bound to the surface. Subsequently, minimal absorbance drop is observed between the first and second washing steps, indicating that the surface is now coated with only nanorods which are tightly bound by the strong electrostatic force originating from the PSS layer. This trend is presented in Figure 7-6C. Clearly, the presence of an electrostatic surface charge is beneficial to produce assemblies with good nanorod retention.

To further study the retention characteristics obtained from the full immersion method, assemblies with high nanorod densities were utilized. These are assemblies obtained from solutions of moderate and high CTAB concentrations at $120 \mathrm{mM} \mathrm{NaCl}$. From Figures 7-8A \& $\mathbf{B}$, it is evident that the longitudinal peak is preserved albeit a 
drop in absorbance after the first and second washing steps and a slight blue-shifting occurring after the second wash. No further blue-shifting occurred after the third wash. We suspect that during initial assembly adjacent nanorods couple side-to-side due to high density of AuNRs on the substrate. After washing, a fraction of uncoupled nanorods desorb exposing coupled nanorods which remain fixed. When this occurs, absorption intensity decreases while the relative contribution toward the signal by the remaining coupled nanorods would be slightly higher, causing slight blue-shifting. Regions of exposed side-to-side coupled nanorods and clusters are observed under SEM and shown in Figure 7-9. Generally, coupled pairs of nanorods are sporadic but well-distributed over the surface. Clusters are, however, less frequently observed. Nonetheless, the image is included to emphasize that the existence of a few clusters may also explain for the slight blue-shift. Such blue-shifting after washing steps is obviously absent for lower density surface assembly (Figure 7-6B). However, it is unclear why blue-shifting occurs more drastically during the second washing step instead of the first. This was consistently observed with repeated experiments. The decreasing trend in absorbance depending on the number of washing steps is presented in Figure 7-8C. The total drop in mean absorbance after the third washing step is $39.5 \%$ and $36.0 \%$ for assemblies obtained from moderate and high CTAB concentration. While these values are comparable, the absorbance drop in the low density surface assembly at low CTAB concentration (57.1\%) is significantly higher than those in the high-density assemblies. Although, at this point, we cannot deduce whether this difference is due to the nature of the electrostatic attraction of the surface or the difference in nanorod density, we can conclude that repeated rigorous washing steps is necessary to obtain a stable and reliable nanorod surface assembly. 
A

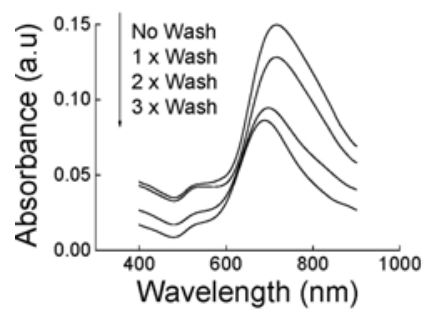

C
B

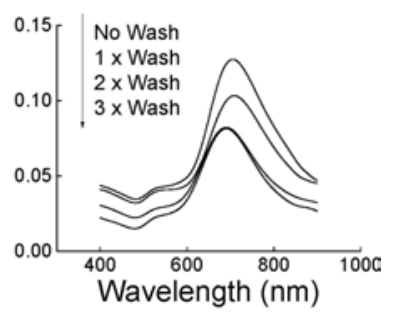

Moderate CTAB WII High CTAB

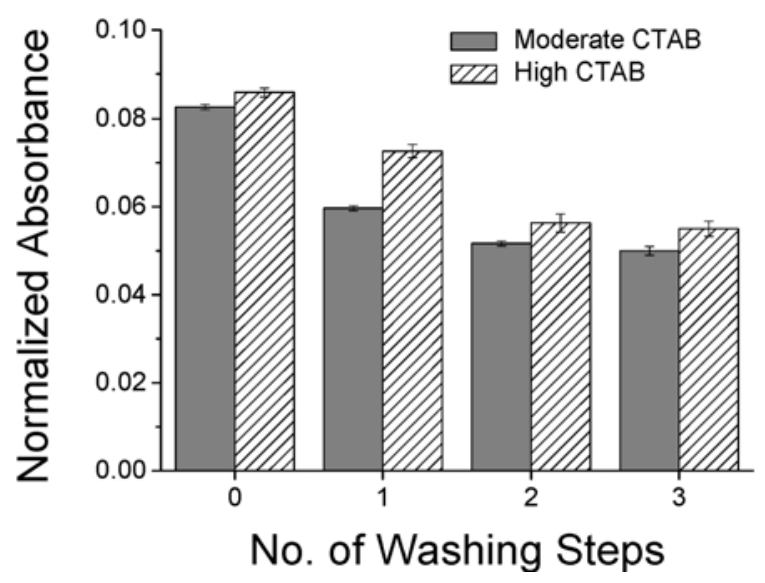

Figure 7-8. UV-vis absorbance spectra showing the decrease in absorbance for samples prepared via full immersion of glass in solution under (A) moderate CTAB concentration at $120 \mathrm{mM}$ and (B) high CTAB concentration at $120 \mathrm{mM} \mathrm{NaCl}$. (C) A graphical comparison of the decreasing trends. Error bars represent standard deviation. Reprinted with permission from Langmuir, 2010, 26 (14), pp 12433-12442. Copyright 2010 American Chemical Society.

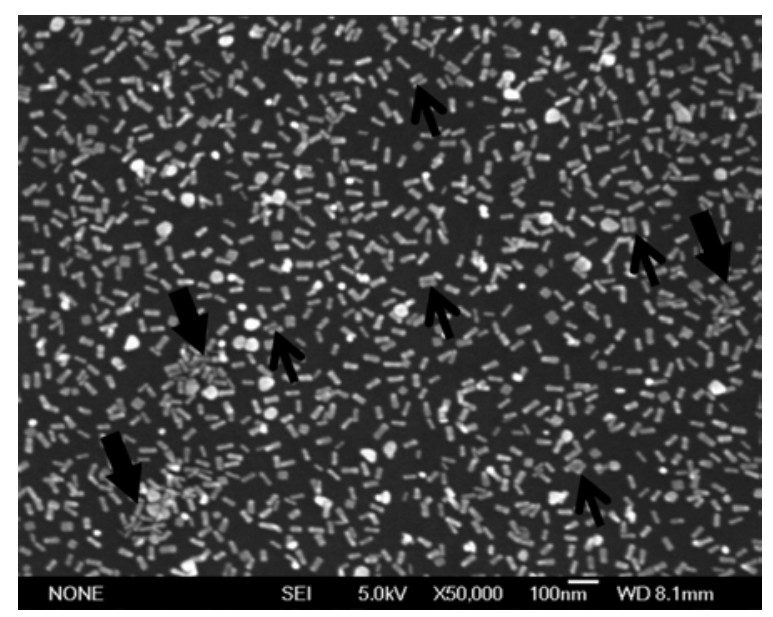

Figure 7-9. SEM images of gold nanorod surface assembly on silicon substrate from a solution of moderate $\mathrm{CTAB}$ concentration at $120 \mathrm{mM} \mathrm{NaCl}$ after second round of 
washing. Regions of side-to-side couplings and clusterings are indicated by the thin and thick arrows, respectively. Reprinted with permission from Langmuir, 2010, 26 (14), pp 12433-12442. Copyright 2010 American Chemical Society.

\subsection{Conclusion}

We presented herein the controllable surface assembly of AuNRs on fully immersed glass substrates by adjusting both the ionic strength and CTAB concentration of the AuNR solution. Glass substrates which were modified to yield a net negative charge were used. We conclude that the electrostatic surface assembly of AuNRs can be effectively mediated through the anions present in the AuNR solution. At a slightly excess CTAB concentration, a highly dense assembly is obtained under optimal ionic strength of around $100 \mathrm{mM} \mathrm{NaCl}$. A comprehensive, qualitative discussion explaining the various trends observed across different regions of ionic strength offers a better understanding on the different mechanisms involved in surface assemblies of surfactant-capped AuNRs on fully immersed substrates based upon electrostatic interactions. A detailed study on the kinetics of these mechanisms will be performed in future works. In a noteworthy addition, we studied the robustness of such electrostatic assemblies. Unlike assemblies obtained from a simple solvent evaporation method, the longitudinal peak from our full immersion method remained distinct even after three rounds of washing in PBS. It was also found that no further decrease in absorbance and peak-shifting occurred after the second round of washing. This concludes that electrostatic assemblies via the full immersion method are reliable to be used in biosensing platforms equipped with microfluidics as well as in implantable devices. 


\section{Chapter 8}

\section{Gold Nanorod Adsorption on POEGMA and Its Application in Surface Enhanced Raman Spectroscopy}

\subsection{Introduction}

Due to their inherent ability to scatter and absorb light as a consequence of localized surface plasmon resonance (LSPR), noble metal nanoparticles (NMNPs) have received substantial interest in various fields including, but not limited to, photonics $^{107,109}$, optoelectronics ${ }^{108,324}$, and biomedical diagnostics ${ }^{325-327}$. Among the plethora of NMNPs, gold nanorods (AuNRs) serve as the most basic yet ever-relevant exemplification of how anisotropy can vastly widen the application scope of nanoparticles $^{116,117}$. The ability to conveniently vary the 'aspect ratio' (i.e. the ratio between the length and width of AuNRs) during synthesis gives rise to a tunable longitudinal LSPR extinction peak in addition to a relatively fixed transverse peak ${ }^{118}$. This allows AuNRs to exhibit light scattering and absorbance over a wider wavelength range otherwise not achievable by their spherical counterparts. This, for example, has extended the application of AuNRs towards in vivo biosensing ${ }^{328-330}$, bioimaging $^{331,332}$ and photothermal therapy ${ }^{163,333-335}$ since the longitudinal peak can be conveniently positioned within the 'biological transparency windows"120,121 (i.e. wavelength regions where biological tissues absorb and scatter less light, rendering lesser interference).

More interestingly, the two-dimensionality of the structure offers many different ways of adjoining two or more AuNRs, resulting in a variety of combined geometries. Since plasmon oscillations on adjacent nanoparticles can couple ${ }^{110}$, this would result in countless new plasmonic modes and associated LSPR peaks, especially when the 
AuNRs are in contact at non-quadrantal angles. While the plasmonic modes of end-toend, side-to-side and end-to-side coupled AuNRs existing as isolated dimers, trimers and multimers have been extensively studied ${ }^{336-338}$, the plasmonic modes of closepacked long-range assemblies, where continuous coupling occurs over several microns, have yet to be fully explored. Further investigation into such AuNR superstructures would accelerate the development of new materials for applications like surface-enhanced Raman spectroscopy (SERS). Since it has been established that anisotropic metal nanoparticles contribute to better SERS enhancements ${ }^{339}$, the assembly of AuNRs into superstructures would tremendously improve the SERS sensing performance due to the abundant number of nanoparticle junctions serving as electromagnetic 'hot-spots'.

Nonetheless, in order for such investigation to proceed, there first need to be convenient methodologies to facilitate the assembly of AuNR into uniform and homogeneous superstructures. While it is relatively easier to obtain close-packed assemblies of spherical gold nanoparticles on a substrate ${ }^{340}$, it is more challenging for the case of AuNRs mainly due to the existence of a surrounding layer of surfactant ${ }^{166,}$ ${ }^{341}$. In general, surfactants such as cetyltrimethylammoniumbromide (CTAB), needed for the anisotropic growth of AuNRs, remain tightly bound to the AuNR surface after synthesis, giving rise to strong inter-nanorod repulsive forces. As a consequence, asprepared AuNR solutions typically resist assembly on a substrate.

To overcome this, several groups have reported high density or even configuration controllable self-assemblies by removing or replacing the surfactant ${ }^{342,} 343$. Alternatively, the assembly could be obtained at the air/water interface before transferring to the substrate ${ }^{344}$. Without any modification to the AuNR or the surface, one popular approach to obtaining high-density assemblies is through the droplet 
evaporation method whereby the AuNR solution is simply dropped to the surface and allowed to dry ${ }^{345-347}$. While these methods may yield high-density assemblies over a short range, the assembly is often difficult to attain over a large area and requires precise control over the parameters governing the assembly process. At present, the implementation of such structures for practical applications is far-fetched due to the limited availability of fabrication processes that allow large size assemblies to be obtained with excellent lateral uniformity and homogeneous thickness. While such assemblies are excellent as proof-of-concept, they are not suitable for real-life applications.

In order to obtain assemblies suitable for device fabrication and consumer use, a much more straightforward, efficient, green and cost-effective approach is urgently needed. In our previous work, we have easily obtained AuNR assemblies with tunable density via a full immersion approach ${ }^{250}$. However, even at its highest density, many large gaps were still visible between neighbouring AuNRs and it was not possible to obtain three-dimensional assemblies. While obtaining high-density two-dimensional assemblies of metallic nanoparticles is now relatively common, obtaining high-density three-dimensional assemblies remains a challenge. At present, most works rely on additional microstructural scaffolds to be introduced onto the substrate to contribute to the vertical component of three-dimensional assemblies. For example, they could be obtained by assembling the nanoparticles on polymer scaffolds deposited on the substrate $^{348,349}$, on arrays of silicon nanowires (SiNW) grown from the substrate ${ }^{350}$ or even on a photolithographic microstructure ${ }^{351}$. The three-dimensionality of such platforms effectively arises from the scaffolds, not the nanoparticles, being arranged in a three-dimensional fashion. In other words, the nanoscale arrangement of the 
nanoparticles on the polymer, SiNW surface and photolithographic microstructure essentially still remains two-dimensional.

In this work, we introduce an extremely convenient approach to obtain closepacked, high-density two- to three-dimensional AuNR assemblies with macroscale lateral uniformity and homogeneous thickness. As the assemblies were obtained with SERS application in mind, we mimicked the formation of free-floating AuNR aggregates in solution, which have been traditionally known for many years to give tremendous SERS enhancements ${ }^{352,353}$. However, the oxymoronic challenge is to obtain uniform 'aggregates' and ensure that they are totally contiguous over the substrate surface. To achieve this, the AuNRs were assembled on poly(oligo ethylene glycol methacrylate) (POEGMA) brush-grown substrates by fully immersing the latter in as-prepared AuNR solution. Apart from possessing non-fouling properties, which could help eliminate non-specific adsorption of biomolecules ${ }^{227}$, POEGMA was chosen because we have previously shown that spherical gold nanoparticles were able to spontaneously bind to the brush without any functionalization ${ }^{218,354}$.

Specific to this case, the use of POEGMA has two more advantages. Firstly, it can be grown up to a thickness of around $100 \mathrm{~nm}$, enabling the accommodation of $100 \mathrm{~nm}$ long AuNRs in a variety of configurations including horizontal deposition onto the brush surface as well as tilted and vertical embedment into the depth of the brush. This consequently facilitates close packing, leading to a true type of three-dimensional assembly and ultimately plenty of SERS hot spots between adjacent AuNRs. Secondly, polymer brushes are able to swell in solution. This would enable the AuNRs to space out when the substrate is immersed in solution, giving target molecules access to a higher surface area of AuNRs, leading to increased adsorption. Subsequently when dried, the AuNRs would collapse upon each other naturally trapping the 
adsorbed molecules within the hot spots. The resulting AuNR assembly therefore resembles a 'floating super-aggregate', which, as far as we know, is the first representation of a dynamic unfunctionalized long-range superstructure attached to a substrate.

\subsection{Specific Methods}

\subsubsection{Gold nanorod self-assembly into floating super-aggregates}

The polymer brush-grown substrates were fully immersed in the AuNR solution for a specified duration ranging from 1 to $24 \mathrm{~h}$. For silicon substrates, they were placed in the wells of a 24 -well plate and immersed in $500 \mu \mathrm{L}$ of AuNR solution. For glass coverslips, they were placed in a standard $35 \mathrm{~mm}$ disposable petri dish and immersed in $3 \mathrm{~mL}$ of AuNR solution. No shaking or agitation was needed. They were then removed and rinsed copiously with DI $\mathrm{H}_{2} \mathrm{O}$ and blown dry with $\mathrm{N}_{2}$.

\subsubsection{Characterization}

The brush thickness was measured from silicon substrates by ellipsometry in air using a J.A. Woolam $\alpha$-SE spectroscopic ellipsometer. UV-vis spectra were obtained from the glass coverslips using a Shimadzu UV-2540 UV spectrophotometer. AFM data from the silicon substrates was obtained in air using the Asylum Research MFP3D AFM system conducted in tapping mode. Scanning electron micrographs were obtained from both the silicon substrates as well as the glass coverslips using a JEOL JSM-6700F FESEM microscope.

\subsubsection{SERS measurements}

SERS signals were obtained using Rhodamine 6G (R6G) as the probe molecule. Silicon substrates with the AuNR floating super-aggregates were immersed in $500 \mu \mathrm{L}$ 
aqueous solution of R6G with different concentrations ranging from $10^{-3}$ to $10^{-18} \mathrm{M}$ for $6 \mathrm{~h}$, rinsed thoroughly in DI $\mathrm{H}_{2} \mathrm{O}$ and blown dry with $\mathrm{N}_{2}$. All SERS spectra were collected using a Renishaw inVia Reflex Raman spectrometer mounted on a Leica microscope with a $50 \times$ objective lens $(\mathrm{NA}=0.80)$ in the range of $100-2000 \mathrm{~cm}^{-1}$ with one accumulation and $10 \mathrm{~s}$ exposure time. A $633 \mathrm{~nm}$ wavelength laser $(85 \mu \mathrm{W}$ at the sample surface) was primarily used to excite the sample; 514 and $785 \mathrm{~nm}$ laser was also used during optimization.

\subsection{Results and Discussion}

\subsubsection{Significance of ionic strength adjustment for nanorod adsorption on POEGMA}

We have previously shown that the adjustment of ionic strength can greatly facilitate AuNR deposition on a substrate. Based on the same principle, we adjusted the ionic strength of the AuNR solution after synthesis prior to deposition. Only a slight blue-shift in the longitudinal absorbance peak was observed from the UV-vis spectra (Figure 8-1), with no aggregation upon standing. Substrates grown with poly(oligo ethylene glycol methacrylate) (POEGMA) polymer brush up to a thickness of $100 \mathrm{~nm}$, as measured via ellipsometry, were then immersed in the AuNR solution (Figure 8-2). As a comparison, we also performed the immersion of bare substrate in ionic strength-adjusted AuNR solution, as well as POEGMA-treated substrate in nonadjusted AuNR solution. None of the other two combinations resulted in any significant deposition (Figure 8-3), implying that both ionic strength adjustment and polymer brush was needed for successful AuNR assembly. We further varied the $\mathrm{NaCl}$ concentration and observed a parabolic trend between the amount of AuNR deposition and $\mathrm{NaCl}$ concentration, with $0.05 \mathrm{M} \mathrm{NaCl}$ giving the highest amount of AuNR 
deposition (Figure 8-4). This was analogous to the trend found in our previous work, implying that the assembly in this work also arises from electrostatic interactions. However, it is interesting to note that unlike the work in Chapter 7, AuNR assemblies on POEGMA yielded UV-vis spectra with negative absorbance values in some wavelength regions. Given the reference sample being POEGMA on glass without AuNR, negative absorbance from measured samples can only be physically interpreted as an increase in transmittance of incident light (i.e. at certain wavelengths, more light is transmitted through AuNR deposited POEGMA compared to bare POEGMA). While the mechanism for this remains unclear, there exist several works in literature attributing increase in transmittance to extraordinary optical transmission (EOT) ${ }^{355-357}$ as well as plasmonic Fano resonance. ${ }^{358-360}$ An in-depth investigation involving both experiments and simulations may have to be performed separately in order to unravel the contributing factor to this unusual phenomenon for this particular case of AuNR assembly on POEGMA.

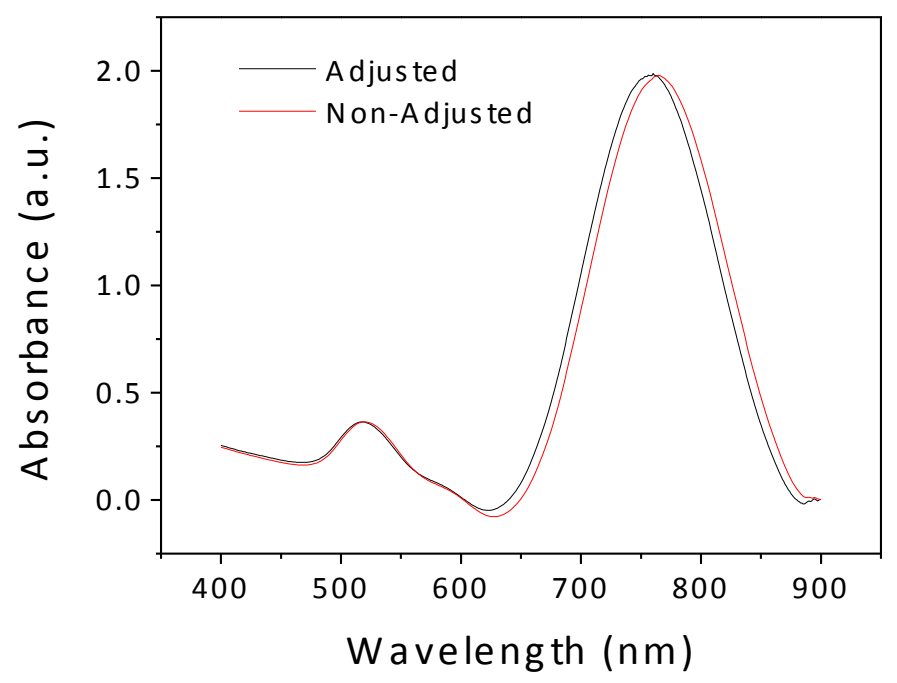

Figure 8-1. UV-vis spectra obtained from as-prepared AuNR solution with and without ionic strength adjustment $(0.05 \mathrm{M} \mathrm{NaCl})$. 


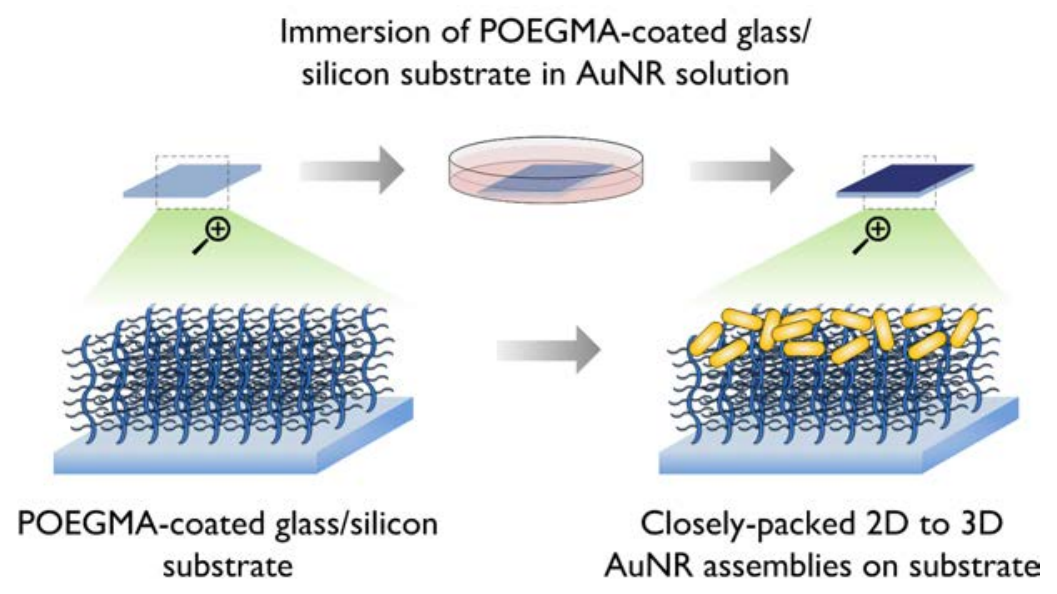

Figure 8-2. Simplified representation of the one-step full immersion procedure to obtain close-packed two- to three-dimensional AuNR assemblies.

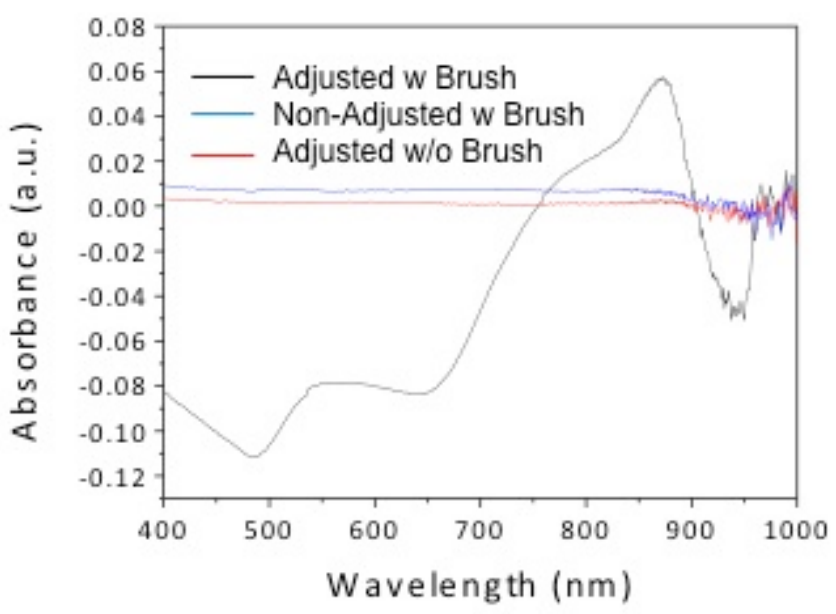

Figure 8-3. UV-vis spectra obtained from POEGMA-grown glass substrates immersed overnight in AuNR solution with (black trace) and without (blue trace) $0.05 \mathrm{M} \mathrm{NaCl}$ ionic strength adjustment. UV-vis spectra obtained from bare glass substrates immersed overnight in AuNR solution with $0.05 \mathrm{M} \mathrm{NaCl}$ ionic strength adjustment (red trace). 

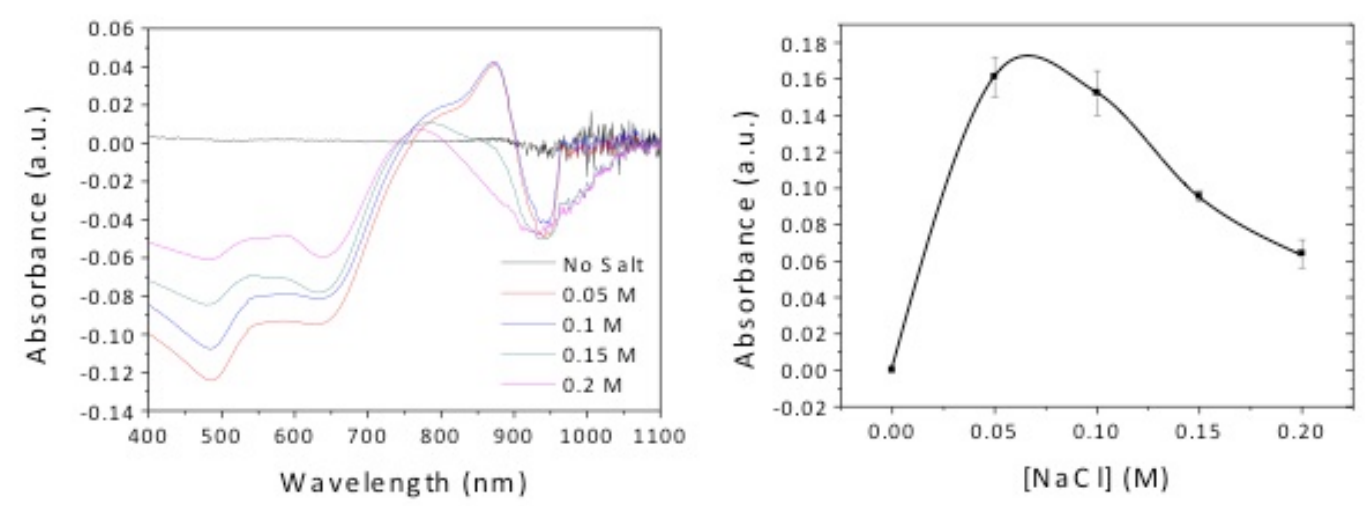

Figure 8-4. Left: UV-vis spectra obtained from POEGMA-grown glass substrates immersed overnight in AuNR solution adjusted with different $\mathrm{NaCl}$ concentrations. Right: Plot of integrated area under the UV-vis spectra versus $\mathrm{NaCl}$ concentration.

\subsubsection{Effect of brush thickness on gold nanorod assembly}

By varying the immersion time from $1 \mathrm{~h}$ to $24 \mathrm{~h}$, we observed the gradual evolution of the UV-vis-NIR spectra from the deposited AuNRs (Figure 8-5A). For 1 $\mathrm{h}$ immersion, the common transverse and longitudinal peaks were observed at $530 \mathrm{~nm}$ and $760 \mathrm{~nm}$, respectively. As immersion time is increased to $3 \mathrm{~h}$, we observed a minimal red-shift in the transverse peak but a significant red-shift in the longitudinal peak from $760 \mathrm{~nm}$ to $775 \mathrm{~nm}$. In addition, a shoulder began to appear in the nearinfrared (NIR) region at around $870 \mathrm{~nm}$. Interestingly, at $6 \mathrm{~h}$ this shoulder developed into a distinct peak while the longitudinal peak downgraded to a shoulder. This NIR peak, which then remained fixed at $875 \mathrm{~nm}$, became sharper while the longitudinal shoulder became more diminished as the immersion time was further increased from 6 to 12 and $24 \mathrm{~h}$. We believe that this new peak is contributed by long-range plasmonic coupling, which occurred due to the AuNRs forming a contiguous assembly on the POEGMA. So far, we have yet to find any other report of such observation arising from AuNR deposition on a substrate. This is probably due to the difficulty in 
obtaining closely packed AuNR assemblies over areas large enough for such longrange coupling to occur and be observable using a common UV-vis spectrophotometer. For example, most drop-and-dry methods only give closely packed AuNRs in the "coffee stain" region, making it difficult to detect any long-range plasmonic coupling. In this work, however, we could see even with bare eyes that the AuNR deposition with $24 \mathrm{~h}$ immersion time is dense and uniform throughout the entire surface of the glass coverslip (Figure 8-5B). The same was observed for silicon substrates, which clearly showed a golden tinge after AuNR deposition. Interestingly, a deep purplish tint was observed when wetted, a phenomenon obviously not observable in gold-coated substrates (Figure 8-5C). This provided an initial hint that the AuNRs can be re-arranged when substrate is wet.
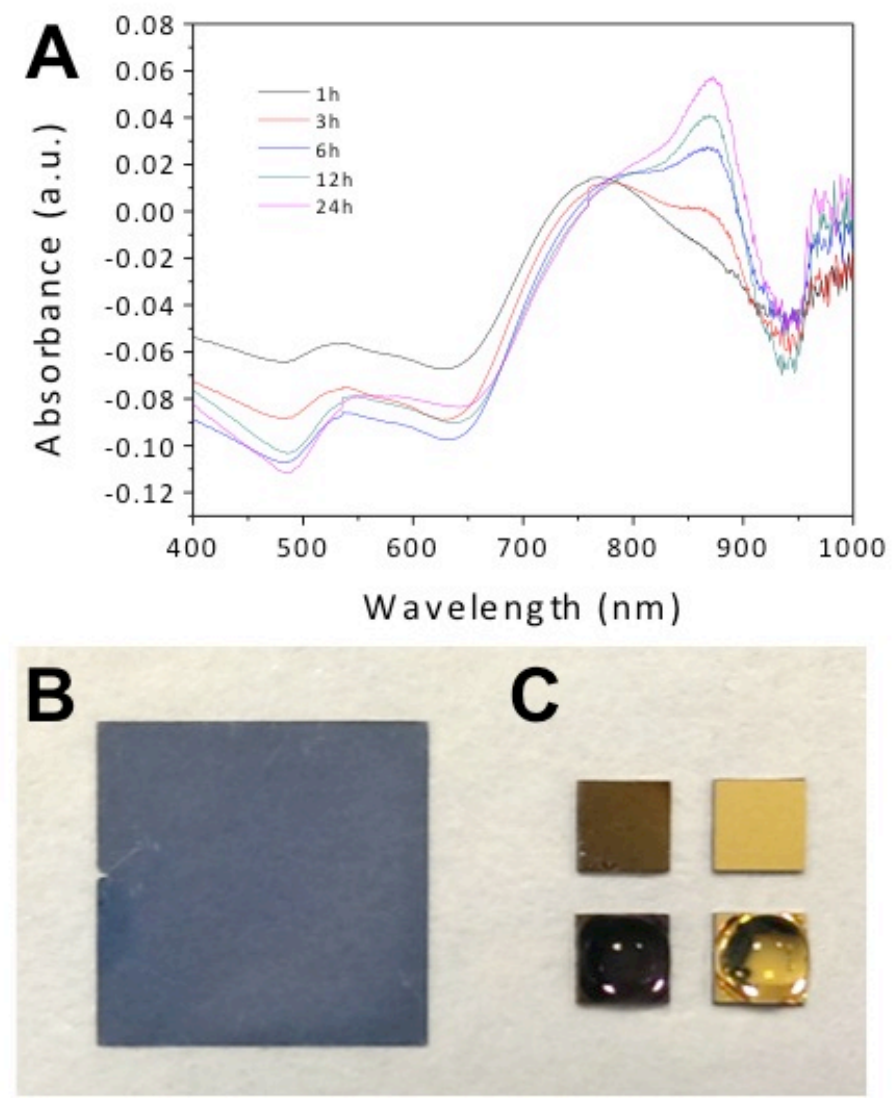

Figure 8-5. (A) UV-vis-NIR spectra obtained from POEGMA-grown glass coverslips after immersion in AuNR solution for 1, 3, 6, 12 and 24 h. (B) Photograph of 
POEGMA-grown glass coverslip after immersion in AuNR solution for $24 \mathrm{~h}$. (C) Photographs of POEGMA-grown silicon substrates after immersion in AuNR solution for $24 \mathrm{~h}$, in dry (top left) and wet (bottom left) states. Conventional gold-coated silicon substrates in dry (top right) and wet (bottom right) states are included for comparison.

To confirm the gradual formation of a contiguous AuNR assembly, we visualized the subtrates via SEM. We found that at $1 \mathrm{~h}$, the distribution is sparse with most of the AuNRs deposited in isolation (Figure 8-6A). This allows both the transverse and longitudinal peak to be clearly resolved. At $3 \mathrm{~h}$, a fraction of the AuNRs is deposited in a coupled state (Figure 8-6B). However, the distance over which coupling occurred did not extend more than one micron with coupled AuNRs mainly existing in small clusters. This explains the formation of a shoulder at the NIR region while the longitudinal peak red-shifted. At $6 \mathrm{~h}$, almost all the AuNRs are coupled leading to the sharpening of the NIR peak (Figure 8-6C). However, there are still some areas unoccupied by the AuNRs. At $12 \mathrm{~h}$ and above, the AuNRs completely cover the surface except for the ridges forming what appeared like a continous film of AuNR "super-aggregate" (Figure 8-6D). This leads to further sharpening of the NIR peak. 

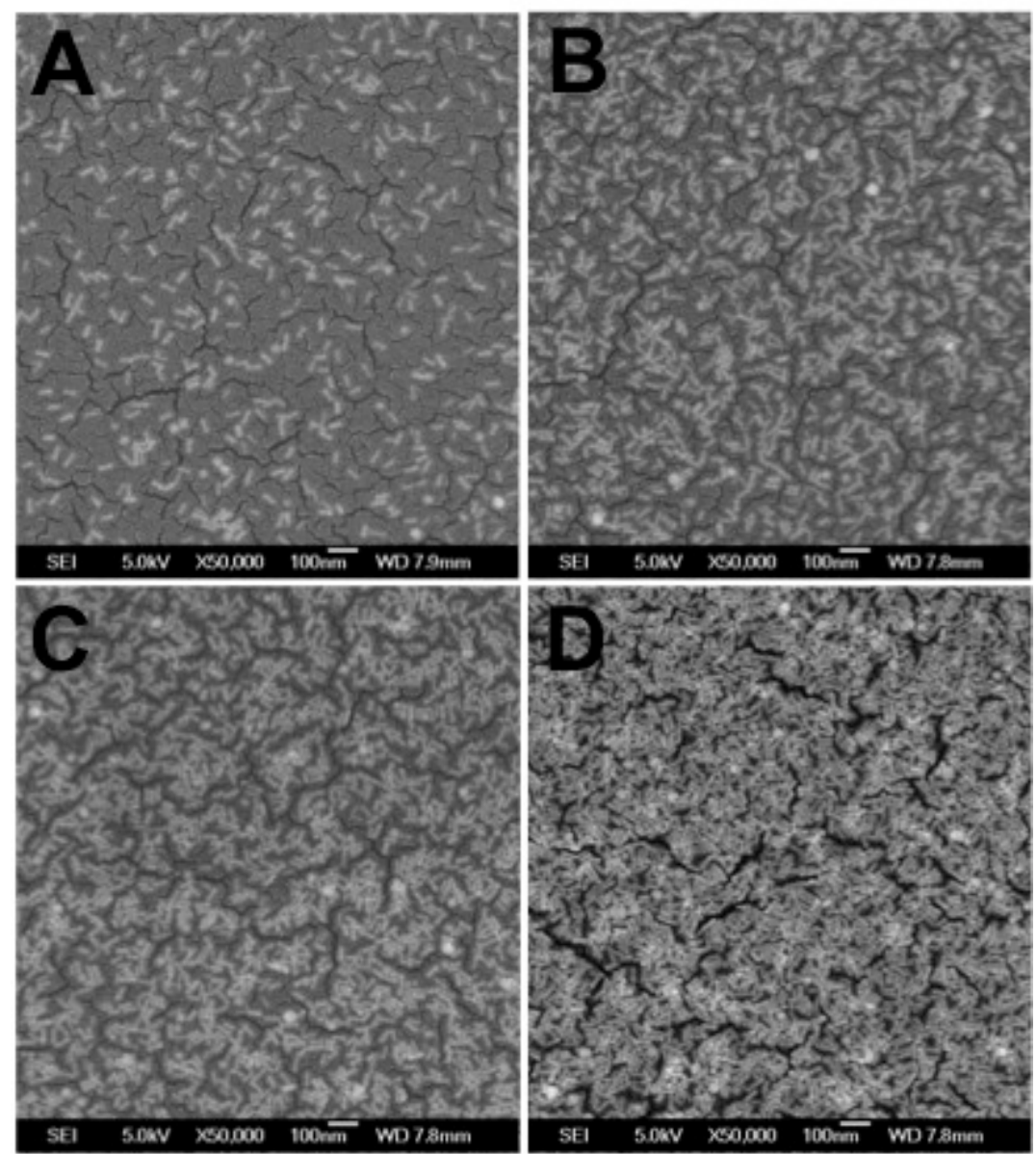

Figure 8-6. SEM images obtained from POEGMA-grown substrates after immersion in AuNR solution for (A) $1 \mathrm{~h}$, (B) $3 \mathrm{~h}$, (C) $6 \mathrm{~h}$ and (D) $24 \mathrm{~h}$.

We then proceeded to check the AFM images to observe more clearly the arrangement of AuNRs within the polymer brush volume. AFM height and phase retraces of bare POEGMA-grown substrate are included as comparison (Figure 8-7A). We found that AuNRs assembled with $1 \mathrm{~h}$ immersion time (Figure 8-7B) are hardly observed in the height retrace, although clearly observable in the phase retrace. In fact, in the height retrace, AuNRs are manifested as darker regions instead of brighter regions. In other words, they are slightly depressed at the top region of the brush. Even with $24 \mathrm{~h}$ immersion time, only some regions clearly show up as bright spots in the height retrace while the dense assembly of AuNRs is clearly observable in the phase retrace (Figure 8-7C). This indicates that most AuNRs are assembled at the sub- 
surface of the brush. This is interesting since it implies that the deposition of AuNRs does not sandwich the brush between the AuNR and the substrate surface, which would hamper brush chains from any movement. Instead, the AuNRs are floating on the brush and the swelling capability of the brush is somewhat retained.

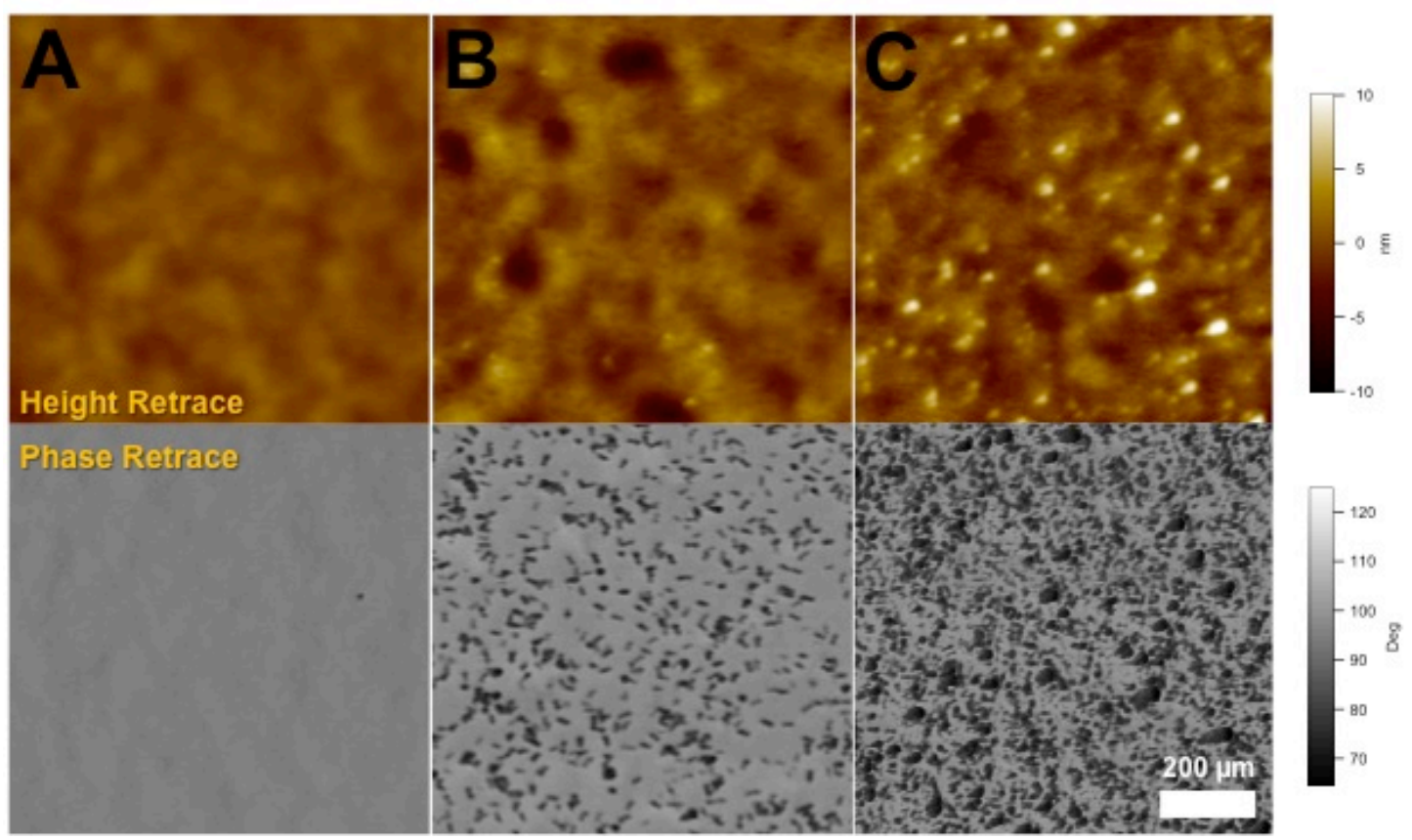

Figure 8-7. AFM height and phase retraces obtained from (A) bare POEGMA-grown silicon substrate and POEGMA-grown silicon substrates after immersion in AuNR solution for (B) $1 \mathrm{~h}$ and (C) $24 \mathrm{~h}$.

In order to check this, we compared the UV-vis-NIR spectra of the AuNR assemblies with different packing densities in dry and wet states. These experiments were done almost immediately after the final washing step. The 'dry spectra' were obtained after the samples were blown dry, while the 'wet spectra' were obtained when the samples were being re-immersed in water thereafter. We observed that for 3 $\mathrm{h}$, where packing density is low and NIR peak is absent, the longitudinal peak is blueshifted from dry to wet and the peak became slightly narrower (Figure 8-8A). We also 
found through subsequent brief wet-dry repetitions (3-4 times) that these variations were relatively reversible, although the reversibility became less obvious after each subsequent repetition. For 6 h, the sharp NIR peak clearly reduced to a shoulder when in wet state leading to the re-attainment of a clear longitudinal peak (Figure 8-8B). This served as evidence that the AuNRs undergo some degree of re-arrangement when placed in solution, which lead to the de-coupling of most of the clusters. As with $3 \mathrm{~h}$, the reversibility for $6 \mathrm{~h}$ also became impaired after each subsequent repetition. In the case of $12 \mathrm{~h}$, where the AuNR density is very high, the reduction in the NIR peak absorbance is still observable although the re-attainment of the longitudinal peak did not occur (Figure 8-8C). This suggests that re-arrangement occurs to a lesser degree as the AuNRs have lesser space to mobilize. Subsequent wet-dry repetition also did not yield satisfactory reversibility; clearly less than 3 and $6 \mathrm{~h}$. Nonetheless, all these evidences interestingly point towards the AuNRs having the ability to move and rearrange, particularly when freshly prepared. Such dynamic nature is especially crucial for high-density assemblies used in SERS applications, since it would still allow the probe molecules good access to the AuNR surface. For real-life practical applications however, it would perhaps be necessary to store the samples in wet environment prior to use, to ensure that the AuNRs retain optimal ability to be mobilized. 

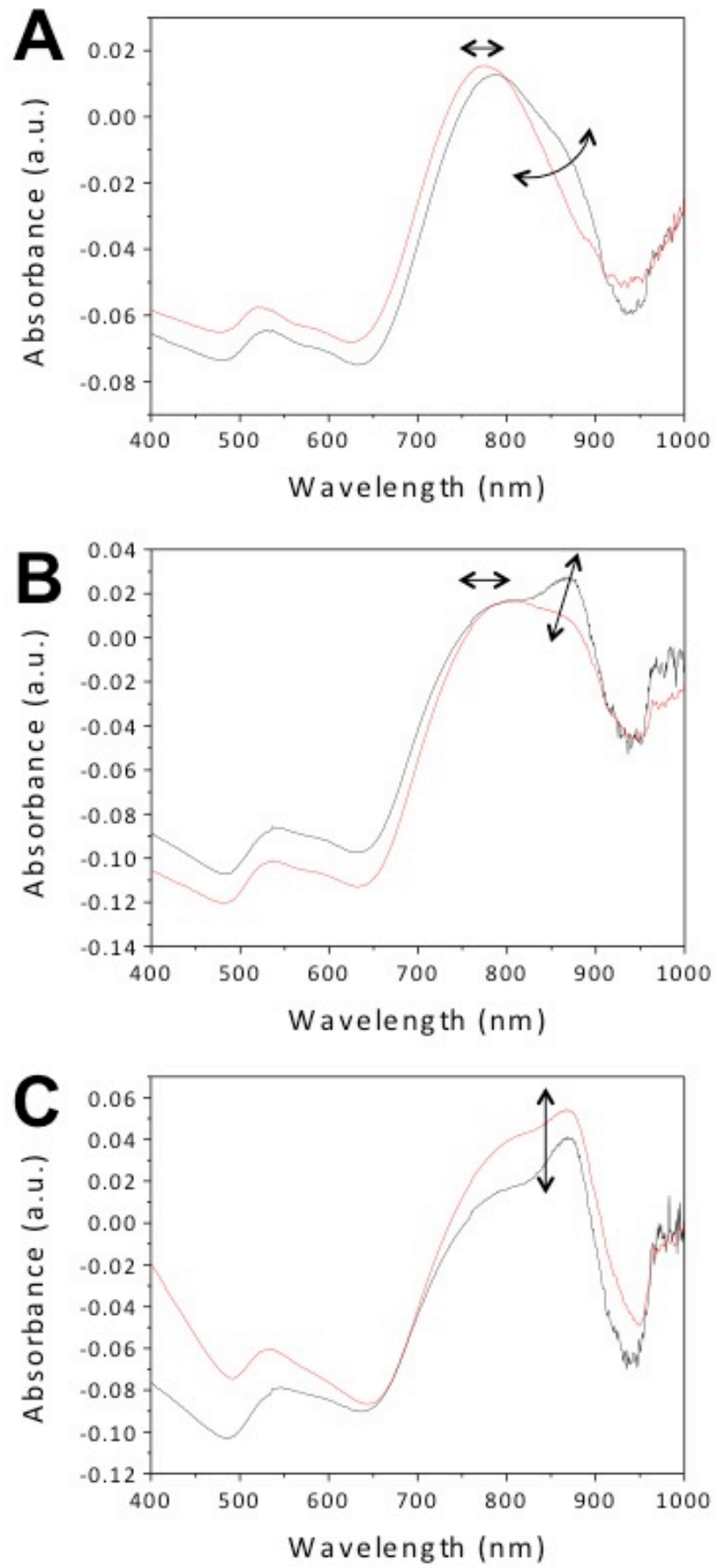

Figure 8-8. UV-vis-NIR spectra obtained from POEGMA-grown glass coverslips immersed in AuNR solution for (A) $3 \mathrm{~h}$, (B) $6 \mathrm{~h}$ and (C) $12 \mathrm{~h}$, taken in dry (black trace) and wet (red trace) states. 


\subsubsection{Uniformity of gold nanorod assembly over large areas}

One long-standing obstacle with regard to obtaining closely packed AuNR assemblies is the fact that it is difficult to extend over large areas (i.e. micron range and above $)^{361}$. It is therefore important in our case to prove that while our assembly method is straightforward and does not involve any form of chemical modification or physical agitation like stirring or microfluidic flow, it is nonetheless able to effectively assemble AuNRs uniformly over large areas without regions of uncontrolled aggregation or clustering. We proceeded to check the uniformity of the assembly over larger areas by gradually scanning our samples at decreasing magnifications. From Figure 8-9A-F, we found that the assembly of AuNRs into two-dimensional superaggregates is uniform well over a hundred micron, with no signs of large random aggregates or clusters, making the platform potentially suitable for practical SERS applications. We believe that such uniformity is due to 1) the stability of the AuNR solution even after ionic strength adjustment, which ensures no premature aggregation before deposition as well as 2) the volumetric exclusion effect of the brush, which ensures that AuNRs are deposited one at a time, with each deposition only occurring in the vicinity of a preceding deposited AuNR. Consequently, this implies that formation of super-aggregates initially extends from small clusters, as was earlier observed in Figure 8-6B. This also explains the quick development of the NIR peak observed in Figure 8-5A. 


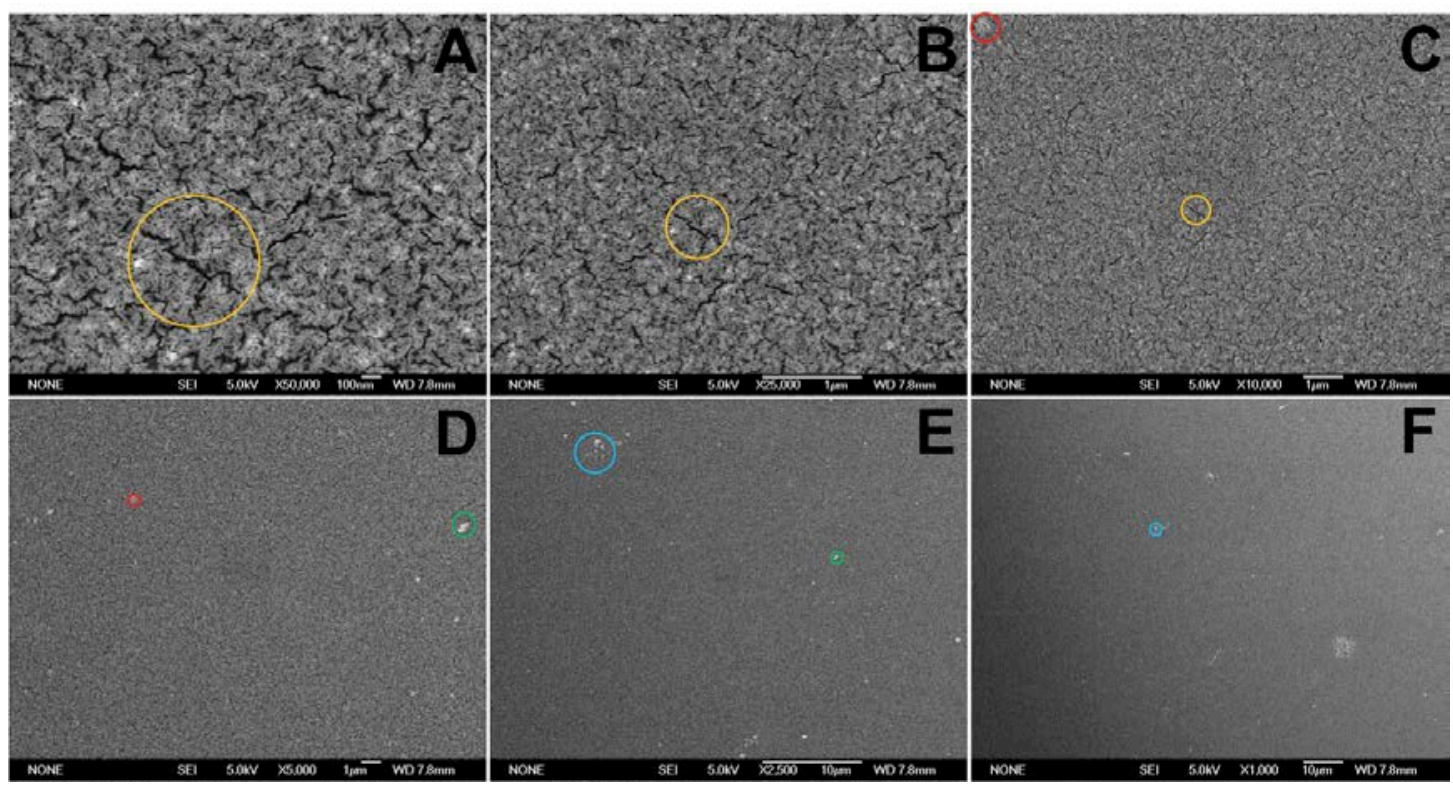

Figure 8-9. SEM images obtained from POEGMA-grown substrates after immersion in AuNR solution for $24 \mathrm{~h}$ taken at decreasing magnifications (i.e. zooming out) from (A) $50 \mathrm{k}$, (B) $25 \mathrm{k}$, (C) $10 \mathrm{k}$, (D) $5 \mathrm{k}$, (E) $2.5 \mathrm{k}$ and (F) $1 \mathrm{k}$; to guide the eye, colored circles are included to highlight common regions in images at different magnifications.

\subsubsection{Three-dimensional assembly of gold nanorods in POEGMA}

We further increased the packing density of the AuNR super-aggregate in order to transition from two- to three-dimensional assembly. This would increase the number of contact points between adjacent AuNRs and consequently the amount of SERS hotspots at the brush-solution interface. To achieve this, the AuNR concentration was increased two-, three-, five- and ten-fold with the immersion time fixed at $24 \mathrm{~h}$. Interestingly, we observed from the UV-vis-NIR spectra (Figure 8-10A) that doubling the concentration would cause the transverse peak and the longitudinal shoulder to converge into a smooth and almost linear trace of increasing absorbance intensity in the region of 500 to $800 \mathrm{~nm}$, culminating with a sharp NIR peak at $875 \mathrm{~nm}$. The disappearance of the transverse peak served as an early indication that a significant degree of AuNR stacking occurred, preventing individual transverse plasmon 
oscillations to be resolved. Instead, various coupling modes arising from AuNRs contacting at various angles average up to give an increasing overall absorbance intensity between 500 to $800 \mathrm{~nm}$. This is confirmed via SEM (Figure 8-10B), which showed higher density of closely packed AuNRs. As we further increased the concentration by three to five times, the overall absorbance increased, particularly with NIR peak becoming more distinct while remaining fixed at the same wavelength of $875 \mathrm{~nm}$. Surprisingly, when AuNR concentration was increased by ten times, the absorbance intensity decreased and the transverse peak re-appeared. From the SEM images we could see that the assembly became even denser with three and five times more concentrated AuNR, and the brush ridges became less apparent (Figures 8-10CD). However, with ten times more concentrated AuNR, the assembly became less dense, and the ridges became exposed (Figures 8-10E) even though the AuNR distribution seemed to remain relatively uniform. We proceeded to check SEM images at lower magnification (Figure 8-11) and found large random aggregates for the case of substrates immmersed in ten times concentrated AuNR. We concluded that when the concentration of the AuNR is too high, electrostatic destabilization occurs leading to some degree of aggregation in the AuNR solution even before deposition could take place. This effectively reduced the number of free AuNRs for assembly, leading to reduced packing density of uniformly distributed AuNRs and lower absorbance, allowing the transverse peak to be resolved again. 

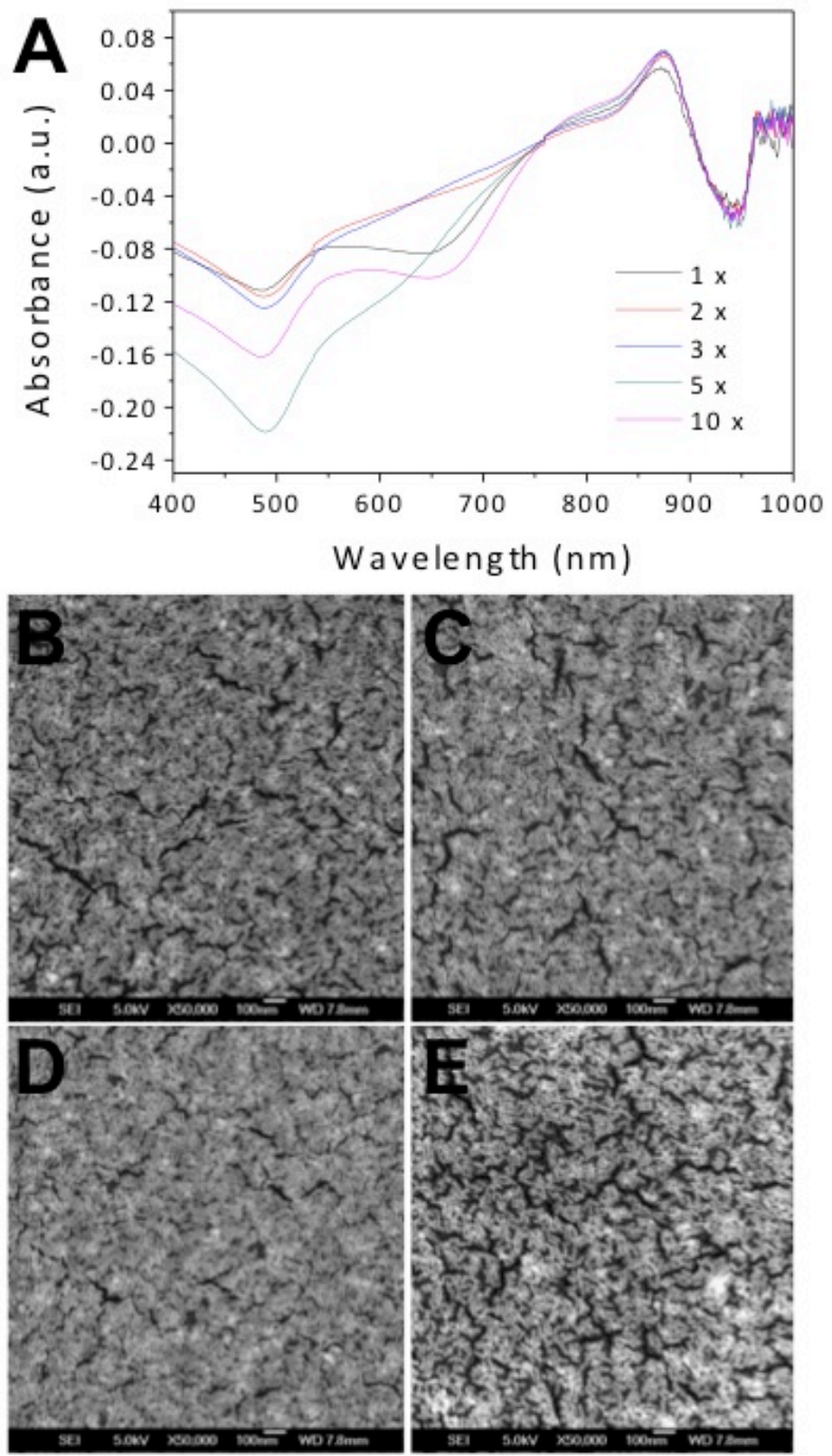

Figure 8-10. (A) UV-vis-NIR spectra obtained from POEGMA-grown glass coverslips after $24 \mathrm{~h}$ immersion in AuNR solution with increasing AuNR concentration. SEM images obtained from POEGMA-grown substrates after $24 \mathrm{~h}$ immersion in AuNR solution with (B) twice, (C) thrice, (D) 5 times and (E) 10 times higher concentration than initially prepared. 


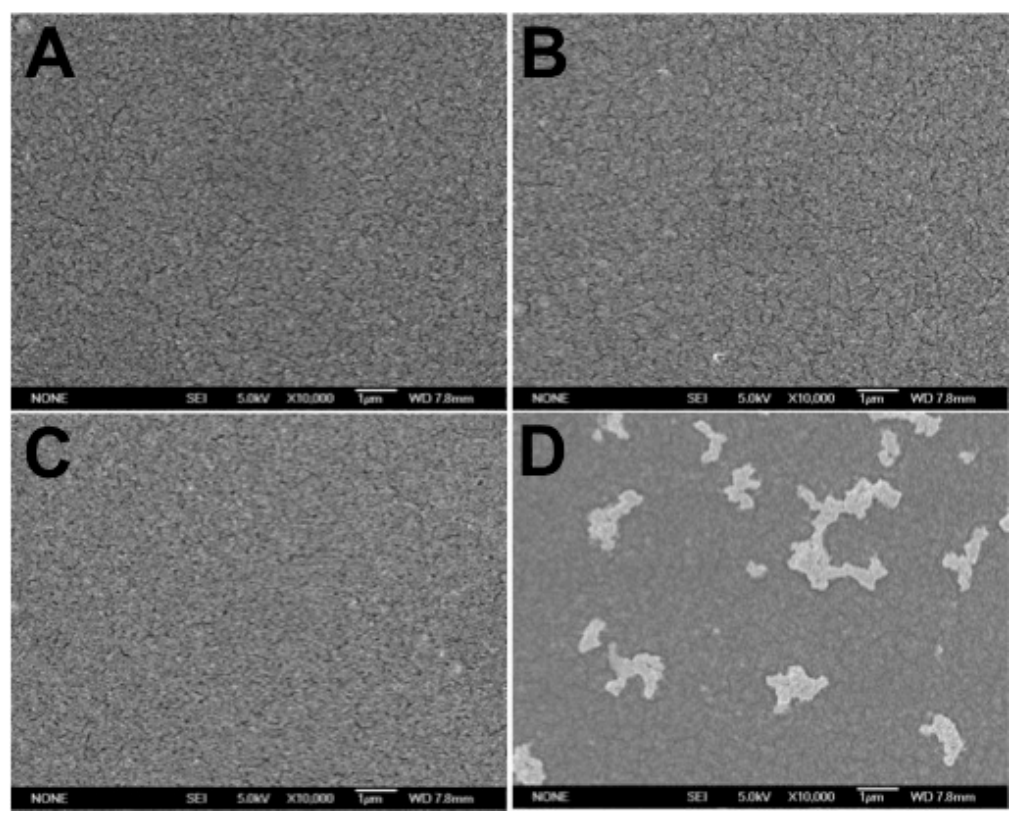

Figure 8-11. Lower magnification (10,000x) SEM images obtained from POEGMAgrown substrates after $24 \mathrm{~h}$ immersion in AuNR solution with (A) twice, (B) thrice, (C) 5 times and (D) 10 times higher concentration than initially prepared.

Therefore, we eventually used five-fold concentrated AuNR solution and found that a uniform AuNR super-aggregate with full coverage could be attained even with 6 $\mathrm{h}$ immersion time. To prove that the super-aggregate is three-dimensional and floating on the substrate, we compared the assembly with AuNRs deposited on polystyrenesulfonate (PSS)-treated substrates (AuNR-PSS) via overnight immersion. Top-down SEM images of AuNR-PSS show satisfactory uniformity without full coverage (Figures 8-12A \& B). Cross-sectional SEM images taken at $45^{\circ}$ tilt angle (Figures 8-12C \& D) along with a 3D AFM image (Figures 8-12E) clearly show the AuNRs deposited flat on the substrate resulting in a two-dimensional, monolayer type of assembly. On the other hand, top-down SEM images of AuNR super-aggregate show excellent uniformity with full coverage over the entire observed area (Figures 812F \& G). Cross-sectional SEM images show that the thickness of the superaggregate is homogeneous (Figures 8-12H) and the AuNRs are not deposited flat on 
the brush surface but are closely packed comprising of several tilted, slipped and overlapping AuNRs (Figure 8-12I). Such arrangement may prove to be highly beneficial in SERS since a recent simulation study had shown that the highest SERS enhancement factors could be obtained from slipped AuNR quartets ${ }^{362}$. Such variety of configurations is possible owing to the depth of the brush, which at the same time provides buoyancy, resulting in the entire ensemble being elevated from the substrate surface. Interestingly, when checked under atomic force microscopy (AFM) (Figure 8-12J), we found that the surface morphology of the assembly is smooth, unlike the case of AuNR-PSS where AuNRs appear protruded. This confirmed that the AuNRs are actually floating on the sub-surface of the POEGMA brush.
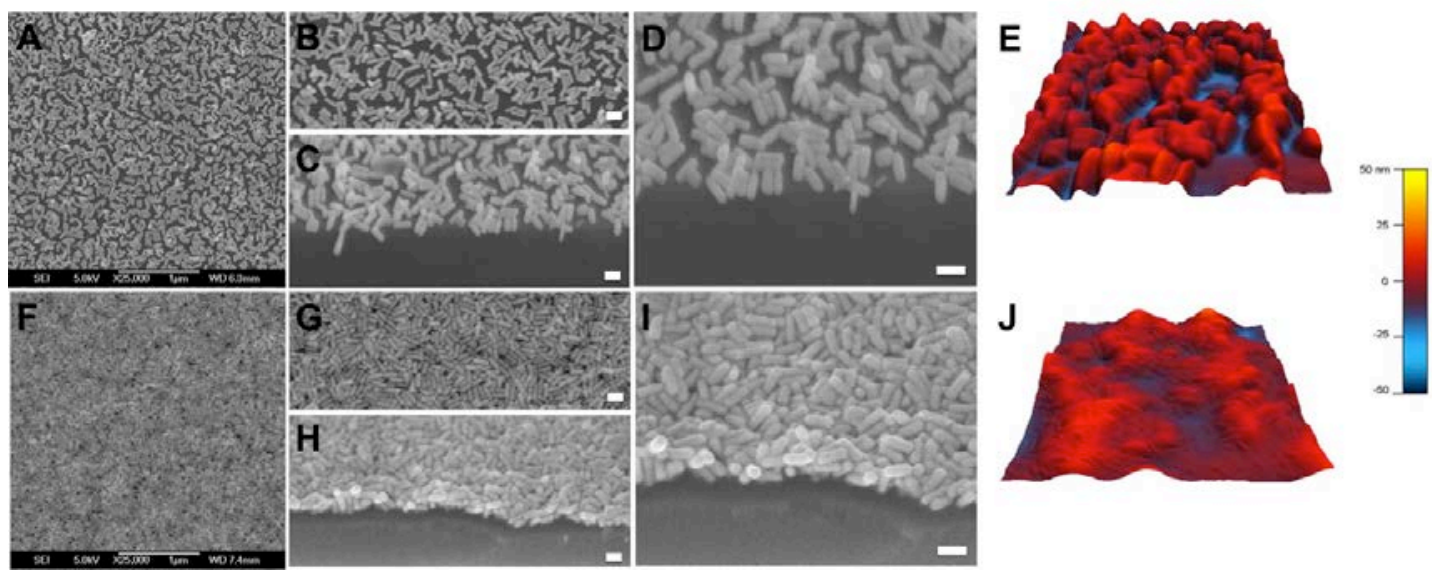

Figure 8-12. SEM images of PSS-treated silicon substrate after immersion in 5 times concentrated AuNR solution overnight viewed (A-B) top-down at increasing magnification, (C-D) at $45^{\circ}$-tilt angle at increasing magnification along with (E) 3D AFM image taken with a scan size of $1 \mu \mathrm{m}^{2}$. SEM images of POEGMA-grown silicon substrate after immersion in 5 times concentrated AuNR solution for $6 \mathrm{~h}$ viewed (F-G) top-down at increasing magnification, (H-I) at $45^{\circ}$-tilt angle at increasing magnification along with (J) 3D AFM image taken with a scan size of $1 \mu \mathrm{m}^{2}$. 


\subsubsection{SERS enhancement capability of gold nanorod-incorporated POEGMA}

We then investigated the SERS enhancement capability of the AuNR superaggregates using rhodamine $6 \mathrm{G}(\mathrm{R} 6 \mathrm{G})$ as the probe molecule and found that the SERS signal was highest using a $633 \mathrm{~nm}$ laser source (Figure 8-13). This may be somewhat surprising considering the fact that the LSPR peak from the UV-vis spectra lies at around $850 \mathrm{~nm}$. While there are several opinions suggesting that maximum SERS enhancement occurs when the excitation wavelength is close, or slightly blue-shifted, to the LSPR peak ${ }^{363-366}$, this usually holds true when the arrangement of nanostructures is non-complicated such as in well-defined arrays. For the case of nanoparticle aggregates however, several other works ${ }^{367-369}$ have shown that it is difficult to pinpoint any strong correlation between the excitation wavelength and the UV-vis extinction, in their contribution towards maximum SERS enhancement. On the other hand, it was found that the gap sizes as well as the number of nanoparticles that make up the aggregates represent a more dominating factor ${ }^{367}$. It was also shown that even for simple nanoparticle dimers and trimers, the LSPR peak does not represent a good indicator for the best excitation wavelength ${ }^{368}$. Furthermore in our case, we are essentially looking at a long-range, super-aggregate of nanorods, arranged in a variety of configurations. Unlike the UV-vis extinction spectra, which arise from two equally effective contributions namely 1) collective localized surface plasmon resonances from individual nanorods and 2) plasmon-coupled resonances from two or more neighbouring nanorods, SERS spectra arise predominantly from electromagnetic field enhancements at the junctions between nanorods. In other words, the contribution for enhanced SERS intensities mainly orginate from probe molecules localized between these junctions, even though there are other probe molecules encapsulating the 
nanorods. This introduces another degree of complexity since, particularly in our case, the junctions exist in a huge variety of configuration ranging from AuNRs arranged end-to-end, end-to-side to side-to-side, as well as those meeting at other intermediary angles. And given the fact that gap size plays an important role SERS enhancement, the gap sizes in our work are also wide-ranging, with most nanorods almost touching each other. Taken together, these complicating factors make it less likely that the LSPR peak from the UV-vis spectra would make a good predictor of the excitation wavelength for highest SERS enhancement; consistent with previous works in literature $367,369,370$.

Compared to two-dimensional AuNR assembly on PSS, the signal intensity from $1 \mu \mathrm{M}$ R6G was 15 times higher (Figure 8-14A). We then obtained SERS spectra from decreasing R6G concentrations (Figure 8-14B) and found that the peaks could still be resolved down to a concentration of $10^{-16} \mathrm{M}$ (Figure 8-14C), suggesting the high SERS sensitivity of the AuNR super-aggregates. This detection limit is among the lowest compared to other platforms developed recently ${ }^{371-374}$. Further reduction to $10^{-}$ ${ }^{17}$ and $10^{-18} \mathrm{M}$ R6G did not result in any resolvable signal. As SERS signal intensities have the tendency to fluctuate when acquired from different points even with the slightest change in position, the uniformity of the AuNR-POEGMA over the entire silicon substrate was briefly inspected using the optical microscope attached to the Raman spectrometer before 15 random points was chosen for the acquisition of Raman signals. The Raman signals from these points are shown in Figure 8-15. The relative standard deviation (RSD), which is indicative of the degree of signal intensity variations, was calculated from the most intense peak (i.e. at $\sim 1514 \mathrm{~cm}^{-1}$ ) of each signal. An RSD value of $6.72 \%$ was obtained. 
Finally, we also checked the effect of long-term storage and found that exposing the platform in air for more than a month would degrade the SERS signal by $43.77 \%$, almost half the signal obtained from freshly prepared samples (Figure 8-16). As AuNRs would have remained intact over this period, we believe the signal degradation is due to the tendency of POEGMA to be oxidized in air, resulting in the reduced swelling capability of the brush. As a consequence, probe molecules have reduced access to the AuNR surface since the AuNRs cannot space out when immersed in solution. This demonstrates the important role of POEGMA, especially in providing nanomobility to the super-aggregates, and eventually determining the sensing performance of the platform.

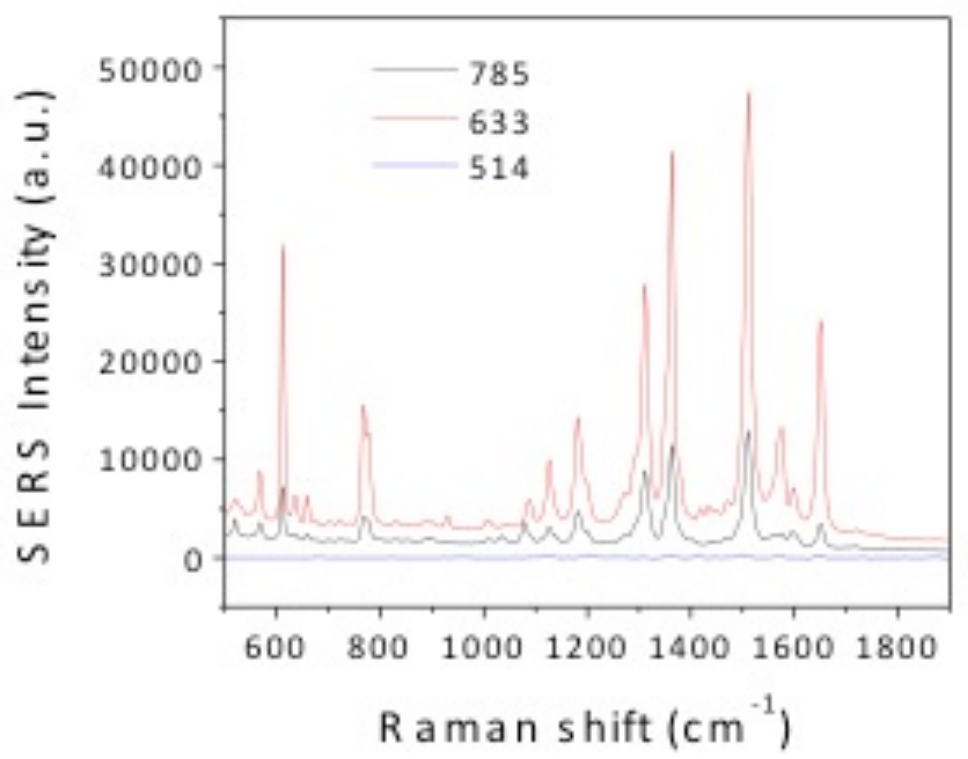

Figure 8-13. A comparison of SERS signal intensities from AuNR-POEGMA obtained with $1 \mu \mathrm{M}$ of R6G using laser sources of different wavelengths; 514, 633 and $785 \mathrm{~nm}$. 

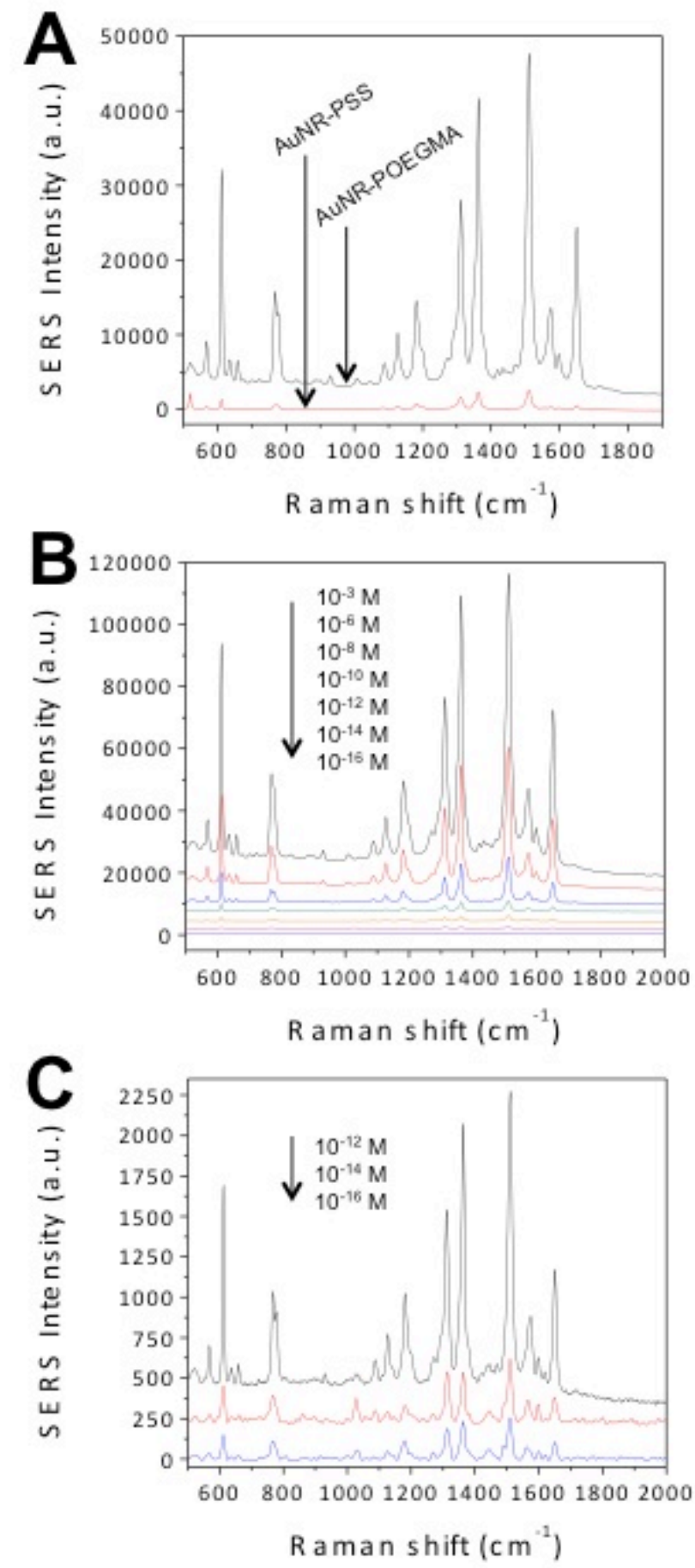

Figure 8-14. (A) A comparison of SERS signal intensities obtained from AuNR-PSS and AuNR-POEGMA silicon substrates with $1 \mu \mathrm{M}$ of R6G. (B) SERS spectra obtained from AuNR-POEGMA silicon substrates with decreasing concentrations of R6G from $10^{-3} \mathrm{M}$ to $10^{-16} \mathrm{M}$; (C) enlarged spectra from $10^{-12} \mathrm{M}$ to $10^{-16} \mathrm{M}$ R6G. 


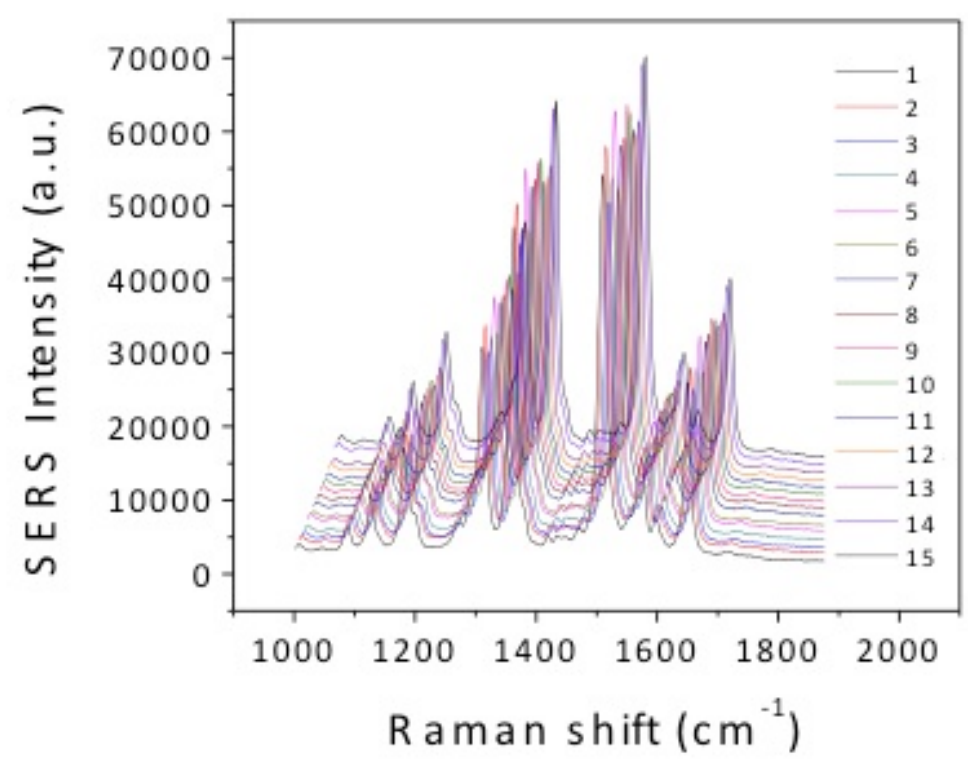

Figure 8-15. SERS spectra obtained from 15 random points of AuNR-POEGMA silicon substrates with $1 \mu \mathrm{M}$ R6G.

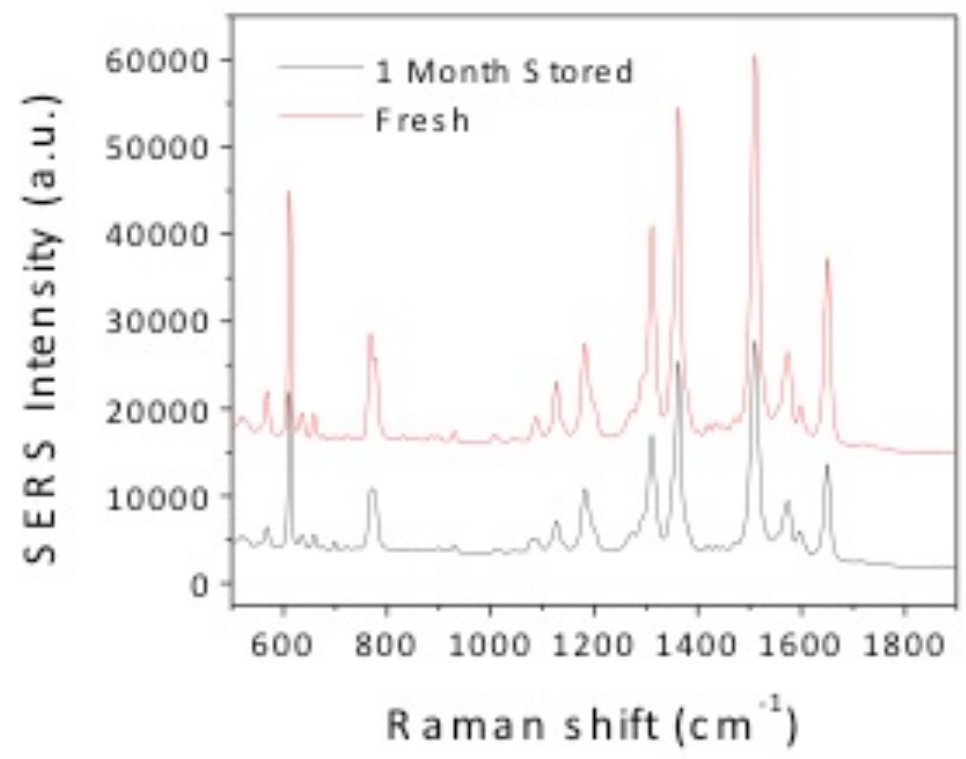

Figure 8-16. SERS spectra obtained from fresh and stored AuNR-POEGMA silicon substrates with $1 \mu \mathrm{M}$ R6G. 


\subsection{Conclusion}

We established a new type of high-density, closely packed two- to threedimensional AuNR assemblies resembling floating super-aggregates on a substrate. The assemblies were obtained in a single step by fully immersing POEGMA-grown glass and silicon substrates in as-prepared AuNR solution. Good lateral distribution and homogeneous thickness was observed over macroscopic distances resulting in extensive long-range coupling. As a consequence, a distinct sharp LSPR absorbance peak formed at around $875 \mathrm{~nm}$, in addition to the conventional transverse and longitudinal peaks. The assemblies were dynamic due to the swellability of POEGMA, allowing the AuNRs to space out when in solution and collapse when dry, resulting in better access of SERS probe molecules to the AuNR surface. Consequently, this increased the amount of probe molecules within a nanorod-nanorod junction upon drying. Compared to a rigid substrate, the improvement in terms of SERS signal intensity is 15 -fold. We believe that such dynamic super-aggregates could soon be found in devices suitable for consumer use since the fabrication method is extremely simple and can be transferred into a large-scale process without the need for technologically advanced equipments. In addition, our floating AuNR superaggregates could also be integrated into microscale three-dimensional scaffolds recently published such as the SiNW arrays simply by growing POEGMA on the SiNW surface, or any other microstructure for that matter. 


\section{Chapter 9}

\section{Conclusion and Future Direction}

\subsection{Conclusion}

We have shown that the POEGMA brush possesses non-fouling properties and is capable of immobilizing antibodies through physical immobilization. Depending on the polymer brush thickness, we found that antibodies can be either be embedded or partially infiltrated at the sub-surface of the brush. Apart from proteins and antibodies, metal nanoparticles can also be adsorbed, embedded and infiltrated within the brush.

We have shown that the combination of polymer brush and gold nanoparticles can result in new types of chemical and biosensing platforms. The gold nanoparticles can easily be incorporated into the brush without any form of chemical modification allowing the retention of the non-fouling property. With respect to gold nanospheres, there are a few ways how they can be assembled on the brush depending on their size. Large size gold nanoparticles typically adsorb on the surface of the brush while small size gold nanoparticles can easily infiltrate into the depth of the brush. Intermediate size gold nanoparticles can be arranged in a three-dimensional fashion by means of a novel method referred to as 'in-stacking'. This method exploits the swellability of the brush; based on repeated drying and immersion in gold nanoparticle solution, the nanoparticles are forced to squeeze into the depth of the brush.

Since the gold nanoparticles are attached to the brush without any strong covalent attachment schemes, the nanoparticles can be modified downstream for sensing purposes. The absence of strong covalent attachment also means that the gold nanoparticles can be mobilized within the brush. This is exploited for lead ion and 
thrombin sensing by means of modification of the gold nanoparticles with thiosulfate and thiolated aptamer respectively. In the case of lead ion detection, small gold nanoparticles infiltrated into the brush were released in the presence of lead ion. In the case of thrombin detection, the presence of thrombin triggers the rearrangement of gold nanoparticles. Both of these changes could be detected using UV-vis spectrophotometer and even via naked eye.

We found that while gold nanospheres, prepared by citrate reduction method can be easily adsorbed on the brush, gold nanorods which involved surfactants cannot be readily adsorbed on the brush. By performing a dedicated study to investigate how gold nanorods can be immobilized on a surface, we found that the assembly density of the gold nanorods on a surface treated with APTES and PSS can be tuned simply by adjusting the ionic strength of the gold nanorod solution. We arrive at the conclusion that there is a double shielding effect leading to the neutralization of charges around the gold nanorods. This same principle was used to immobilize gold nanorods on polymer brushes. However, for the case of polymer brushes, since there is vertical depth involved, the arrangement of gold nanorods is more well packed and resulted in a three-dimensional assembly. Gold nanorods on polymer brushes were eventually used for SERS application and found that Rhodamine $6 \mathrm{G}$ could be detected down to sub-femtomolar levels. More importantly, we demonstrated that the assembly is uniformed in the large scale and that we could easily transfer the platform to a glass slide.

Most importantly, the contribution in this thesis demonstrates that the formation of sensitive biosensing platforms could be achieved via a simple route; by immersion of POEGMA-treated substrates in as-prepared gold nanoparticle solution. Such combination has resulted in a new class of non-rigid nanocomposite material that 
allows one element (i.e. gold nanoparticle) to be controllably dissociated from the host material (i.e. polymer brush). Such simple approach coupled with the freedom and flexibility to utilize in various plasmonic based sensing platform (e.g. UV-vis and SERS), be it in a small-sized substrate or large-sized substrate, would accelerate the development of low-cost biosensors.

\subsection{Future works}

With regard to the application of gold nanoparticle polymer brush composites for the purpose of chemical and biomolecular sensing, a foreseeable limitation may arise from the degradation of polymer brush when it is exposed to air, which in turn is due to POEGMA being prone to oxidation. This may lead to the reduction in non-fouling behaviour as well as the reduction in swelling capability, as evident in Chapter 7. Consequently, this may adversely affect the sensitivity of the sensing platform, since the mobility of gold nanoparticles is reduced. For the purpose of practical application and device fabrication, it is therefore of immediate concern to determine the precautions that need to be taken with regard to long-term storage to ensure that the performance does not vary from freshly-prepared. This may include passivating the asprepared composites by means of introducing other functional groups into the system or protecting the as-prepared composites by means of storing it in inert solutions prior to sensing. Such detailed works need to be performed meticulously not only to establish all possible factors that could contribute to degradation but also to quantify the rate of degradation as well as to ensure the effectiveness of any introduced precautionary measures.

In terms of sensor design, the future direction of polymer brush nanoparticle composite system that could culminate from this work includes the development of point-of-care diagnostic systems based on reversible gold nanoparticle mobility on the 
brush. So far, we have established that gold nanoparticles within polymer brush can be easily post-functionalized and demonstrated the feasibility of triggered, controlled release of gold nanoparticle from the brush. As we show that both complete and partial release are possible, more work can be done to fine-tune the interactions between the nanoparticle and the brush to ensure that the nanoparticles not only have the freedom to escape or move around on the brush within a fixed region, but also have the ability to move, reversibly, in a concerted manner. This would eventually allow switchable and controlled aggregation in the form of dimer, trimer and multimer arrays. Once methodologies for controlled placement and reversible movement of nanoparticles within the polymer brush has been established, the combination of several nanoparticles of different morphologies to form unique structures such as nanodumbbells can be explored to improve the sensing performance. As discussed earlier in Chapter 2, the feasibility of shape-shifting nanoparticles, within the polymer brush can also be investigated.

Other directions that could be undertaken include the combination of our approaches with micro-patterned surfaces. So far, we have demonstrated our system on bulk planar surfaces. By extending our techniques on substrates with patterned surfaces, the surface area could be increased and sensitivity may be improved. This is particularly relevant for the case of detection based on SERS, where an increased surface area and an abundance of nanostructure junctions are particularly beneficial in increasing the exposed area of laser illumination and increasing the degree of SERS enhancement, respectively.

Due to the simplicity of the approach and commonality of the underlying substrate, which is mostly glass, all these sensing platforms could be integrated within a compact microfluidic setup for low-cost sensing device fabrication. Such integration 
would eventually allow direct chemical and biomolecular detection from complex solutions, without the need for pre-treatments, for various environmental and healthcare applications, ranging from water quality checks to early detection of diseases. 


\section{BIBLIOGRAPHY}

1. Chen, T., Amin, I. \& Jordan, R. Patterned polymer brushes. Chemical Society Reviews 41, 3280-3296 (2012).

2. Zhou, X., Liu, X., Xie, Z. \& Zheng, Z. 3D-patterned polymer brush surfaces. Nanoscale 3, 4929-4939 (2011).

3. Azzaroni, O. Polymer brushes here, there, and everywhere: Recent advances in their practical applications and emerging opportunities in multiple research fields. Journal of Polymer Science Part A: Polymer Chemistry 50, 3225-3258 (2012).

4. Yan, H. et al. A high-mobility electron-transporting polymer for printed transistors. Nature 457, 679-686 (2009).

5. Decher, G. Fuzzy Nanoassemblies: Toward Layered Polymeric Multicomposites. Science 277, 1232-1237 (1997).

6. Milner, S.T. Polymer Brushes. Science 251, 905-914 (1991).

7. Edmondson, S., Osborne, V.L. \& Huck, W.T.S. Polymer brushes via surfaceinitiated polymerizations. Chemical Society Reviews 33, 14-22 (2004).

8. Wang, J.-S. \& Matyjaszewski, K. Controlled/"living" radical polymerization. atom transfer radical polymerization in the presence of transition-metal complexes. Journal of the American Chemical Society 117, 5614-5615 (1995).

9. Huang, X. \& Wirth, M.J. Surface-Initiated Radical Polymerization on Porous Silica. Analytical Chemistry 69, 4577-4580 (1997).

10. Lai, P.Y. \& Halperin, A. Polymer brush at high coverage. Macromolecules 24, 4981-4982 (1991). 
11. Zhulina, E.B., Borisov, O.V., Pryamitsyn, V.A. \& Birshtein, T.M. Coil-globule type transitions in polymers. 1. Collapse of layers of grafted polymer chains. Macromolecules 24, 140-149 (1991).

12. Krishnamoorthy, M., Hakobyan, S., Ramstedt, M. \& Gautrot, J.E. SurfaceInitiated Polymer Brushes in the Biomedical Field: Applications in Membrane Science, Biosensing, Cell Culture, Regenerative Medicine and Antibacterial Coatings. Chemical Reviews 114, 10976-11026 (2014).

13. Hadjesfandiari, N., Yu, K., Mei, Y. \& Kizhakkedathu, J.N. Polymer brushbased approaches for the development of infection-resistant surfaces. Journal of Materials Chemistry B 2, 4968-4978 (2014).

14. Van Kampen, C.L., Gibbons, D.F. \& Jones, R.D. Effect of implant surface chemistry upon arterial thrombosis. Journal of Biomedical Materials Research 13, 517-541 (1979).

15. Hayashi, K., Fukumura, H. \& Yamamoto, N. In vivo thrombus formation induced by complement activation on polymer surfaces. Journal of Biomedical Materials Research 24, 1385-1395 (1990).

16. Helmus, M.N., Gibbons, D.F. \& Jones, R.D. The effect of surface charge on arterial thrombosis. Journal of Biomedical Materials Research 18, 165-183 (1984).

17. An, Y.H. \& Friedman, R.J. Concise review of mechanisms of bacterial adhesion to biomaterial surfaces. Journal of Biomedical Materials Research 43, 338-348 (1998).

18. Hetrick, E.M. \& Schoenfisch, M.H. Reducing implant-related infections: active release strategies. Chemical Society Reviews 35, 780-789 (2006). 
19. Gottenbos, B., van der Mei, H.C. \& Busscher, H.J. Initial adhesion and surface growth of Staphylococcus epidermidis and Pseudomonas aeruginosa on biomedical polymers. Journal of Biomedical Materials Research 50, 208-214 (2000).

20. Kambic, H.E., Murabayashi, S. \& Nose, Y. Biomaterials in Artificial Organs. Chemical \& Engineering News Archive 64, 30-48 (1986).

21. Jensen, L.O. et al. Stent Thrombosis, Myocardial Infarction, and Death After Drug-Eluting and Bare-Metal Stent Coronary Interventions. Journal of the American College of Cardiology 50, 463-470 (2007).

22. Tengvall, P., Lundstrom, I., Sjoqvist, L., Elwing, H. \& Bjursten, L.M. Titanium-hydrogen peroxide interaction: model studies of the influence of the inflammatory response on titanium implants. Biomaterials 10, 166-175 (1989).

23. Norde, W. Adsorption of proteins from solution at the solid-liquid interface. Advances in Colloid and Interface Science 25, 267-340 (1986).

24. Giacomelli, C.E., Bremer, M.G.E.G. \& Norde, W. ATR-FTIR Study of IgG Adsorbed on Different Silica Surfaces. Journal of Colloid and Interface Science 220, 13-23 (1999).

25. Norde, W. \& Giacomelli, C.E. BSA structural changes during homomolecular exchange between the adsorbed and the dissolved states. Journal of Biotechnology 79, 259-268 (2000).

26. Haynes, C.A. \& Norde, W. Globular proteins at solid/liquid interfaces. Colloids and Surfaces B: Biointerfaces 2, 517-566 (1994).

27. Giacomelli, C.E. \& Norde, W. The Adsorption-Desorption Cycle. Reversibility of the BSA-Silica System. Journal of Colloid and Interface Science 233, 234$240(2001)$. 
28. Wertz, C.F. \& Santore, M.M. Adsorption and Relaxation Kinetics of Albumin and Fibrinogen on Hydrophobic Surfaces: Single-Species and Competitive Behavior. Langmuir 15, 8884-8894 (1999).

29. Wertz, C.F. \& Santore, M.M. Adsorption and Reorientation Kinetics of Lysozyme on Hydrophobic Surfaces. Langmuir 18, 1190-1199 (2002).

30. Wertz, C.F. \& Santore, M.M. Effect of Surface Hydrophobicity on Adsorption and Relaxation Kinetics of Albumin and Fibrinogen: Single-Species and Competitive Behavior. Langmuir 17, 3006-3016 (2001).

31. Wertz, C.F. \& Santore, M.M. Fibrinogen Adsorption on Hydrophilic and Hydrophobic Surfaces - Geometrical and Energetic Aspects of Interfacial Relaxations. Langmuir 18, 706-715 (2002).

32. Santore, M.M. \& Wertz, C.F. Protein Spreading Kinetics at Liquid-Solid Interfaces via an Adsorption Probe Method. Langmuir 21, 10172-10178 (2005).

33. Buijs, J., van den Berg, P.A.W., Lichtenbelt, J.W.T., Norde, W. \& Lyklema, J. Adsorption Dynamics of IgG and Its F(ab')2 and Fc Fragments Studied by Reflectometry. Journal of Colloid and Interface Science 178, 594-605 (1996).

34. Buijs, J., Lichtenbelt, J.W.T., Norde, W. \& Lyklema, J. Adsorption of monoclonal IgGs and their F(ab')2 fragments onto polymeric surfaces. Colloids and Surfaces B: Biointerfaces 5, 11-23 (1995).

35. Norde, W. \& Lyklema, J. Interfacial behaviour of proteins, with special reference to immunoglobulins. A physicochemical study. Advances in Colloid and Interface Science 179-182, 5-13 (2012). 
36. Buijs, J., White, D.D. \& Norde, W. The effect of adsorption on the antigen binding by $\operatorname{IgG}$ and its $\mathrm{F}\left(\mathrm{ab}^{\prime}\right) 2$ fragments. Colloids and Surfaces $B$ : Biointerfaces 8, 239-249 (1997).

37. Norde, W. \& Lyklema, J. Protein adsorption and bacterial adhesion to solid surfaces: A colloid-chemical approach. Colloids and Surfaces 38, 1-13 (1989).

38. Rijnaarts, H.H.M., Norde, W., Lyklema, J. \& Zehnder, A.J.B. DLVO and steric contributions to bacterial deposition in media of different ionic strengths. Colloids and Surfaces B: Biointerfaces 14, 179-195 (1999).

39. Baszkin, A. \& Lyman, D.J. The interaction of plasma proteins with polymers. I. Relationship between polymer surface energy and protein adsorption/desorption. Journal of Biomedical Materials Research 14, 393-403 (1980).

40. Brash, J.L. \& Lyman, D.J. Adsorption of plasma proteins in solution to uncharged, hydrophobic polymer surfaces. Journal of Biomedical Materials Research 3, 175-189 (1969).

41. Chiu, T.C., Craig Metcalf, L. \& Lyman, D.J. Electrophoretic analysis of protein adsorbed on polymer surfaces. Journal of Biomedical Materials Research 15, 781-784 (1981).

42. van Wachem, P.B. et al. Adhesion of cultured human endothelial cells onto methacrylate polymers with varying surface wettability and charge. Biomaterials 8, 323-328 (1987).

43. van Wachem, P.B. et al. Interaction of cultured human endothelial cells with polymeric surfaces of different wettabilities. Biomaterials 6, 403-408 (1985). 
44. van Wachem, P.B. et al. The influence of protein adsorption on interactions of cultured human endothelial cells with polymers. Journal of Biomedical Materials Research 21, 701-718 (1987).

45. Ratner, B.D., Horbett, T., Hoffman, A.S. \& Hauschka, S.D. Cell adhesion to polymeric materials: Implications with respect to biocompatibility. Journal of Biomedical Materials Research 9, 407-422 (1975).

46. Vroman, L., Adams, A.L., Fischer, G.C. \& Munoz, P.C. Interaction of high molecular weight kininogen, factor XII, and fibrinogen in plasma at interfaces. Blood 55, 156-159 (1980).

47. Elwing, H. Protein absorption and ellipsometry in biomaterial research. Biomaterials 19, 397-406 (1998).

48. Tengvall, P., Lundstrom, I. \& Liedberg, B. Protein adsorption studies on model organic surfaces: an ellipsometric and infrared spectroscopic approach. Biomaterials 19, 407-422 (1998).

49. Vroman, L. \& Adams, A.L. Findings with the recording ellipsometer suggesting rapid exchange of specific plasma proteins at liquid/solid interfaces. Surface Science 16, 438-446 (1969).

50. Stanford, M.F., Munoz, P.C. \& Vroman, L. Platelets adhere where flow has left fibrinogen on glass. Annals of the New York Academy of Sciences 416, 504-512 (1983).

51. Vroman, L. \& Adams, A.L. Adsorption of proteins out of plasma and solutions in narrow spaces. Journal of Colloid and Interface Science 111, 391-402 (1986).

52. Vroman, L. Effect of Adsorbed Proteins on the Wettability of Hydrophilic and Hydrophobic Solids. Nature 196, 476-477 (1962). 
53. Barnthip, N., Parhi, P., Golas, A. \& Vogler, E.A. Volumetric interpretation of protein adsorption: Kinetics of protein-adsorption competition from binary solution. Biomaterials 30, 6495-6513 (2009).

54. Noh, H. \& Vogler, E.A. Volumetric interpretation of protein adsorption: Partition coefficients, interphase volumes, and free energies of adsorption to hydrophobic surfaces. Biomaterials 27, 5780-5793 (2006).

55. Noh, H. \& Vogler, E.A. Volumetric interpretation of protein adsorption: Mass and energy balance for albumin adsorption to particulate adsorbents with incrementally increasing hydrophilicity. Biomaterials 27, 5801-5812 (2006).

56. Noh, H. \& Vogler, E.A. Volumetric interpretation of protein adsorption: Competition from mixtures and the Vroman effect. Biomaterials 28, 405-422 (2007).

57. Noh, H., Yohe, S.T. \& Vogler, E.A. Volumetric interpretation of protein adsorption: Ion-exchange adsorbent capacity, protein $\mathrm{pI}$, and interaction energetics. Biomaterials 29, 2033-2048 (2008).

58. Parhi, P., Golas, A., Barnthip, N., Noh, H. \& Vogler, E.A. Volumetric interpretation of protein adsorption: Capacity scaling with adsorbate molecular weight and adsorbent surface energy. Biomaterials 30, 6814-6824 (2009).

59. Baier, R.E., Shafrin, E.G. \& Zisman, W.A. Adhesion: Mechanisms That Assist or Impede It. Science 162, 1360-1368 (1968).

60. Baier, R.E., Meyer, A.E., Natiella, J.R., Natiella, R.R. \& Carter, J.M. Surface properties determine bioadhesive outcomes: Methods and results. Journal of Biomedical Materials Research 18, 337-355 (1984).

61. Baier, R.E. Conditioning Surfaces to Suit the Biomedical Environment: Recent Progress. Journal of Biomechanical Engineering 104, 257-271 (1982). 
62. Ruckenstein, E., Gourisankar, S. \& Baier, R.E. A nondestructive approach to characterize deposits on various surfaces. Journal of Colloid and Interface Science 96, 245-250 (1983).

63. Baier, R.E. The organization of blood components near interfaces. Annals of the New York Academy of Sciences 283, 17-36 (1977).

64. Liedberg, B., Nylander, C. \& Lunstrom, I. Surface plasmon resonance for gas detection and biosensing. Sensors and Actuators 4, 299-304 (1983).

65. Jonsson, U. et al. Real-Time Biospecific Interaction Analysis Using SurfacePlasmon Resonance and A Sensor Chip Technology. Biotechniques 11, 620-\& (1991).

66. Stenberg, E., Persson, B., Roos, H. \& Urbaniczky, C. Quantitative determination of surface concentration of protein with surface plasmon resonance using radiolabeled proteins. Journal of Colloid and Interface Science 143, 513-526 (1991).

67. Lofas, S. \& Johnsson, B. A novel hydrogel matrix on gold surfaces in surface plasmon resonance sensors for fast and efficient covalent immobilization of ligands. Journal of the Chemical Society, Chemical Communications, 15261528 (1990).

68. Johnsson, B., Lofas, S. \& Lindquist, G. Immobilization of proteins to a carboxymethyldextran-modified gold surface for biospecific interaction analysis in surface plasmon resonance sensors. Analytical Biochemistry 198, 268-277 (1991).

69. Liedberg, B., Lundstrom, I. \& Stenberg, E. Principles of biosensing with an extended coupling matrix and surface plasmon resonance. Sensors and Actuators B: Chemical 11, 63-72 (1993). 
70. Park, K., Mao, F.W. \& Park, H. The minimum surface fibrinogen concentration necessary for platelet activation on dimethyldichlorosilanecoated glass. Journal of Biomedical Materials Research 25, 407-420 (1991).

71. Harder, P., Grunze, M., Dahint, R., Whitesides, G.M. \& Laibinis, P.E. Molecular Conformation in Oligo(ethylene glycol)-Terminated SelfAssembled Monolayers on Gold and Silver Surfaces Determines Their Ability To Resist Protein Adsorption. The Journal of Physical Chemistry B 102, 426436 (1998).

72. Lestelius, M., Liedberg, B. \& Tengvall, P. In Vitro Plasma Protein Adsorption on w-Functionalized Alkanethiolate Self-Assembled Monolayers. Langmuir 13, 5900-5908 (1997).

73. Tosatti, S. et al. Peptide functionalized poly(l-lysine)-g-poly(ethylene glycol) on titanium: resistance to protein adsorption in full heparinized human blood plasma. Biomaterials 24, 4949-4958 (2003).

74. Benesch, J. et al. Protein adsorption to oligo(ethylene glycol) self-assembled monolayers: Experiments with fibrinogen, heparinized plasma, and serum. Journal of Biomaterials Science, Polymer Edition 12, 581-597 (2001).

75. Gombotz, W.R., Guanghui, W., Horbett, T.A. \& Hoffman, A.S. Protein adsorption to poly(ethylene oxide) surfaces. Journal of Biomedical Materials Research 25, 1547-1562 (1991).

76. Prime, K.L. \& Whitesides, G.M. Adsorption of proteins onto surfaces containing end-attached oligo(ethylene oxide): a model system using selfassembled monolayers. Journal of the American Chemical Society 115, 1071410721 (1993). 
77. Ostuni, E., Chapman, R.G., Holmlin, R.E., Takayama, S. \& Whitesides, G.M. A Survey of Structure-Property Relationships of Surfaces that Resist the Adsorption of Protein. Langmuir 17, 5605-5620 (2001).

78. Chapman, R.G. et al. Polymeric Thin Films That Resist the Adsorption of Proteins and the Adhesion of Bacteria. Langmuir 17, 1225-1233 (2001).

79. Jeon, S.I., Lee, J.H., Andrade, J.D. \& De Gennes, P.G. Protein-surface interactions in the presence of polyethylene oxide: I. Simplified theory. Journal of Colloid and Interface Science 142, 149-158 (1991).

80. De Gennes, P.G. Polymer solutions near an interface. Adsorption and depletion layers. Macromolecules 14, 1637-1644 (1981).

81. De Gennes, P.G. Polymers at an interface. 2. Interaction between two plates carrying adsorbed polymer layers. Macromolecules 15, $492-500$ (1982).

82. Timothy, B.M., Samuel, J.L. \& Kinam, P. in Proteins at Interfaces II, Vol. 602 395-404 (American Chemical Society, 1995).

83. Malmsten, M., Emoto, K. \& Van Alstine, J.M. Effect of Chain Density on Inhibition of Protein Adsorption by Poly(ethylene glycol) Based Coatings. Journal of Colloid and Interface Science 202, 507-517 (1998).

84. Halperin, A. Polymer Brushes that Resist Adsorption of Model Proteins: Design Parameters. Langmuir 15, 2525-2533 (1999).

85. Unsworth, L.D., Sheardown, H. \& Brash, J.L. Protein Resistance of Surfaces Prepared by Sorption of End-Thiolated Poly(ethylene glycol) to Gold: Effect of Surface Chain Density. Langmuir 21, 1036-1041 (2005).

86. Feng, W., Zhu, S., Ishihara, K. \& Brash, J.L. Adsorption of Fibrinogen and Lysozyme on Silicon Grafted with Poly(2-methacryloyloxyethyl 
Phosphorylcholine) via Surface-Initiated Atom Transfer Radical Polymerization. Langmuir 21, 5980-5987 (2005).

87. Feng, W., Brash, J.L. \& Zhu, S. Non-biofouling materials prepared by atom transfer radical polymerization grafting of 2-methacryloloxyethyl phosphorylcholine: Separate effects of graft density and chain length on protein repulsion. Biomaterials 27, 847-855 (2006).

88. Herrwerth, S., Eck, W., Reinhardt, S. \& Grunze, M. Factors that Determine the Protein Resistance of Oligoether Self-Assembled Monolayers ,àí Internal Hydrophilicity, Terminal Hydrophilicity, and Lateral Packing Density. Journal of the American Chemical Society 125, 9359-9366 (2003).

89. Kingshott, P., Thissen, H. \& Griesser, H.J. Effects of cloud-point grafting, chain length, and density of PEG layers on competitive adsorption of ocular proteins. Biomaterials 23, 2043-2056 (2002).

90. Morra, M. On the molecular basis of fouling resistance. Journal of Biomaterials Science, Polymer Edition 11, 547-569 (2000).

91. Feldman, K., Hahner, G., Spencer, N.D., Harder, P. \& Grunze, M. Probing Resistance to Protein Adsorption of Oligo(ethylene glycol)-Terminated SelfAssembled Monolayers by Scanning Force Microscopy. Journal of the American Chemical Society 121, 10134-10141 (1999).

92. Pertsin, A.J. \& Grunze, M. Computer Simulation of Water near the Surface of Oligo(ethylene glycol)-Terminated Alkanethiol Self-Assembled Monolayers. Langmuir 16, 8829-8841 (2000).

93. Wang, R.L.C., Kreuzer, H.J. \& Grunze, M. Molecular Conformation and Solvation of Oligo(ethylene glycol)-Terminated Self-Assembled Monolayers 
and Their Resistance to Protein Adsorption. The Journal of Physical Chemistry B 101, 9767-9773 (1997).

94. Zhao, C., Li, L., Wang, Q., Yu, Q. \& Zheng, J. Effect of Film Thickness on the Antifouling Performance of Poly(hydroxy-functional methacrylates) Grafted Surfaces. Langmuir 27, 4906-4913 (2011).

95. Raynor, J.E., Petrie, T.A., García, A.J. \& Collard, D.M. Controlling Cell Adhesion to Titanium: Functionalization of Poly[oligo(ethylene glycol)methacrylate] Brushes with Cell-Adhesive Peptides. Advanced Materials 19, 1724-1728 (2007).

96. Wattendorf, U. \& Merkle, H.P. PEGylation as a tool for the biomedical engineering of surface modified microparticles. Journal of Pharmaceutical Sciences 97, 4655-4669 (2008).

97. Guo, W. et al. Switchable Bifunctional Stimuli-Triggered Poly-NIsopropylacrylamide/DNA Hydrogels. Angewandte Chemie International Edition 53, 10134-10138 (2014).

98. Okano, T., Yamada, N., Okuhara, M., Sakai, H. \& Sakurai, Y. Mechanism of cell detachment from temperature-modulated, hydrophilic-hydrophobic polymer surfaces. Biomaterials 16, 297-303 (1995).

99. Tang, H., Zhang, B. \& Wu, P. On the two-step phase transition behavior of the Poly(N-isopropylacrylamide) (PNIPAM) brush: different zones with different orders. Soft Matter 10, 7278-7284 (2014).

100. Kuang, J. \& Messersmith, P.B. Universal Surface-Initiated Polymerization of Antifouling Zwitterionic Brushes Using a Mussel-Mimetic Peptide Initiator. Langmuir 28, 7258-7266 (2012). 
101. Sin, M.-C., Chen, S.-H. \& Chang, Y. Hemocompatibility of zwitterionic interfaces and membranes. Polym J 46, 436-443 (2014).

102. Yoshimoto, K., Hirase, T., Madsen, J., Armes, S.P. \& Nagasaki, Y. NonFouling Character of Poly[2-(methacryloyloxy)ethyl Phosphorylcholine]Modified Gold Surfaces Fabricated by the 'Grafting to’ Method: Comparison of its Protein Resistance with Poly(ethylene glycol)-Modified Gold Surfaces. Macromolecular Rapid Communications 30, 2136-2140 (2009).

103. Zhang, Z. et al. Blood compatibility of surfaces with superlow protein adsorption. Biomaterials 29, 4285-4291 (2008).

104. Cheng, G. et al. Zwitterionic carboxybetaine polymer surfaces and their resistance to long-term biofilm formation. Biomaterials 30, 5234-5240 (2009).

105. Balazs, A.C., Emrick, T. \& Russell, T.P. Nanoparticle Polymer Composites: Where Two Small Worlds Meet. Science 314, 1107-1110 (2006).

106. Bockstaller, M.R., Mickiewicz, R.A. \& Thomas, E.L. Block Copolymer Nanocomposites: Perspectives for Tailored Functional Materials. Advanced Materials 17, 1331-1349 (2005).

107. Atwater, H.A. \& Polman, A. Plasmonics for improved photovoltaic devices. Nat Mater 9, 205-213 (2010).

108. Hu, M.-S. et al. Photosensitive gold-nanoparticle-embedded dielectric nanowires. Nat Mater 5, 102-106 (2006).

109. Wu, J.-L. et al. Surface Plasmonic Effects of Metallic Nanoparticles on the Performance of Polymer Bulk Heterojunction Solar Cells. ACS Nano 5, 959967 (2011).

110. Batson, P.E. Plasmonic Modes Revealed. Science 335, 47-48 (2012). 
111. Guo, L. \& Kim, D.-H. Reusable plasmonic aptasensors: using a single nanoparticle to establish a calibration curve and to detect analytes. Chemical Communications 47, 7125-7127 (2011).

112. Sapsford, K.E. et al. Functionalizing Nanoparticles with Biological Molecules: Developing Chemistries that Facilitate Nanotechnology. Chemical Reviews 113, 1904-2074 (2013).

113. Xia, F. et al. Colorimetric detection of DNA, small molecules, proteins, and ions using unmodified gold nanoparticles and conjugated polyelectrolytes. Proceedings of the National Academy of Sciences 107, 10837-10841 (2010).

114. Liu, J. \& Lu, Y. Accelerated Color Change of Gold Nanoparticles Assembled by DNAzymes for Simple and Fast Colorimetric Pb2+ Detection. Journal of the American Chemical Society 126, 12298-12305 (2004).

115. Jain, P.K., Eustis, S. \& El-Sayed, M.A. Plasmon Coupling in Nanorod Assemblies: Optical Absorption, Discrete Dipole Approximation Simulation, and Exciton-Coupling Model. The Journal of Physical Chemistry B 110, 18243-18253 (2006).

116. Chen, H., Shao, L., Li, Q. \& Wang, J. Gold nanorods and their plasmonic properties. Chemical Society Reviews 42, 2679-2724 (2013).

117. Ross, M.B., Blaber, M.G. \& Schatz, G.C. Using nanoscale and mesoscale anisotropy to engineer the optical response of three-dimensional plasmonic metamaterials. Nat Commun 5 (2014).

118. Huang, X., Neretina, S. \& El-Sayed, M.A. Gold Nanorods: From Synthesis and Properties to Biological and Biomedical Applications. Advanced Materials 21, 4880-4910 (2009). 
119. Chen, C.-D., Cheng, S.-F., Chau, L.-K. \& Wang, C.R.C. Sensing capability of the localized surface plasmon resonance of gold nanorods. Biosensors and Bioelectronics 22, 926-932 (2007).

120. Smith, A.M., Mancini, M.C. \& Nie, S. Bioimaging: Second window for in vivo imaging. Nat Nano 4, 710-711 (2009).

121. Tsai, M.-F. et al. Au Nanorod Design as Light-Absorber in the First and Second Biological Near-Infrared Windows for in Vivo Photothermal Therapy. ACS Nano 7, 5330-5342 (2013).

122. Gole, A. \& Murphy, C.J. Seed-Mediated Synthesis of Gold Nanorods: Role of the Size and Nature of the Seed. Chemistry of Materials 16, 3633-3640 (2004).

123. Grzelczak, M., Perez-Juste, J., Mulvaney, P. \& Liz-Marzan, L.M. Shape control in gold nanoparticle synthesis. Chemical Society Reviews 37, 17831791 (2008).

124. Xia, Y. et al. One-Dimensional Nanostructures: Synthesis, Characterization, and Applications. Advanced Materials 15, 353-389 (2003).

125. Horiuchi, S., Fujita, T., Hayakawa, T. \& Nakao, Y. Micropatterning of Metal Nanoparticles via UV Photolithography. Advanced Materials 15, 1449-1452 (2003).

126. Canelas, D.A., Herlihy, K.P. \& DeSimone, J.M. Top-down particle fabrication: control of size and shape for diagnostic imaging and drug delivery. Wiley Interdisciplinary Reviews: Nanomedicine and Nanobiotechnology 1, 391-404 (2009).

127. Yu, H.-D., Regulacio, M.D., Ye, E. \& Han, M.-Y. Chemical routes to topdown nanofabrication. Chemical Society Reviews 42, 6006-6018 (2013). 
128. Spatz, J.P. et al. A Combined Top-Down/Bottom-Up Approach to the Microscopic Localization of Metallic Nanodots. Advanced Materials 14, 18271832 (2002).

129. Diaz Fernandez, Y.A. et al. The conquest of middle-earth: combining topdown and bottom-up nanofabrication for constructing nanoparticle based devices. Nanoscale 6, 14605-14616 (2014).

130. Cui, Y. et al. Integration of Colloidal Nanocrystals into Lithographically Patterned Devices. Nano Letters 4, 1093-1098 (2004).

131. Cobley, C.M., Rycenga, M., Zhou, F., Li, Z.-Y. \& Xia, Y. Controlled Etching as a Route to High Quality Silver Nanospheres for Optical Studies. The Journal of Physical Chemistry C 113, 16975-16982 (2009).

132. Li, W. et al. Etching and Dimerization: A Simple and Versatile Route to Dimers of Silver Nanospheres with a Range of Sizes. Angewandte Chemie International Edition 49, 164-168 (2010).

133. Fan, N. et al. Selective Etching Induces Selective Growth and Controlled Formation of Various Platinum Nanostructures by Modifying Seed Surface Free Energy. ACS Nano 6, 4072-4082 (2012).

134. Xie, S. et al. Synthesis of Pd-Rh Core-Frame Concave Nanocubes and Their Conversion to Rh Cubic Nanoframes by Selective Etching of the Pd Cores. Angewandte Chemie International Edition 51, 10266-10270 (2012).

135. Lu, X. et al. Fabrication of Cubic Nanocages and Nanoframes by Dealloying $\mathrm{Au} / \mathrm{Ag}$ Alloy Nanoboxes with an Aqueous Etchant Based on $\mathrm{Fe}(\mathrm{NO} 3) 3$ or NH4OH. Nano Letters 7, 1764-1769 (2007). 
136. Jana, N.R., Gearheart, L. \& Murphy, C.J. Wet Chemical Synthesis of High Aspect Ratio Cylindrical Gold Nanorods. The Journal of Physical Chemistry B 105, 4065-4067 (2001).

137. Jana, N.R., Gearheart, L. \& Murphy, C.J. Wet chemical synthesis of silver nanorods and nanowires of controllable aspect ratio. Chemical Communications, 617-618 (2001).

138. Sun, Y. \& Xia, Y. Shape-Controlled Synthesis of Gold and Silver Nanoparticles. Science 298, 2176-2179 (2002).

139. Tao, A.R., Habas, S. \& Yang, P. Shape Control of Colloidal Metal Nanocrystals. Small 4, 310-325 (2008).

140. Fan, J.A. et al. Plasmonic Mode Engineering with Templated Self-Assembled Nanoclusters. Nano Letters 12, 5318-5324 (2012).

141. Ni, S., Klein, M.J.K., Spencer, N.D. \& Wolf, H. Cascaded Assembly of Complex Multiparticle Patterns. Langmuir 30, 90-95 (2014).

142. Adams, S.M., Campione, S., Capolino, F. \& Ragan, R. Directing Cluster Formation of $\mathrm{Au}$ Nanoparticles from Colloidal Solution. Langmuir 29, 42424251 (2013).

143. Onses, M.S., Liu, C.-C., Thode, C.J. \& Nealey, P.F. Highly Selective Immobilization of Au Nanoparticles onto Isolated and Dense Nanopatterns of Poly(2-vinyl pyridine) Brushes down to Single-Particle Resolution. Langmuir 28, 7299-7307 (2012).

144. Mendes, P.M. et al. Gold Nanoparticle Patterning of Silicon Wafers Using Chemical e-Beam Lithography. Langmuir 20, 3766-3768 (2004). 
145. Polte, J. et al. Mechanism of Gold Nanoparticle Formation in the Classical Citrate Synthesis Method Derived from Coupled In Situ XANES and SAXS Evaluation. Journal of the American Chemical Society 132, 1296-1301 (2010).

146. Pong, B.-K. et al. New Insights on the Nanoparticle Growth Mechanism in the Citrate Reduction of Gold(III) Salt: Formation of the $\mathrm{Au}$ Nanowire Intermediate and Its Nonlinear Optical Properties. The Journal of Physical Chemistry C 111, 6281-6287 (2007).

147. Khan, Z., Singh, T., Hussain, J.I. \& Hashmi, A.A. Au(III)-CTAB reduction by ascorbic acid: Preparation and characterization of gold nanoparticles. Colloids and Surfaces B: Biointerfaces 104, 11-17 (2013).

148. Sun, K., Qiu, J., Liu, J. \& Miao, Y. Preparation and characterization of gold nanoparticles using ascorbic acid as reducing agent in reverse micelles. $J$ Mater Sci 44, 754-758 (2009).

149. Bastus, N.G., Comenge, J. \& Puntes, V. Kinetically Controlled Seeded Growth Synthesis of Citrate-Stabilized Gold Nanoparticles of up to $200 \mathrm{~nm}$ : Size Focusing versus Ostwald Ripening. Langmuir 27, 11098-11105 (2011).

150. Sau, T., Pal, A., Jana, N.R., Wang, Z.L. \& Pal, T. Size Controlled Synthesis of Gold Nanoparticles using Photochemically Prepared Seed Particles. Journal of Nanoparticle Research 3, 257-261 (2001).

151. Zheng, Y. et al. Seed-Mediated Synthesis of Single-Crystal Gold Nanospheres with Controlled Diameters in the Range 5-30 nm and their Self-Assembly upon Dilution. Chemistry - An Asian Journal 8, $792-799$ (2013).

152. Jiali, N., Tao, Z. \& Zhongfan, L. One-step seed-mediated growth of 30-150 nm quasispherical gold nanoparticles with 2-mercaptosuccinic acid as a new reducing agent. Nanotechnology 18, 325607 (2007). 
153. Mallick, K., Wang, Z.L. \& Pal, T. Seed-mediated successive growth of gold particles accomplished by UV irradiation: a photochemical approach for sizecontrolled synthesis. Journal of Photochemistry and Photobiology A: Chemistry 140, 75-80 (2001).

154. Turkevich, J. Colloidal gold. Part I. Gold Bull 18, 86-91 (1985).

155. Turkevich, J., Stevenson, P.C. \& Hillier, J. A study of the nucleation and growth processes in the synthesis of colloidal gold. Discussions of the Faraday Society 11, 55-75 (1951).

156. Kimling, J. et al. Turkevich Method for Gold Nanoparticle Synthesis Revisited. The Journal of Physical Chemistry B 110, 15700-15707 (2006).

157. Ojea-Jimenez, I., Romero, F.M., Bastus, N.G. \& Puntes, V. Small Gold Nanoparticles Synthesized with Sodium Citrate and Heavy Water: Insights into the Reaction Mechanism. The Journal of Physical Chemistry C 114, 1800$1804(2010)$.

158. Nikoobakht, B. \& El-Sayed, M.A. Preparation and Growth Mechanism of Gold Nanorods (NRs) Using Seed-Mediated Growth Method. Chemistry of Materials 15, 1957-1962 (2003).

159. Jana, N.R., Gearheart, L. \& Murphy, C.J. Evidence for Seed-Mediated Nucleation in the Chemical Reduction of Gold Salts to Gold Nanoparticles. Chemistry of Materials 13, 2313-2322 (2001).

160. Jana, N.R., Gearheart, L. \& Murphy, C.J. Seed-Mediated Growth Approach for Shape-Controlled Synthesis of Spheroidal and Rod-like Gold Nanoparticles Using a Surfactant Template. Advanced Materials 13, 1389-1393 (2001). 
161. Johnson, C.J., Dujardin, E., Davis, S.A., Murphy, C.J. \& Mann, S. Growth and form of gold nanorods prepared by seed-mediated, surfactant-directed synthesis. Journal of Materials Chemistry 12, 1765-1770 (2002).

162. Gao, J., Bender, C.M. \& Murphy, C.J. Dependence of the Gold Nanorod Aspect Ratio on the Nature of the Directing Surfactant in Aqueous Solution. Langmuir 19, 9065-9070 (2003).

163. Huang, X., El-Sayed, I.H., Qian, W. \& El-Sayed, M.A. Cancer Cell Imaging and Photothermal Therapy in the Near-Infrared Region by Using Gold Nanorods. Journal of the American Chemical Society 128, 2115-2120 (2006).

164. Busbee, B.D., Obare, S.O. \& Murphy, C.J. An Improved Synthesis of HighAspect-Ratio Gold Nanorods. Advanced Materials 15, 414-416 (2003).

165. Jana, N.R. et al. Liquid crystalline assemblies of ordered gold nanorods. Journal of Materials Chemistry 12, 2909-2912 (2002).

166. Murphy, C.J. et al. The Many Faces of Gold Nanorods. The Journal of Physical Chemistry Letters 1, 2867-2875 (2010).

167. Sau, T.K. \& Murphy, C.J. Self-Assembly Patterns Formed upon Solvent Evaporation of Aqueous Cetyltrimethylammonium Bromide-Coated Gold Nanoparticles of Various Shapes. Langmuir 21, 2923-2929 (2005).

168. Lu, C.-L., Prasad, K.S., Wu, H.-L., Ho, J.-a.A. \& Huang, M.H. Au NanocubeDirected Fabrication of Au-Pd Core-Shell Nanocrystals with Tetrahexahedral, Concave Octahedral, and Octahedral Structures and Their Electrocatalytic Activity. Journal of the American Chemical Society 132, 14546-14553 (2010).

169. McLellan, J.M., Li, Z.-Y., Siekkinen, A.R. \& Xia, Y. The SERS Activity of a Supported Ag Nanocube Strongly Depends on Its Orientation Relative to Laser Polarization. Nano Letters 7, 1013-1017 (2007). 
170. Ringe, E. et al. Unraveling the Effects of Size, Composition, and Substrate on the Localized Surface Plasmon Resonance Frequencies of Gold and Silver Nanocubes: A Systematic Single-Particle Approach. The Journal of Physical Chemistry C 114, 12511-12516 (2010).

171. Zhang, J. et al. Concave Cubic Gold Nanocrystals with High-Index Facets. Journal of the American Chemical Society 132, 14012-14014 (2010).

172. Zhang, Q. et al. Seed-Mediated Synthesis of Ag Nanocubes with Controllable Edge Lengths in the Range of 30-200 nm and Comparison of Their Optical Properties. Journal of the American Chemical Society 132, 11372-11378 (2010).

173. Huang, Y., Ferhan, A.R., Gao, Y., Dandapat, A. \& Kim, D.-H. High-yield synthesis of triangular gold nanoplates with improved shape uniformity, tunable edge length and thickness. Nanoscale 6, 6496-6500 (2014).

174. Beeram, S.R. \& Zamborini, F.P. Purification of Gold Nanoplates Grown Directly on Surfaces for Enhanced Localized Surface Plasmon Resonance Biosensing. ACS Nano 4, 3633-3646 (2010).

175. Millstone, J.E., Hurst, S.J., Métraux, G.S., Cutler, J.I. \& Mirkin, C.A. Colloidal Gold and Silver Triangular Nanoprisms. Small 5, 646-664 (2009).

176. Xia, Y. et al. Gold Nanocages: From Synthesis to Theranostic Applications. Accounts of Chemical Research 44, 914-924 (2011).

177. Cheng, K. et al. Construction and Validation of Nano Gold Tripods for Molecular Imaging of Living Subjects. Journal of the American Chemical Society 136, 3560-3571 (2014).

178. Ali Umar, A. \& Oyama, M. High-Yield Synthesis of Tetrahedral-Like Gold Nanotripods Using an Aqueous Binary Mixture of Cetyltrimethylammonium 
Bromide and Hexamethylenetetramine. Crystal Growth \& Design 9, 11461152 (2009).

179. Chen, S., Wang, Z.L., Ballato, J., Foulger, S.H. \& Carroll, D.L. Monopod, Bipod, Tripod, and Tetrapod Gold Nanocrystals. Journal of the American Chemical Society 125, 16186-16187 (2003).

180. Hao, E., Bailey, R.C., Schatz, G.C., Hupp, J.T. \& Li, S. Synthesis and Optical Properties of "Branched" Gold Nanocrystals. Nano Letters 4, 327-330 (2004).

181. Hu, J. et al. Synthesis and Properties of Tadpole-Shaped Gold Nanoparticles. Journal of the American Chemical Society 126, 9470-9471 (2004).

182. Huang, T., Meng, F. \& Qi, L. Controlled Synthesis of Dendritic Gold Nanostructures Assisted by Supramolecular Complexes of Surfactant with Cyclodextrin. Langmuir 26, $7582-7589$ (2010).

183. Huang, D., Bai, X. \& Zheng, L. Ultrafast Preparation of Three-Dimensional Dendritic Gold Nanostructures in Aqueous Solution and Their Applications in Catalysis and SERS. The Journal of Physical Chemistry C 115, 14641-14647 (2011).

184. Xie, S. et al. Fast Growth Synthesis of Silver Dendrite Crystals Assisted by Sulfate Ion and Its Application for Surface-Enhanced Raman Scattering. The Journal of Physical Chemistry C 115, 9943-9951 (2011).

185. Lu, Y., Liu, G.L., Kim, J., Mejia, Y.X. \& Lee, L.P. Nanophotonic Crescent Moon Structures with Sharp Edge for Ultrasensitive Biomolecular Detection by Local Electromagnetic Field Enhancement Effect. Nano Letters 5, 119-124 (2005).

186. Bukasov, R. \& Shumaker-Parry, J.S. Highly Tunable Infrared Extinction Properties of Gold Nanocrescents. Nano Letters 7, 1113-1118 (2007). 
187. Li, L., Wang, Z., Huang, T., Xie, J. \& Qi, L. Porous Gold Nanobelts Templated by Metal,àíSurfactant Complex Nanobelts. Langmuir 26, 1233012335 (2010).

188. Oren, R. et al. Organization of Nanoparticles in Polymer Brushes. Journal of the American Chemical Society 131, 1670-1671 (2009).

189. Vaia, R.A. \& Maguire, J.F. Polymer Nanocomposites with Prescribed Morphology: Going beyond Nanoparticle-Filled Polymers. Chemistry of Materials 19, 2736-2751 (2007).

190. Choi, W.S., Koo, H.Y., Kim, J.Y. \& Huck, W.T.S. Collective Behavior of Magnetic Nanoparticles in Polyelectrolyte Brushes. Advanced Materials 20, 4504-4508 (2008).

191. Gupta, S. et al. Poly(2-(dimethylamino)ethyl methacrylate) Brushes with Incorporated Nanoparticles as a SERS Active Sensing Layer. Advanced Functional Materials 20, 1756-1761 (2010).

192. Snaith, H.J. et al. Self-Organization of Nanocrystals in Polymer Brushes. Application in Heterojunction Photovoltaic Diodes. Nano Letters 5, 1653-1657 (2005).

193. Tokareva, I., Minko, S., Fendler, J.H. \& Hutter, E. Nanosensors Based on Responsive Polymer Brushes and Gold Nanoparticle Enhanced Transmission Surface Plasmon Resonance Spectroscopy. Journal of the American Chemical Society 126, 15950-15951 (2004).

194. Dominguez, M.M., Wathier, M., Grinstaff, M.W. \& Schaus, S.E. Immobilized Hydrogels for Screening of Molecular Interactions. Analytical Chemistry 79, 1064-1066 (2007). 
195. Ellington, A.A., Kullo, I.J., Bailey, K.R. \& Klee, G.G. Antibody-Based Protein Multiplex Platforms: Technical and Operational Challenges. Clinical Chemistry 56, 186-193 (2010).

196. Kusnezow, W. \& Hoheisel, J.D. Solid supports for microarray immunoassays. Journal of Molecular Recognition 16, 165-176 (2003).

197. Lynch, M. et al. Functional protein nanoarrays for biomarker profiling. Proteomics 4, 1695-1702 (2004).

198. MacBeath, G. Protein microarrays and proteomics. Nature Genetics 32, 526$532(2002)$.

199. Sun, Y.S. et al. Macromolecular Scaffolds for Immobilizing Small Molecule Microarrays in Label-Free Detection of Protein-Ligand Interactions on Solid Support. Analytical Chemistry 81, 5373-5380 (2009).

200. Templin, M.F. et al. Protein microarrays: Promising tools for proteomic research. Proteomics 3, 2155-2166 (2003).

201. Hucknall, A., Rangarajan, S. \& Chilkoti, A. In Pursuit of Zero: Polymer Brushes that Resist the Adsorption of Proteins. Advanced Materials 21, 2441$2446(2009)$.

202. Masson, J.-F. et al. Biocompatible polymers for antibody support on gold surfaces. Talanta 67, 918-925 (2005).

203. Carignano, M.A. \& Szleifer, I. Prevention of protein adsorption by flexible and rigid chain molecules. Colloids and Surfaces B: Biointerfaces 18, 169-182 (2000).

204. Charles, P. et al. Reduction of Non-Specific Protein Adsorption Using Poly(ethylene) Glycol (PEG) Modified Polyacrylate Hydrogels In 
Immunoassays for Staphylococcal Enterotoxin B Detection. Sensors 9, 645655 (2009).

205. Jeyachandran, Y.L., Mielczarski, J.A., Mielczarski, E. \& Rai, B. Efficiency of blocking of non-specific interaction of different proteins by BSA adsorbed on hydrophobic and hydrophilic surfaces. Journal of Colloid and Interface Science 341, 136-142 (2010).

206. Volden, S., Zhu, K., Nystrom, B. \& Glomm, W.R. Use of cellulose derivatives on gold surfaces for reduced nonspecific adsorption of immunoglobulin G. Colloids and Surfaces B: Biointerfaces 72, 266-271 (2009).

207. Rusmini, F., Zhong, Z. \& Feijen, J. Protein Immobilization Strategies for Protein Biochips. Biomacromolecules 8, 1775-1789 (2007).

208. Cullen, S.P., Liu, X., Mandel, I.C., Himpsel, F.J. \& Gopalan, P. Polymeric Brushes as Functional Templates for Immobilizing Ribonuclease A:, Äâ Study of Binding Kinetics and Activity. Langmuir 24, 913-920 (2007).

209. Dong, R., Krishnan, S., Baird, B.A., Lindau, M. \& Ober, C.K. Patterned Biofunctional Poly(acrylic acid) Brushes on Silicon Surfaces. Biomacromolecules 8, 3082-3092 (2007).

210. Trmcic-Cvitas, J. et al. Biofunctionalized Protein Resistant Oligo(ethylene glycol)-Derived Polymer Brushes as Selective Immobilization and Sensing Platforms. Biomacromolecules 10, 2885-2894 (2009).

211. Dai, J. et al. High-Capacity Binding of Proteins by Poly(Acrylic Acid) Brushes and Their Derivatives. Langmuir 22, 4274-4281 (2006).

212. Kusumo, A. et al. High Capacity, Charge-Selective Protein Uptake by Polyelectrolyte Brushes. Langmuir 23, 4448-4454 (2007). 
213. Gautrot, J.E., Huck, W.T.S., Welch, M. \& Ramstedt, M. Protein-Resistant NTA-Functionalized Polymer Brushes for Selective and Stable Immobilization of Histidine-Tagged Proteins. ACS Applied Materials \& Interfaces 2, 193-202 (2009).

214. Jain, P., Dai, J., Baker, G.L. \& Bruening, M.L. Rapid Synthesis of Functional Polymer Brushes by Surface-Initiated Atom Transfer Radical Polymerization of an Acidic Monomer. Macromolecules 41, 8413-8417 (2008).

215. Salgado, E.N., Radford, R.J. \& Tezcan, F.A. Metal-Directed Protein SelfAssembly. Accounts of Chemical Research 43, 661-672 (2010).

216. Brown, A.A., Khan, N.S., Steinbock, L. \& Huck, W.T.S. Synthesis of oligo(ethylene glycol) methacrylate polymer brushes. European Polymer Journal 41, 1757-1765 (2005).

217. Feng, W., Zhu, S., Ishihara, K. \& Brash, J.L. Protein resistant surfaces: Comparison of acrylate graft polymers bearing oligo-ethylene oxide and phosphorylcholine side chains. Biointerphases 1, 50-60 (2006).

218. Ferhan, A.R. \& Kim, D.-H. In-stacking: a strategy for 3D nanoparticle assembly in densely-grafted polymer brushes. Journal of Materials Chemistry 22, 1274-1277 (2012).

219. Jain, P., Baker, G.L. \& Bruening, M.L. Applications of Polymer Brushes in Protein Analysis and Purification. Annual Review of Analytical Chemistry 2, 387-408 (2009).

220. Jin, Z., Feng, W., Zhu, S., Sheardown, H. \& Brash, J.L. Protein-resistant polyurethane via surface-initiated atom transfer radical polymerization of oligo(ethylene glycol) methacrylate. Journal of Biomedical Materials Research Part A 91A, 1189-1201 (2009). 
221. Kim, E. et al. Fabrication of nonbiofouling surface and its application to surface plasmon field-enhanced fluorescence spectroscopy. BioChip J. 2, 103$110(2008)$.

222. Kim, Y.-P. et al. Activity-Based Assay of Matrix Metalloproteinase on Nonbiofouling Surfaces Using Time-of-Flight Secondary Ion Mass Spectrometry. Analytical Chemistry 80, 5094-5102 (2008).

223. Krishnan, S., Weinman, C.J. \& Ober, C.K. Advances in polymers for antibiofouling surfaces. Journal of Materials Chemistry 18, 3405-3413 (2008).

224. Lee, B.S., Chi, Y.S., Lee, K.-B., Kim, Y.-G. \& Choi, I.S. Functionalization of Poly(oligo(ethylene glycol) methacrylate) Films on Gold and $\mathrm{Si} / \mathrm{SiO} 2$ for Immobilization of Proteins and Cells: SPR and QCM Studies. Biomacromolecules 8, 3922-3929 (2007).

225. Ma, H., Hyun, J., Stiller, P. \& Chilkoti, A. "Non-Fouling" Oligo(ethylene glycol)- Functionalized Polymer Brushes Synthesized by Surface-Initiated Atom Transfer Radical Polymerization. Advanced Materials 16, 338-341 (2004).

226. Ma, H., Li, D., Sheng, X., Zhao, B. \& Chilkoti, A. Protein-Resistant Polymer Coatings on Silicon Oxide by Surface-Initiated Atom Transfer Radical Polymerization. Langmuir 22, 3751-3756 (2006).

227. Hucknall, A. et al. Simple Fabrication of Antibody Microarrays on Nonfouling Polymer Brushes with Femtomolar Sensitivity for Protein Analytes in Serum and Blood. Advanced Materials 21, 1968-1971 (2009).

228. Efremova, N.V., Sheth, S.R. \& Leckband, D.E. Protein-Induced Changes in Poly(ethylene glycol) Brushes: Molecular Weight and Temperature Dependence. Langmuir 17, 7628-7636 (2001). 
229. Sheth, S.R. \& Leckband, D. Measurements of attractive forces between proteins and end-grafted poly(ethylene glycol) chains. Proceedings of the National Academy of Sciences 94, 8399-8404 (1997).

230. Unsworth, L.D., Sheardown, H. \& Brash, J.L. Protein-Resistant Poly(ethylene oxide)-Grafted Surfaces: Chain Density-Dependent Multiple Mechanisms of Action. Langmuir 24, 1924-1929 (2008).

231. Hu, W., Liu, Y., Lu, Z. \& Li, C.M. Poly[oligo(ethylene glycol) methacrylateco-glycidyl methacrylate] Brush Substrate for Sensitive Surface Plasmon Resonance Imaging Protein Arrays. Advanced Functional Materials 20, 34973503 (2010).

232. Akkahat, P. \& Hoven, V.P. Introducing surface-tethered poly(acrylic acid) brushes as 3D functional thin film for biosensing applications. Colloids and Surfaces B: Biointerfaces 86, 198-205 (2011).

233. Barbey, R., Kauffmann, E., Ehrat, M. \& Klok, H.-A. Protein Microarrays Based on Polymer Brushes Prepared via Surface-Initiated Atom Transfer Radical Polymerization. Biomacromolecules 11, 3467-3479 (2010).

234. Fang, F., Satulovsky, J. \& Szleifer, I. Kinetics of Protein Adsorption and Desorption on Surfaces with Grafted Polymers. Biophysical Journal 89, 1516$1533(2005)$.

235. Kim, J.U. \& Matsen, M.W. Repulsion Exerted on a Spherical Particle by a Polymer Brush. Macromolecules 41, 246-252 (2007).

236. Xue, C., Choi, B.-C., Choi, S., Braun, P.V. \& Leckband, D.E. Protein Adsorption Modes Determine Reversible Cell Attachment on Poly(N-isopropyl acrylamide) Brushes. Advanced Functional Materials 22, 2394-2401 (2012). 
237. Kim, J.U. \& O'Shaughnessy, B. Nanoinclusions in Dry Polymer Brushes. Macromolecules 39, 413-425 (2005).

238. Lee, K.-B., Park, S.-J., Mirkin, C.A., Smith, J.C. \& Mrksich, M. Protein Nanoarrays Generated By Dip-Pen Nanolithography. Science 295, 1702-1705 (2002).

239. Murata, M., Arakawa, M., Yoshida, T. \& Hato, M. Binding of immunoglobulin molecules to preadsorbed protein A layers as observed by surface forces measurements. Colloids and Surfaces B: Biointerfaces 12, 35-47 (1998).

240. Silverton, E.W., Navia, M.A. \& Davies, D.R. Three-dimensional structure of an intact human immunoglobulin. Proceedings of the National Academy of Sciences 74, 5140-5144 (1977).

241. Jossang, T., Feder, J. \& Rosenqvist, E. Photon correlation spectroscopy of human IgG. J Protein Chem 7, 165-171 (1988).

242. Chen, Y.-Y. et al. Colorimetric Assay for Lead Ions Based on the Leaching of Gold Nanoparticles. Analytical Chemistry 81, 9433-9439 (2009).

243. Lee, H.Y. et al. A Selective Fluoroionophore Based on BODIPYfunctionalized Magnetic Silica Nanoparticles: Removal of $\mathrm{Pb} 2+$ from Human Blood. Angewandte Chemie International Edition 48, 1239-1243 (2009).

244. Liu, C.-W., Hsieh, Y.-T., Huang, C.-C., Lin, Z.-H. \& Chang, H.-T. Detection of mercury(ii) based on $\mathrm{Hg} 2+-\mathrm{DNA}$ complexes inducing the aggregation of gold nanoparticles. Chemical Communications, 2242-2244 (2008).

245. Si, P., Kannan, P., Guo, L., Son, H. \& Kim, D.-H. Highly stable and sensitive glucose biosensor based on covalently assembled high density $\mathrm{Au}$ nanostructures. Biosensors and Bioelectronics 26, 3845-3851 (2011). 
246. Wilson, R. The use of gold nanoparticles in diagnostics and detection. Chemical Society Reviews 37, 2028-2045 (2008).

247. Yan, J. \& Indra, E.M. Colorimetric Method for Determining Pb2+ Ions in Water Enhanced with Non-Precious-Metal Nanoparticles. Analytical Chemistry 84, 6122-6127 (2012).

248. Mazumdar, D., Liu, J., Lu, G., Zhou, J. \& Lu, Y. Easy-to-use dipstick tests for detection of lead in paints using non-cross-linked gold nanoparticle-DNAzyme conjugates. Chemical Communications 46, 1416-1418 (2010).

249. Bonk, S.M. \& Lisdat, F. Layer-by-layer assembly of electro-active gold nanoparticle/cytochrome c multilayers. Biosensors and Bioelectronics 25, 739744 (2009).

250. Ferhan, A.R., Guo, L. \& Kim, D.-H. Influence of Ionic Strength and Surfactant Concentration on Electrostatic Surfacial Assembly of Cetyltrimethylammonium Bromide-Capped Gold Nanorods on Fully Immersed Glass. Langmuir 26, 12433-12442 (2010).

251. Morel, A.-L. et al. Optimized immobilization of gold nanoparticles on planar surfaces through alkyldithiols and their use to build 3D biosensors. Colloids and Surfaces B: Biointerfaces 81, 304-312 (2010).

252. Zhou, F., Yuan, L., Wang, H., Li, D. \& Chen, H. Gold Nanoparticle Layer: A Promising Platform for Ultra-Sensitive Cancer Detection. Langmuir 27, 2155$2158(2011)$.

253. Diamanti, S., Arifuzzaman, S., Genzer, J. \& Vaia, R.A. Tuning Gold Nanoparticle,àíPoly(2-hydroxyethyl methacrylate) Brush Interactions: From Reversible Swelling to Capture and Release. ACS Nano 3, 807-818 (2009). 
254. Hossain, S.M.Z. \& Brennan, J.D. Beta-Galactosidase-Based Colorimetric Paper Sensor for Determination of Heavy Metals. Analytical Chemistry 83, 8772-8778 (2011).

255. Lee, Y.-F. \& Huang, C.-C. Colorimetric Assay of Lead Ions in Biological Samples Using a Nanogold-Based Membrane. ACS Applied Materials \& Interfaces 3, 2747-2754 (2011).

256. Mentele, M.M., Cunningham, J., Koehler, K., Volckens, J. \& Henry, C.S. Microfluidic Paper-Based Analytical Device for Particulate Metals. Analytical Chemistry 84, 4474-4480 (2012).

257. Guo, L., Chen, G. \& Kim, D.-H. Three-Dimensionally Assembled Gold Nanostructures for Plasmonic Biosensors. Analytical Chemistry 82, 5147-5153 (2010).

258. Bridgewater, B.M. \& Parkin, G. Lead Poisoning and the Inactivation of 5Aminolevulinate Dehydratase as Modeled by the Tris(2-mercapto-1phenylimidazolyl)hydroborato Lead Complex, $\{[\mathrm{TmPh}] \mathrm{Pb}\}[\mathrm{ClO} 4]$. Journal of the American Chemical Society 122, 7140-7141 (2000).

259. Hirayama, T. et al. Colorimetric response to mercury-induced abstraction of triethylene glycol ligands from a gold nanoparticle surface. Dalton Transactions, 4705-4707 (2008).

260. Tian, M. \& Ihmels, H. Selective Colorimetric Detection of $\mathrm{Hg} 2+$ and $\mathrm{Mg} 2+$ with Crown Ether Substituted N-Aryl-9-aminobenzo[b]quinolizinium Derivatives. European Journal of Organic Chemistry 2011, 4145-4153 (2011).

261. Bi, Z., Chapman, C.S., Salaün, P. \& van den Berg, C.M.G. Determination of Lead and Cadmium in Sea- and Freshwater by Anodic Stripping Voltammetry with a Vibrating Bismuth Electrode. Electroanalysis 22, 2897-2907 (2010). 
262. Fu, X. et al. "Turn-on" Fluorescence Detection of Lead Ions Based on Accelerated Leaching of Gold Nanoparticles on the Surface of Graphene. ACS Applied Materials \& Interfaces 4, 1080-1086 (2012).

263. Guo, J., Chai, Y., Yuan, R., Song, Z. \& Zou, Z. Lead (II) carbon paste electrode based on derivatized multi-walled carbon nanotubes: Application to lead content determination in environmental samples. Sensors and Actuators B: Chemical 155, 639-645 (2011).

264. Huang, K.-W., Yu, C.-J. \& Tseng, W.-L. Sensitivity enhancement in the colorimetric detection of lead(II) ion using gallic acid-capped gold nanoparticles: Improving size distribution and minimizing interparticle repulsion. Biosensors and Bioelectronics 25, $984-989$ (2010).

265. Liu, Y.-C., Chiang, C.-K., Chang, H.-T., Lee, Y.-F. \& Huang, C.-C. Using a Functional Nanogold Membrane Coupled with Laser Desorption/Ionization Mass Spectrometry to Detect Lead Ions in Biofluids. Advanced Functional Materials 21, 4448-4455 (2011).

266. Nagraj, N., Liu, J., Sterling, S., Wu, J. \& Lu, Y. DNAzyme catalytic beacon sensors that resist temperature-dependent variations. Chemical Communications, 4103-4105 (2009).

267. Ngoc Bui, M.-P., Li, C.A., Han, K.N., Pham, X.-H. \& Seong, G.H. Simultaneous detection of ultratrace lead and copper with gold nanoparticles patterned on carbon nanotube thin film. Analyst 137, 1888-1894 (2012).

268. Pan, D., Wang, Y., Chen, Z., Lou, T. \& Qin, W. Nanomaterial/IonophoreBased Electrode for Anodic Stripping Voltammetric Determination of Lead: An Electrochemical Sensing Platform toward Heavy Metals. Analytical Chemistry 81, 5088-5094 (2009). 
269. Saadeh, S.M. et al. A new potentiometric thiosalicylamide-functionalized polysiloxane carbon paste electrode for lead(II) determination. Journal of Electroanalytical Chemistry 687, 11-17 (2012).

270. Shete, V.S. \& Benson, D.E. Protein Design Provides Lead(II) Ion Biosensors for Imaging Molecular Fluxes around Red Blood Cells. Biochemistry 48, 462$470(2008)$.

271. Sudibya, H.G., He, Q., Zhang, H. \& Chen, P. Electrical Detection of Metal Ions Using Field-Effect Transistors Based on Micropatterned Reduced Graphene Oxide Films. ACS Nano 5, 1990-1994 (2011).

272. Wang, H. et al. Engineering a Unimolecular DNA-Catalytic Probe for Single Lead Ion Monitoring. Journal of the American Chemical Society 131, 82218226 (2009).

273. Wang, Z., Lee, J.H. \& Lu, Y. Label-Free Colorimetric Detection of Lead Ions with a Nanomolar Detection Limit and Tunable Dynamic Range by using Gold Nanoparticles and DNAzyme. Advanced Materials 20, 3263-3267 (2008).

274. Xiang, Y., Tong, A. \& Lu, Y. Abasic Site-Containing DNAzyme and Aptamer for Label-Free Fluorescent Detection of $\mathrm{Pb} 2+$ and Adenosine with High Sensitivity, Selectivity, and Tunable Dynamic Range. Journal of the American Chemical Society 131, 15352-15357 (2009).

275. Yantasee, W. et al. Direct detection of $\mathrm{Pb}$ in urine and $\mathrm{Cd}, \mathrm{Pb}, \mathrm{Cu}$, and $\mathrm{Ag}$ in natural waters using electrochemical sensors immobilized with DMSA functionalized magnetic nanoparticles. Analyst 133, 348-355 (2008).

276. Weigl, B.H. \& Wolfbeis, O.S. Capillary Optical Sensors. Analytical Chemistry 66, 3323-3327 (1994). 
277. Gupta, S., Agrawal, M., Uhlmann, P., Simon, F. \& Stamm, M. Poly(Nisopropyl acrylamide),àíGold Nanoassemblies on Macroscopic Surfaces: Fabrication, Characterization, and Application. Chemistry of Materials 22, 504-509 (2009).

278. Azzaroni, O. et al. Synthesis of gold nanoparticles inside polyelectrolyte brushes. Journal of Materials Chemistry 17, 3433-3439 (2007).

279. Liu, J. \& Lu, Y. Preparation of aptamer-linked gold nanoparticle purple aggregates for colorimetric sensing of analytes. Nat. Protocols 1, 246-252 (2006).

280. Feng, W., Chen, R., Brash, J.L. \& Zhu, S. Surface-Initiated Atom Transfer Radical Polymerization of Oligo(ethylene glycol) Methacrylate: Effect of Solvent on Graft Density. Macromolecular Rapid Communications 26, 1383$1388(2005)$.

281. Zhao, B. \& Brittain, W.J. Polymer brushes: surface-immobilized macromolecules. Progress in Polymer Science 25, 677-710 (2000).

282. Bhat, R.R. \& Genzer, J. Combinatorial study of nanoparticle dispersion in surface-grafted macromolecular gradients. Applied Surface Science 252, 25492554 (2006).

283. Bunka, D.H.J. \& Stockley, P.G. Aptamers come of age: at last. Nat Rev Micro 4, 588-596 (2006).

284. Liu, J., Cao, Z. \& Lu, Y. Functional Nucleic Acid Sensors. Chemical Reviews 109, 1948-1998 (2009).

285. Cho, H. et al. Aptamer-Based SERRS Sensor for Thrombin Detection. Nano Letters 8, 4386-4390 (2008). 
286. Das, J. et al. An ultrasensitive universal detector based on neutralizer displacement. Nat Chem 4, 642-648 (2012).

287. Wang, G., Wang, Y., Chen, L. \& Choo, J. Nanomaterial-assisted aptamers for optical sensing. Biosensors and Bioelectronics 25, 1859-1868 (2010).

288. Wei, H., Li, B., Li, J., Wang, E. \& Dong, S. Simple and sensitive aptamerbased colorimetric sensing of protein using unmodified gold nanoparticle probes. Chemical Communications, 3735-3737 (2007).

289. $\mathrm{Xu}, \mathrm{H}$. et al. Label-free impedimetric thrombin sensor based on poly(pyrrolenitrilotriacetic acid)-aptamer film. Biosensors and Bioelectronics 41, 90-95 (2013).

290. Tennico, Y.H., Hutanu, D., Koesdjojo, M.T., Bartel, C.M. \& Remcho, V.T. On-Chip Aptamer-Based Sandwich Assay for Thrombin Detection Employing Magnetic Beads and Quantum Dots. Analytical Chemistry 82, 5591-5597 (2010).

291. Abcam Thrombin (Factor II) Human ELISA Kit (ab108909). retrieved, Aug 2015, from, http://www.abcam.com/thrombin-factor-ii-human-elisa-kitab108909.html.

292. Anaspec SensoLyte ${ }^{\circledR} 520$ Thrombin Activity Assay Kit *Fluorimetric*. retrieved, Aug 2015, from, http://www.anaspec.com/products/product.asp?id=48466.

293. Abnova Thrombin (Human) ELISA Kit. retrieved, Aug 2015, from, http://www.abnova.com/products/products detail.asp?catalog id=KA0511.

294. Zheng, Y., Yuan, Y., Chai, Y. \& Yuan, R. A label-free electrochemical aptasensor based on the catalysis of manganese porphyrins for detection of thrombin. Biosensors and Bioelectronics 66, 585-589 (2015). 
295. Wang, K. et al. A label-free aptasensor for highly sensitive detection of ATP and thrombin based on metal-enhanced PicoGreen fluorescence. Biosensors and Bioelectronics 63, 172-177 (2015).

296. Shankar, S.S., Rai, A., Ahmad, A. \& Sastry, M. Controlling the Optical Properties of Lemongrass Extract Synthesized Gold Nanotriangles and Potential Application in Infrared-Absorbing Optical Coatings. Chemistry of Materials 17, 566-572 (2005).

297. Tsuji, M., Hashimoto, M., Nishizawa, Y., Kubokawa, M. \& Tsuji, T. Microwave-Assisted Synthesis of Metallic Nanostructures in Solution. Chemistry - A European Journal 11, 440-452 (2005).

298. Wang, D. \& Imae, T. Watching the Transformation from Au Nanoparticles to Microplates. Chemistry Letters 35, 1152-1153 (2006).

299. Xiong, Y. et al. Corrosion-Based Synthesis of Single-Crystal Pd Nanoboxes and Nanocages and Their Surface Plasmon Properties. Angewandte Chemie International Edition 44, 7913-7917 (2005).

300. Nusz, G.J., Curry, A.C., Marinakos, S.M., Wax, A. \& Chilkoti, A. Rational Selection of Gold Nanorod Geometry for Label-Free Plasmonic Biosensors. ACS Nano 3, 795-806 (2009).

301. Darbha, G.K., Rai, U.S., Singh, A.K. \& Ray, P.C. Gold-Nanorod-Based Sensing of Sequence Specific HIV-1 Virus DNA by Using Hyper-Rayleigh Scattering Spectroscopy. Chemistry - A European Journal 14, 3896-3903 (2008).

302. Haes, A., Stuart, D., Nie, S. \& Van Duyne, R. Using Solution-Phase Nanoparticles, Surface-Confined Nanoparticle Arrays and Single 
Nanoparticles as Biological Sensing Platforms. Journal of Fluorescence 14, $355-367$ (2004).

303. Liu, X. et al. A One-Step Homogeneous Immunoassay for Cancer Biomarker Detection Using Gold Nanoparticle Probes Coupled with Dynamic Light Scattering. Journal of the American Chemical Society 130, 2780-2782 (2008).

304. Singh, A.K. et al. Gold Nanorod Based Selective Identification of Escherichia coli Bacteria Using Two-Photon Rayleigh Scattering Spectroscopy. ACS Nano 3, 1906-1912 (2009).

305. Kim, D.-K. et al. Label-Free DNA Biosensor Based on Localized Surface Plasmon Resonance Coupled with Interferometry. Analytical Chemistry 79, 1855-1864 (2007).

306. Nath, N. \& Chilkoti, A. A Colorimetric Gold Nanoparticle Sensor To Interrogate Biomolecular Interactions in Real Time on a Surface. Analytical Chemistry 74, 504-509 (2001).

307. Guerrero-Martínez, A., Pérez-Juste, J., Carbó-Argibay, E., Tardajos, G. \& LizMarzán, L.M. Gemini-Surfactant-Directed Self-Assembly of Monodisperse Gold Nanorods into Standing Superlattices. Angewandte Chemie International Edition 48, 9484-9488 (2009).

308. Kawamura, G., Yang, Y. \& Nogami, M. Facile assembling of gold nanorods with large aspect ratio and their surface-enhanced Raman scattering properties. Applied Physics Letters 90, - (2007).

309. Nakashima, H., Furukawa, K., Kashimura, Y. \& Torimitsu, K. Self-Assembly of Gold Nanorods Induced by Intermolecular Interactions of Surface-Anchored Lipids. Langmuir 24, 5654-5658 (2008). 
310. Zhang, X. \& Imae, T. Perpendicular Superlattice Growth of Hydrophobic Gold Nanorods on Patterned Silicon Substrates via Evaporation-Induced SelfAssembling. The Journal of Physical Chemistry C 113, 5947-5951 (2009).

311. Jana, N.R. Shape Effect in Nanoparticle Self-Assembly. Angewandte Chemie International Edition 43, 1536-1540 (2004).

312. Nikoobakht, B., Wang, Z.L. \& El-Sayed, M.A. Self-Assembly of Gold Nanorods. The Journal of Physical Chemistry B 104, 8635-8640 (2000).

313. Sethi, M., Joung, G. \& Knecht, M.R. Stability and Electrostatic Assembly of Au Nanorods for Use in Biological Assays. Langmuir 25, 317-325 (2008).

314. Niidome, Y., Takahashi, H., Urakawa, S., Nishioka, K. \& Yamada, S. Immobilization of Gold Nanorods on the Glass Substrate by the Electrostatic Interactions for Localized Plasmon Sensing. Chemistry Letters 33, 454-455 (2004).

315. Xu, Z.-C. et al. Fabrication of gold nanorod self-assemblies from rod and sphere mixtures via shape self-selective behavior. Chemical Physics Letters 432, 222-225 (2006).

316. Kim, T., Lee, C.-H., Joo, S.-W. \& Lee, K. Kinetics of gold nanoparticle aggregation: Experiments and modeling. Journal of Colloid and Interface Science 318, 238-243 (2008).

317. Derjaguin, B. \& Landau, L. Theory of the stability of strongly charged lyophobic sols and of the adhesion of strongly charged particles in solutions of electrolytes. Progress in Surface Science 43, 30-59 (1993).

318. Collins, K.D., Neilson, G.W. \& Enderby, J.E. Ions in water: Characterizing the forces that control chemical processes and biological structure. Biophysical Chemistry 128, 95-104 (2007). 
319. Howard, S.C., Atkin, R. \& Craig, V.S.J. Effect of electrolyte species on the adsorption of a cationic surfactant to silica: The common intersection point. Colloids and Surfaces A: Physicochemical and Engineering Aspects 347, 109113 (2009).

320. Delgado, A.V., Gonzalez-Caballero, F., Hunter, R.J., Koopal, L.K. \& Lyklema, J. in Pure and Applied Chemistry, Vol. 77 17532005).

321. Atkin, R., Craig, V.S.J., Wanless, E.J. \& Biggs, S. Mechanism of cationic surfactant adsorption at the solid/aqueous interface. Advances in Colloid and Interface Science 103, 219-304 (2003).

322. Atkin, R., Craig, V.S.J. \& Biggs, S. Adsorption Kinetics and Structural Arrangements of Cationic Surfactants on Silica Surfaces. Langmuir 16, 93749380 (2000).

323. Atkin, R., Craig, V.S.J., Wanless, E.J. \& Biggs, S. The influence of chain length and electrolyte on the adsorption kinetics of cationic surfactants at the silica, Äìqueous solution interface. Journal of Colloid and Interface Science 266, 236-244 (2003).

324. Mangold, M.A., Calame, M., Mayor, M. \& Holleitner, A.W. Resonant Photoconductance of Molecular Junctions Formed in Gold Nanoparticle Arrays. Journal of the American Chemical Society 133, 12185-12191 (2011).

325. Anker, J.N. et al. Biosensing with plasmonic nanosensors. Nat Mater 7, 442453 (2008).

326. de la Rica, R. \& Stevens, M.M. Plasmonic ELISA for the ultrasensitive detection of disease biomarkers with the naked eye. Nat Nano 7, 821-824 (2012). 
327. Rodriguez-Lorenzo, L., de la Rica, R., Ålvarez-Puebla, R.A., Liz-Marzan, L.M. \& Stevens, M.M. Plasmonic nanosensors with inverse sensitivity by means of enzyme-guided crystal growth. Nat Mater 11, 604-607 (2012).

328. Agarwal, A. et al. Targeted gold nanorod contrast agent for prostate cancer detection by photoacoustic imaging. Journal of Applied Physics 102, - (2007).

329. Eghtedari, M. et al. High Sensitivity of In Vivo Detection of Gold Nanorods Using a Laser Optoacoustic Imaging System. Nano Letters 7, 1914-1918 (2007).

330. Niidome, T. et al. In Vivo Monitoring of Intravenously Injected Gold Nanorods Using Near-Infrared Light. Small 4, 1001-1007 (2008).

331. Ke, H. et al. Bifunctional gold nanorod-loaded polymeric microcapsules for both contrast-enhanced ultrasound imaging and photothermal therapy. Journal of Materials Chemistry 21, 5561-5564 (2011).

332. Shao, X. et al. 125I-Labeled Gold Nanorods for Targeted Imaging of Inflammation. ACS Nano 5, 8967-8973 (2011).

333. Kuo, W.-S. et al. Gold Nanorods in Photodynamic Therapy, as Hyperthermia Agents, and in Near-Infrared Optical Imaging. Angewandte Chemie International Edition 49, 2711-2715 (2010).

334. Li, X., Takashima, M., Yuba, E., Harada, A. \& Kono, K. PEGylated PAMAM dendrimer,Äìdoxorubicin conjugate-hybridized gold nanorod for combined photothermal-chemotherapy. Biomaterials 35, 6576-6584 (2014).

335. Li, X., Takeda, K., Yuba, E., Harada, A. \& Kono, K. Preparation of PEGmodified PAMAM dendrimers having a gold nanorod core and their application to photothermal therapy. Journal of Materials Chemistry B 2, 4167-4176 (2014). 
336. Ferrier, R.C. et al. Gold Nanorod Linking to Control Plasmonic Properties in Solution and Polymer Nanocomposites. Langmuir 30, 1906-1914 (2014).

337. Funston, A.M., Novo, C., Davis, T.J. \& Mulvaney, P. Plasmon Coupling of Gold Nanorods at Short Distances and in Different Geometries. Nano Letters 9, 1651-1658 (2009).

338. Shao, L. et al. Angle- and Energy-Resolved Plasmon Coupling in Gold Nanorod Dimers. ACS Nano 4, 3053-3062 (2010).

339. Jana, N.R. \& Pal, T. Anisotropic Metal Nanoparticles for Use as SurfaceEnhanced Raman Substrates. Advanced Materials 19, 1761-1765 (2007).

340. Adams, S.M. et al. Non-lithographic SERS Substrates: Tailoring Surface Chemistry for Au Nanoparticle Cluster Assembly. Small 8, 2239-2249 (2012).

341. Vigderman, L., Khanal, B.P. \& Zubarev, E.R. Functional Gold Nanorods: Synthesis, Self-Assembly, and Sensing Applications. Advanced Materials 24, 4811-4841 (2012).

342. Hamon, C., Bizien, T., Artzner, F., Even-Hernandez, P. \& Marchi, V. Replacement of CTAB with peptidic ligands at the surface of gold nanorods and their self-assembling properties. Journal of Colloid and Interface Science 424, 90-97 (2014).

343. Nepal, D. et al. Control over Position, Orientation, and Spacing of Arrays of Gold Nanorods Using Chemically Nanopatterned Surfaces and Tailored Particle-Particle-Surface Interactions. ACS Nano 6, 5693-5701 (2012).

344. Lee, Y.H. et al. Using the Langmuir-Schaefer technique to fabricate large-area dense SERS-active Au nanoprism monolayer films. Nanoscale 5, 6404-6412 (2013). 
345. Ming, T. et al. Ordered Gold Nanostructure Assemblies Formed By Droplet Evaporation. Angewandte Chemie International Edition 47, 9685-9690 (2008).

346. Peng, B. et al. Vertically Aligned Gold Nanorod Monolayer on Arbitrary Substrates: Self-Assembly and Femtomolar Detection of Food Contaminants. ACS Nano 7, 5993-6000 (2013).

347. Peng, B. et al. Quantum dots on vertically aligned gold nanorod monolayer: plasmon enhanced fluorescence. Nanoscale 6, 5592-5598 (2014).

348. Qian, Y. et al. Flexible membranes of Ag-nanosheet-grafted polyamidenanofibers as effective 3D SERS substrates. Nanoscale 6, 4781-4788 (2014).

349. Tang, W., Chase, D.B. \& Rabolt, J.F. Immobilization of Gold Nanorods onto Electrospun Polycaprolactone Fibers Via Polyelectrolyte Decoration- A 3D SERS Substrate. Analytical Chemistry 85, 10702-10709 (2013).

350. Akin, M.S. et al. Large area uniform deposition of silver nanoparticles through bio-inspired polydopamine coating on silicon nanowire arrays for practical SERS applications. Journal of Materials Chemistry B 2, 4894-4900 (2014).

351. Zhang, Q., Lee, Y.H., Phang, I.Y., Lee, C.K. \& Ling, X.Y. Hierarchical 3D SERS Substrates Fabricated by Integrating Photolithographic Microstructures and Self-Assembly of Silver Nanoparticles. Small 10, 2703-2711 (2014).

352. Alvarez-Puebla, R.A. et al. Gold nanorods 3D-supercrystals as surface enhanced Raman scattering spectroscopy substrates for the rapid detection of scrambled prions. Proceedings of the National Academy of Sciences 108, 81578161 (2011).

353. Stewart, A.F. et al. Rational Design for the Controlled Aggregation of Gold Nanorods via Phospholipid Encapsulation for Enhanced Raman Scattering. ACS Nano 8, 5462-5467 (2014). 
354. Ferhan, A.R. et al. Solid-Phase Colorimetric Sensor Based on Gold Nanoparticle-Loaded Polymer Brushes: Lead Detection as a Case Study. Analytical Chemistry 85, 4094-4099 (2013).

355. Ebbesen, T.W., Lezec, H.J., Ghaemi, H.F., Thio, T. \& Wolff, P.A. Extraordinary optical transmission through sub-wavelength hole arrays. Nature 391, 667-669 (1998).

356. Gordon, R., Sinton, D., Kavanagh, K.L. \& Brolo, A.G. A New Generation of Sensors Based on Extraordinary Optical Transmission. Accounts of Chemical Research 41, 1049-1057 (2008).

357. Wang, Y. et al. Transmissive Nanohole Arrays for Massively-Parallel Optical Biosensing. ACS Photonics 1, 241-245 (2014).

358. Zhou, Z.-K. et al. Tuning Gold Nanorod-Nanoparticle Hybrids into Plasmonic Fano Resonance for Dramatically Enhanced Light Emission and Transmission. Nano Letters 11, 49-55 (2011).

359. Luk'yanchuk, B. et al. The Fano resonance in plasmonic nanostructures and metamaterials. Nat Mater 9, 707-715 (2010).

360. Tokman, M.D. \& Erukhimova, M.A. Anisotropy-Induced Transparency in Optically Dense Media. Radiophys Quantum El 57, 821-836 (2015).

361. Umadevi, S., Feng, X. \& Hegmann, T. Large Area Self-Assembly of Nematic Liquid-Crystal-Functionalized Gold Nanorods. Advanced Functional Materials 23, 1393-1403 (2013).

362. Kumar, J., Thomas, R., Swathi, R.S. \& Thomas, K.G. Au nanorod quartets and Raman signal enhancement: towards the design of plasmonic platforms. Nanoscale 6, 10454-10459 (2014). 
363. Farcau, C. \& Astilean, S. Evidence of a surface plasmon-mediated mechanism in the generation of the SERS background. Chemical Communications 47, 3861-3863 (2011).

364. Haynes, C.L. \& Van Duyne, R.P. Plasmon-Sampled Surface-Enhanced Raman Excitation Spectroscopy. The Journal of Physical Chemistry B 107, 7426-7433 (2003).

365. Cheng, Z.-Q. et al. Plasmonic nanorod arrays of a two-segment dimer and a coaxial cable with $1 \mathrm{~nm}$ gap for large field confinement and enhancement. Nanoscale 7, 1463-1470 (2015).

366. McFarland, A.D., Young, M.A., Dieringer, J.A. \& Van Duyne, R.P. Wavelength-Scanned Surface-Enhanced Raman Excitation Spectroscopy. The Journal of Physical Chemistry B 109, 11279-11285 (2005).

367. Ameer, F.S., Zhou, Y., Zou, S. \& Zhang, D. Wavelength-Dependent Correlations between Ultraviolet-Visible Intensities and Surface Enhanced Raman Spectroscopic Enhancement Factors of Aggregated Gold and Silver Nanoparticles. The Journal of Physical Chemistry C 118, 22234-22242 (2014).

368. Wustholz, K.L. et al. Structure-Activity Relationships in Gold Nanoparticle Dimers and Trimers for Surface-Enhanced Raman Spectroscopy. Journal of the American Chemical Society 132, 10903-10910 (2010).

369. Michaels, A.M., Nirmal, M. \& Brus, L.E. Surface Enhanced Raman Spectroscopy of Individual Rhodamine 6G Molecules on Large Ag Nanocrystals. Journal of the American Chemical Society 121, 9932-9939 (1999). 
370. Le Ru, E.C., Galloway, C. \& Etchegoin, P.G. On the connection between optical absorption/extinction and SERS enhancements. Physical Chemistry Chemical Physics 8, 3083-3087 (2006).

371. Chen, J. et al. Two-Dimensional Ag Nanoparticle Tetramer Array for SurfaceEnhanced Raman Scattering Measurements. The Journal of Physical Chemistry C 118, 22702-22710 (2014).

372. Gao, T. et al. Controlled Synthesis of Homogeneous Ag Nanosheet-Assembled Film for Effective SERS Substrate. ACS Applied Materials \& Interfaces 5, 7308-7314 (2013).

373. He, L. et al. Silver nanosheet-coated inverse opal film as a highly active and uniform SERS substrate. Journal of Materials Chemistry 22, 1370-1374 (2012).

374. Zhou, Y. et al. Graphene-silver nanohybrids for ultrasensitive surface enhanced Raman spectroscopy: size dependence of silver nanoparticles. Journal of Materials Chemistry C 2, 6850-6858 (2014). 


\section{List of Publications}

*Publications in this thesis are in bold

1. A Facile Method Towards Rough Morphology Polymer Brush for Increased Mobility of Embedded Nanoparticles Ferhan, AR.; Zainol, N.; Kim, DH. Polymer. 2015, 75, 57-63.

2. High-Yield Synthesis of Triangular Gold Nanoplates With Improved Shape Uniformity, Tunable Edge Length and Thickness

Huang, Y.; Ferhan, AR.; Gao, Y.; Dandapat, A.; Kim, DH. Nanoscale. 2014, 6, 6496-6500.

3. In Situ Synthesis of Protein-Resistant Poly(Oligo(Ethyelene Glycol) Methacrylate) Films in Capillary for Protein Separation Huang, R.; Ferhan, AR.; Guo, L.; Qiu, B.; Lin, Z.; Kim, DH.; Chen, G. RSC Advances. 2014, 4, 4883-4888.

4. Oriented Gold Nanoparticle Aggregation for Colorimetric Sensors with Surprisingly High Analytical Figures of Merit Guo, LH.; Xu, Y.; Ferhan, AR.; Chen, GN.; Kim, DH. Journal of the American Chemical Society. 2013, 135, 12338-12345.

5. Solid-Phase Colorimetric Sensor Based on Gold Nanoparticle-Loaded Polymer Brushes: Lead Detection as a Case Study Ferhan, AR.; Guo, LH.; Zhou, X.; Chen, P.; Hong, S.; Kim, DH. Analytical Chemistry. 2013, 85, 4094-4099.

6. Physical Immobilization of Antibodies in Densely-Grafted Polymer Brushes via Spot-Drying: Towards Optimal Protein Loading Ferhan, AR.; Kim, DH. RSC Advances. 2013, 3, 9785-9793.

7. Distance-Mediated Plasmonic Dimers for Reusable Colorimetric Switches: A Measurable Peak Shift of More than $60 \mathrm{~nm}$

Guo, LH.; Ferhan, AR.; Kim, DH. Small. 2013, 9, 234-240.

8. Tunable Scattered Colors Over a Wide Spectrum from a Single Nanoparticle Huang, YJ.; Ferhan, AR.; Kim, DH. Nanoscale. 2013, 5, 7772-7775.

9. In-Stacking: A Strategy for 3D Nanoparticle Assembly in DenselyGrafted Polymer Brushes

Ferhan, AR.; Kim, DH. Journal of Materials Chemistry. 2012, 22, 12741277.

10. Multilayered Polypyrrole-Coated Carbon Nanotubes To Improve Functional Stability and Electrical Properties of Neural Electrodes

Chen, HL.; Guo, LH.; Ferhan, AR.; Kim, DH. Journal of Physical Chemistry C. 2011, 115, 5492-5499. 
11. Nanoarray-Based Biomolecular Detection Using Individual Au Nanoparticles with Minimized Localized Surface Plasmon Resonance Variations

Guo, LH.; Ferhan, AR.; Lee, K.; Kim, DH. Analytical Chemistry. 2011, 83, 2605-2612.

12. Influence of Ionic Strength and Surfactant Concentration on Electrostatic Surfacial Assembly of Cetyltrimethylammonium BromideCapped Gold Nanorods on Fully-Immersed Glass

Ferhan, AR.; Guo, LH.; Kim, DH. Langmuir. 2010, 26, 12433-12422. 


\section{APPENDIX}

\section{A. List of Figures}

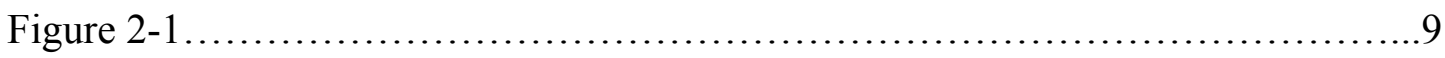

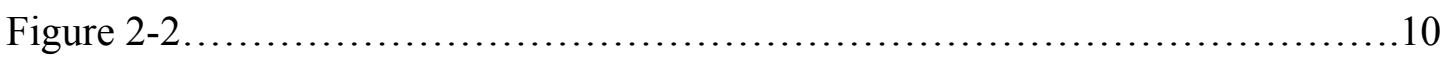

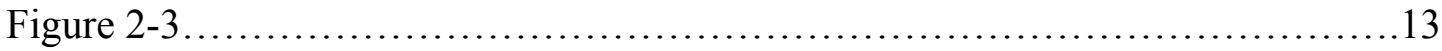

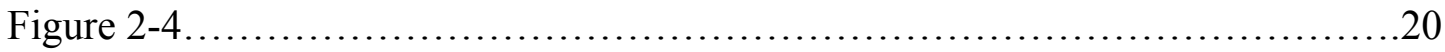

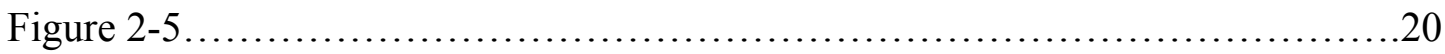

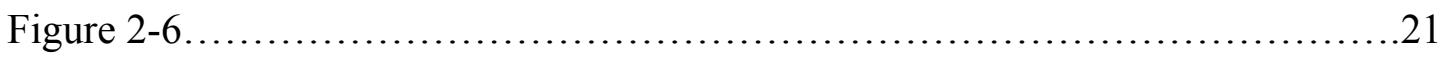

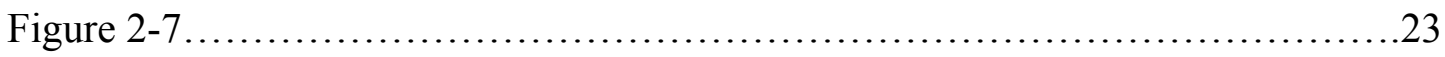

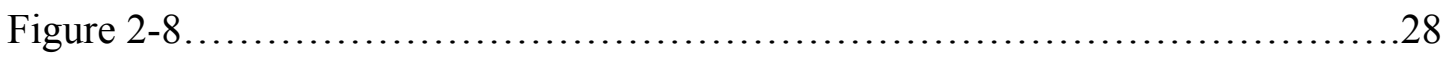

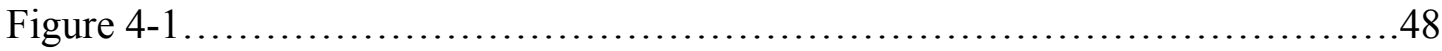

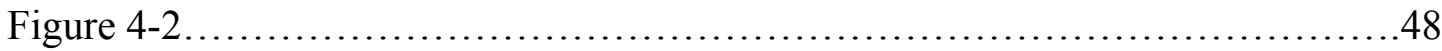

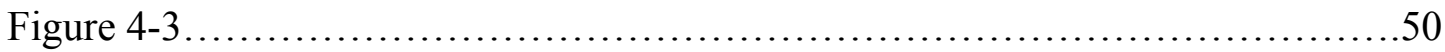

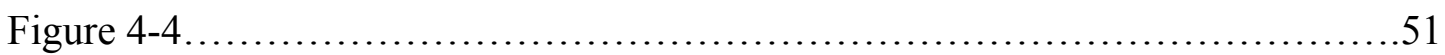

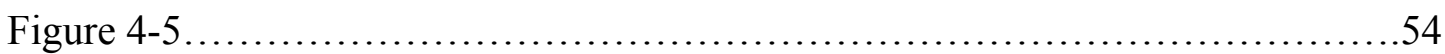

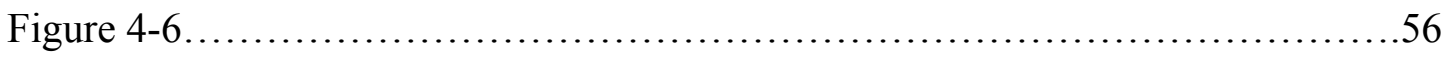

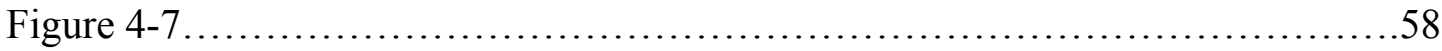

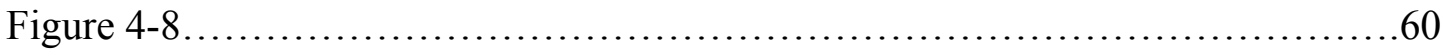

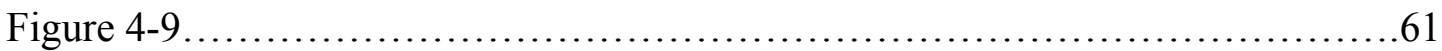

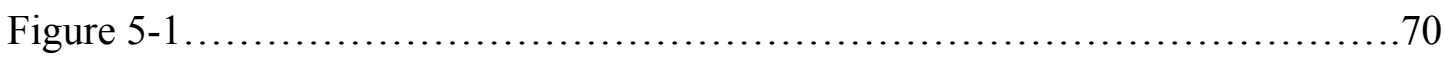

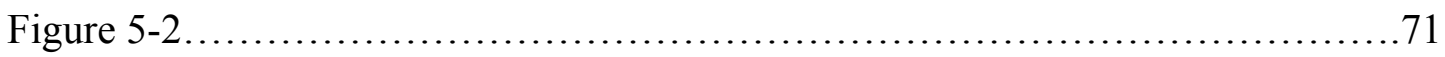

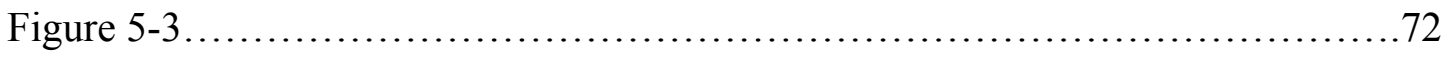

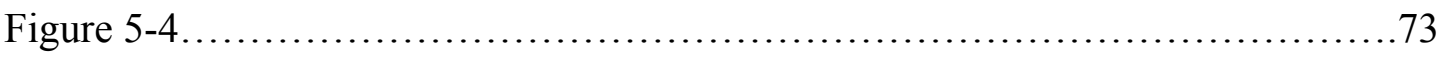

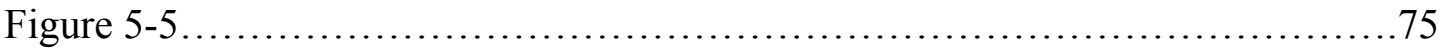

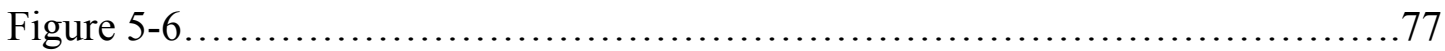

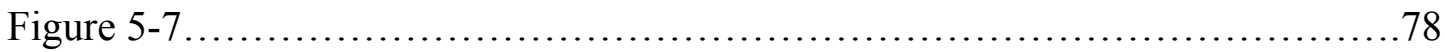

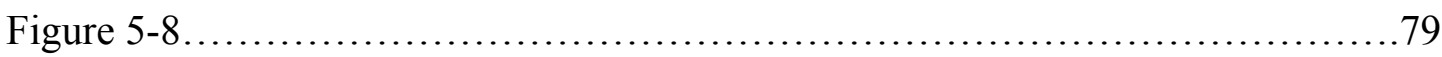

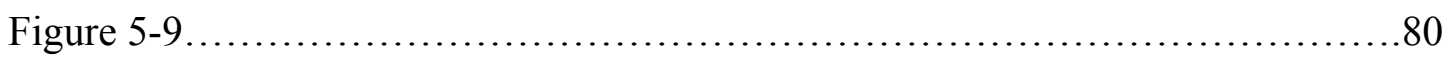




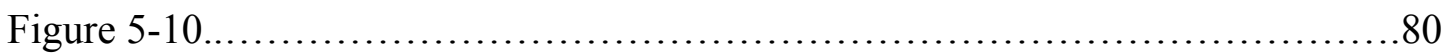

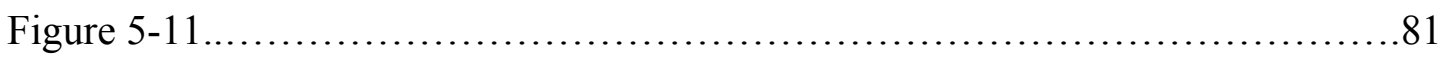

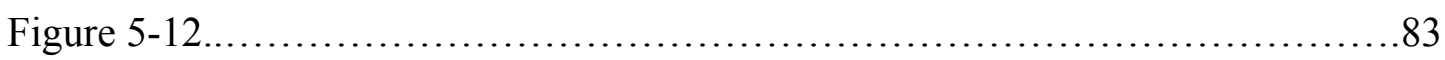

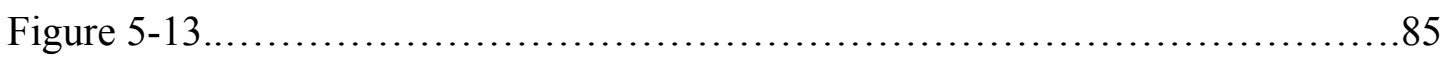

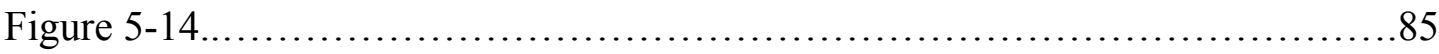

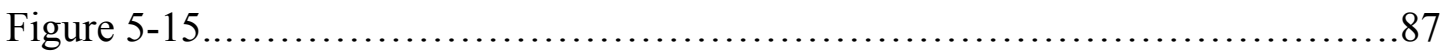

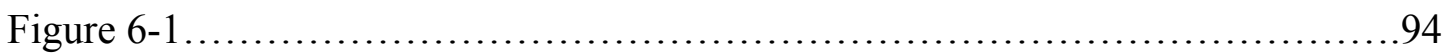

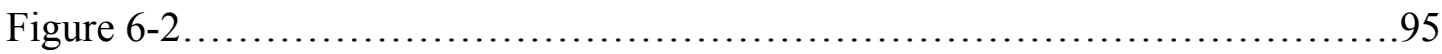

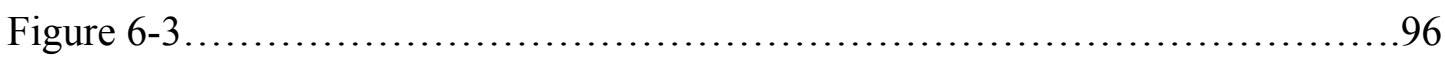

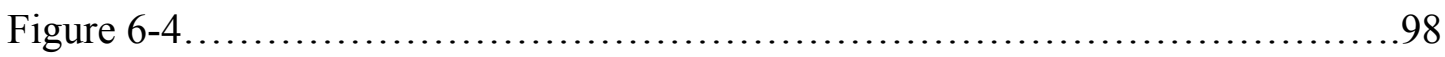

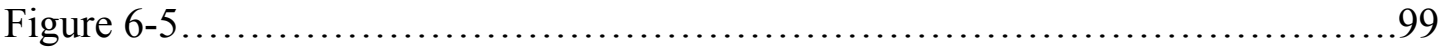

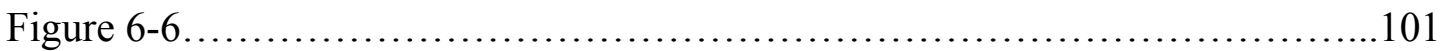

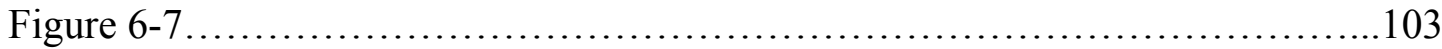

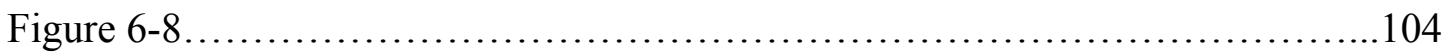

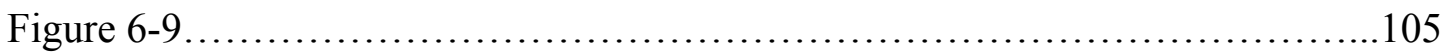

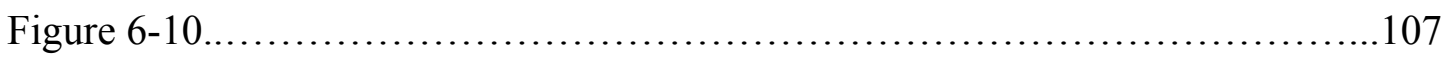

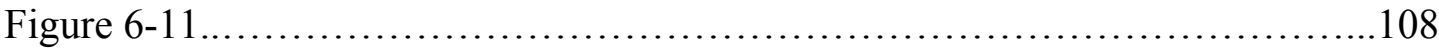

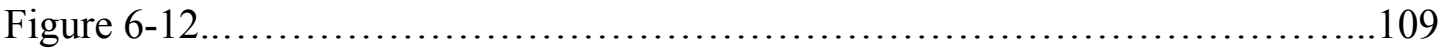

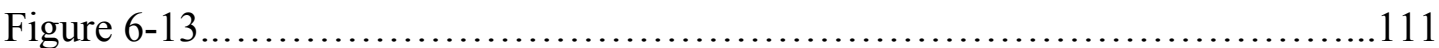

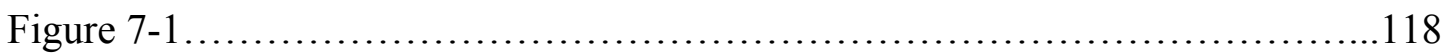

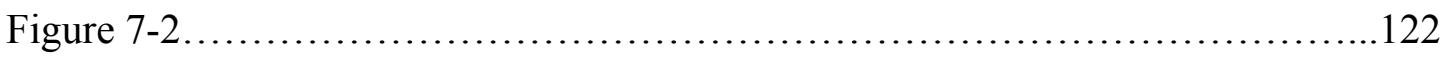

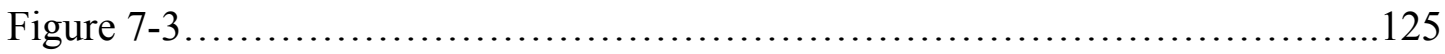

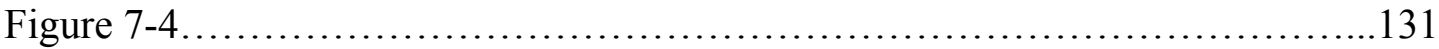

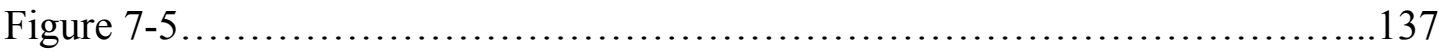

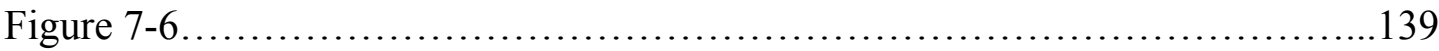

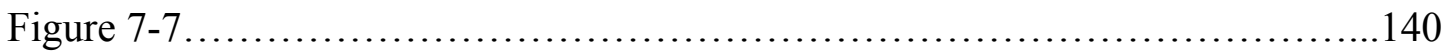

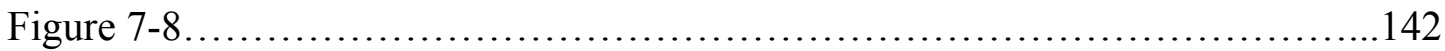

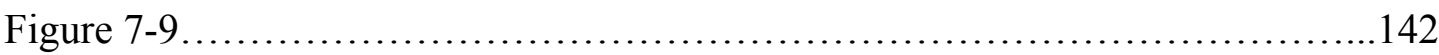

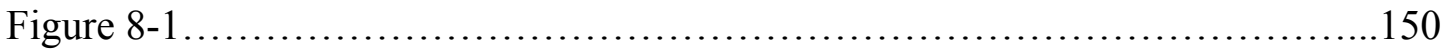

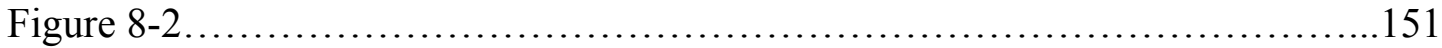




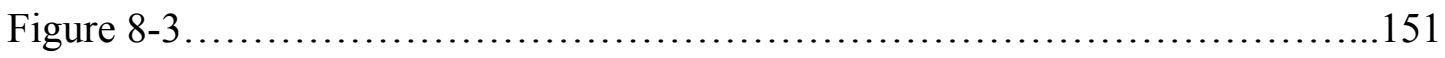

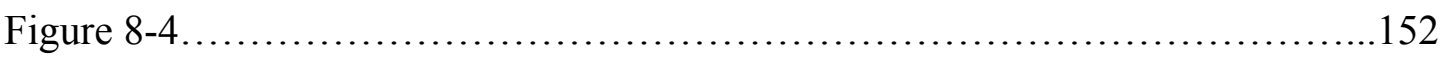

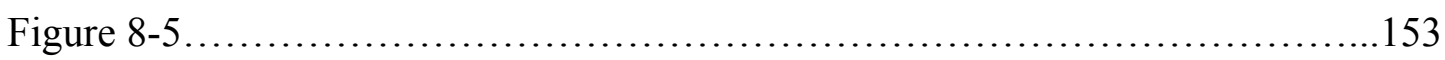

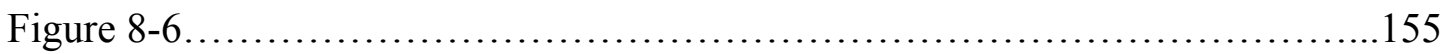

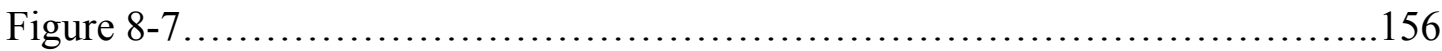

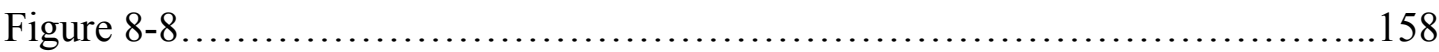

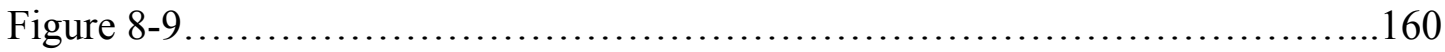

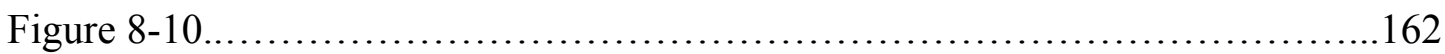

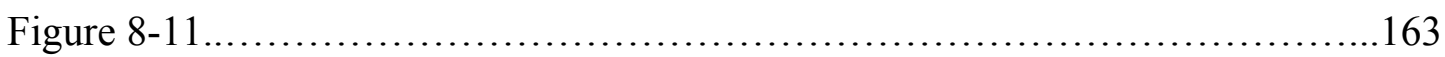

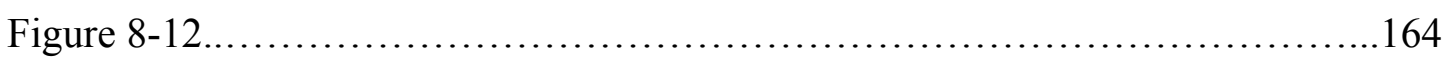

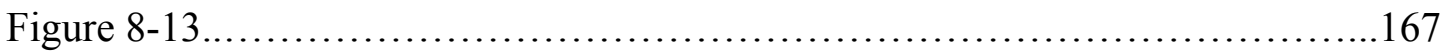

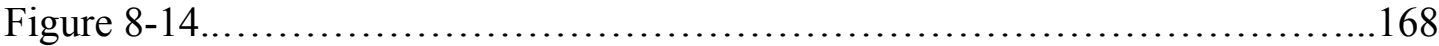

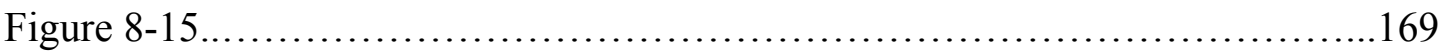

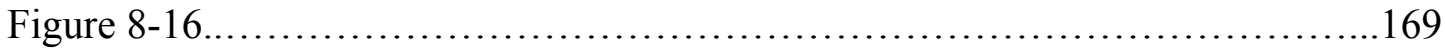

\section{B. List of Tables}

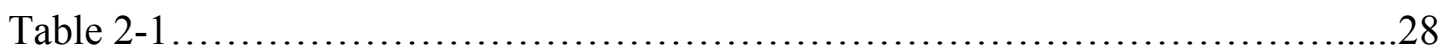

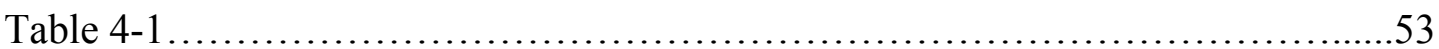

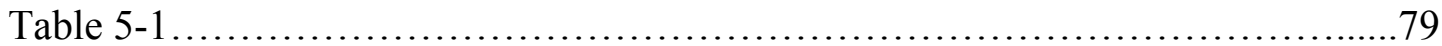

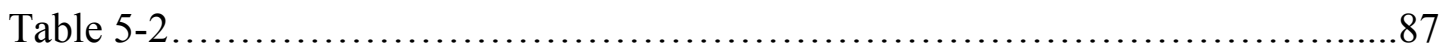

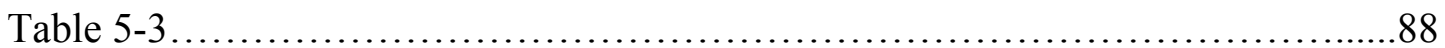

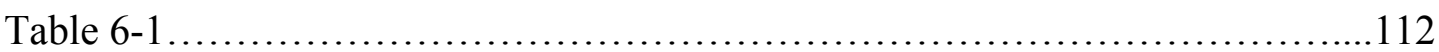

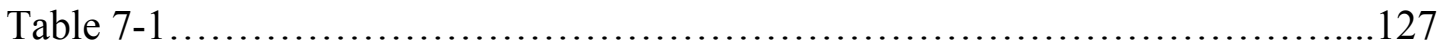




\section{Sample Calculations}

\section{Sample Calculations for Chapter 4.}

Below are sample calculations of the size scales presented in Table 1 based on polymer dry thickness of $6.40 \mathrm{~nm}$. The expressions are obtained from Kim et. al. ${ }^{237}$ and numbers in parenthesis represent the equation numbers as appeared in their paper.

\section{Definition of parameters.}

In their paper,

$h$ is defined as brush height in a fully-stretched conformation.

Since spot drying involves the removal of macroscopic bulk water, the system technically undergoes a transition from a fully wet to fully dry state. Here, our approximations are based on the case where protein-brush interactions are assumed to occur just when the top of the brush is starting to dry up (i.e. we assume the evaporation of bulk water to be vertically homogeneous). At this point, based on the water-barrier model, the underlying layer is effectively still water-laden (i.e. brush is almost fully-stretched). We therefore take this into account and approximate $h=1.5 \mathrm{x}$ dry thickness obtained via ellipsometry. The correction factor 1.5 is based on the report that pristine POEGMA thickness can increase by $\sim 50 \%$ due to swelling in MilliQ water. ${ }^{24}$ Therefore for thickness of $6.40 \mathrm{~nm}$ :

$$
h \cong 1.5 \times 6.40=9.60 \mathrm{~nm}
$$

$a$ is defined as monomer size.

This was calculated from bulk density of the polymer $\left(1.105 \mathrm{~g} \mathrm{~cm}^{-3}\right.$ as stated by manufacturer).

$$
a=\left(\frac{360 \times 10000000^{3}}{1.105 \times 6.022 \times 10^{23}}\right)^{\frac{1}{3}}=0.8148 \mathrm{~nm}
$$

$\sigma$ is defined as the graft density. 
Since the initiator is deposited undiluted with other non-ATRP active moieties, we hereby assume the brushes formed were prepared at maximal grafting density. Hence, this was calculated based on maximum area, $a^{2}$, which can be occupied by the monomer. However, the intitiation step in surface-intiated ATRP is not $100 \%$ efficient. Based on Sumerlin, B. S., D. Neugebauer, et al. (2005). "Initiation Efficiency in the Synthesis of Molecular Brushes by Grafting from via Atom Transfer Radical Polymerization." Macromolecules 38(3): 702-708, we will consider $\sim 40 \%$ efficiency for our system. Therefore we include a correction factor of 0.4 in our calculation of $\sigma$.

$$
\therefore \sigma \cong 0.4 \times \frac{1}{a^{2}}=0.4 \times \frac{1}{0.8148^{2}} \cong 0.6025 \text { chains } / \mathrm{nm}^{2}
$$

$N$ is defined as the monomer units per polymer.

$$
\begin{gathered}
h=\sigma N a^{3} \\
\therefore N=\frac{h}{\sigma a^{3}} \cong \frac{9.60}{0.6025 \times 0.8148^{3}}=29.455
\end{gathered}
$$

Calculation of $\bar{\xi}_{\text {blob }}$ according to equation (7).

$$
\begin{gathered}
\bar{\xi}_{\text {blob }} \cong \frac{8 N a^{2}}{h} \cong \frac{1}{\sigma a} \\
\therefore \bar{\xi}_{\text {blob }} \cong \frac{1}{0.6025 \times 0.8148}=2.037 \mathrm{~nm}
\end{gathered}
$$

Calculation of $\xi_{\text {surf }}$ according to equation (8).

$$
\begin{gathered}
\xi_{\text {surf }} \cong\left(\frac{8 N a}{3 \pi^{2} \sigma}\right)^{\frac{1}{3}} \\
\therefore \xi_{\text {surf }} \cong\left(\frac{8 \times 29.455 \times 0.8148}{3 \pi^{2} \times 0.6025}\right)^{1 / 3}=2.208 \mathrm{~nm}
\end{gathered}
$$

Calculation of $b^{*}$ according to equation (18).

$$
b^{*}=\frac{2^{\frac{1}{3}}}{\pi} \times a\left(\frac{N a}{h}\right)^{\frac{2}{3}}=\frac{2^{\frac{1}{3}}}{\pi} \times 0.8148\left(\frac{29.455 \times 0.8148}{9.60}\right)^{\frac{2}{3}}=0.601 \mathrm{~nm}
$$


Calculation of $b_{\max }$ according to equation (21).

$$
b_{\max }=\left(\frac{N a^{2}}{2 \pi^{3} \sigma}\right)^{\frac{1}{4}} \sim N^{\frac{1}{4}}=N^{\frac{1}{4}}=2.330 \mathrm{~nm}
$$

\section{Sample calculations for Chapter 6.}

The graft density of pOEGMA was calculated based on the following equation:

$$
\sigma=\frac{h \rho N_{A}}{\bar{M}_{n}}
$$

Where $\sigma$ is the graft density (chains $/ \mathrm{nm}^{2}$ ), $h$ is the layer thickness determined by ellipsometry, $\rho$ is the bulk density of the polymer $\left(1.105 \mathrm{~g} \mathrm{~cm}^{-3}\right.$ was used as stated by manufacturer), $N_{A}$ is Avogadro's number and $\bar{M}_{n}$ is the number-average molecular weight of the polymer chains on the surface.

$\bar{M}_{n}$ was determined by conversion (taking ideal case of $100 \%$ ) $\mathrm{x}$ targeted degree of polymerization (ratio of $[\mathrm{OEGMA}] /[$ bromo-initiator] $=100) \times$ MW of OEGMA (360 $\mathrm{g} / \mathrm{mol})$

$$
\begin{gathered}
\bar{M}_{n}=1 \times 100 \times 360=36000 \\
\therefore \sigma=\frac{18.92 \times 1.105 \times 6.022 \times 10^{23}}{36000} \times \frac{1 \mathrm{~nm}{ }^{3}}{10000000 \mathrm{~cm}}=0.35 \text { chains } / \mathrm{nm}^{2}
\end{gathered}
$$

The surfacial blob size is calculated by combining equations (7) and (8) from Kim et. al. $^{237}$ :

$$
\xi_{\text {surf }}=\frac{8 h}{3 \pi^{2} a^{2} \sigma^{2}}
$$


$a$ is defined as monomer size and was calculated from density:

$$
\begin{gathered}
a=\frac{360 \times 10000000^{3}}{1.105 \times 6.022 \times 10^{23}}=0.5410 \\
\therefore \xi_{\text {surf }}=\left(\frac{8 \times 18.92}{3 \pi^{2} \times 0.5410^{2} \times 0.35^{2}}\right)^{1 / 3}=5.22 \mathrm{~nm}
\end{gathered}
$$

